\title{
HECT-type Ubiquitin Ligases in Nerve Cell Development
}

\author{
Dissertation \\ in partial fulfillment of the requirements \\ for the award of the degree \\ "Doctor rerum naturalium" \\ of the Georg August University Göttingen, \\ Faculty of Biology
}

within the doctoral program

Neurosciences

of the Georg August University School of Science

submitted by

Mateusz Cyryl Ambrożkiewicz

Born in Puławy, Poland

Göttingen, 2015 


\section{Thesis Committee}

Prof. Nils Brose, Department of Molecular Neurobiology, Max Planck Institute for Experimental Medicine, Göttingen

Dr. Judith Stegmüller, Cellular and Molecular Neurobiology, Max Planck Institute for Experimental Medicine, Göttingen

Prof. Ahmed Mansouri, Research Group Molecular Cell Differentiation, Max Planck Institute for Biophysical Chemistry, Göttingen

\section{Members of the Examination Board}

Referee: Prof. Nils Brose, Department of Molecular Neurobiology, Max Planck Institute for Experimental Medicine, Göttingen

$2^{\text {nd }}$ Referee: Dr. Judith Stegmüller, Cellular and Molecular Neurobiology, Max Planck Institute for Experimental Medicine, Göttingen

$3^{\text {rd }}$ referee: Prof. Ahmed Mansouri, Research Group Molecular Cell Differentiation, Max Planck Institute for Biophysical Chemistry, Göttingen

\section{Further members of the Examination Board}

Camin Dean, Ph. D., Trans-Synaptic Signaling, European Neuroscience Institute, Göttingen

Prof. Thomas Dresbach, Department of Anatomy and Embryology, University Medical Center Göttingen

Prof. Tobias Moser, Institute for Auditory Neuroscience and InnerEarLab, University Medical Center Göttingen

Date of oral examination: $19^{\text {th }}$ November 2015 


\section{DECLARATION}

Herewith I declare, that I prepared the Doctoral Thesis "HECT-type Ubiquitin Ligases in the Nerve Cell Development" on my own, and with no other sources and aids than quoted.

This work was partly supported by the Ph.D. program "Neurosciences" - International Max Planck Research School at the Georg August University Göttingen.

Mateusz Ambrożkiewicz

Göttingen, 21st September, 2015 
Niczego w życiu nie należy sie bać, należy to tylko zrozumieć.

Nothing in life is to be feared, it is only to be understood.

Maria Skłodowska-Curie (1867-1934)

You better work.

RuPaul Andre Charles (1960- )

Mojej siostrze i mamie.

For my sister and my mum. 


\section{TABLE OF CONTENTS}

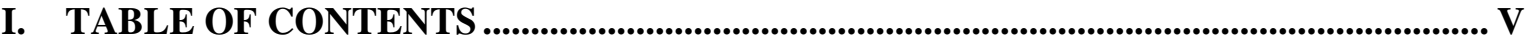

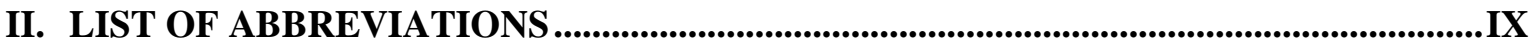

III. ABSTRACT ……......................................................................................................................... X

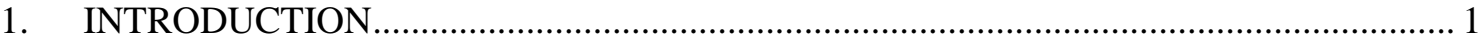

1.1. Development of cortical neurons in vivo ......................................................... 1

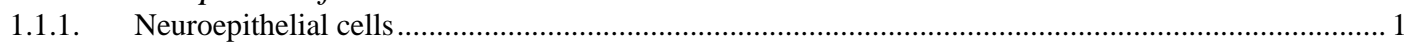

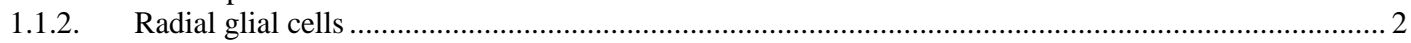

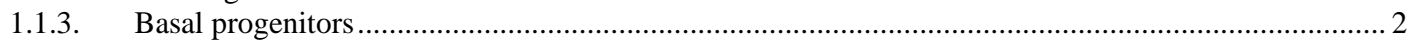

1.2. Neuronal migration in the developing brain .......................................................... 3

1.2.1. Initiation of radial migration and acquisition of cell polarity in vivo ............................................... 3

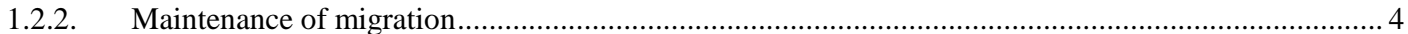

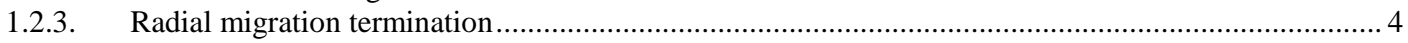

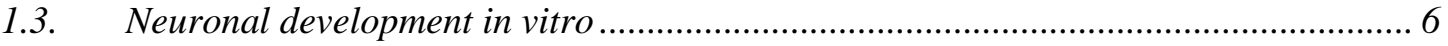

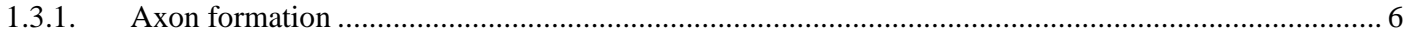

1.4. Dendritic spines and structural basis of synaptic transmission ……............................. 7

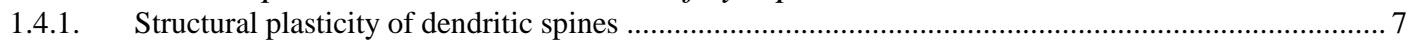

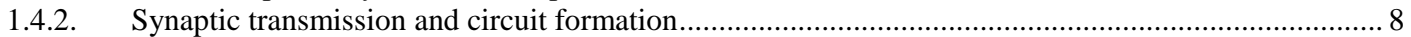

1.5. In utero electroporation in studies of neuronal development …...................................... 8

1.6. Posttranslational control of gene expression: biology of ubiquitylation .................... 10

1.6.1. Nedd4 family ubiquitin ligases of HECT type....................................................................... 11

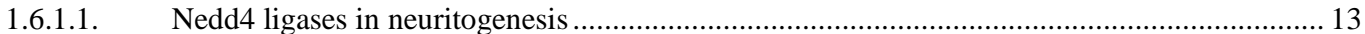

1.6.1.2. The role of Nedd4 ligases in regulation of neurotransmitter receptors, ion channels and transporters 13

1.6.2. Molecular basis of Angelman syndrome and the role of Ube $3 a$ in developing and mature neurons .. 15

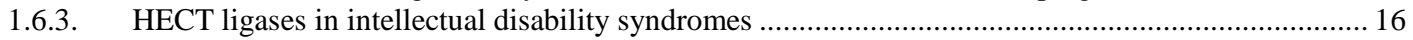

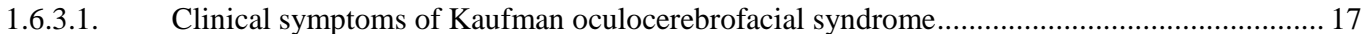

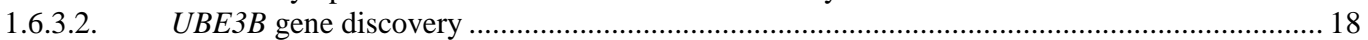

1.6.3.3. Mutations in UBE3B identified in KOS ........................................................................ 18

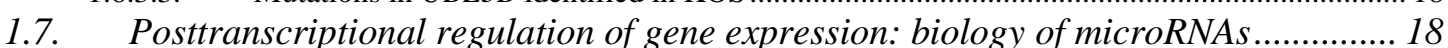

1.7.1. miRNA gene transcription and initial processing of miRNA precursors......................................... 19

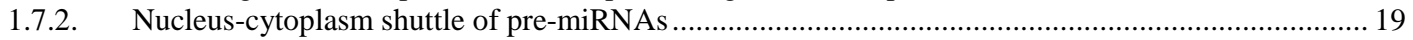

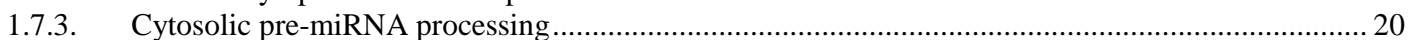

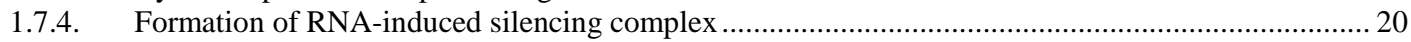

1.7.5. Strand selection and target mRNA recognition .......................................................... 20

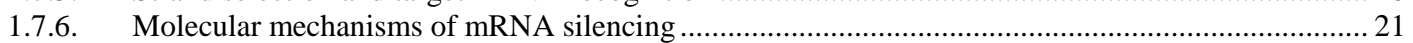

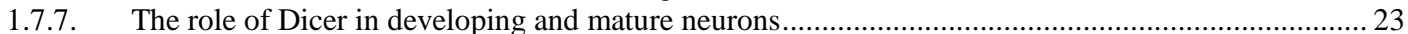

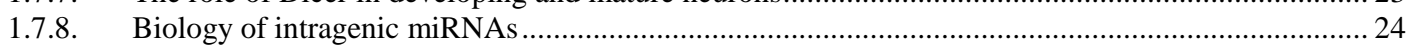

1.7.9. Interplay of Wwp2 ubiquitin ligase and intragenic miR-140 in control of gene expression.............. 24

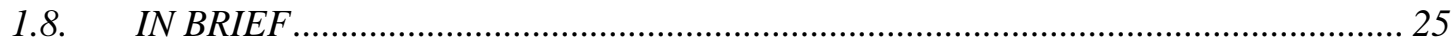

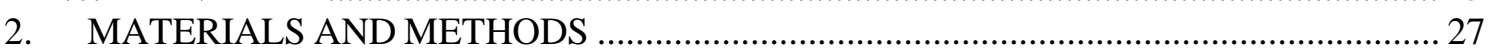

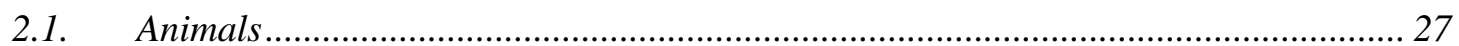

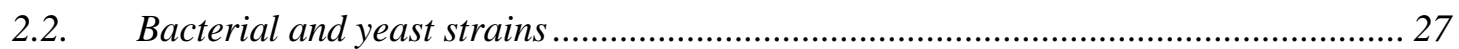

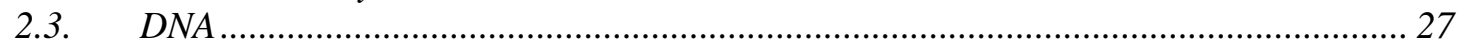

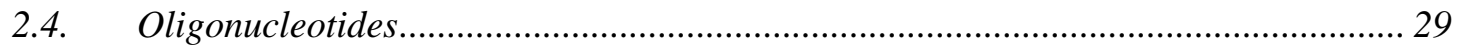

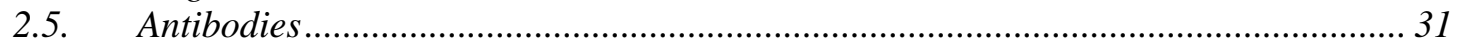

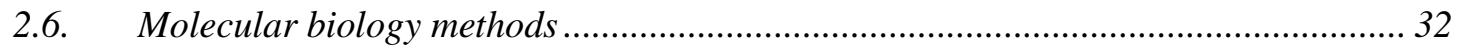

2.6.1. Electroporation of plasmid DNA into competent bacteria............................................................ 32

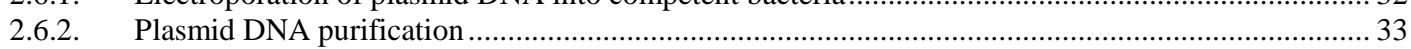

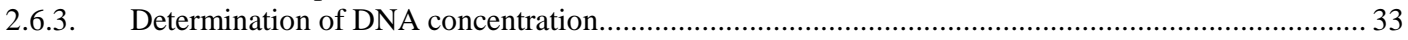

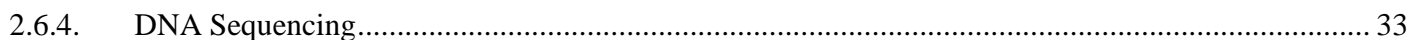

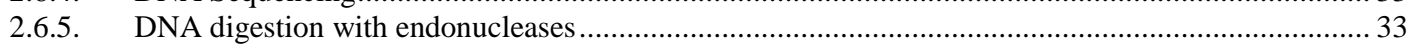

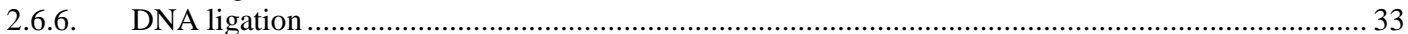

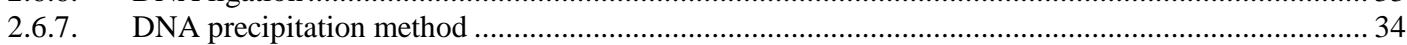

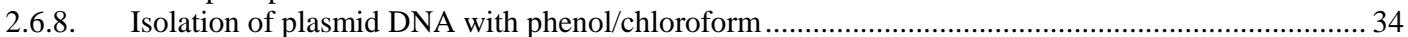

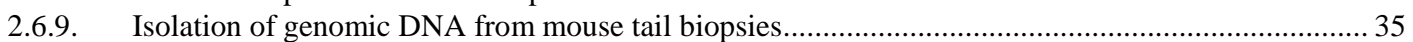

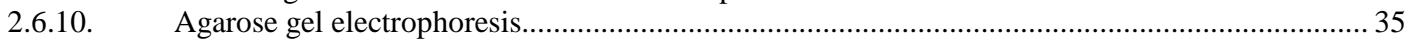

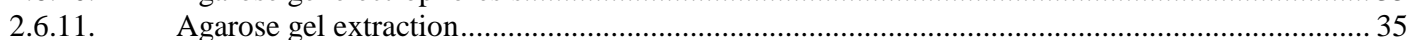

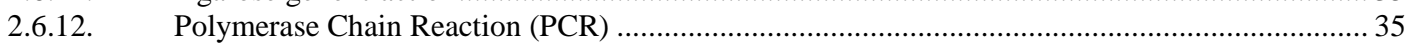

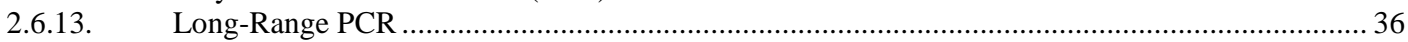




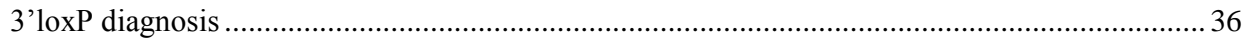

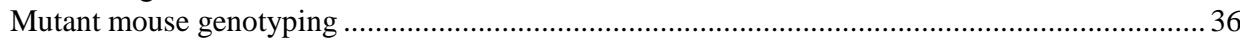

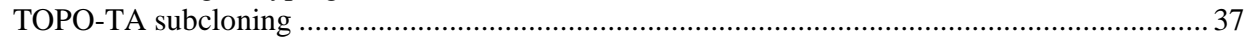

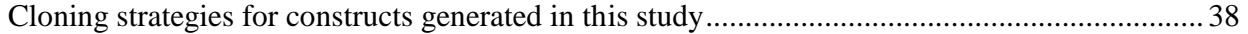

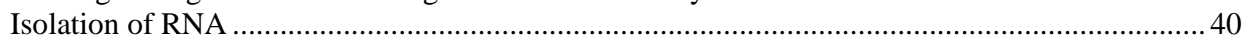

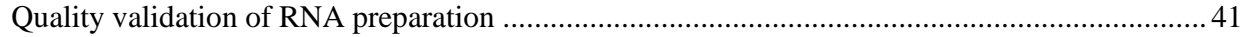

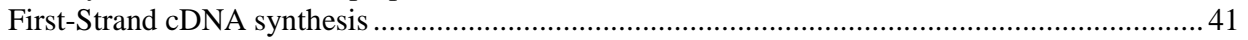

2.6.21. Real Time PCR (RT-PCR) for mRNA detection and quantification .......................................... 41

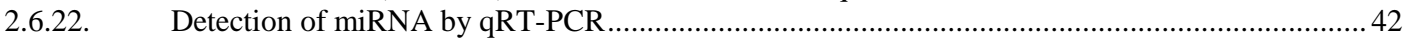

2.6.23. Isolation of the genomic DNA from Embryonic Stem Cells (ES Cells) ..................................... 43

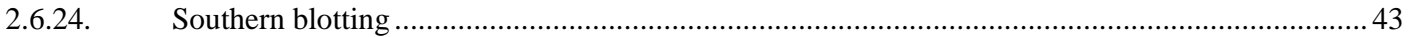

2.6.25. Yeast Two-Hybrid (Y2H) screening ……........................................................................ 44

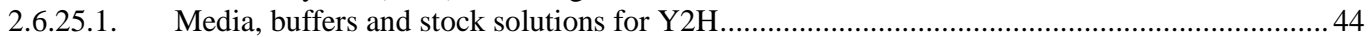

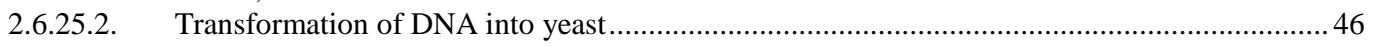

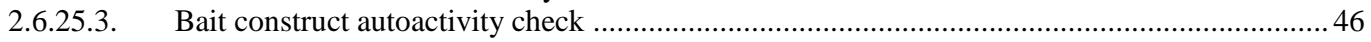

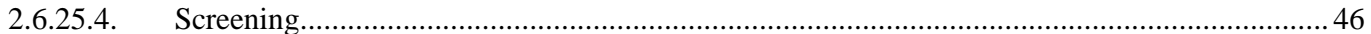

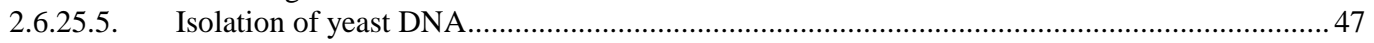

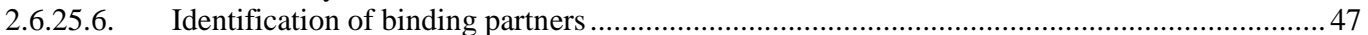

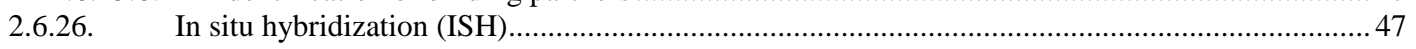

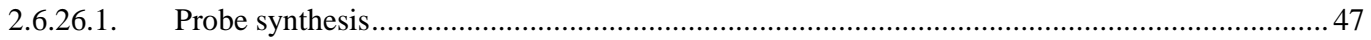

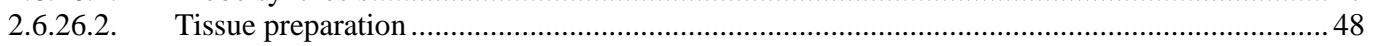

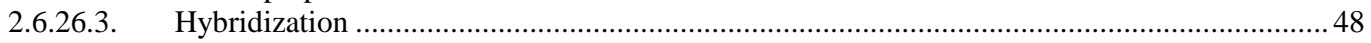

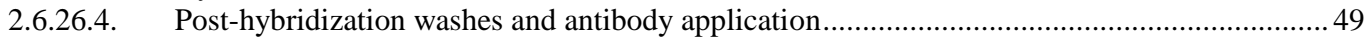

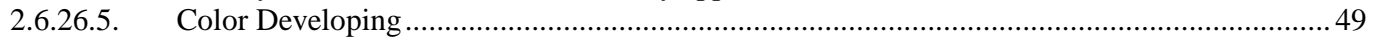

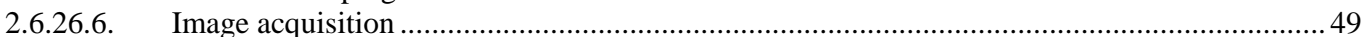

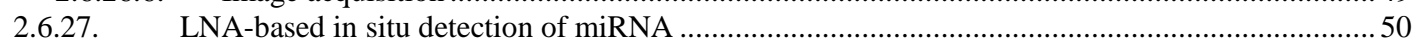

2.7. Biochemical experiments ......................................................................... 51

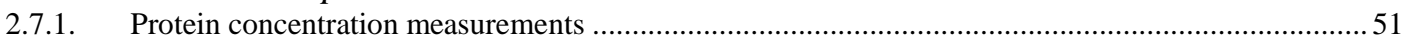

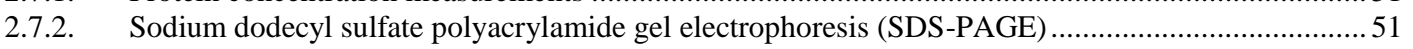

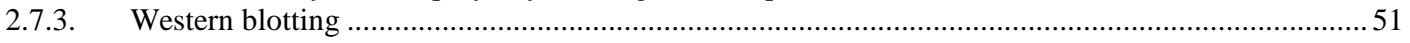

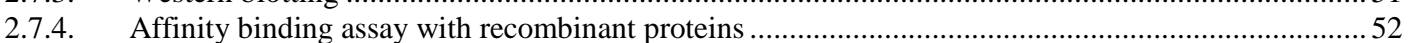

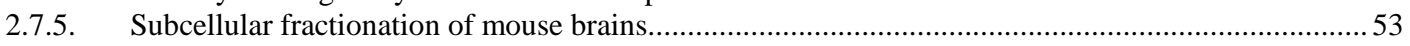

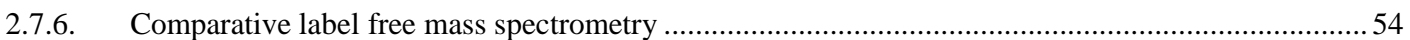

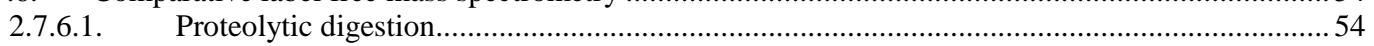

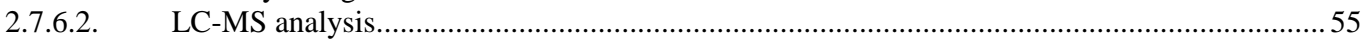

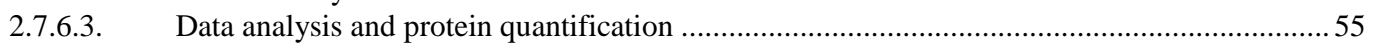

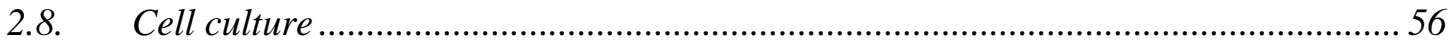

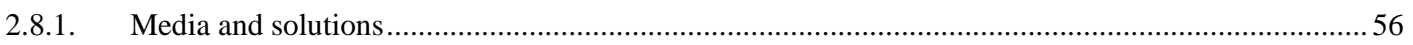

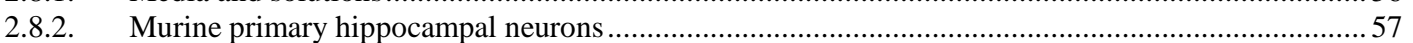

2.8.2.1. Treatment of coverslips for culturing primary neurons .......................................................5 57

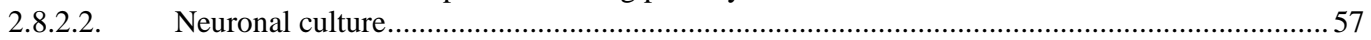

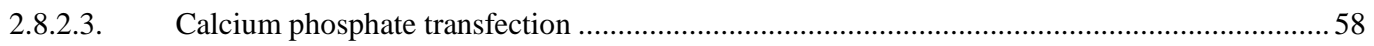

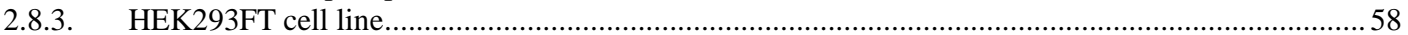

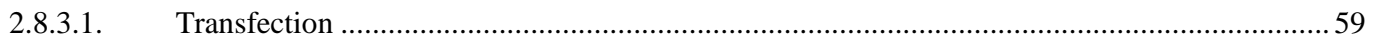

2.8.3.2. Lentivirus production and infection of primary hippocampal cells ......................................59

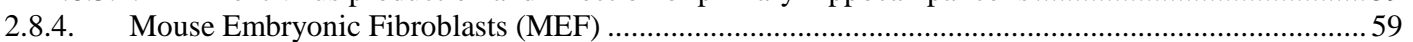

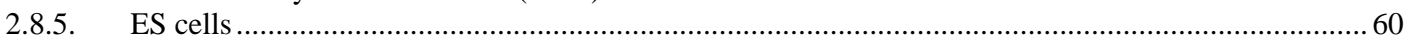

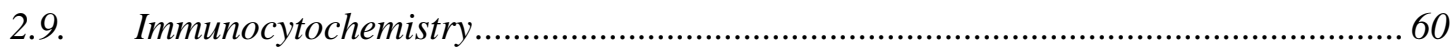

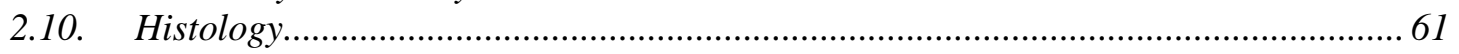

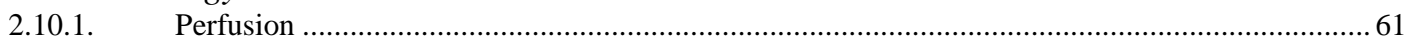

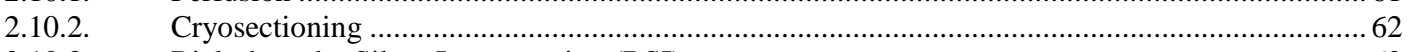

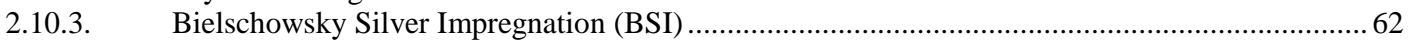

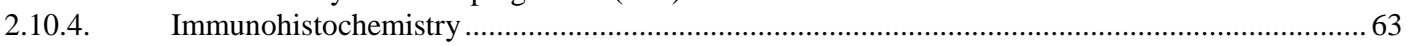

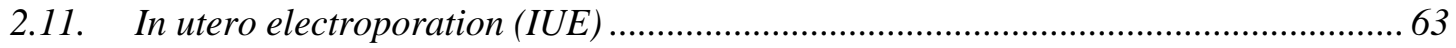

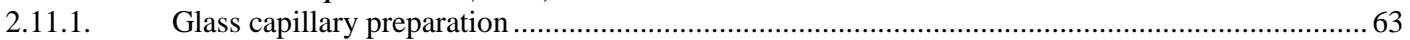

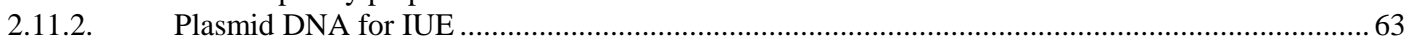

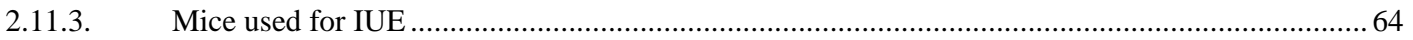

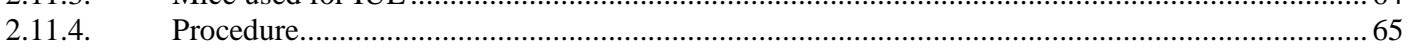

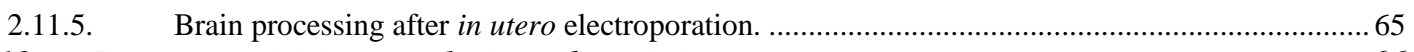

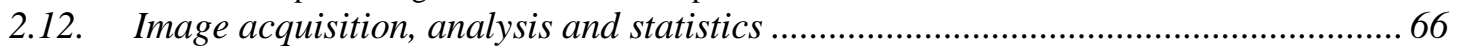

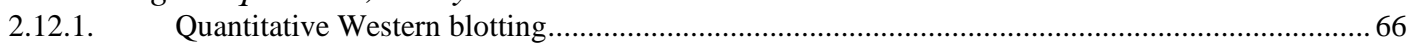

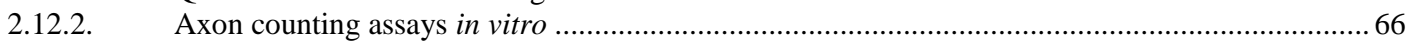

2.12.3. Quantification of distribution of cortical neurons and polarity classification ................................66

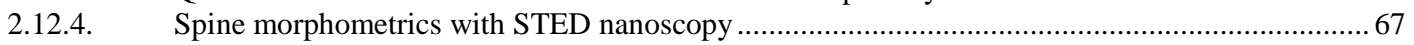

2.12.5. Quantification of axon length and dendritic tree complexity ................................................... 68

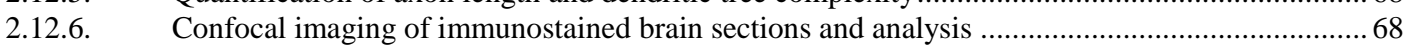




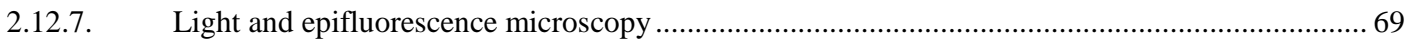

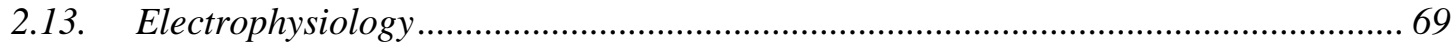

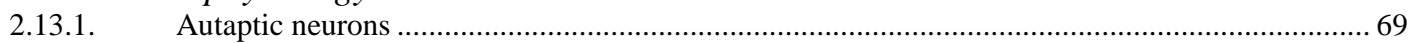

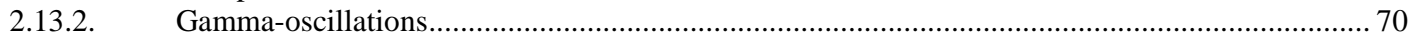

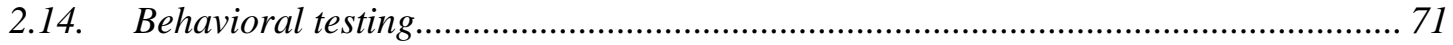

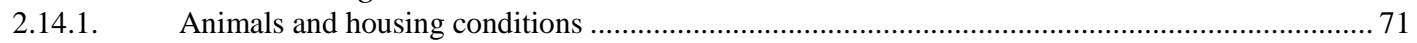

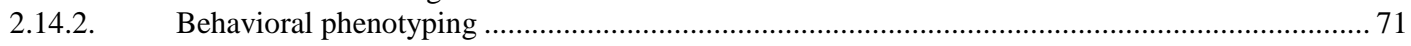

2.14.3. Spatial learning, and memory: Morris water-maze ............................................................... 71

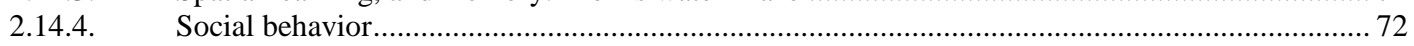

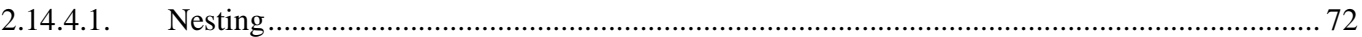

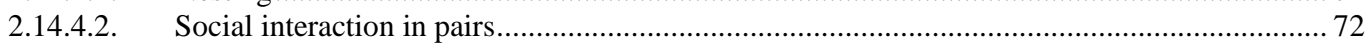

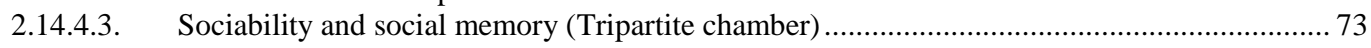

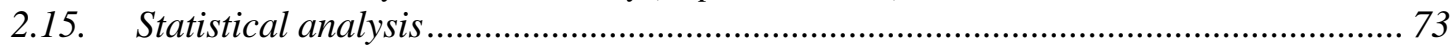

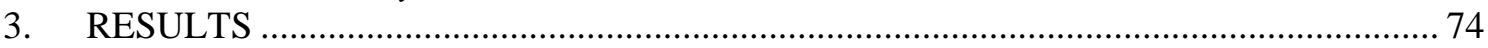

3.1. $m i R-140, W w p 1$ and $W w p 2$, and Sox 9 as regulators of neuronal development........ 74

3.1.1. Downregulation of Wwp1 and Wwp2 in primary hippocampal neurons leads to axon acquisition

defects 74

3.1.2. Knock-down of Wwp1 and Wwp2 in cortical progenitors leads to striking neuronal polarity defects

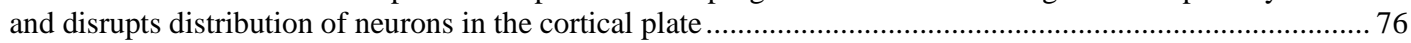

3.1.3. Genetic deletion of Wwp1 and Wwp2 leads to disturbances in axon acquisition in primary

hippocampal neurons and disrupts polarity of cortical pyramidal neurons in vivo ........................................... 81

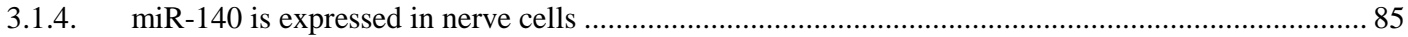

3.1.5. Loss of $m i R-140$ phenocopies the knockout of $W w p 1$ and $W w p 2$ in regard to axon-dendrite

specification in neurons and leads to aberrant distribution of developing nerve cells ..................................... 87

3.1.6. miR-140-3p, but not miR-140-5p is the mediator of axon specification in primary hippocampal neurons 91

3.1.7. Knock-down of miR-140-3p in cortical progenitors leads to persistent polarity aberrances and

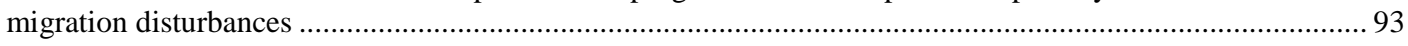

3.1.8. Altered distribution of neurons upon miR-140 depletion is not caused by aberrances in neurogenesis 96

3.1.9. Overexpression of miR-140 in primary hippocampal neurons abrogates axon formation................. 99

3.1.10. Detrimental effects of $m i R-140$ and Wwpl/Wwp2 loss in neurons do not seem to depend on

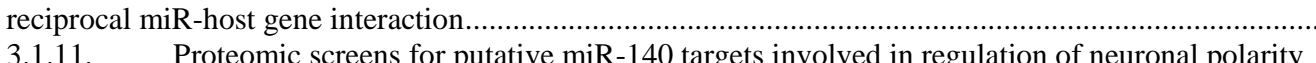

acquisition 102

3.1.12. Sox 9 is expressed in postmitotic neurons of the murine central nervous system ...................... 105

3.1.13. Sox 9 regulates axon specification in postmitotic primary hippocampal neurons ........................... 106

3.1.14. Sox 9 emerges as a potent regulator of neuronal distribution an a regulator of cortical neuron

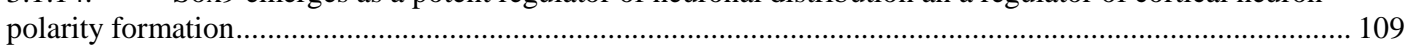

3.2. HECT ligases of Nedd4 family in mature neurons .............................................. 113

3.2.1. Wwp ligases control development of dendritic spines........................................................... 113

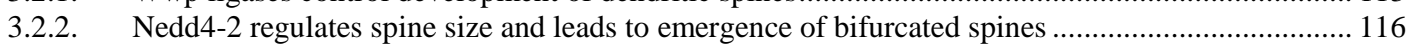

3.3. HECT type ubiquitin ligase Ube3b is essential for normal neuronal development and

function

3.3.1. Loss of Ube3b in mice recapitulates human Kaufman oculocerebrofacial syndrome ............................ 118

3.3.2. Ube $3 \mathrm{~b}$ is expressed in developing and adult murine central nervous system and is enriched at PSDs 121

3.3.3. Ube3b knockout abrogates neurite branching in primary hippocampal neurons ............................ 124

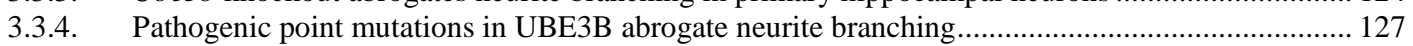

3.3.5. Neuron and glia specific Ube $3 b$ knockout mice display reduction of cortical thickness .................. 130

3.3.6. Increased spine density and longer spines with enlarged heads upon Ube 3 b deletion in hippocampal

neurons 133

3.3.7. Increased synaptic transmission in Ube $3 b$ KO neurons............................................................... 136

3.3.8. Imbalance of excitatory/inhibitory transmission in the hippocampus of $U b e 3 b \mathrm{cKO}$ mice.............. 139

3.3.9. Severe loss of spatial memory and disturbances in social interaction in Ube $3 b \mathrm{cKO}$ mice.............. 141

3.3.10. Proteomic screens reveal putative Ube3b substrates ............................................................ 145

4. DISCUSSION ............................................................................................ 149

4.1. Wwp ubiquitin ligases, miR-140, and Sox9 regulate neuronal polarity acquisition 149

4.1.1. Wwp1 and Wwp2 are indispensable for axon specification in primary hippocampal neurons ......... 149

4.1.2. Wwp1 and Wwp2 are essential for acquisition of neuronal polarity formation in vivo .................... 149

4.1.3. Nedd4 ligases orchestrate neuronal development .................................................................... 150

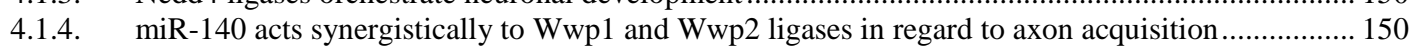

4.1.5. miR-140-3p acts as a biologically active guide strand to regulate neuronal polarity ...................... 151

4.1.6. Neuronal polarity formation is not controlled by reciprocal regulation of miR-140 and Wwp $1 / 2 \ldots 153$

4.1.7. miR-140 regulates mRNAs of proteins involved in polarity acquisition in neurons ........................ 153 
4.1.8. Sox9 regulates axon specification, polarity formation, and cortical distribution of postmitotic neurons 154

4.1.9. Sox9/Wwp1/2/miR-140-3p axis is critical for regulation of neuronal development....................... 156

4.2. HECT-type ubiquitin ligases and dendritic spines ........................................... 156

4.2.1. Wwp1 and Wwp2 ubiquitin ligases are potential negative regulators of synaptogenesis in cortical and

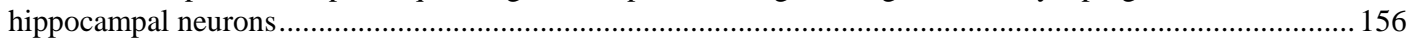

4.2.2. Nedd4-2 regulates dendritic spine morphology ........................................................................ 157

4.3. Ube3b, Kaufman oculocerebrofacial syndrome, and the molecular pathologies in intellectual disability

4.3.1. Neuron-specific loss of Ube3b in mice recapitulates neurological defects of Kaufman

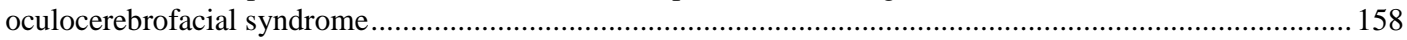

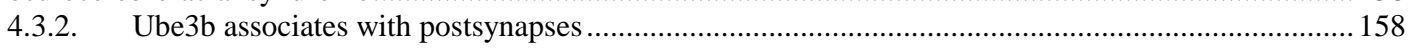

4.3.3. Ube3b is a positive regulator of neurite branching .............................................................. 159

4.3.4. Loss of Ube $3 b$ deficiency leads to increase in spine density, enlarged spine heads and longer spines

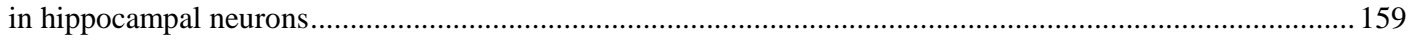

4.3.5. Neuronal morphology and intellectual disability ......................................................................... 160

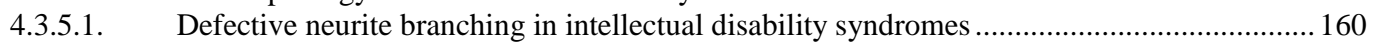

4.3.5.2. Dendritic spine abnormalities and mouse models of intellectual disability syndromes.......... 160

4.3.6. Deletion od $U b e 3 b$ leads to increased synaptic transmission and alters NMDAR to AMPAR ratio 161

4.3.7. Ube $3 b$ loss in neurons leads to imbalance between excitatory/inhibitory input to hippocampal circuits 162

4.3.8. Disrupted spatial memory and altered social interactions in Ube $3 b \mathrm{cKO}$ mice ............................. 163

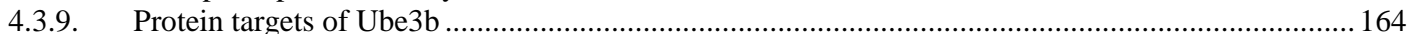

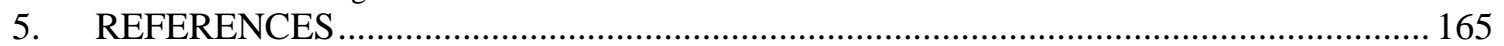

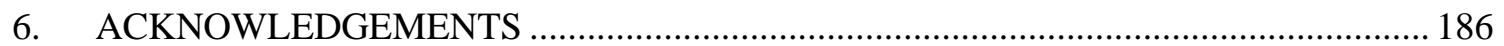

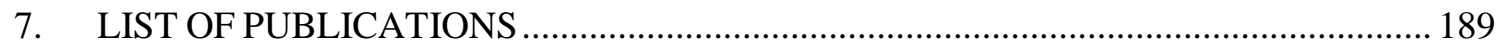

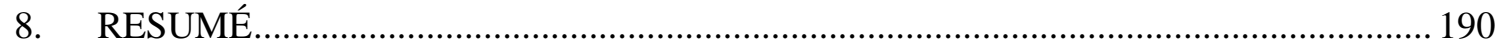




\section{LIST OF ABBREVIATIONS}

\begin{tabular}{|c|c|}
\hline AMPAR & $\alpha$-amino-3-hydroxy-5-methyl-4-isoxazolepropionic acid receptor \\
\hline ATP & Adenosine triphosphate \\
\hline C-terminal & Carboxy-terminal \\
\hline $\mathrm{cKO}$ & Conditional knockout \\
\hline DAPI & 2-(4-amidinophenyl)-1H-indole-6-carboxamidine \\
\hline $\mathrm{dcKO}$ & Double conditional knockout \\
\hline DIV & Day in vitro \\
\hline $\mathrm{dKD}$ & Double knock-down \\
\hline $\mathrm{dKO}$ & Double knockout \\
\hline $\mathrm{E} 1, \mathrm{E} 2, \mathrm{E} 3 \ldots$ & Embryonic day $1,2,3 \ldots$ \\
\hline EPSC & Excitatory postsynaptic current \\
\hline ES cell & Embryonic stem cell \\
\hline GABA & $\gamma$-aminobutyric acid \\
\hline GFP & Green fluorescent protein \\
\hline HA & Hemagglutinin \\
\hline HECT & Homologous to E6-AP C-terminus \\
\hline HRP & Horseradish peroxidase \\
\hline IP & Intermediate precursor \\
\hline KAR & Kainic acid receptor \\
\hline KD & Knock-down \\
\hline $\mathrm{KO}$ & Knockout \\
\hline LTP & Long term potentiation \\
\hline miRNA & microRNA \\
\hline N-terminal & Amino-terminal \\
\hline Nedd4 & Neural precursor cell expressed developmentally down-regulated 4 \\
\hline NMDAR & N-methyl-D-aspartate receptor \\
\hline $\mathrm{P} 1, \mathrm{P} 2, \mathrm{P} 3 \ldots$ & Postnatal day $1,2,3 \ldots$ \\
\hline PSD & Postsynaptic density \\
\hline Pvr & Vesicular release probability \\
\hline RGC & Radial glial cell \\
\hline RRP & Readily releasable pool \\
\hline $\mathrm{Ub}$ & Ubiquitin \\
\hline WB & Western blotting \\
\hline
\end{tabular}




\section{ABSTRACT}

Development of neurons begins in the middle of embryogenesis after closure of the neural tube. In the process of neurogenesis, neural stem cells generate nerve cells by asymmetric cell divisions at the ventricular zone of the brain. Immature neurons embark for their destination and start projecting axons and dendrites during migration. Growing axons are guided across the embryo towards their target neurons, and upon synaptogenesis and synapse elimination create complex architecture of the neural networks. Emerging evidence unveils that posttranslational modification by protein ubiquitylation and posttranscriptional regulation of gene expression by microRNAs are of particular importance for the nerve cell development. In this study, we demonstrate the roles of HECT-type ubiquitin ligases in newborn and mature nerve cells. Additionally, we characterize intronic microRNA and its host gene encoding for HECT-type ubiquitin ligase in developing nerve cells. Our work provides novel molecular insights for nerve cell development and contributes to present understanding of molecular cellular pathologies in intellectual disability syndrome. 


\section{INTRODUCTION}

Our emotions, memories ability to speak and hear all rely on uninterrupted neuronal connectivity [1,2]. Our brain is made up of 100 billion nerve cells, connected through synapses in circuits. Uncompromised functioning of neuronal networks depends on morphology of individual nerve cells $[3,4]$. Generation of mature neurons is a complex process that include molecular and cytological rearrangements controlled by multifarious cell signaling pathways.

\subsection{Development of cortical neurons in vivo}

Neural stem and progenitor cells give rise to neurons in the process of neurogenesis. Upon neurogenesis, newly born nerve cells migrate, differentiate, form axons and dendrites, and establish neuronal connectivity during synapse formation and elimination (Fig. 1A). Generation of astrocytes and oligodendrocytes (gliogenesis), myelination, angiogenesis, and blood-brain barrier formation accompany neuronal development in shaping the mature brain $[5]$.

\subsubsection{Neuroepithelial cells}

During development, neuroepithelial cells in the neural tube undergo a series of symmetrical proliferative divisions to then switch to asymmetrical mitoses, which result in self-renewal and generation of more lineage-restricted precursor cells (non-stem-cell progenitors termed basal progenitors), or differentiated neurons. Basal progenitors divide symmetrically, giving rise to two terminally differentiated postmitotic nerve cells [6-8].

The neural plate and neural tube is comprised of epithelial cells just before the onset of neurogenesis. Radial neuroepithelium spans the entire thickness of the neural tube. The nuclei of neuroepithelial cells perform characteristic alternate movement along the apicobasal axis, a phenomenon known as interkinetic nuclear migration. Since the cell cycle of neuroepithelial cells is not synchronized, the epithelium acquires a characteristic pseudostratified outlook. Interestingly, highly polarized neural stem cells present features typical for epithelial cells $[9,10]$. 


\subsubsection{Radial glial cells}

As development proceeds, the neuroepithelium lining the ventricular wall converts into a layered tissue with most of the neuronal progenitors. This region is collectively termed as ventricular zone. At E9-E10 in mice, the neurogenesis begins and neuroepithelial cells undergo several major cytological changes, which include downregulation of epithelial features and their differentiation status. Fundamentally, neuroepithelial cells undergo differentiative divisions giving rise to radial glial cells (RGCs), more fate-restricted progenitors. In contrast to neuroepithelial cells, murine RGCs are devoid of functional tight junctions at the apical end of the plasma membrane, but display adherens junctions associated with Zona Occludens-1 (ZO-1). [11-13]. Notably, progenitor cells induce the expression of astroglial markers, such as intermediate filament nestin [14].

RGCs maintain a characteristic bipolar shape of neuroepithelial cells with the long basal process reaching the pial surface, and shorter apical process, reaching out to the ventricular lumen [15]. Successively, RGCs replace neuroepithelial cells and directly or indirectly give rise to the majority of cortical neurons [16]. RGCs retain some features of neuroepithelial progenitors, such as apico-basal polarity of membrane protein distribution (e.g. apical expression of prominin-1, partitioning defective kinase-3, Par3/Par6/aPKC, atypical protein kinase C), contact with basal lamina, and interkinetic nuclear migration $[11,17,18]$. However, the nuclei of RGCs remain restricted to apical cytoplasm of the somata defining the ventricular zone. The last mitosis of RGCs takes place between E12 and E16 in mice, however, some of the apical progenitors preserve the radial glia characteristics until late postnatal stages and may function in adult neurogenesis [19].

Generation and maintenance of RGCs is dependent on numerous signaling cascades. Those include Notch/Delta-1, or neuregulin-1, Nrg-1, and ErbB2 tyrosine kinase receptor. Importantly, neurogenesis is controlled by transcription factors, such as sine-oculis related homeobox-3, Six3, empty spiracles homolog-2, Emx2, or paired box gene 6, Pax6 transcription factors [20-25].

\subsubsection{Basal progenitors}

Besides RGCs, another progenitor cells appear at the onset of neurogenesis, namely basal progenitors. These cells originate after mitoses of neuroepithelial cells and RGCs at the apical site of ventricular zone. During later stages of neurogenesis, basal progenitors define 
subventricular zone in the mammalian telencephalon [26,27]. Basal progenitors display yet another mode of cell division, paralleled by retraction of their apical process towards the ventricular surface. Notably, these cells specifically express non-coding RNA subventricular expressed transcript-1, Svet1, and transcription factors, such as eomesodermin homolog Eomes, also known as Tbr2, and cut-like homeobox-1 and -2, Cux1 and Cux2 [23,28,29]. Basal progenitors undergo symmetrical cell division, in which two daughter cells are generated, therefore subventricular progenitors function to increase the pool of neurons generated from ventricular precursors [30].

\subsection{Neuronal migration in the developing brain}

Newly born pyramidal neurons at ventricular zone embark for their future destination in the cortex in the process of radial migration, utilizing RGCs processes, that span the entire thickness of developing cortical plate. Tangential migration does not rely on interaction with RGCs and concerns GABAergic nerve cells, originating from the ganglionic eminence [31].

\subsubsection{Initiation of radial migration and acquisition of cell polarity in vivo}

Radially migrating neurons form specialized connections with RGC processes, known as junctional domains, critical for maintenance of the translocation towards pia [32]. The adhesion of migrating newborn neuron to the glial process specifies its leading process, facing towards the direction of translocation, and a trailing process, reaching out towards the ventricle. At this stage of development, newborn neurons display a characteristic bipolar morphology [33].

Importantly, newborn neurons inherit their axon-dendrite polarity from the apico-basal polarity of their progenitors. Consequently, the leading process acquires the dendritic fate, and the trailing one extends as an axon. Neurons specify an axon already during migration (E11-E18 in mice) [34]. 


\subsubsection{Maintenance of migration}

Interactions between molecules at the junctional domains, depend on the integrity of microtubules, which generate the force during the migratory movement. In line with this, significant migratory defects are observed upon mutations in genes critical for regulation of microtubule stability. X-linked lissencephaly in humans is caused by mutations in gene encoding for a protein doublecortin, Dcx. Dcx binds to microtubules and triggers their polymerization [35-37]. Another severe form of lissencephaly, Miller - Dieker syndrome, is caused by mutations in gene encoding another microtubule-associated protein, lissencephaly-1 protein, Lis1 (also known as platelet-activating factor acetylhydrolase isoform 1b, Pafah1b). Mutations in Lis1 result in slower migration of newborn neurons and disorganized cortical plate [38,39].

\subsubsection{Radial migration termination}

Radial movement of neurons abruptly terminates on the border between cortical plate and marginal zone, where nerve cells detach from RGCs and distribute within cortical layers. Early born neurons accumulate in deeper layers, and later-born nerve cells invade superficial cortical regions. Reeler mutant mouse shows inverted cortical layering, with early-born neurons distributing superficially in the cortex, and later-generated nerve cells in deeper layers [40]. The reeler locus encodes for a large protein, reelin secreted from Cajal-Retzius cells of the marginal zone, the outmost layer of developing cortex [41,42]. Cortical lamination is controlled by reelin, disabled-1, Dab1, apolipoprotein E receptor-2, ApoER2, and very low density lipoprotein receptor, VLDLR. ApoER2 and VLDLR bind reelin and this leads to phosphorylation of Dab1, a cytoplasmic adapter. Phosphorylated Dab-1 transduces an intracellular signal to induce rearrangements of actin cytoskeleton and modulate surface expression of integrins, which affects cellular adhesion. All four molecules represent a pathway that controls the termination of neuronal migration. [43-45]. 

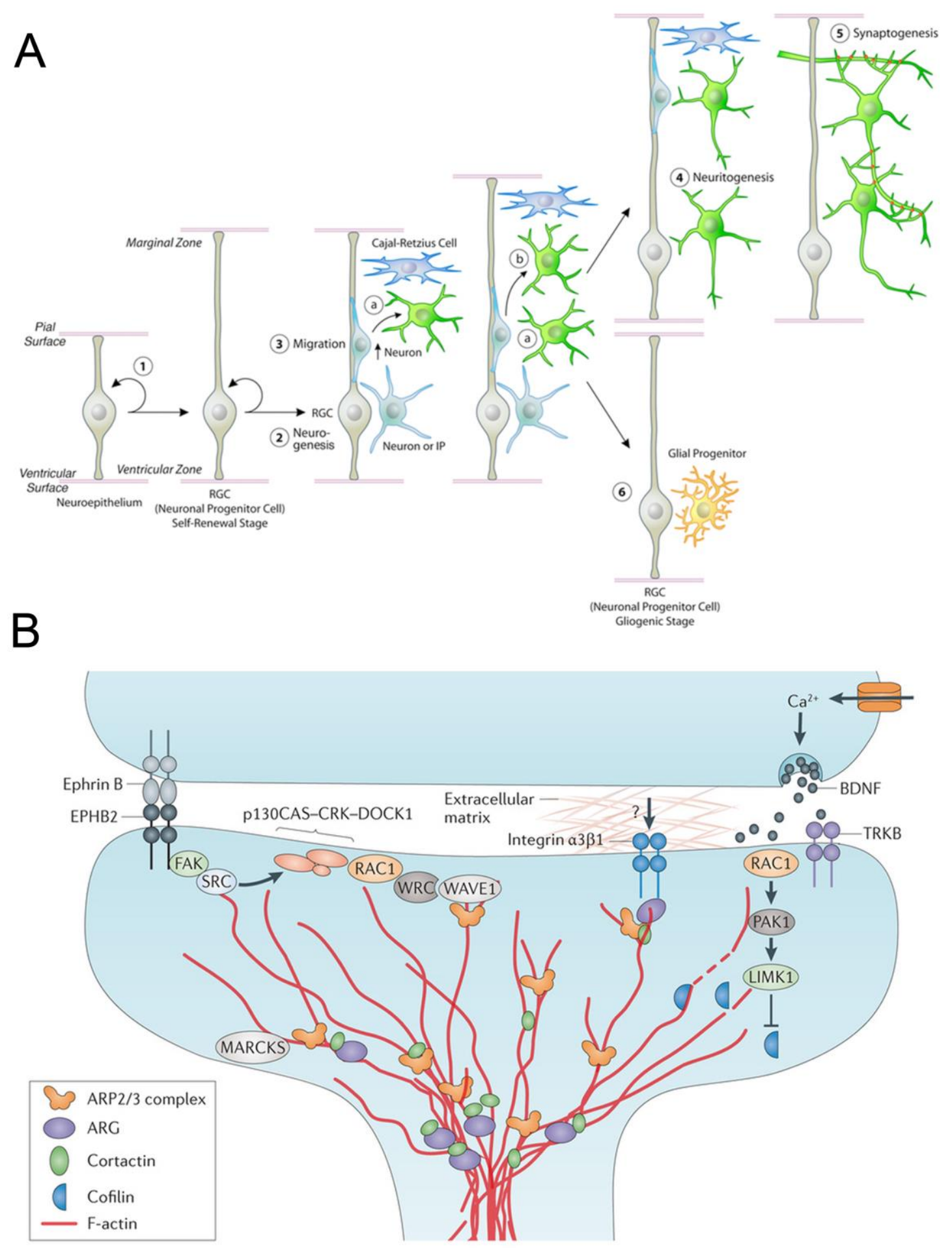

Fig. 1. Development of mammalian neurons. (A) Key steps of neuronal development in mammals. (1) After the closure of the neural tube neuroepithelial cells proliferate and give rise to radial glial cells (RGCs). (2) Symmetrical divisions of RGCs produce intermediate progenitors (IP). Asymmetrical divisions of RGCs give rise to immature neurons, which migrate along RGCs processes. (3) After receiving a stop signal from Cajal-Retzius cells, newborn neurons detach from RGCs (i), and distribute horizontally in an appropriate cortical layer to further differentiate. Neurons born later, migrate towards superficial cortical layers 
(ii). (4) Nerve cells establish their polarity by specification of neurites to dendrites and axons. (5) In the process of synaptogenesis and synapse elimination, axons establish contacts with their postsynaptic targets to organize nerve cells into circuits. (6) Soon after neurogenesis is complete, RGCs generate glia. (B) Postsynaptic spine and molecular signaling pathways for actin stabilization in the spine. Binding of Ephrin B to its receptor EphB2 recruits FAK and SRC kinases to activate p130 Crk associated substrate (p130CAS), adaptor molecule CRK, dedicator of cytokinesis protein 1 DOCK1 complex, and relay the activation signal to small GTPase, RAC1. RAC1 releases the WAVE regulatory complex (WRC) from WASP family verprolin-homologous protein-1, WAVE1, which in turn activates actin related protein $2 / 3$ (ARP2/3) to initialize new actin branch, and promote spine remodeling. Integrin $\alpha 3 \beta 1$ activates tyrosine kinase ARG (Abl2) leading to its association with cortactin, which activates ARP2/3 and stabilizes newly formed actin branch. Myristoilated alanine-rich C kinase substrate (MARCKS) mediates association of actin filaments with the membrane. Upon depolarization, brain derived growth factor, BDNF associates with its receptor TRKB and recruits RAC1 to promote $\mathrm{p} 21$-activated kinase 1, PAK1 and LIM-domain containing protein kinase, LIMK1 inhibition of cofilin which hampers its actin-destabilizing activity. (A) After [5] with permission of Nature Publishing Group (license number 3693560392918). (B) After [46] with permission of Nature Publishing Group (license number 3707551069487).

\subsection{Neuronal development in vitro}

Cultured primary hippocampal neurons progress through developmental stages that recapitulate those observed for postmitotic neurons in vivo. 12-24 hours after plating, neurons adhere to the substrate and display numerous lamellipodia and protruding filopodia (Stage 1), which follows with emergence of multiple neurites (Stage 2). Stage 3 represents a specification of neuronal polarity, during which one of the immature neurites extends rapidly as axon, while remainder neurites acquire dendritic fate. Next, neurites undergo rapid outgrowth (Stage 4) to establish synapses and functional circuits with other differentiated neurons characterized by dendritic spines and axon initial segment (Stage 5) [47].

\subsubsection{Axon formation}

Distribution of cellular organelles and cytoskeleton organization differ in dendrites and axon. Orientation of Golgi complex, mitochondria, and endosomes seem to correlate with the neurite acquiring axonal fate. In the developing hippocampal neurons, Golgi apparatus is deployed into an apical process that acquires dendritic identity [48].

Moreover, axons display a uniform microtubule organization, with the plus ends directed away from the soma. Dendritic microtubules display rather complex array of microtubule 
orientation. Arrangement of microtubules determines microtubule-dependent transport through kinesins and dyneins, characteristic for axons. Both type of neurites also present an intricate set of microtubule associated proteins, MAPs. MAP1B and Tau are specifically found in an axon, whereas MAP2a-c are dendritic proteins [49-52].

Moreover, disruption of actin polymerization leads to aberrant distribution of dendritic proteins into an axon. Actin cytoskeleton is critical for a polarized transport of organelles and proteins. Unique axonal F-actin organization appears critical for specification of axon initial segment and clustering of voltage gated sodium channels [53-55].

Not surprisingly, altering stability of cytoskeleton have dramatic consequences for the fate of the neurites. Taxol-induced stabilization of microtubules triggers neurons to project multiple axons. Moreover, local disruption of actin cytoskeleton at tips of the minor neurites turns them into axons $[56,57]$.

\subsection{Dendritic spines and structural basis of synaptic transmission}

Over the course of development, cortical neurons extend axons and dendrites to establish neural networks in synapse formation and elimination. Dendritic spines harbor majority of glutamatergic postsynapses in the mammalian brain. Morphology of individual spines distinguish several spine classes, i.e. stubby, filopodia, thin, mushroom, and bifurcated type. Dendritic spines are rich in F-actin that defines their shape and allows for activity-dependent shape remodeling. High-frequency synaptic stimulation promotes actin polymerization and consequently results in spine enlargement. Conversely, low-frequency stimulation leads to actin depolymerization and results in shrinkage of dendritic spines [58-60].

\subsubsection{Structural plasticity of dendritic spines}

Actin dynamics within the spine distinguishes a spine shell with fast actin turnover, and a spine core, characterized by more stable actin. Due to a rather stable actin core, spines keep their positions on the dendritic shafts, however, display dynamic morphological changes of the outer shell [46].

Actin binding proteins, altering stability of actin are therefore critical in maintaining structural plasticity of spines (Fig. 1B). Actin-related protein 2/3 complex (Arp2/3) nucleates new actin branches on existing actin filament, allowing for spine shape remodeling. Loss of Arp2/3 in neurons results in loss of glutamatergic synapses of hippocampal and cortical 
nerve cells, and reduction of synaptic transmission. On the whole, long term maintenance of dendritic spines relies of molecular machinery controlling actin stability in response to extracellular signals $[61,62]$.

\subsubsection{Synaptic transmission and circuit formation}

Majority of glutamatergic presynaptic inputs converge on postsynaptic dendritic spines. Basal neurotransmission in the central nervous system is mediated by AMPA-type and kainate glutamate receptors (AMPARs and KARs, respectively). Activation of NMDA receptors (NMDARs) elicits structural and functional plasticity, which underlies the basis of long-term potentiation (LTP). Scaffolding proteins, such as postsynaptic density-95, PSD95, or membrane associated guanylate kinase, WW, and PDZ containing 2, Magi-2 (also known as S-SCAM) anchor and cluster neurotransmitter receptors at the synapses. Deficiencies in postsynaptic scaffold follow with dramatic consequences for synaptic plasticity, resulting in compromised activity of neuronal circuits. That in turn may lead to disturbances of cognitive abilities and alteration of behavioral traits.

\subsection{In utero electroporation in studies of neuronal development}

In utero electroporation constitutes a powerful method to study of different aspects of neuronal development, from cell fate determination to spine morphology of nerve cells. DNA of interest is injected to the lateral ventricles of the embryo and electroporated to neuronal progenitors of the ventricular zone. In utero transfection of neocortical neuroepithelium, ammonic neuroepithelium, lateroventral pallial neuroepithelium, ganglionic eminence, or optic vesicle allows to study neurons of different brain regions they give rise to, such as cerebral cortex, hippocampus, piriform cortex and amygdala, interneurons or retina, respectively [19,63-71] (Fig. 2). 
A

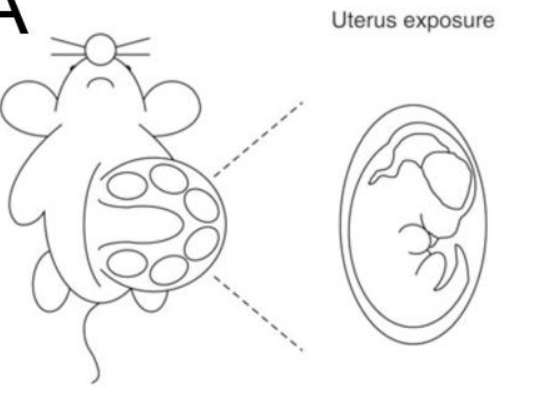

B
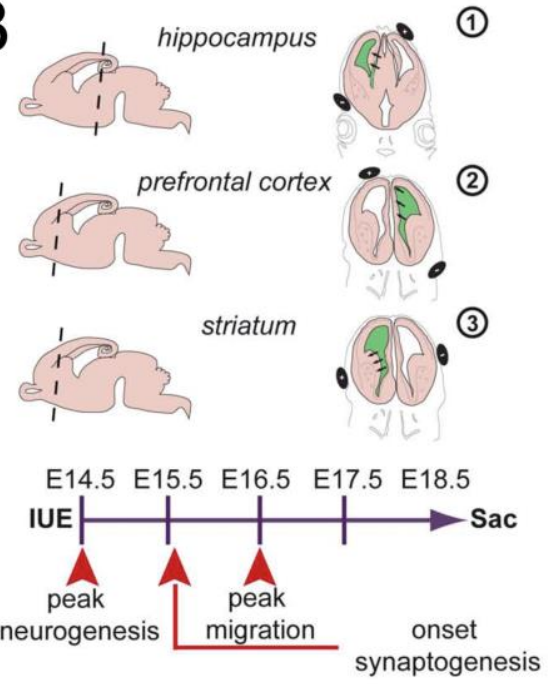
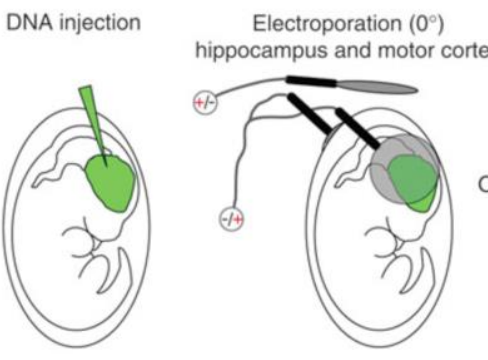

Electroporation $\left(90^{\circ}\right)$ visual cortex OR

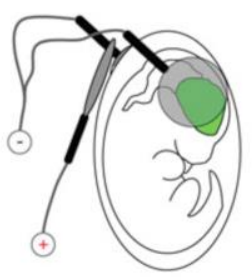

Fig. 2. In utero electroporation (IUE). (A) The procedure of IUE. Under deep anesthesia, the uterus of a pregnant mouse is exposed, DNA is injected into the lateral ventricles of the embryonic brains and electroporated to neuronal progenitors by application of electric current. (B) Depending on the age of the embryo and positioning of the electrodes depicted on the scheme, precursors of different brain regions are transfected. (C) Overview of DAPI staining and Venus fluorescence in P21 coronal brain section after in utero electroporation at E14.5. Neuronal precursors in cortical and ammonic neuroepithelium were transfected with plasmid encoding Venus. Note transfected neurons in the cerebral cortex, cingulate cortex, and CA1 - CA3 fields of hippocampus. Scale bar, $1 \mathrm{~mm}$.

(A) After [72] with permission of Nature Publishing Group (under a Creative Commons license, Attribution Noncommercial). (B) After [73] with permissions of Frontiers Media SA.

Maturation of neurons, from newly born RGC daughter cells to a nerve cell harboring dendritic spines, structurally and functionally integrated into neuronal networks is precisely controlled at each step through numerous signaling cascades. Effector proteins responsible for certain aspects of neuronal development are regulated on the level of their transcription, or posttranslationally. 


\subsection{Posttranslational control of gene expression: biology of ubiquitylation}

Posttranslational modifications of proteins by ubiquitylation involves conjugation of $10 \mathrm{kDa}$ globular ubiquitin (Ub) to a target proteins in cascade enzymatic reaction. First, E1 activating enzyme catalyzes conjugation of Ub with AMP through a high energy bond. UbAMP complex is covalently bound to the cysteine residue of E1. Ub activated in such a manner is subsequently shuttled onto E2 Ub conjugating enzyme and further onto the target protein with assistance of E3 ubiquitin ligase. Ultimately, protein ubiquitylation comprises of a formation of an isopeptide bond between $\varepsilon$-amino group of a lysine moiety in a substrate protein and the carboxyl rest of glycine at the C-terminus of Ub molecule (Fig. 3A). There is an evolutionary divergence considering the amount of genes encoding for enzymes mediating ubiquitylation reaction. One or two E1, about thirty E2, and around six hundred E3 are encoded in the human genome, but E3 ligases mediate specific protein-protein interaction and confer the specificity of ubiquitylation. Importantly, ubiquitylation can be reversed in a reaction catalyzed by proteases collectively named deubiquitinases (DUBs). Ub can be either cleaved off from its substrate, or ubiquitin chains can undergo enzymatic trimming [74-80].

Proteins can be coupled to a single Ub moiety, or can be decorated with Ub chains. Chain formation employs one or several lysine groups (Lys6, Lys11, Lys27, Lys29, Lys33, Lys48, and Lys63) of Ub. Ub-multimers can acquire forms of homotypic linkages to forked conformations. Importantly, the lysine utilized for chain formation specifies the functional consequence of conjugation of the Ub-chain to the substrate protein. Polyubiquitin chains comprised of Lysine-48 $\mathrm{Ub}$ moieties have been thought to serve as the main species of ubiquitin modification [81,82]. As discovered in the early 1980s, conjugation of Lys-48linked Ub chain destines substrate proteins for proteasomal degradation. Nonetheless, recent data illustrates that Lys-11 and Lys-63 alongside Lys-48 chains serve as the major ubiquitin modifications in the rat brain [83]. Functional consequences of Lys-63 polyubiquitylation include control over genomic stability [84] and regulation of transmembrane proteins [85,86], whereas Lys-11 linked poly-Ub chains control cell cycle progression [87] and are involved in endoplasmic reticulum-associated degradation pathway [88].

E3 ubiquitin ligases fall into two major families based on their biochemistry of ubiquitylation. Really Interesting New Gene (RING) ligases do not act as Ub acceptor themselves, but bring the $\mathrm{E} 2$ bound to $\mathrm{Ub}$ and the substrate protein in the molecular vicinity to facilitate $\mathrm{Ub}$ transfer. Homologous to E6-AP C-terminus (HECT)-type ligases covalently 
conjugate $\mathrm{Ub}$ moiety to a cysteine residue in their catalytic domain and thereupon transfer $\mathrm{Ub}$ onto their target proteins. While RING-type enzymes are the preponderant E3 ligases, only 28 of HECT-type have been identified to date [89,90].

The HECT domain is homologous between the members of HECT-type ligases and comprised of N-terminal, E2-enzyme-binding lobe, and C-terminal lobe, harboring a catalytic cysteine residue. Juxtapositioning of catalytic centers of E2 and E3 enzymes during $\mathrm{Ub}$ transfer is facilitated by flexible hinge region, bridging both lobes. [91]. Based on the domain organization and homology of N-termini, HECT-type ligases are further grouped into three subfamilies: Nedd4 family, HERC family, and other HECT-type ligases [92-94] (Fig. 3B).

\subsubsection{Nedd4 family ubiquitin ligases of HECT type}

Nedd4 family ligases represent a unique group among HECT-type ligases due to their distinct domain organization (Fig. 3C). To date nine members of Nedd4 ligases have been identified in the human genome and majority of them have been implicated in different aspects of neuronal development [92].

Nedd4 ligases share a C-terminus calcium binding C2 domain, several WW-domains, critical for substrate-ligase interactions, and C-terminus catalytic HECT domain. Homologous domain organization implicates a uniform activation of Nedd4 ligases, e.g. by calcium, or through phosphorylation by up-stream activators [95]. 


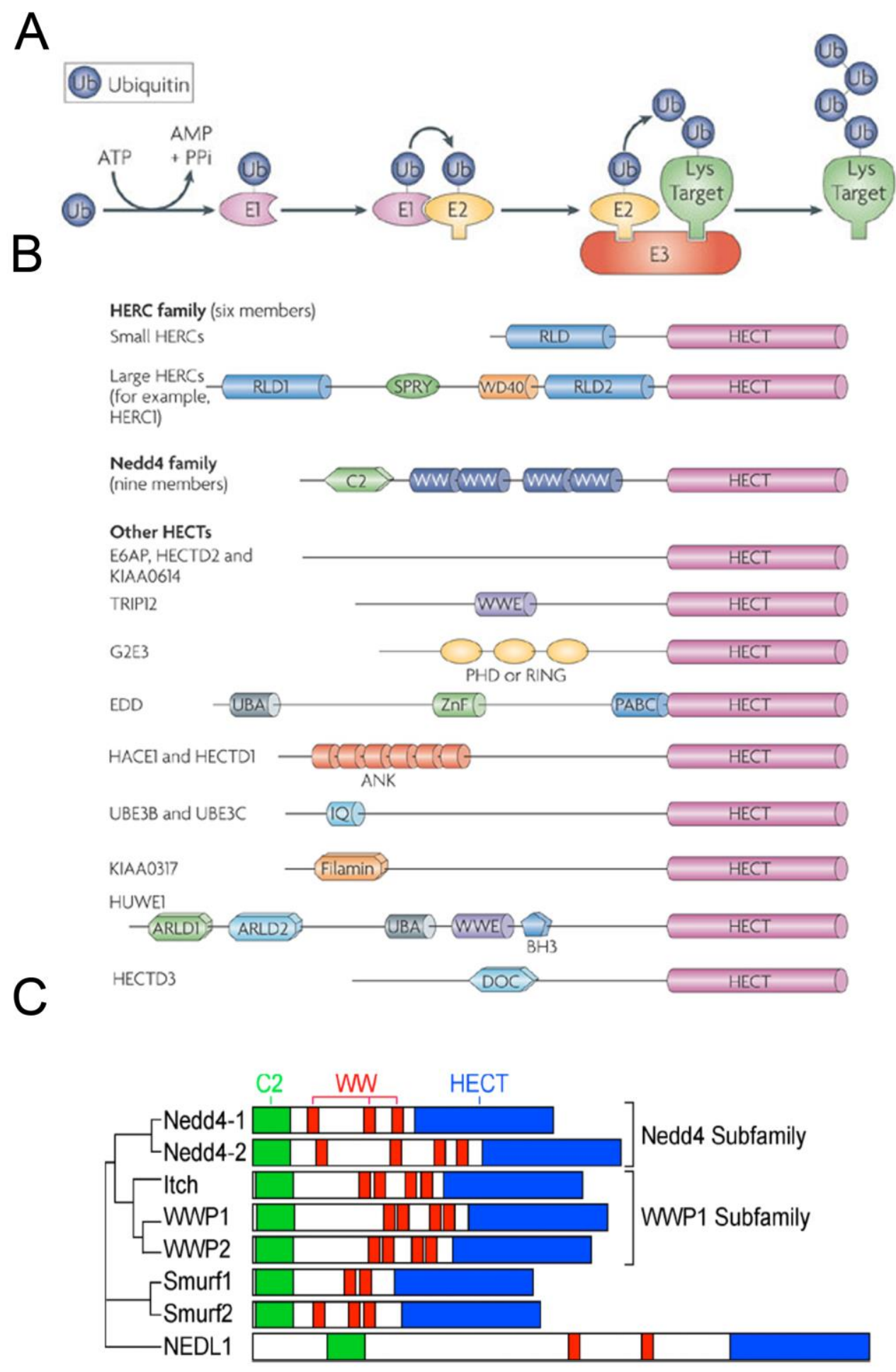

Fig. 3. HECT Type E3 ubiquitin ligases. (A) Mode of action of HECT type ligases. Ubiquitin is conjugated to E1 ubiquitin activating enzyme at the expense of ATP hydrolysis. Thereupon, ubiquitin is shuttled to E2 ubiquitin conjugating enzyme and associates with E3 ubiquitin ligase, which catalyzes ubiquitin transfer onto a substrate protein. (B) HECT-type ligases family. Based on the architecture of the N-termini and domain homology, HECT ligases are further subdivided into HERC family, Nedd4 family, and other ligases. Note ubiquitin ligase 3B, UBE3B and ubiquitin ligase 3C, UBE3C with N-terminal IQ motif. (C) 
HECT ligases of Nedd4 family. N-terminal C2 domains, depicted as green rectangles, bind calcium and mediate association with lipid rafts. WW domains, represented as red rectangles, are responsible for substrate recognition and binding of proline rich stretches in proteins. C-terminal HECT domain, shown as a blue rectangle, catalyzes ubiquitin conjugation.

(A) and (B) After [92] with permissions of Nature Publishing Group (license number 3707590499893).

\subsubsection{Nedd4 ligases in neuritogenesis}

Nedd4 ligases are essential regulators of neuronal morphology. Neural precursor cell expressed, developmentally downregulated Nedd4-1 and SMAD ubiquitination regulatory factor 1 Smurf1 are critical for proper neuritogenesis. Upon phosphorylation, Smurf1 shuttles from partitioning defective 6, Par 6 to a small GTPase RhoA at the tip of axons. Smurf1 mediates proteasomal degradation of RhoA, critical regulator of cytoskeleton stability in neurons. Overexpression of Smurf1 mutant that mimics its phosphorylation results in specification of multiple axons, while Smurf1 KD abrogates axon specification in neurons [96].

Interestingly, HECT-type ligases control neuritogenesis also by non-proteasomal degradation of their target proteins. Nedd4-1 monoubiquitylates a small GTPase Rap2. This prevents Rap2 interaction with downstream Traf2 and NCK-interacting protein, Tnik. GTPbound Rap2A-Tnik complex abrogates outgrowth of neurites. Monoubiquitylation of Rap2 by Nedd4-1 results in dissociation of Rap2A-Tnik complex and promotes neurite growth and branching. Consequently, Nedd4-1 deletion from neurons results in reduction of the dendritic tree complexity and neurite outgrowth [97]. Moreover, recent data uncovers the role of Nedd4-1 and Nedd4-2 in facilitating axon growth and branching, which in contrast to previously published reports, seems to depend on Pten-mediated regulation of Nedd4-1 mRNA translation [98].

\subsubsection{The role of Nedd4 ligases in regulation of neurotransmitter receptors, ion channels and transporters}

Dynamic regulation of synaptic proteins is essential for synaptic plasticity. By regulating the abundance, expression, subcellular localization, and function of neurotransmitter receptors, 
ion channels, or transporters, ubiquitylation contributes to structural and functional plasticity of synapses and activity of neuronal networks.

Homologous $\mathrm{Ca}^{2+}$-binding $\mathrm{C} 2$ domain, essential for association of Nedd4 ligases with lipid rafts is of particular importance for neuronal functions [95]. Recent data demonstrate that upon activation of AMPARs, Nedd4-1 translocates into dendritic spines of hippocampal neurons. This subcellular activation-induced compartmentalization appears to depend on $\mathrm{C} 2$ domain, considering the fact that Nedd4-1 mutants devoid of N-terminus fail to exhibit translocation [99]. C2 domain is essential for Nedd4-1 association with GluA1 containing

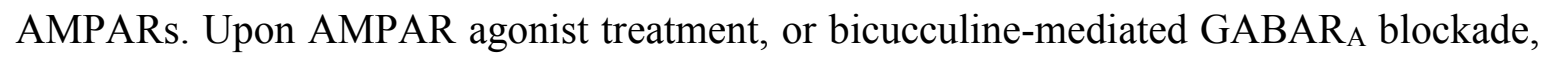
Nedd4-1 catalyzes Lys-63 linked polyubiquitin of a lysine cluster in the C-terminal loop of GluA1 and GluA2, inducing the removal of AMPARs from synaptic surface and their endosomal trafficking [100]. Moreover, Nedd4-1 mutants deficient for C2 domain are unable to reduce AMPAR-mediated synaptic transmission [99].

Nedd4-1-mediated AMPAR internalization and degradation depends on activation of voltage gated calcium channels and kinases, e.g. calcium-calmodulin kinase II, CaMKII, or serum-, and glucocorticoid regulated kinase 1, Sgk1. Upon stress, Sgk1 phosphorylates Nedd4-1, which enhances its ubiquitylation and resultant degradation of GluA1 in medial prefrontal cortex of rats [101]. Overexpression of Nedd4-1 results in decreased miniature excitatory postsynaptic currents (mEPSCs), and alters the ratio of NMDAR to AMPAR current, which corroborates Nedd4-1-mediated decreased surface expression of AMPARs [102].

Close homolog of Nedd4-1, Nedd4-2 has been reported to ubiquitylate and regulate membrane abundance of voltage gated potassium and sodium channels. In line with these studies, Nedd4-2 is essential for sodium channel function and pain processing. Genetic deletion of Nedd4-2 from dorsal root ganglion neurons in mice results in thermal hypersensitivity and allodynia [103-107].

Nedd4-2 mediates protein kinase C-induced ubiquitylation of dopamine transporter. Additionally, Nedd4-2-mediated ubiquitylation regulates surface expression of excitatory amino acid transporter 2, EAAT2, essential for regulating extracellular glutamate concentration [108-110]. Nonetheless, cell autonomous effects of Nedd4-2 on synaptic transmission has not been studied to date. 


\subsubsection{Molecular basis of Angelman syndrome and the role of Ube3a in developing and mature neurons}

Among well characterized ubiquitin ligases in terms of neuronal development and homeostasis is the founding member of HECT type ligase family, E6-associated protein, E6AP, or ubiquitin protein ligase E3A. The gene of Ube3a is subjected to genomic imprinting is mice and humans resulting in silencing of paternal Ube3a allele [111]. Molecular determinants of this silencing seem to involve attenuating of the sense Ube $3 a$ transcript by a long non-coding RNA, however, the details of this interaction remain to be elucidated [112].

Mutations in maternal Ube $3 a$ allele in humans are causative of Angelman syndrome, a debilitating neurodevelopmental disorder. Patients with Angelman syndrome exhibit severe developmental delay with intellectual disability, speech impairment, characteristic tremulous motor disturbances, particular behavioral features such as excitable personality, and happy demeanor. Frequently, patients diagnosed with Angelman syndrome display microcephaly, seizures, and abnormal EEG with large amplitude slow-spike waves [113$116]$.

Interestingly, behavioral analyses of mice deficient of maternal Ube $3 a$, which constitutes a model of Angelman syndrome reveal striking resemblance to feature of Angelman syndrome patients. Mice devoid of maternal allele of Ube3a (Ube3a KO) exhibit seizures, motor deficiencies, aberrant spatial, and contextual learning. Hippocampal activity of Ube3a KO mice monitored by electroencephalography is characterized by abnormal spikes, sharp waves, and spike-wave discharges [117,118]. On the molecular level, Ube3a KO neurons display increased phosphorylation of CaMKII, resulting in its lower activity, and lower levels of CamKII associated with postsynaptic densities [119]. Consequently, high frequency stimulation in CA1 subfield of hippocampus in the Ube $3 a \mathrm{KO}$ hippocampus results in a very transient potentiation, indicative of ineffective LTP.

In mice, $U b e 3 a$ is critical for polarized morphology of developing neurons. Knock-down of isoform 2 of Ube3a abrogates growth of apical dendrites in CA1 pyramidal neurons. Consequently, maternal Ube3a-deficient mice, mouse model of Angelman syndrome, exhibit stunted and less branched apical dendrites projected by cortical neurons of layer II/III or CA1 subfield of hippocampus [120].

Ube3a seems to localize to pre- and postsynaptic terminals of hippocampal neurons, to regulate spine morphology, and synaptic transmission [121]. Pyramidal neurons of layer 
II/III in visual cortex of Ube3a KO mice exhibit reduced density of dendritic spines concomitant with impairments of LTP and LTD, which represents an activity-dependent reduction in synaptic transmission. Interestingly, loss of plasticity and altered spine morphology observed in Ube3a KO neurons seem to rely on external stimuli. Described phenotypical changes are present in animals exposed to visual inputs, and not the ones reared in darkness [122].

At the molecular level, depletion of Ube3a decreases glutamatergic transmission, which is in line with observed decreased spine density and shrinkage of spine heads. Ube3a $\mathrm{KO}$ neurons exhibit reduced frequency of mEPSCs, indicative of decreased synapses specified on individual neurons. Interestingly, sh-RNA mediated downregulation of activity-regulated cytoskeleton-associated protein, Arc, restores AMPAR-positive synapses [123]. Arc induces endosomal recycling of AMPARs and its expression level is increased in Ube $3 a \mathrm{KO}$ neurons. Although Ube3a ubiquitylates Arc in in vitro assays, if Arc represents Ube3a substrate remains controversial [124].

Ube3a catalyzes ubiquitylation of phosphorylated ephexin-5 (E5), which acts as a Rho guanine nucleotide exchange factor and negatively regulates spine numbers in rat hippocampal neurons. Aberrant spine number and morphology might be attributable to increased neuronal levels of $\mathrm{E} 5$ reported in Ube3a KO mice [125].

Recent report describes that de novo missense mutation in autistic patients abrogates phosphorylation of UBE3A by protein kinase A, PKA, essential for inhibition of UBE3A enzymatic activity. Excessive UBE3A-mediated ubiquitylation results in abnormal excessive spine formation on primary basal dendrites of layer II/III neurons [126].

\subsubsection{HECT ligases in intellectual disability syndromes}

Disturbances in ubiquitin signaling comprise the molecular basis of numerous human diseases, such as cancer, multisystem autoimmune disease, and various brain disorders [127129]. Strikingly, malfunction HECT-type ligases has been reported for syndromes characterized with intellectual disabilities. HUWE1, located on chromosome $\mathrm{X}$ in humans, is causative for Brown-Wisniewski-Brooks syndrome, also called X-linked mental retardation [130-132]. UBE3A comprises a well characterized gene mutated in patients with Angelman syndrome, who suffer from mental retardation [133]. Interestingly, a genetic screen of sporadic mutations in patients with autism spectrum disorder reveals point mutations in $U B E 3 C$, a gene for ubiquitin ligase $\mathrm{E} 3 \mathrm{C}$ whose role in neurons has been 
uncharted [134]. Interestingly, UBE3C has been reported to associate with human postsynaptic densities [135].

Additionally, recent reports identify truncating mutations in human $U B E 3 B$ gene, encoding for ubiquitin ligase E3B, as causative for Kaufman oculocerebrofacial syndrome [136-139].

\subsubsection{Clinical symptoms of Kaufman oculocerebrofacial syndrome}

In 1971, Robert Kaufman described a peculiar pathology in four of seven siblings, he examined in Genetics Clinic at St. Louis City Hospital in Washington D.C. All of four patients exhibited severe mental retardation with absent speech, mild up-slanting of palpebral fissures, characteristic facial dysmorphisms with blepharophimosis, aberrances of the eye, ectodermal anomalies, and micrognathia, a disorder known today as oculocerebrofacial syndrome [140].

Since then, about 50 cases of Kaufman oculocerebrofacial syndrome (KOS) have been reported worldwide (OMIM \#244450). Main features of the disease include distinctive face and eye anomalies, microcephaly, severe psychomotor retardation, and growth arrest. Additionally, serum analyses unveil hypocholesterolemia, implicating defects in cholesterol metabolism. Neurological aberrances include hypoplasia of corpus callosum, and anterior commissure, Chiari type I malformation, smaller pituitary gland, and in some cases seizures. Notably, all KOS patients exhibit severe mental retardation [136-138].

The presence of blepharophimosis and mental retardation makes the KOS diagnosis challenging for physicians, as both phenotypes have been reported for other syndromes, such as Dubowitz syndrome (OMIM \#223370), Toriello-Carey syndrome (OMIM \#217980), Marden-Walker syndrome (OMIM \#248700), Ohdo syndrome (OMIM \#249620), or SmithLemli-Optiz syndrome (OMIM \#270400).

Recently the groups of Guntram Borck at University of Ulm and of Giuseppe Zampino at Universitá Cattolica del Sacro Cuore in Rome have revealed mutations in $U B E 3 B$, which inherited in autosomal recessive manner, are causative for KOS, a distinct intellectual disability syndrome [136,138]. 


\subsubsection{2. $U B E 3 B$ gene discovery}

$U B E 3 B$ gene was discovered in 1996 by the group of Margaret Lomax at the University of Michigan Medical School [141]. Differential mRNA expression study, to reveal genes upregulated after acoustic trauma in the chick basal papilla, lead to identification of cDNA which exhibited $84 \%$ of identity of uncharacterized human cDNA. Interestingly, its expression dramatically increased in the regions of damaged chick inner ear upon noiseinduced trauma. In 2003, human and mouse $U B E 3 B$ gene was cloned and characterized by its discoverers [142]. Degree of homology of HECT domains between UBE3B and UBE3C is higher than between other member of HECT type, implicating a distinct biochemical properties and ubiquitylation patterns catalyzed by both ligases. For this reason, UBE3B and UBE3C represent a separate family of HECT-type ligases.

\subsubsection{Mutations in $U B E 3 B$ identified in KOS}

Pathological mutations in $U B E 3 B$ in KOS patients appear to affect the enzymatic activity of the ligase. Majority of reported mutations result in frame shift, leading to loss of catalytic function of UBE3B Interestingly, substitution mutations have been reported as well. All reported point mutations affect amino acids of HECT domain, implicating conformational restrictions hampering UBE3B-mediated ubiquitylation. In fact, three dimensional modeling of UBE3B HECT domain illustrates that Q727P substitution, identified in one of KOS patients may affect substrate binding and positioning towards catalytic cysteine, essential for efficient ubiquitylation [136,138,139,143].

Although UBE3B contribution to human disease has been reported by at least four independent research groups, its molecular function still remains uncharted.

\subsection{Posttranscriptional regulation of gene expression: biology of microRNAs}

Precise control over gene expression is critical for uncompromised neuronal development. Small non-coding RNAs, microRNAs (miRNAs) are key regulators of gene expression. miRNAs, expressed in the majority of Eucaryota, mediate sequence-specific posttranscriptional gene regulation. As discovered by Victor Ambros and Gary Ruvkun in December 1993, miRNA-mediated regulatory mechanism involves binding to complementary RNA sequences, usually located in the 3' untranslated regions of messenger 
RNAs (mRNA) [144,145]. Intriguingly, most coding genes seem to be regulated by miRNAs [146].

\subsection{1. miRNA gene transcription and initial processing of miRNA precursors}

Transcription of miRNA gene locus is majorly carried out by RNA-polymerase II and yields an approximately $1 \mathrm{~kb}$ long, primary miRNA transcript, pri-miRNA with local hairpin-loop structures. Microprocessor complex including RNase III, Drosha and its cofactor DiGeorge syndrome Critical Region gene 8 protein (DGCR8) cleaves pri-miRNA into $60-70$ nucleotide-long precursors (pre-miRNA) [147]. Drosha processing of pri-miRNA is critical for mature miRNA specificity, because it defines the terminal nucleotides of each miRNA strand. The resultant pre-miRNA contains a two nucleotide-long overhang on its 3 ' terminus, essential for its further processing by Dicer [148-150]. Drosha determines miRNA abundance, therefore, mechanisms controlling Drosha expression, stability, or activity control the levels of miRNAs. Processing of pri-miRNAs is regulated by RNA-binding proteins, which affect recruitment of Drosha to the terminal loops of miRNA precursor, containing numerous $c i s$-regulatory sequences $[151,152]$.

\subsubsection{Nucleus-cytoplasm shuttle of pre-miRNAs}

Initial processing of the miRNA precursors is restricted to nucleus. Upon exportin-5mediated nuclear transport, pre-miRNAs are further processed in the cytoplasm by RNase III Dicer and its cofactor TAR RNA-binding protein 2 (TRBP2). Exportin-5 forms a complex with the pre-miRNA and Ran-GTP, a nuclear GTPase. Upon translocation through the nuclear pore, GTP is hydrolyzed, and pre-miRNAs are released to the cytosol [153]. Transport of non-canonical miRNAs, like pre-mir-320, which contains 5' 7-methylguanylate cap, is carried out by exportin-1 [154]. 


\subsubsection{Cytosolic pre-miRNA processing}

Dicer processing removes the terminal loop of the pre-miRNA to liberate a miRNA duplex composed of two complementary strands: $-5 p$ and $-3 p$. Dicer binds to pre-miRNA with the two-nucleotide overhangs, generated previously by Drosha [150]. Dicer domains critical for its RNase III activity are positioned so, that the cleavage of pre-miRNA liberates the mature miRNA strands of a 21-25 nucleotides in length, depending on the Dicer isoform. Therefore, the distance between those domains functions as a 'molecular ruler' in miRNA processing [155].

\subsubsection{Formation of RNA-induced silencing complex}

Generally, after Dicer processing, miRNA duplex associates with Argonaute (Ago) to form a RNA-induced silencing complex (RISC). RISC is able to regulate translation of a target mRNA in a sequence-specific manner $[156,157]$. Small miRNA duplexes are loaded into RISC in ATP-dependent manner [158], mediated by conformational changes of Dicer induced by heat shock cognate 70, HSC70, and heat shock protein 90, HSP90 chaperones [159]. Mismatches in the center of the duplex prevent the endonucleolytic activity of Ago proteins and are essential for duplex unwinding, which releases passenger strand and biologically active guide strand [160]. Release of passenger strand from miRNA duplex is ATP-independent [161].

\subsubsection{Strand selection and target $m R N A$ recognition}

Determination of the guide and passenger strand relies on the thermodynamic stability of the two termini of miRNA duplex [162]. Strand with more unstable 5' end is typically specified as the guide strand. Interestingly, arm switching, a process of alternative strand selection has been described for some miRNA duplexes[163].

Main determinants of miRNA-mRNA binding include perfect complementarity between mRNA and miRNA nucleotides 2 to 8 , so called miRNA seed. Complementarity between 3' terminus of a passenger strand and mRNA is critical for stabilization of miRNA-mRNA hybrid [164,165]. Although the canonical miRNA-mediated gene regulation leads to translational arrest, under certain cellular contexts, miRNAs are known to enhance gene expression [166]. 


\subsubsection{Molecular mechanisms of mRNA silencing}

Generally, the consequences of miRNA-RISC-mRNA interaction affect the initiation and efficiency of transcription, but also alter the stability of mRNA. Perfect complementarity between miRNA and its binding site on a target mRNA induces its Ago2-mediated endonucleolytic cleavage and destabilization. Core protein components of RISC, Ago and trinucleotide repeat containing 6a, also known as GW182 are essential for miRNA-induced gene repression.

Generally, miRNA-dependent translation regulation requires mismatches in the central region of miRNA-mRNA hybrid [165]. miRNA-RISC recruit decapping enzymes (e.g. decapping protein 1, DCP1), deadenylases (e.g. CCR-associated factor 1-carbon catabolite repression 4 protein-negative on TATA-less, CAF1-CCR4-NOT complex), or nucleases, all resulting in mRNA destabilization and decay [167]. Moreover, Argonaute molecules compete with cap-binding proteins and eukaryotic translation initiation factor 4E for binding of 5' mRNA cap and hamper ribosome recruitment to the cap [168]. Association of Ago with mRNA cap prevents circularization of mRNA, essential for translation initiation [169]. Post-initiation mechanisms of miRNA-mediated gene regulation include RISC-induced premature mRNA-ribosome dissociation [170], or competition of Ago with elongation factors required for efficient mRNA translation [171] (Fig. 4B). 

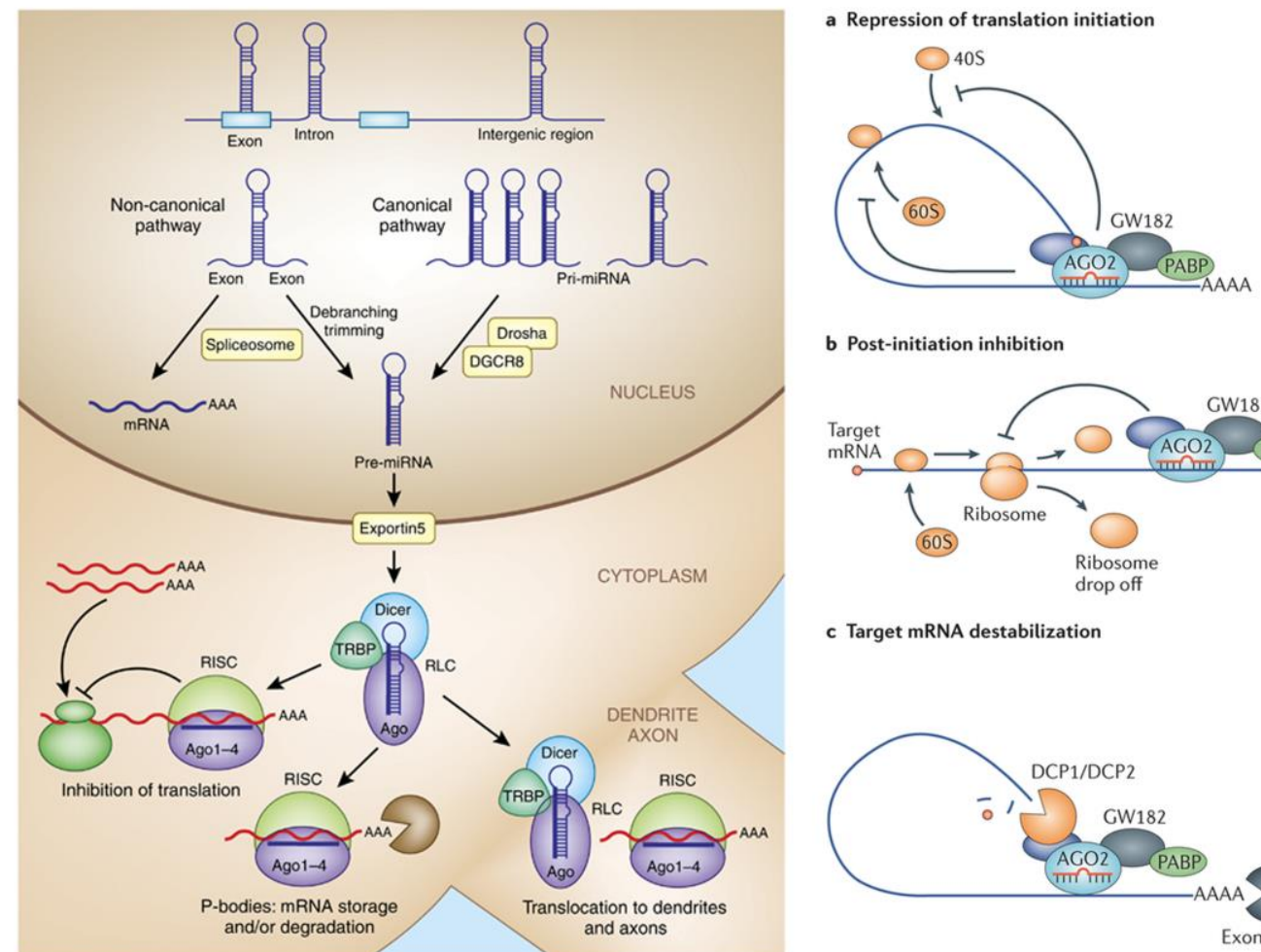

b Post-initiation inhibition

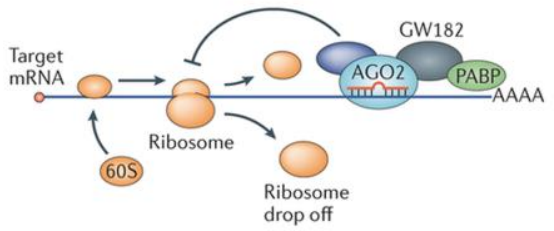

c Target mRNA destabilization

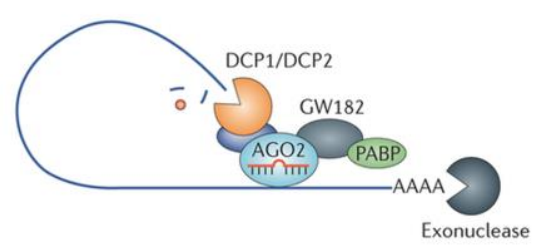

C

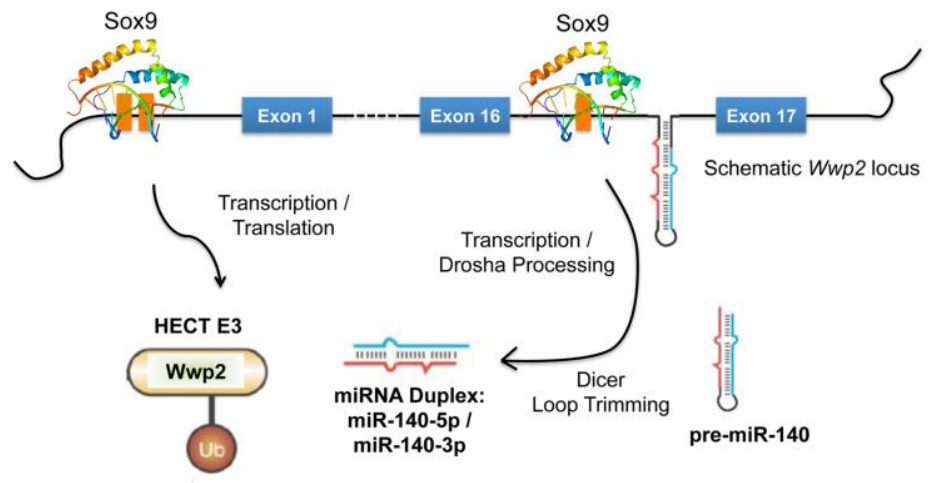

| Promoter binding sites

Fig. 4. Processing and posttranscriptional regulation of gene expression by miRNAs. (A) Processing of miRNA precursors. Upon RNA-polymerase II transcription, precursors of miRNA (pri-miRNA) are either excised by Drosha and its cofactor DiGeorge syndrome critical region gene 8 (DGCR8) in canonical processing pathway, or processed in pre-mRNA splicing (mirtrons) by debranching and trimming enzymes in non-canonical pathway. Resultant pre-miRNAs are exported to the cytoplasm by exportin-5, where they are further cleaved by Dicer, unwound by Argonaute (Ago) proteins and undergo TAR RNA-binding protein, TRBP-dependent loading onto RNA-induced silencing complex (RISC). RISC mediates the posttranscriptional gene silencing. Transport of RISC loading complex, RLC to different neuronal compartments, e.g. axons or dendrites, enables local regulation of translation. (B) Three mechanisms of miRNA-mediated gene regulation. (a) Repression of translation initiation. RISC prevents circularization of mRNA critical for translation initiation. Argonaute-2, Ago2; trinucleotide repeat containing 6a, GW182; poly-A binding 
protein, PABP. (b) miRNA-mediated inhibition of elongation. RISC induced premature drop-off of ribosome from mRNA and binding of elongation factors critical for active translation. (c) Destabilization of target mRNA by miRNAs. RISC recruits decapping enzymes 1 and 2 (Dcp1 and DCP2), that hydrolyze 5'cap, and deadenylases that mediate destruction of polyA tail, and recruit exonucleases. (C) Schematic of $W w p 2$ gene locus. Intron 16 of $W w p 2$ harbors $m i R-140$ gene. SRY-box containing gene 9, Sox 9 transcription factor binding sites are located upstream of $W w p 2$ exon 1 and within intron 16, upstream of $m i R-140$. Upon Sox9-mediated transcription, translation and posttranscriptional processing, Wwp2 locus simultaneously generates a ubiquitin ligase and miR-140 [172].

(A) After [173] with permissions of Nature Publishing Group (license number 3707680449640). (B) After [174] with permission of Nature Publishing Group (license number 3707620640588 ).

\subsubsection{The role of Dicer in developing and mature neurons}

The crucial importance of miRNAs during neuronal development is illustrated by studies on Dicer KO mice. Dicer-mediated processing represents essential step of miRNA biogenesis. Conditional neuron- and glia-specific, Emxl-Cre driven knock-out (cKO) of Dicer results in defects of gross morphology on the brain including ventricular dilatation and reduction in cortical thickness from E13.5 onwards [175].

In postmitotic neurons, Dicer ablation leads to abnormal neuronal migration resulting in reduction of upper cortical layers thickness and lack of deep cortical layers. Conditional deletion of Dicer impedes radial migration and disrupts polarity formation in newborn neurons. Dicer KO results in emergence of multipolar cells throughout cortical plate, while migrating control neurons mostly acquire bipolar morphology [176,177]. Additionally, Dicer-deficient pyramidal neurons of the hippocampus exhibit reduced dendrite complexity and increased dendritic spine length [178].

Pathological changes in morphology of Dicer-deficient neurons are paralleled by pathological behavioral outcomes. CaMKII-driven Dicer cKO mice display ataxia, motilityinduced tremors and early postnatal death [178]. Deletion of Dicer from the dopaminergic neurons of basal ganglia using dopamine receptor-1-Cre results in front and hind limb clasping in mice, indicative of motor impairments and neurological pathology [179].

Collectively, genetic ablation of Dicer and resultant reduction of mature miRNA levels lead to dramatic morphological and physiological consequences, indicating that Dicer-mediated miRNAs processing is essential for developing and mature neurons. 


\subsubsection{Biology of intragenic miRNAs}

Interestingly, about half of murine miRNA loci are located within introns of protein-coding genes, thereby linking miRNA gene transcription to host gene promoters [180]. However, $35 \%$ of known intronic miRNAs have upstream regulatory elements, such as RNApolymerase start sites, compatible with promoter function. This indicates that transcription of intronic miRNA can either employ the promoter sequences of their host genes, or exploits its own intron-embedded promoters [181].

Intronic miRNAs (sometimes described as mirtrons) can be generated during pre-mRNA splicing and bypass Drosha processing [182]. Interestingly, it has been proposed, that intronic miRNAs function to fine-tune the activity of proteins encoded by their host genes [183]. Interestingly, $20 \%$ of intronic miRNAs are predicted to regulate the expression of their host [184].

\subsubsection{Interplay of Wwp2 ubiquitin ligase and intragenic miR-140 in control of gene expression}

Intron 16 of $W w p 2$, a murine gene encoding for WW domain containing E3 ubiquitin protein ligase 2 harbors a locus of $m i R-140$ (Fig. 4C). Wwp2 is a HECT-type ubiquitin ligase of Nedd4 family with well characterized roles in cancer and embryonic stem cells [185-189]. Notably, intronic miR-140 has been reported as a tumor suppressor [190-195]. Acting antagonistically to the host Wwp2, miR-140 represses SMA and mothers against decapentaplegic 2, Smad2, limiting invasive potential of colorectal cancer [196].

The biology of miR-140 has been extensively studied in cartilage. Genetic deletion of $m i R$ 140 in mice leads to dramatic proteoglycan loss, fibrillation of articular cartilage and osteoarthritis-like changes [197].

Induction of expression of C-terminal isoform of Wwp2 and miR-140 is critical for maintenance of proliferative activity of chondroprogenitor cells [172]. This implicates synergistic activities of Wwp2 and miR-140 in cartilage biology. Moreover, promoters of genes encoding both molecules are major targets of SRY-box containing protein-9, Sox 9 in cartilage [198] (Fig. 4C). Although the role of Wwp ubiquitin ligases in mature neurons remain unexplored, during neuronal development Wwp2 and its close homolog Wwp1 regulate axon acquisition in primary hippocampal neurons, polarity formation of cortical pyramidal neurons and distribution of layer II/III pyramidal nerve cells [199]. Expression of 
$m i R-140$ in the brain has been reported [200], however, its function in neurons has never been studied.

\subsection{IN BRIEF}

In this study, we characterized miR-140 and Wwp2 duo in developing neurons. We corroborate that Wwp1 and Wwp2 are indispensable for axon acquisition in developing hippocampal neurons and neuronal polarity of cortical neurons, and that knock-down of Wwp1 and Wwp2 in cortical progenitors results in aberrant distribution of pyramidal neurons. Moreover, we present that miR-140 exerts synergistic effects towards Wwp1 and Wwp2 activities during neuronal development. Genetic deletion of miR-140 results in disturbances of axon acquisition and dramatic defects in polarity formation in cortical neurons. Moreover, we demonstrate that acute knock-down of miR-140-3p in neurons phenocopies knock-down of Wwp1 and Wwp2 concerning neuronal development. Finally, for the first time, we identify Sox 9 as a transcription factor essential for neuronal polarity and neuronal distribution of postmitotic pyramidal neurons, implicating Sox9-Wwp1/2miR-140 axis in control of neuronal development.

We characterize the roles of other HECT-type ubiquitin ligases in developing and mature neurons. We demonstrate, that genetic deletion of Nedd4-2, and Wwpl and Wwp2 conditionally in neurons leads to alterations of dendritic spine morphology and/or number, which implies that enzymes of Nedd4 family are critical for uncompromised function of mature neurons.

For the first time, we characterize murine $U b e 3 b$ in neurons. We generated a conventional and neuron- and glia-specific Ube $3 b \mathrm{KO}$ mouse lines, both recapitulating symptoms described for KOS patients. We demonstrate that Ube3b is essential for developing and mature neurons. Primary hippocampal neurons deleterious for $U b e 3 b$ exhibit reduced dendritic branching, which is reversible by overexpression of human UBE3B, but not by UBE3B with point substitution mutations identified in KOS patients. Moreover, Ube $3 b$ loss from pyramidal hippocampal neurons leads to increased density of dendritic spines, which exhibit defectful morphology. Consistently, we show that conditional Ube3b deletion from neurons leads to increased mEPSC frequency and alters NMDAR to AMPAR ratio, indicative of increased synapse number and composition of glutamate receptors specified by neurons deleterious for Ube3b. In line with our cell biological and electrophysiological 
findings, we demonstrate that neuron- and glia-specific Ube $3 b \mathrm{cKO}$ animals display impairment in spatial learning and aberrant social interactions.

Altogether, this study corroborates essential role of HECT-type ligases in neurons and provides novel insights into the molecular mechanisms of neuronal development in health and disease. 


\section{MATERIALS AND METHODS}

\subsection{Animals}

Colonies of wild type mice has been maintained in the animal facility of Max Planck Institute of Experimental Medicine (MPIem) and all used in this study unless stated otherwise were of C57BL/6N strain. $W w p 1^{\mathrm{f} / \mathrm{f}} ; W w p 2^{\mathrm{f} / \mathrm{f}}$ and Nedd4-2 $2^{\mathrm{f} / \mathrm{f}}$ mouse lines were generated by Dr. Hiroshi Kawabe and Prof. Nils Brose. Prof. Tatsuya Kobayashi kindly provided the $m i R$ 140-/- line [201], NEX1-Cre mouse line was from Dr. Sandra Goebbels and Prof. KlausArmin Nave [202], and Emxl-Cre was kindly shared by Dr. Yuqing Li [203]. Sox $9^{\mathrm{f} / \mathrm{f}}$ mice were originally generated by Prof. Haruhiko Akiyama's group, and PFA-fixed brains of Sox $9^{\mathrm{f} / \mathrm{f}}$ after in utero electroporation were provided by Dr. Mikio Hoshino. Ube $3 b$ mutant ES cells were purchased from The European Conditional Mouse Mutagenesis Program (EUCOMM), and $U b e 3 b^{-/-}$, and $U b e 3 b^{\mathrm{f} / \mathrm{f}}$; Emxl-Cre lines were established during this study. All animal experiments have been performed in compliance with welfare guidelines issued by the state of Lower Saxony. Corresponding permits for animal experiments have been granted (33.9-42502-04-13/1359, and 33.19-42502-04-13/1052).

\subsection{Bacterial and yeast strains}

Table 1. Bacteria and yeast used during this study.

\begin{tabular}{|l|l|}
\hline E. coli XL-1 Blue competent cells & Stratagene \\
\hline E. coli Electro10-Blue competent cells & Stratagene \\
\hline E. coli JM109 competent cells & Promega \\
\hline E. coli TOP10 competent cells & Invitrogen \\
\hline S. cerevisiae PJ69-4A strain (James et al., 1996) & Invitrogen \\
\hline
\end{tabular}

\subsection{DNA}

DNA used or created during this study is listed in tables below. 
Table 2. cDNA libraries used in this study.

\begin{tabular}{|l|l|}
\hline Mouse brain prey library (in pGAD-GL) & Provided by Dr. Hiroshi Kawabe \\
\hline Rat brain prey library (in pVP16-3) & Provided by Prof. Nils Brose \\
\hline P0 mouse cortex library & Generated in this study \\
\hline
\end{tabular}

Table 3. Vector plasmids used in this study.

\begin{tabular}{|c|c|}
\hline ENV & Provided by Prof. Pavel Osten \\
\hline $\mathrm{L} 21$ & Provided by Prof. Pavel Osten \\
\hline PACK & Provided by Prof. Pavel Osten \\
\hline pcDNA-3.2-GW/EmGFP & Invitrogen \\
\hline pcDNA-3.2-GW/EmGFP-miR-140 & Generated in this study \\
\hline pcDNA-3.2-GW/EmGFP-miR-140 & Generated in this study \\
\hline pcDNA-3.2-GW/EmGFP-Scramble & Invitrogen \\
\hline pcDNA3-HA-hUbe3b & $\begin{array}{l}\text { Provided by Rüstem Yilmaz and Prof. Guntram } \\
\text { Borck }\end{array}$ \\
\hline pcDNA3-HA-hUbe3b G779R & $\begin{array}{l}\text { Provided by Rüstem Yilmaz and Prof. Guntram } \\
\text { Borck }\end{array}$ \\
\hline pcDNA3-HA-hUbe3b R997P & $\begin{array}{l}\text { Provided by Rüstem Yilmaz and Prof. Guntram } \\
\text { Borck }\end{array}$ \\
\hline $\mathrm{pCIG}$ & Provided by Dr. Evangelos Pavlakis \\
\hline pCIG-2XmiR-140 & Generated in this study \\
\hline pCR2.1-HECT-Nedd4-2 & Generated in this study \\
\hline pCR2.1-HECT-Ube3b & Generated in this study \\
\hline pCR2.1-IQ-Ube3b & Generated in this study \\
\hline pCR2.1-IQ-Ube3b & Generated in this study \\
\hline pCR2.1-SB-3'Ube3b & Generated in this study \\
\hline pCR2.1-TOPO & Invitrogen \\
\hline pCR2.1-Ube3b (WT, full length) & Generated in this study \\
\hline pCR2.1-Ube3b C1038S & Generated in this study \\
\hline pCR2.1-Wwp2/miR-140 & Generated in this study \\
\hline pCX::myrVenus & Provided by Dr. Anna-Katerina Hadjantonakis \\
\hline pFUGW & Provided by Dr. Inder Verma \\
\hline pFUGWiCre & Provided by Dr. Richard Huganir \\
\hline pGAD424-HA & Clontech \\
\hline pGBD-C2 & Provided by Dr. Philip James \\
\hline pGBD-IQ-Ube3b & Provided by Dr. Hiroshi Kawabe \\
\hline pLKO.1-Scramble & Thermo Scientific \\
\hline pLKO.1-sh-mSox9-2 & Addgene, gift of Bob Weinberg, [204] \\
\hline pLKO.1-sh-mWwp1 & Thermo Scientific \\
\hline pLKO.1-sh-mWwp2 & Thermo Scientific \\
\hline pLKO.1-Venus-Scramble & Provided by Dr. Hiroshi Kawabe \\
\hline
\end{tabular}




\begin{tabular}{|l|l|} 
pLKO.1-Venus-sh-mSOX9 & Generated in this study \\
\hline pLKO.1-Venus-sh-mWwp1 & Provided by Dr. Hiroshi Kawabe \\
\hline pLKO.1-Venus-sh-mWwp2 & Provided by Dr. Hiroshi Kawabe \\
\hline pCAG & Provided by Dr. Jun-ichi Miyazaki \\
\hline pCAG-CTR-Sponge & Generated in this study \\
\hline pCAG-miR-140-3p-Sponge & Generated in this study \\
\hline pCAG-miR-140-5p-Sponge & Generated in this study \\
\hline pCAG-myc-1 & Provided by Dr. Hiroshi Kawabe \\
\hline pCAG-myc-IQ-Ube3b & Generated in this study \\
\hline pCAG-myc-Ube3b & Generated in this study \\
\hline pCAG-myc-Ube3b C1038S & Generated in this study \\
\hline pVP-16-3 & Provided by Prof. Nils Brose \\
\hline pWPXL-Sox9 & Addgene, gift of Bob Weinberg, [204] \\
\hline pYX-Asc-Ube3b & BioCat \\
\hline
\end{tabular}

\subsection{Oligonucleotides}

Oligonucleotides used in this study are listed below. All of them were synthetized in MPIem DNA Core Facility. Reaction sites employed for molecular cloning are underlined.

Table 4. Oligonucleotides used in this study (Nb, number; $R S$, restriction site).

\begin{tabular}{|c|c|c|}
\hline $\mathbf{N b}$ & Sequence (5'-3') & $\underline{\mathbf{R S}}$ \\
\hline 89 & 5'-TGTAAAACGACGGCCAGT -3' & - \\
\hline 91 & 5'-AACAGCTATGACCATGATTACG -3' & - \\
\hline 2409 & 5'-CCGCATAACCAGTGAAACAG -3' & - \\
\hline 8546 & 5'-GCCTGCATTACCGGTCGATG -3' & - \\
\hline 10908 & 5'-AGAGGCAAGAGAATGGCGTCAAG -3' & - \\
\hline 10909 & 5'-CAGAAACAGTGATCTACAAGTCTAAATG -3' & - \\
\hline 12804 & 5'-CTCCCCACTGCAGTTCCTACC -3' & - \\
\hline 12806 & 5'-AGCTGCTCAGGCTGAATCACC -3' & - \\
\hline 12809 & 5'-CCATCATGAACTTCAGCCTC -3' & - \\
\hline 12812 & 5'-GATGGTTGTGAGCCACTTACTTC -3' & - \\
\hline 14539 & 5'-CAATGAATACGGCTACAGCAAC -3' & - \\
\hline 14540 & 5'-TTACTCCTTGGAGGCCATGT -3' & - \\
\hline 18637 & 5'-GTTTCTGGTCCTCACATTGTCTA -3' & - \\
\hline 18638 & 5'-ATTTCCGGTTATTCAACTTGCAC -3' & - \\
\hline 19902 & 5'-GCCTGAGGGCAGTAAGGAC -3' & - \\
\hline 19903 & 5'-GGAGTAGGGCGGCTCTGT -3' & - \\
\hline 25252 & 5'-ATGCCCAGAAGGTACCCCAT -3' & - \\
\hline 25253 & 5'-TTTCCGGTTATTCAACTTGCACCA -3' & - \\
\hline
\end{tabular}




\begin{tabular}{|c|c|c|}
\hline 25831 & $\begin{array}{l}\text { 5'- } \\
\text { TGCTGCAGTGGTTTTACCCTATGGTAGGTTTTGGCCACTG } \\
\text { ACTGACACCACAGGAGAACCACGGAC -3' }\end{array}$ & - \\
\hline 25832 & $\begin{array}{l}5^{\prime}- \\
\text { CCTGGTCCGTGGTTCTCCTGTGGTGTCAGTCAGTGGCCA } \\
\text { AAACCTACCATAGGGTAAAACCACTGC -3' }\end{array}$ & - \\
\hline 25939 & 5'-CAGAGCACTGTGTTACCTTCACCC -3' & - \\
\hline 25940 & 5'-CCTAACCCAGATTTGGATCCATTTAT -3' & - \\
\hline 26082 & 5'-GACTGGAGCTCCAGAGCACTGTGTTACCTTCACCC -3' & SacI \\
\hline 26083 & 5'-GATCGCTCGAGCAGAGCACTGTGTTACCTTCACCC -3' & Xhol \\
\hline 26084 & $\begin{array}{l}\text { 5'-CATGCGAGCTCCCTAACCCAGATTTGGATCCATTTAT - } \\
\text { 3' }\end{array}$ & SacI \\
\hline 26326 & $\begin{array}{l}5^{\prime}- \\
\text { GAGGAATTCACCATGTTCACTGTATCTCAGACCTCCAGA } \\
\text { GC -3' }\end{array}$ & EcoRI \\
\hline 26327 & 5'-CTCCTCGAGTTATTAGGAGAGCTCAAAGCCCGTGTT -3' & XhoI \\
\hline 26395 & 5'-CTC萳GAGTTACGCAGCAACCACAGGGCG -3' & XhoI \\
\hline 26703 & $\begin{array}{l}\text { 5'- } \\
\text { GGCGTCTGCCCACATCTTCCACTAGCTTCAACCTGCTTAA } \\
-3^{\prime}\end{array}$ & - \\
\hline 26704 & $\begin{array}{l}\text { 5'- } \\
\text { TTAAGCAGGTTGAAGCTAGTGGAAGATGTGGGCAGACG } \\
\text { CC -3' }\end{array}$ & - \\
\hline 27678 & 5'-TTAGTGTGGCTTTTCAGCCTTAA -3' & - \\
\hline 27679 & 5'-TGGAGCCGTTAGGTCATTTCA -3' & - \\
\hline 27879 & 5'-GGGGGCGTGGCCAGAGAAT -3' & - \\
\hline 27880 & 5'-CACGTCGACATCACCAAGGCC -3' & - \\
\hline 27883 & 5'-CGGATGTTGGAGGACGGCTA -3' & - \\
\hline 27884 & 5'-CTTGATGATGGAACGGAAGCC -3' & - \\
\hline 28441 & 5'-CCACAACGGGTTCTTCTGTTAG -3' & - \\
\hline 28741 & 5'-GTGAGAGGCACAAGTTGGC -3' & - \\
\hline 28742 & 5'-CTCTGTGTAGGATCTTCAATC -3' & - \\
\hline 28745 & 5'-CTTGGTGGGCATCTGGTGTGGCTC -3' & - \\
\hline 28746 & 5'-GAGCTAAACAATTGGGGAACAATC -3' & - \\
\hline 29127 & 5'-GTATCTTATCATGTCTGGATCCGGGGG -3' & - \\
\hline 29156 & 5'-ATGCTGGCAGACTTTGCACTCTTTACTCTC -3' & - \\
\hline 29244 & 5'-TCGGGTGTTTACTTGGATAACTCT -3' & - \\
\hline 29245 & 5'-TGTGCTTTGGTTCCTTATCTGTC -3' & - \\
\hline 31998 & 5'-CTGGGAAGTGTGCCTAATGG -3' & - \\
\hline 31999 & 5'-GCAGTCCCACTGGATTCTCT -3' & - \\
\hline 32140 & 5'-GTTGCTGCCAGACCCAAA -3' & - \\
\hline 32141 & 5'-TAGGACAGATGATGATTCTCCATTA -3' & - \\
\hline
\end{tabular}




\subsection{Antibodies}

Table 5. Primary antibodies used for Western blotting and immuoblotting used in this study (order on the list according to emergence of figures)

\begin{tabular}{|c|c|c|c|c|c|}
\hline \multirow{2}{*}{$\begin{array}{l}\text { Recognized protein } \\
\text { (clone number) }\end{array}$} & \multirow{2}{*}{ Host species } & \multirow{2}{*}{ Developer } & \multicolumn{3}{|c|}{ Usage and dilution } \\
\hline & & & WB & ICC & IHC \\
\hline SMI312 & Mouse & Millipore & - & $1: 2000$ & - \\
\hline MAP2 & Chicken & Novus & - & $1: 1000$ & - \\
\hline GFP & Rabbit & MBL & - & - & $1: 2000$ \\
\hline Wwp1 (0221) & Rabbit & SySy & $1: 500$ & - & - \\
\hline Wwp2 (7425A) & Rabbit & SySy & $1: 500$ & - & - \\
\hline$\alpha-\operatorname{actin}(\mathrm{AC} 40)$ & Mouse & Sigma & $1: 2000$ & - & - \\
\hline Cux1 (M-222) & Rabbit & SCBT & - & - & $1: 500$ \\
\hline PSD-95 & Mouse & NeuroMab & $1: 1000$ & - & - \\
\hline RabGDI & Rabbit & SySy & $1: 1000$ & - & - \\
\hline NFM 150 & Rabbit & Chemicon & $1: 1000$ & - & - \\
\hline Fyn (15) & Mouse & SCBT & $1: 500$ & - & - \\
\hline Sox9 (5535) & Rabbit & Millipore & $1: 2000$ & $1: 500$ & $1: 500$ \\
\hline Ctip2 (25B6) & Rat & Abcam & - & - & $1: 500$ \\
\hline Cre & Rabbit & SySy & - & - & $1: 1000$ \\
\hline Ube3b (339003) & Rabbit & SySy & $1: 1000$ & - & - \\
\hline Myc (9E19) & Mouse & Sigma & - & $1: 500$ & - \\
\hline HA & Mouse & Covance & - & $1: 1000$ & - \\
\hline NeuN & Mouse & Millipore & - & - & $1: 400$ \\
\hline GFAP & Mouse & SySy & - & - & $1: 1000$ \\
\hline Iba1 & Rabbit & SySy & - & - & $1: 2000$ \\
\hline MAC3 & Rat & Abcam & - & - & $1: 1000$ \\
\hline Ppp3cc & Rabbit & Thermo & $1: 500$ & - & - \\
\hline
\end{tabular}


Table 6. Secondary antibodies used for Western blotting and immunostaining used in this study

\begin{tabular}{|l|l|l|l|l|}
\hline $\begin{array}{l}\text { Recognized } \\
\text { IgG }\end{array}$ & $\begin{array}{l}\text { Host } \\
\text { species }\end{array}$ & $\begin{array}{l}\text { Conjugated } \\
\text { substrate/Fluorophore/Dye }\end{array}$ & Origin & Dilution \\
\hline Mouse & Goat & HRP & Bio-Rad & $1: 10000$ \\
\hline Rabbit & Goat & HRP & Bio-Rad & $1: 10000$ \\
\hline Mouse & Goat & IR-Dye-680/800 & Rockland & $1: 8000$ \\
\hline Rabbit & Goat & IR-Dye-680/800 & Rockland & $1: 8000$ \\
\hline Chicken & Donkey & AlexaFluor-633 & Invitrogen & $1: 1000$ \\
\hline Rat & Goat & AlexaFluor-555 & Invitrogen & $1: 1000$ \\
\hline Mouse & Goat & $\begin{array}{l}\text { AlexaFluor- } \\
488 / 555 / 633 / C y 5.5\end{array}$ & Invitrogen & $1: 1000$ \\
\hline Rabbit & Goat & $\begin{array}{l}\text { AlexaFluor- } \\
488 / 555 / 633 / C y 5.5\end{array}$ & Invitrogen & $1: 1000$ \\
\hline
\end{tabular}

\subsection{Molecular biology methods}

\subsubsection{Electroporation of plasmid DNA into competent bacteria}

An aliquot of electro-competent bacteria of a given E. coli strain was thawn on ice, transferred to pre-cooled electroporation cuvette $(0.1 \mathrm{~cm}$, Bio-Rad $)$ and $10 \mathrm{ng}$ of DNA or $0.8 \mu \mathrm{L}$ of ligation reaction was added, mixed, and incubated for 1 minute prior to electroporation. Next, an electric pulse of $1.8 \mathrm{kV}$ was administered (Electro-Pulser, Bio$\mathrm{Rad})$ and electroporated cells retrieved from the cuvette with $250 \mu \mathrm{L}$ of pre-warmed $\left(37^{\circ} \mathrm{C}\right)$ SOC medium and incubated with moderate shaking for 1 hour at $37^{\circ} \mathrm{C}$ allowing for the expression of resistance genes. Bacterial cells were then plated on appropriate selection agar plates.

LB medium: $25 \mathrm{~g}$ Luria Broth (LB, Invitrogen) dissolved in $1 \mathrm{~L} \mathrm{ddH}_{2} \mathrm{O}$ and autoclaved.

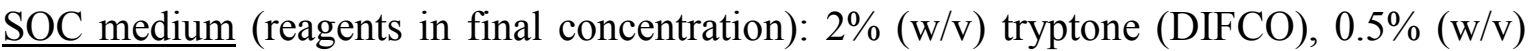
yeast extract (DIFCO), $10 \mathrm{mM} \mathrm{NaCl}, 2.5 \mathrm{mM} \mathrm{KCl}, 20 \mathrm{mM} \mathrm{MgCl}_{2}$ in $\mathrm{ddH}_{2} \mathrm{O}$, autoclaved. Before usage add glucose (filtered, $0.22 \mu \mathrm{m}$, Corning) to $20 \mathrm{mM}$.

LB agar: $15 \mathrm{~g}$ Bacto-agar (DIFCO) dissolved in $1 \mathrm{~L}$ of LB medium, autoclaved. For selection of transformed E. coli, ampicillin or kanamycin was added after autoclaving at the concentration of $50 \mu \mathrm{g} / \mathrm{mL}$ and $25 \mu \mathrm{g} / \mathrm{mL}$, respectively. 


\subsubsection{Plasmid DNA purification}

Purification of plasmid DNA from bacteria was carried out following manufacturer's protocols using PureLink Quick Plasmid kits, Mini-, Midi- or Maxi-Prep (Invitrogen) or Endo-free Plasmid Maxi Kit (Qiagen). Air-dried plasmid DNA was resuspended in TE buffer or endotoxin free TE buffer.

TE buffer: 10 mM Tris-HCl pH 7.4 (Sigma-Aldrich), 1 mM EDTA (Sigma-Aldrich).

\subsubsection{Determination of DNA concentration}

Absorption measurement at 260 and $280 \mathrm{~nm}$ wavelength by a UltraSpec 3100pro (Amersham) was used to determine the concentration of DNA in aqueous solutions with the formula dsDNA concentration $=\mathrm{OD}_{260}$ X $50 \mu \mathrm{g} / \mathrm{mL}$ X dilution factor. Solutions with $\mathrm{OD}_{260} / \mathrm{OD}_{280}$ ratio within the range 1.8-2.0 were treated as pure preparations, relatively free from protein and RNA contaminants.

\subsubsection{DNA Sequencing}

Sequence analysis of DNA was performed by DNA Core Facility of MPIem using Applied Biosystems 373 DNA Sequencer.

\subsubsection{DNA digestion with endonucleases}

Digestion of DNA prior to further subcloning steps was carried out with a specific restriction endonuclease and appropriate buffer following the New England Biolabs (NEB) manual. For molecular cloning, the digestion reaction was performed using $20 \mathrm{U}$ of restriction endonuclease per $\mu$ g plasmid DNA for 3 hours at a temperature required for a given enzyme.

\subsubsection{DNA ligation}

For ligation reactions, digested destination plasmid and digested DNA insert with compatible ends were mixed at 1:5,3:3, and 5:1 ratios, each reaction then supplemented with T4 DNA ligase (NEB), its buffer, and molecular biology (MB) grade water $\left(\mathrm{MB}-\mathrm{H}_{2} \mathrm{O}\right)$ to 
the final volume of $20 \mu \mathrm{L}$. The ligation reaction was carried out in ice bucket/water, which allowed for little temperature increments from $4^{\circ} \mathrm{C}$ to room temperature for $16-20$ hours.

\subsubsection{DNA precipitation method}

$20 \mu \mathrm{L}$ DNA solution was supplemented with $2 \mu \mathrm{L}$ of $3 \mathrm{M}$ potassium acetate (SigmaAldrich), and $44 \mu \mathrm{L}$ of ice-cold ethanol, and incubated at $-80^{\circ} \mathrm{C}$ for 30 minutes. After centrifugation at $16 \mathrm{krpm}$ for 30 minutes, DNA pellet was washed with $70 \%$ ethanol, air dried, and resuspended in $5 \mu \mathrm{L}$ of TE buffer.

\subsubsection{Isolation of plasmid DNA with phenol/chloroform}

Pellets of E. coli transformed with appropriate plasmids and grown for $16-20$ hours at $37^{\circ} \mathrm{C}$ were resuspended in $300 \mu \mathrm{L}$ buffer $\mathrm{P} 1$, and $300 \mu \mathrm{L}$ of buffer P2 was added to the bacterial slurry. Tube was inverted five times and incubated at room temperature for 5 minutes, upon which $300 \mu \mathrm{L}$ of buffer $\mathrm{P} 3$ at $4^{\circ} \mathrm{C}$ was added. Mixture was incubated on ice for 5 minutes, centrifuged at $4^{\circ} \mathrm{C}$ and $14 \mathrm{krpm}$ for 10 minutes, and supernatant was transferred to $500 \mu \mathrm{L}$ of PCA. Mixture was then centrifuged at $14 \mathrm{krpm}$ for 5 minutes, and $800 \mu \mathrm{L}$ of the aqueous phase was transferred to $560 \mu \mathrm{L}$ of isopropanol (Merck), mixed by inversion, and spun at 14 krpm for 15 minutes at $4^{\circ} \mathrm{C}$. DNA pellet was subsequently washed with $70 \%$ ethanol, air dried, and resuspended in $20 \mu \mathrm{L}$ of TE buffer.

Buffer P1: 8\% (w/v) sucrose (Merck), 5\% (v/v) Triton X-100 (Roche), $50 \mathrm{mM}$ Tris-HCl, pH 8.0, $10 \mathrm{mM}$ EDTA, $100 \mu \mathrm{g} / \mu \mathrm{L}$ RNaseA (Invitrogen).

Buffer P2: $200 \mathrm{mM} \mathrm{NaOH}$ (Merck), 1\% (w/v) SDS (Gerbu).

Buffer P3: 3.0 M potassium acetate, $\mathrm{pH}$ 5.5.

PCA: Phenol/Chloroform/Isoamyl alcohol 50/49/1 (Roche). 


\subsubsection{Isolation of genomic DNA from mouse tail biopsies}

Genomic DNA for the purpose of genotyping was isolated with Genomic DNA Isolation Kit for Tissue and Cells (Nexttec) according to manufacturer's protocol.

\subsubsection{Agarose gel electrophoresis}

Digested DNA, isolated RNA or PCR products, were subjected to electrophoresis in 1-2.5\% agarose (UltraPure, Invitrogen) gel containing 0.1\% GelRed (Biotium). Negatively charged DNA fragments were separated in TBE buffer at $100 \mathrm{~V}$ and then visualized by UV-light (Intas). The appropriate DNA size was estimated with Gene Ruler DNA Ladder Mix (Fermentas), included on the gel.

TBE buffer: $50 \mathrm{mM}$ Tris-HCl (Sigma-Aldrich), $50 \mathrm{mM}$ boric acid (Sigma-Aldrich), $2 \mathrm{mM}$ EDTA (Sigma-Aldrich), pH 8.0.

DNA Loading Dye: $10 \mathrm{mM}$ Tris- $\mathrm{HCl} \mathrm{pH}$ 7.6, 0.03\% (w/v) bromophenol blue (SigmaAldrich), 0.03\% (w/v) xylene cyanol FF (Sigma-Aldrich), 60\% (v/v) glycerol (Merck), 60 mM EDTA.

\subsubsection{Agarose gel extraction}

DNA of interest was excised with a surgical scalpel from the agarose gel and purified with PureLink Gel Extraction Kit (Invitrogen), following manufacturer's protocol.

\subsubsection{Polymerase Chain Reaction (PCR)}

DNA amplification in vitro was performed in PCR [205]. The reaction included template double stranded DNA, dNTPs (GE Healthcare), oligonucleotide primers, DNA polymerase, and the supplied buffer. High-fidelity $P f u$ polymerase was used for cloning applications and Red-Taq DNA polymerase was applied for genotyping or diagnostic purposes. All reactions were carried out on Peltier Thermal Cycler PTC-225 (MJ Research, Bio-Rad) with the following conditions:

Step 1: $94^{\circ} \mathrm{C}$ for 2 minutes,

Step 2: $94^{\circ} \mathrm{C}$ for 30 seconds, 
Step 3: annealing temperature for 30 seconds,

Step 4: $72^{\circ} \mathrm{C}$ for extension time (500 bp per $1 \mathrm{~kb}$ of DNA) (30 cycles from Step 2 to 4 )

Step 5: $72^{\circ} \mathrm{C}$ for 10 minutes.

Annealing temperature was always $5^{\circ} \mathrm{C}$ lower than the mean of estimated melting temperatures of primers in reaction.

\subsubsection{Long-Range PCR}

Amplification of long genomic DNA sequences (i.e. for validation of correct recombination of targeting vector to genomic DNA, Fig. 26E) was performed using Pfu-AD polymerase (kindly provide by Steffan Frey, MPIbpc) in Phusion-HF buffer (NEB) in the presence of 1 M betaine (Sigma-Aldrich), and oligonucleotides 29127 and 29156 with the following parameters:

Step 1: $99^{\circ} \mathrm{C}$ for 3 minutes,

Step 2: $99^{\circ} \mathrm{C}$ for 30 seconds,

Step 3: $60^{\circ} \mathrm{C}$ for 30 seconds,

Step 4: $72^{\circ} \mathrm{C}$ for 90 seconds (30 cycles from Step 2 to 4 )

Step 5: $72^{\circ} \mathrm{C}$ for 2 minutes.

\subsubsection{3'loxP diagnosis}

Multiplex PCR to detect 3'loxP site (Fig. 26F) was performed with standard parameters using oligonucleotide primers: 29244, 29245, 28441.

\subsubsection{Mutant mouse genotyping}

Genotyping for all mutant mouse lines was performed in DNA Core Facility (MPIem, Göttingen) by PCR using oligonucleotide primers listed below (wt, wild type allele; Cre, knock-in allele with Cre; ko, knockout allele; floxed, allele with loxP site). PCR for SRY locus was performed to assess the gender. 
Table 7. Genotyping summary of mouse lines used in this study.

\begin{tabular}{|c|c|c|}
\hline Mouse line & Primers & PCR product size \\
\hline Emxl-Cre & $8546 / 25252 / 25253$ & $\begin{array}{l}\text { wt }-234 b p \\
\text { Cre }-321 b p\end{array}$ \\
\hline NEXI-Cre & $2409 / 18637 / 18638$ & $\begin{array}{l}\text { wt }-236 b p \\
\text { Cre }-356 b p\end{array}$ \\
\hline$m i R-140$ & $28745 / 28746$ & $\begin{array}{l}\text { wt }-233 \\
\text { ko }-256 \text { bp (After }[201])\end{array}$ \\
\hline$W w p 1^{\mathrm{f} / \mathrm{f}} ; W w p 2^{\mathrm{f} / \mathrm{f}}$ & $10908 / 10909 / 12812 / 12809$ & $\begin{array}{l}\text { Wwp1 wt }-182 b p \\
\text { Wwp1 floxed }-304 b p \\
\text { Wwp2 wt }-349 b p \\
\text { Wwp2 floxed }-368 b p\end{array}$ \\
\hline$N e d d 4-2^{f / \mathrm{f}}$ & $12804 / 12806$ & $\begin{array}{l}\mathrm{wt}-224 \mathrm{bp} \\
\text { floxed }-322 \mathrm{bp}\end{array}$ \\
\hline$U b e 3 b^{\mathrm{f} / \mathrm{f}}$ & $29244 / 29245 / 28441$ & $\begin{array}{l}\text { wt }-197 \mathrm{bp} \\
\text { floxed }-345 \mathrm{bp}\end{array}$ \\
\hline$S R Y$ & $28742 / 28741$ & $\begin{array}{l}\text { male }-150 \mathrm{bp} \\
\text { female }- \text { no band (After } \\
[206])\end{array}$ \\
\hline
\end{tabular}

All genotyping PCR were performed with the following parameters:

Step 1: $96^{\circ} \mathrm{C}$ for 3 minutes,

Step 2: $94^{\circ} \mathrm{C}$ for 30 seconds,

Step 3: $64^{\circ} \mathrm{C}$ for 1 minute,

Step 4: $72^{\circ} \mathrm{C}$ for 1 minute (32 cycles from Step2 to 4 ),

Step 5: $72^{\circ} \mathrm{C}$ for 7 minutes.

\subsubsection{TOPO-TA subcloning}

Subcloning of PCR products was performed with TOPO-TA cloning kits (Invitrogen) in pCR2.1-TOPO or pCRII-TOPO vectors according to manufacturer's protocol. 


\subsubsection{Cloning strategies for constructs generated in this study}

\section{pCR2.1-Wwp2/miR-140}

Intronic sequence of $W w p 2$ gene was amplified by PCR using primers 25939/25940, and subcloned to pCR2.1-TOPO. The constructed plasmid was used for further cloning (pCIG2XmiR-140), and for RNA-probe synthesis used in in situ hybridization.

\section{pcDNA-3.2-GW/EmGFP-miR-140}

To generate pre-miR-140, oligos 25831/25832 were mixed at the ratio 1:1, and incubated at $95^{\circ} \mathrm{C}$ for 10 minutes. The thermoblock was then switched off, and the duplex was let anneal for 10 hours. Next, precursor sequence of miR-140 was cloned into pcDNA-3.2GW/EmGFP following manufacturer's protocol (Invitrogen).

\section{pCAG-CTR-Sponge}

CTR sponge was synthetized as a tandem of 16 DNA repeats: 5'AAGTTTTCAGTTTGCTAACA-3' by Life Technologies. The 5' site was additionally equipped with restriction sites for XhoI, BglII, and BspEI restriction nuclease, and 3' was with EcoRI, SalI, and KpnI. In the downstream of the 5' restriction sites, three stop codons were added. Synthesized double strand DNA was digested with XhoI, and SalI, and shuttled to pRaichu-205X, opened with the corresponding restriction enzymes. pRaichu-205X was constructed by Dr. Michiyuki Matsuda's group from pCAGGS vector originally developed by Dr. Jun-ichi Miyazaki, Osaka University Medical School.

\section{pCAG-miR-140-3p-Sponge}

miR-140-3p sponge was synthetized as a tandem of 16 DNA repeats: 5' AACTGGTTCCGAACTGTGGTA-3', and shuttled to pCAG-205X with the same combination of restriction enzymes as for construction of CTR sponge.

\section{pCAG-miR-140-5p-Sponge}

miR-140-5p sponge was synthetized as a tandem of 16 DNA repeats: 5'CTAGCATAGGAGCCAACCACTG-3', and shuttled to pCAG-205X with the same combination of restriction enzymes as for construction of CTR sponge. 
pCIG-2XmiR-140

Precursor sequence of miR-140 (pri-miR-140) in pCR2.1-Wwp2/miR-140 were amplified by PCR with primer pairs of 26082/26084, and 26083/26084. PCR products were subcloned into pCR2.1-TOPO to verify the sequence. Next, both vectors were digested with a pair of restriction enzymes, $\mathrm{XhoI} / \mathrm{SacI}$ or $\mathrm{SacI} / \mathrm{EcoRI}$, to clone a tandem repeat of pri-miR-140 to pCIG plasmid opened with XhoI/EcoRI.

\section{pLKO.1-Venus-sh-mSOX9}

For usage in cell culture experiments and in utero electroporation, $\mathrm{pCX}:$ :myrVenus was digested with BamHI/KpnI, and the Venus expression cassette was cloned into pLKO-shmSox9-2 opened with the same restriction enzymes.

\section{pCR2.1-HECT-Nedd4-2}

DNA sequence encoding for Nedd4-2 HECT domain was amplified by PCR using $27879 / 27880$ primers and P0 cortex cDNA library generated by reverse transcription of total RNA isolated from wild type P0 cortex, subcloned into pCR2.1-TOPO, and used for RNA probe synthesis.

\section{pCR2.1-SB-3'Ube3b}

To generate Southern blot probe for validation of the correct recombination of Ube $3 b$ targeting vector, primers 27678/27679 were used for PCR using DNA isolated from wild type ES cells as a template. Amplified DNA was subcloned into pCR2.1-TOPO.

\section{pCR2.1-HECT-Ube3b}

To generate a template for in situ hybridization probe, primers 27883/27884 were used in PCR to amplify cDNA encoding for Ube3b HECT domain using P0 cortex cDNA library as a template. Amplified DNA was subcloned into pCR2.1-TOPO

\section{pCR2.1-Ube3b (WT, full length)}

cDNA NM_054093.2 encoding full length Ube3b (1070 aa) was amplified with primers 26326/26327, introducing 5'EcoRI and 3'XhoI restriction sites using pYX-Asc-Ube3b as a template. Resulting DNA was then subcloned into pCR2.1-TOPO. 


\section{pCAG-myc-Ube $3 b$}

pCR2.1-Ube3b was digested with EcoRI and XhoI, and ligated with pCAG-myc-1 opened with the same restriction enzymes.

\section{pCR2.1-Ube3b C1038S}

Primers 26703/26704 were used for site-directed mutagenesis to generate pCR2.1-Ube3b $\mathrm{C} / \mathrm{S}$ introducing point $\mathrm{C} 1038 \mathrm{~S}$ mutation into pCR2.1-Ube3b WT.

\section{pCAG-myc-Ube3b C1038S}

pCR2.1-Ube3b C1038S was digested with EcoRI and XhoI, and ligated to pCAG-myc-1 opened with the same restriction enzymes.

\section{pCR2.1-IQ-Ube3b}

Primers 26326/26395 were used on pCR2.1-Ube3b wt template for PCR amplification of 729 bp long DNA fragment encoding for 243 amino acid-long N-terminal fragment of Ube3b including the IQ motif. Amplified DNA was then subcloned into pCR2.1-TOPO, and that plasmid was used to generate pGBD-IQ-Ube3b for in Yeast Two-Hybrid screening.

\section{pCAG-myc-IQ-Ube3b}

pCR2.1-IQ-Ube3b was digested with EcoRI and XhoI, and ligated into pCAG-myc-1 opened with the same restriction enzymes.

\subsubsection{Isolation of RNA}

All tools used for RNA work was thoroughly cleaned with 70\% ethanol (Sigma) and rinsed with RNA-Zap (Thermo Fisher) before the beginning of the procedure. Brain tissue was isolated from mice and flash-frozen in liquid nitrogen until the purification procedure. The tissue was homogenized in appropriate volume of Trizol [207] (Thermo Fisher) - $1 \mathrm{~mL}$ for embryonic cortex, 2 mL per adult cortex - with Ultra Turrax homogenizer (IKA Labtechnik) for 1 minute, and incubated at room temperature for 5 minutes. For every $1 \mathrm{~mL}$ of homogenate, $200 \mu \mathrm{L}$ of chloroform (Merck) was added, mixed well using vortex (Bender and Hobein), incubated at room temperature for 2-3 minutes, and centrifuged 15 minutes at $13 \mathrm{krpm}$. The aqueous phase was transferred to new tubes (Eppendorf), mixed with one volume of $70 \%$ ethanol (Sigma-Aldrich), and the solution was applied onto RNeasy columns 
(RNeasy Mini Kit, Qiagen). For purification of miRNA fraction, miRNeasy purification Kit (Qiagen) was used. Further steps were performed according to the manufacturer's protocols. RNA was eluted from the silica membranes using $30 \mu \mathrm{L}$ of $\mathrm{MB}-\mathrm{H}_{2} \mathrm{O}$.

\subsubsection{Quality validation of RNA preparation}

After purification of RNA, the quality was verified by agarose gel electrophoresis, and quality check and concentration estimation was performed using 2100 Bioanalyzer (Agilent).

\subsubsection{First-Strand cDNA synthesis}

Reverse transcription of purified RNA was carried out with SuperScript III First-Strand Synthesis System (Invitrogen) following manufacturer's manual. For each reaction, $3 \mu \mathrm{g}$ of total RNA was used in the final volume of $20 \mu \mathrm{L}$. After terminating of the reaction by incubation at $85^{\circ} \mathrm{C}$ for 5 minutes, the mixture was cooled on ice for 5 minutes, and $1 \mu \mathrm{L}$ of RNaseH (Invitrogen) was added. Reaction was held for 20 minutes at $37^{\circ} \mathrm{C}$. Thereafter, 21 $\mu \mathrm{L}$ reaction was supplemented with $63 \mu \mathrm{L}$ of $\mathrm{MB}-\mathrm{H}_{2} \mathrm{O}$, and stored at $-20^{\circ} \mathrm{C}$ until further processing.

\subsubsection{Real Time PCR (RT-PCR) for mRNA detection and quantification}

The RT-PCR was performed in 384 well plates (Roche). For each analyzed gene, master mix including $5 \mu \mathrm{L}$ of SYBR Green (Invitrogen), $0.1 \mu \mathrm{L}$ of each RT-primer, and $1 \mu \mathrm{L}$ MB- $\mathrm{H}_{2} \mathrm{O}$ per reaction was prepared. Next, $4 \mu \mathrm{L}$ of cDNA solution was pipetted to each well followed by $6 \mu \mathrm{L}$ of master mix. Pipetting was performed on ice, and the plate covered from light. The reaction was held on Light Cycler 480 (Roche Applied Science) with the parameters listed below. 
Table 8. The parameters of Real Time PCR in this study.

\begin{tabular}{|c|c|c|c|}
\hline Temperature & Duration & Ramp Rate $\left({ }^{\circ} \mathrm{C} / \mathrm{s}\right)$ & Number of cycles \\
\hline \multicolumn{4}{|l|}{ Pre-incubation } \\
\hline $50^{\circ} \mathrm{C}$ & $2 \min$ & 4.8 & \multirow{2}{*}{1} \\
\hline $95^{\circ} \mathrm{C}$ & $10 \mathrm{~min}$ & 4.8 & \\
\hline \multicolumn{4}{|c|}{ Amplification (Quantification) } \\
\hline $95^{\circ} \mathrm{C}$ & $15 \mathrm{~s}$ & 4.8 & \multirow{2}{*}{45} \\
\hline $60^{\circ} \mathrm{C}$ & $1 \mathrm{~min}$ & 2.5 & \\
\hline \multicolumn{4}{|l|}{ Melting Curve } \\
\hline $95^{\circ} \mathrm{C}$ & $10 \mathrm{sec}$ & 4.8 & \multirow{3}{*}{1 (acquisition per $5^{\circ} \mathrm{C}$ ) } \\
\hline $65^{\circ} \mathrm{C}$ & $1 \mathrm{~min}$ & 2.5 & \\
\hline $95^{\circ} \mathrm{C}$ & Continuous & 0.11 & \\
\hline \multicolumn{4}{|l|}{ Cooling } \\
\hline $40^{\circ} \mathrm{C}$ & $10 \mathrm{sec}$ & 2 & 1 \\
\hline
\end{tabular}

Before each gene analysis by RT-PCR, a standard curve using different concentration of cDNA was plotted, and the input amount of cDNA was calculated, enabling the quantification of mRNA levels in the linear range of the input template. Level of transcripts of each analyzed gene: Wwpl (detected with qRT-PCR primers 32140/32141), Wwp2 (31998/31999), and Sox9 (32142/32143) was then normalized to the average level of mRNA of housekeeping genes: Gapdh (14539/14540), and Oazl (19902/19903). Primers for the reaction were designed with Roche Assay Design Center.

\subsubsection{Detection of miRNA by $q R T-P C R$}

For detection of miRNAs, cDNA was synthetized with miScript II RT system (Qiagen) using $3 \mu \mathrm{g}$ of total RNA. Quantitative Real Time PCR (qRT-PCR) was carried out with miScript (Qiagen) primers for miR-140-3p, miR-140-5p, and RNU6B (miRScript Primer Assay) using miScript SYBR Green PCR Kit (Qiagen). Each step of miRNA detection (including duration and temperatures of RT-PCR) was performed in collaboration with Dr. Xin Zhang (GZMB, Molecular Oncology, Göttingen) according to manufacturer's protocol. Level of each miR-140 strand was normalized to the level of RNU6B. 


\subsubsection{Isolation of the genomic DNA from Embryonic Stem Cells (ES Cells)}

The ES cells were grown in 6-well plates (Greiner) until confluency, carefully washed with PBS, and incubated with $1 \mathrm{~mL}$ of lysis buffer for $16-20$ hours at $37^{\circ} \mathrm{C}$. Next, cell lysate was collected to $2 \mathrm{~mL}$ reaction tubes (Eppendorf), and supplemented with $700 \mu \mathrm{L}$ isopropanol (Merck). The mixture was inverted several times, and centrifuged for 15 minutes at $4^{\circ} \mathrm{C}$ at $13 \mathrm{krpm}$. Resulting DNA pellets were subsequently washed with $70 \%$ ethanol (SigmaAldrich), air dried, and resuspended in $100 \mu \mathrm{L}$ of TE Buffer. DNA solutions were kept at $4^{\circ} \mathrm{C}$ until further processing.

PBS buffer: $8 \mathrm{~g} \mathrm{NaCl}, 0.2 \mathrm{~g} \mathrm{KCl}, 1.44 \mathrm{~g} \mathrm{Na}_{2} \mathrm{HPO}_{4}, 0.24 \mathrm{~g} \mathrm{KH}_{2} \mathrm{PO}_{4}$ (all reagents from Merck)

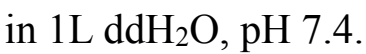

Lysis Buffer: $100 \mathrm{mM}$ Tris, pH 7.5, $5 \mathrm{mM}$ EDTA, 0.5\% (w/v) SDS (Gerbu), $200 \mathrm{mM} \mathrm{NaCl}$, $100 \mu \mathrm{g} / \mathrm{mL}$ Proteinase K (Roche).

\subsubsection{Southern blotting}

The method was modified after previously published work [208]. Restriction digest of $10 \mu \mathrm{g}$ of genomic DNA isolated from ES cells was held using 10U of SapI restriction enzyme (NEB) in $37^{\circ} \mathrm{C}$ for 16 hours. Next, digested genomic DNA was resolved in $0.7 \%$ agarose gel in $500 \mathrm{~mL}$ TAE buffer supplied with $40 \mu \mathrm{L}$ of $1 \%$ ethidium bromide (Carl Roth) at 30V for 16-20 hours. After gel documentation in UV (Intas), agarose gel was incubated in 0.25 $\mathrm{M} \mathrm{HCl}$ (Merck) for 10 minutes, and subsequently in $0.4 \mathrm{M} \mathrm{NaOH}$ (Merck) for 10 minutes. Upward capillary transfer of DNA onto a nylon membrane (Hybond N+, GE Healthcare) was performed in $0.4 \mathrm{M} \mathrm{NaOH}$ for 16-20 hours. Next, the membrane was incubated in 2XSSC until neutralized, and air dried on chromatography paper (Whatman). DNA was further UV-crosslinked with energy density of $1.00 \mathrm{~J} / \mathrm{cm}^{2}$ (FluoLink, Biometra), and the membranes were pre-hybridized in Rapid Hyb-Buffer (GE Healthcare, Amersham) 1 hour at $65^{\circ} \mathrm{C}$. Radioactive probes were synthetized with Prime-It II Random Primer Labeling Kit (Agilent) using $25 \mathrm{ng}$ of EcoRI-digested pCR2.1-SB-3'Ube3b and $\alpha-{ }^{32} \mathrm{P}-$ labeled dCTP (Perkin-Elmer) following manufacturer's protocol. After termination of probe synthesis, the sample was diluted with $\mathrm{MB}-\mathrm{H}_{2} \mathrm{O}$ to final volume of $100 \mu \mathrm{L}$, passed through chromatography columns to remove unincorporated dCTP with Bio-Spin size exclusion column (Bio-Rad). $25 \mu \mathrm{L}$ of the eluate was diluted with $5 \mathrm{~mL}$ of pre-warmed $\left(65^{\circ} \mathrm{C}\right)$ 
hybridization buffer, and incubated with DNA crosslinked on the nylon membrane. Hybridization was held at $65^{\circ} \mathrm{C}$ for 2 hours, followed by extensive washing with $2 \mathrm{XSSC}$ buffer with $0.1 \%$ SDS (sodium dodecyl sulfate, Gerbu) for 15 minutes in room temperature, and subsequently with 1 XSSC buffer with $0.1 \%$ SDS for 7 minutes at $65^{\circ} \mathrm{C}$. Radioactivity of the membrane was then monitored with Geiger-Müller counter, and the washing was continued until the scintillator reported approximately 100 counts. Membrane was then exposed to photographic film (Kodak) for $16-20$ hours at $-80^{\circ} \mathrm{C}$, and developed.

TAE buffer: $40 \mathrm{mM}$ Tris-base (Sigma-Aldrich), $20 \mathrm{mM}$ acetic acid (Sigma-Aldrich), 1mM EDTA (Sigma-Aldrich).

$\underline{\text { 1XSSC buffer: }} 150 \mathrm{mM} \mathrm{NaCl}$ (Merck), $15 \mathrm{mM}$ sodium citrate (Merck), pH 7.0.

\subsubsection{Yeast Two-Hybrid (Y2H) screening}

Screening of Ube3b binding partners using PJ69-4A yeast strain was performed with pGBDIQ-Ube3b bait vector and two cDNA libraries: mouse embryo (in pGAD-GL plasmid) and rat brain (pVP16-3) according to modified previously published protocol [209].

\subsubsection{Media, buffers and stock solutions for Y2H}

Yeast complete and selective media were prepared using $\mathrm{ddH}_{2} \mathrm{O}$, and autoclaved. For preparation of culture plates, $2 \%$ agar $(\mathrm{w} / \mathrm{v})$ was added to media before autoclaving. All recipes are per $1 \mathrm{~L}$, unless specified otherwise.

YPAD

$10 \mathrm{~g}$ yeast extract, $20 \mathrm{~g}$ bacto-peptone, $0.4 \mathrm{~g}$ adenine, $20 \mathrm{~g}$ glucose.

SC-W

$6.7 \mathrm{~g}$ yeast nitrogen base without amino acids, $20 \mathrm{~g}$ glucose, $5 \mathrm{~g}$ casamino acids, $0.2 \mathrm{~g}$ adenine, 0.2 g uracil.

SC-WL

$6.7 \mathrm{~g}$ yeast nitrogen base without amino acids, $20 \mathrm{~g}$ glucose, $50 \mathrm{~mL} 20 \mathrm{X}-\mathrm{WL}$ mix. 
SC-WLH

$6.7 \mathrm{~g}$ yeast nitrogen base without amino acids, $20 \mathrm{~g}$ glucose, $50 \mathrm{~mL} 20 \mathrm{X}-\mathrm{WLH}$ mix.

SC-WLH plus 3AT

$100 \mathrm{~mL}$ of SC-WLH before autoclaving was supplied with $1 \mathrm{~mL}$ of $1 \mathrm{M} 3$-amino-1,2,4triazol (3AT), earlier passed through $0.22 \mu \mathrm{m}$ filter.

SC-WLA

$6.7 \mathrm{~g}$ yeast nitrogen base without amino acids, $20 \mathrm{~g}$ glucose, $50 \mathrm{~mL}$ 20X-WLA mix.

20X Complete Amino Acid Mix

$0.4 \mathrm{~g}$ adenine, uracil, L-tryptophan, histidine $\mathrm{HCl}$, L-arginine, L-methionine, $0.6 \mathrm{~g}$ Ltyrosine, L-isoleucine, lysine $\mathrm{HCl}, 1.2 \mathrm{~g}$ phenylalanine, $0.6 \mathrm{~g}$ leucine.

20X-WL

As above, but without L-tryptophan, and L-leucine.

20X-WLH

As above, but without L-tryptophan, L-leucine, and L-histidine $\mathrm{HCl}$.

20X-WLA

As above, but without L-tryptophan, L-leucine, and adenine.

20X-LA

As above, but without L-leucine, and adenine.

Buffers:

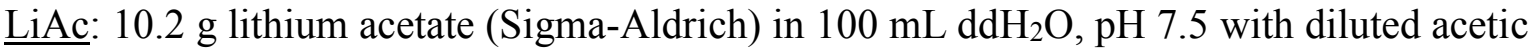
acid (Merck).

Yeast lysis buffer: $1 \%$ (w/v) SDS, $100 \mathrm{mM} \mathrm{NaCl}, 10 \mathrm{mM}$ Tris-HCl pH 8.0, $1 \mathrm{mM}$ EDTA, 2\% (v/v) Trtion X-100.

10XTE: 100 mM Tris-HCl, pH 7.4, 10 mM EDTA. 


\subsubsection{Transformation of DNA into yeast}

PJ69-4A yeast was transformed with the bait vector (pGBD-IQ-Ube3b) with heat shock. 5 $\mathrm{mL}$ of YPAD medium was inoculated with a single yeast colony, and incubated for 16-20 hours at $30^{\circ} \mathrm{C}$. Next, $200 \mu \mathrm{L}$ of the preculture was transferred to $10 \mathrm{~mL}$ of fresh YPAD, and incubated for 3 hours at $30^{\circ} \mathrm{C}$. Yeast were harvested by centrifugation at $3 \mathrm{krpm}$ for 5 minutes at room temperature, resuspended in $1 \mathrm{~mL}$ of $1 \mathrm{XTE} / \mathrm{LiAc}$, pelleted again, and resuspended in $0.5 \mathrm{~mL}$ of $1 \mathrm{XTE} / \mathrm{LiAc} .100 \mu \mathrm{L}$ of cell slurry was supplied with $1 \mu \mathrm{g}$ of plasmid DNA, $5 \mu \mathrm{L}$ of salmon testis DNA (10 mg/mL, Roche), $600 \mu \mathrm{L}$ of 40\%PEG/100mMLiAc/TE (PEG 3350, Sigma-Aldrich), and incubated for 30 minutes at $30^{\circ} \mathrm{C}$ with moderate shaking. Next, to each reaction tube, $70 \mu \mathrm{L}$ DMSO (Roche) was added, the cells were incubated at $42^{\circ} \mathrm{C}$ for 15 minutes, transferred on ice for 1 minute, harvested by centrifugation at $7 \mathrm{krpm}$ for 1 minute, and resuspended in $1 \mathrm{~mL}$ of YPAD to recover for 1 hour at $30^{\circ} \mathrm{C}$. Then, yeast cells were washed with $\mathrm{ddH}_{2} \mathrm{O}$ cultured for $16-20$ hours in appropriate medium, and streaked on selection plate. An aliquot of yeast was streaked on YPAD plate to determine the transformation efficiency.

\subsubsection{Bait construct autoactivity check}

For autoactivity check of the bait construct, yeast cells were transformed as described with the following DNA: pGBD-IQ-Ube3b alone, pGBD-IQ-Ube3b, and pVP-16-3, and pGBDIQ-Ube3b, and pGAD424-HA, and streaked on SC-WL, SC-WLH+3AT, and SC-WLA plates for 3 nights.

\subsubsection{Screening}

Upon validation of no autoactivity of the bait vector, yeast cells were transformed with $1 \mu \mathrm{g}$ of mouse embryo or rat brain cDNA library. After overnight culturing in SC-WL medium, yeast cells were streaked onto SC-WLH+3AT $(10 \mathrm{mM})$ plates, and incubated for 3 nights at $30^{\circ} \mathrm{C}$. Colonies were subsequently transferred onto SC-WLA plate. Yeast colonies formed on SC-WLA agar plate were selected as putative positive clones for further processing. 


\subsubsection{Isolation of yeast DNA}

The positive yeast clones were resuspended in $200 \mu \mathrm{L}$ of $\mathrm{ddH}_{2} \mathrm{O}$, and supplied with $0.3 \mathrm{~g}$ of glass beads, spun down at $3 \mathrm{krpm}$ for 5 minutes, and suspended in $200 \mu \mathrm{L}$ of yeast lysis buffer. Next, the tube was supplied with $200 \mu \mathrm{L}$ of phenol/chloroform mixture, closed, and shaken for 20 minutes at room temperature. Extracted yeast DNA was further collected as an aqueous phase upon centrifugation at $15 \mathrm{krpm}$ for 5 minutes in room temperature, and concentrated by ethanol precipitation method.

\subsubsection{Identification of binding partners}

Yeast DNA was further transformed to E. coli Electro10 or XL1-Blue strain, and seeded on appropriate selection plates. Single colonies were then incubated with $5 \mathrm{~mL}$ of LB medium with selection antibiotics for $16-20$ hours at $37^{\circ} \mathrm{C}$, and plasmid DNA was then isolated with phenol/chloroform DNA isolation method, and sequenced. Binding partners were identified with Basic Local Alignment Search Tool software (BLAST).

\subsubsection{In situ hybridization (ISH)}

For DNA-RNA in situ hybridization [210], digoxigenin-labeled RNA (DIG-RNA) probes were used.

\subsubsection{Probe synthesis}

To determine the orientation of the subcloned DNA sequences, we performed diagnostic PCR with primers specific to T7 and Sp6 promoters of pCR2.1 (89/91) and to the insert. For all RNA-probe templates, Sp6 RNA polymerase yields the generation of DIG-labeled antisense probe, and T7 RNA polymerase generates sense control probe. To generate linearized templates for in-vitro transcription, $7 \mu \mathrm{g}$ pCR2.1 plasmids were linearized by restriction digestion with XhoI for antisense probe synthesis, and with HindIII or BamHI (for Nedd4-2) for sense probe transcription. Digested DNA was subjected to electrophoresis in agarose gel, and isolated with gel extraction kit (Invitrogen). Further the amount of template DNA was estimated by agarose gel electrophoresis using GeneRuler $1 \mathrm{kB}$ ladder (Fermentas) as a standard. Next, DIG-labeled RNA probes were synthetized in an in vitro 
transcription using digoxigenin-11-dUTP (Roche) with SP6/T7 MAXIscript Transcription Kit (Thermo Fisher Scientific) following manufacturer's protocols. The quality of RNA upon in vitro transcription was further analyzed by non-denaturing agarose gel electrophoresis, and ethidium bromide staining.

\subsubsection{Tissue preparation}

For in situ hybridization, C57BL/6N mice at E16 and P21 were used. Embryos, and brains were isolated from deeply anaesthetized mice, washed with ice-cold PBS, flash-frozen in the isopentane bath at -38 to $-40^{\circ} \mathrm{C}$, embedded in OCT Tissue Tec (Sakura), and cryosectioned. Sagittal sections of $20 \mu \mathrm{m}$ for the embryos, and $14 \mu \mathrm{m}$ for the adult brain were collected on Superfrost slides (Thermo Scientific), and air-dried for 10 minutes at room temperature before processing.

\subsubsection{Hybridization}

All solutions for processing of sections before the hybridization were treated with diethylpyrocarbonate (DEPC, Sigma-Aldrich) at a final concentration of $0.1 \%$ or prepared using DEPC-treated $\mathrm{ddH}_{2} \mathrm{O}$.

Sections were subsequently fixed at room temperature with $4 \%(\mathrm{w} / \mathrm{v})$ paraformaldehyde (PFA) for 20 minutes in PB buffer, washed 3 times in PBS, dehydrated with methanol (JT Baker)/PBS series, and incubated in $100 \%$ methanol at $-20^{\circ} \mathrm{C}$ for 20 minutes. Further, sections were rehydrated through reverse methanol/PBS series, beginning with $80 \%$ methanol with $6 \%$ hydrogen peroxide (Merck) in $\mathrm{H}_{2} \mathrm{O}$ for 1 hour. Next, sections were washed with PBST buffer, and treated with $10 \mu \mathrm{g} / \mathrm{mL}$ proteinase $\mathrm{K}$ for 10 minutes. Upon washing 3 times with PBST, sections were post-fixed with 4\% PFA/PB for 5 minutes, again washed 3 times with PBST, rinsed with $0.85 \%$ (w/v) $\mathrm{NaCl}$ solution, incubated in $70 \%$ ethanol prepared in $0.85 \% \mathrm{NaCl}$ solution, and further dehydrated with $95 \%$ ethanol. Sections on slides were air dried for 10 minutes. DIG-probes were denatured by heating at $80^{\circ} \mathrm{C}$ for 5 minutes, and diluted with hybridization buffer to the final concentration of $750 \mathrm{ng} / \mathrm{mL}$. Hybridization was held in chamber humidified with $50 \%$ formamide (SigmaAldrich) $/ 5 \mathrm{XSSC}$ buffer at $37^{\circ} \mathrm{C}$ for 48 hours.

5XSSC Buffer: 750 mM NaCl (Merck), 75 mM sodium citrate (Merck), pH 7.0. 


\subsubsection{Post-hybridization washes and antibody application}

The sections were subsequently washed with $50 \%$ formamide/5XSSC for 3 times, followed by washes in salt gradient (5X-0.2XSSC, each time for 30 minutes), and 3 times in MBST buffer. Next, section underwent blocking with $20 \%$ heat inactivated sheep serum (HISS), (PAA) in MBST for 1 hour at room temperature, followed by incubation with the anti-DIG antibody conjugated with alkaline phosphatase (Roche) diluted 1:1000 in 2\% HISS in MBST at $4{ }^{\circ} \mathrm{C}$ for $16-20$ hours.

\subsubsection{Color Developing}

Next, sections were washed 3 times with MBST, and incubated in the developing buffer (NTMT) for 10 minutes at room temperature. Then, developer solution was added to each slide (NBT/BCIP in NTMT, Roche), the color development was observed and terminated by washing with PBS. Further, coverslips were mounted on slides with Immu-Mount medium (Shandon, Thermo Scientific).

\subsubsection{Image acquisition}

Apochromatic Leica MZ16F steromicroscope was used to visualize the color signal after in situ hybridization,.

4\% paraformaldehyde: 4 g paraformaldehyde (PFA) was added to $40 \mathrm{~mL}$ of $\mathrm{ddH}_{2} \mathrm{O}$ heated to $50^{\circ} \mathrm{C}$, and supplied with $38 \mu \mathrm{L}$ of $10 \mathrm{M} \mathrm{NaOH}$. The solution was stirred until PFA was completely dissolved, cooled down on ice, filled with $50 \mathrm{~mL}$ PB Buffer, and completed with $\mathrm{ddH}_{2} \mathrm{O}$ up to $100 \mathrm{~mL}$. $\mathrm{pH}$ within the range $7.0-8.0$ was verified using $\mathrm{pH}$-strip indicators (Merck).

PB Buffer: $0.1 \mathrm{M}$ phosphate buffer (sodium phosphate dibasic, sodium phosphate monobasic, $\mathrm{pH} 7.2$, Merck).

PBST Buffer: PBS with 0.1\% (w/v) Tween-20 (Sigma-Aldrich).

Hybridization Buffer: 10 mM Tris-HCl, pH 7.5, 600 mM NaCl, 1 mM EDTA pH 8.0, 0.25\% (w/v) SDS, 10\% (w/v) dextran sulfate (Sigma-Aldrich), Denhardt's 1:50 (Thermo Fisher), $200 \mu \mathrm{g} / \mathrm{mL}$ yeast tRNA (Thermo Fisher), 50\% (v/v) formamide, deionized (Sigma-Aldrich). 
MBST Buffer: $100 \mathrm{mM}$ maleic acid (Sigma-Aldrich), $150 \mathrm{mM} \mathrm{NaCl}, \mathrm{pH}$ 7.5, 0.1\% (w/v) Tween-20.

NTMT Buffer: $100 \mathrm{mM} \mathrm{NaCl}, 100 \mathrm{mM}$ Tris-HCl, pH 9.5, 50 mM MgCl 2 (Sigma-Aldrich), $0.1 \%(\mathrm{w} / \mathrm{v})$ Tween-20.

\subsubsection{LNA-based in situ detection of miRNA}

In situ hybridization to detect mature miRNAs was performed in collaboration with Dr. Tamara Rabe and Prof. Ahmed Mansouri (MPIbpc, Göttingen). Wild-type hippocampal neurons grown on poly-L-lysine-coated coverslips at DIV7 were fixed with 4\% PFA, and washed with PBS. Cells on coverslips were then incubated with acetylation solution, and upon 5 minute-long proteinase $\mathrm{K}(5 \mu \mathrm{g} / \mathrm{mL}$ PBS $)$ treatment underwent pre-hybridization at room temperature for $4-8$ hours, followed by hybridization at $55^{\circ} \mathrm{C}$ for $16-20$ hours. For each coverslip, $1 \mathrm{pM}$ of LNA-DIG labeled probe purchased from Exiqon diluted with hybridization buffer was used. Coverslips were subsequently washed in 5XSSC (pH 4.5) at $60^{\circ} \mathrm{C}$ for 5 minutes, and with $0.2 \mathrm{XSSC}$ at $60^{\circ} \mathrm{C}$ for 1 hour. Coverslips were incubated for 10 minutes at room temperature with solution B1 followed by application of an anti-DIG antibody coupled with alkaline phosphatase diluted 1:2000 in blocking solution. After 1620 hours incubation at $4^{\circ} \mathrm{C}$, coverslips were washed in $\mathrm{B} 3$ solution 3 times for 10 minutes, and Developer solution was applied. Color development was stopped with PBS.

Acetylation solution: $0.015 \%(\mathrm{v} / \mathrm{v})$ Triethanoloamine (Sigma-Aldrich), $0.02 \%(\mathrm{v} / \mathrm{v}) \mathrm{HCl}$ (Merck).

Hybridization solution: 50\% deionized (v/v) formamide, 5XSSC, $5 \mathrm{mM} \mathrm{EDTA,} 0.1 \%(\mathrm{v} / \mathrm{v})$ Tween-20, 0.1\% (w/v) 3-((-3-cholamidopropyl)-dimethyloammonio)-1-propanesulfonate (CHAPS, Biomol), $0.1 \mathrm{mg} / \mathrm{mL}$ heparin (Sigma-Aldrich), $1 \mathrm{mg} / \mathrm{mL}$ yeats tRNA.

Solution B1: 0.1 M Tris-HCl, pH 7.5, 0.15 M NaCl.

Blocking solution: $10 \%(\mathrm{v} / \mathrm{v})$ fetal bovine serum (FBS, Gibco), 0.5\% (v/v) Tween-20 in solution B1.

Soultion B3: $0.1 \mathrm{M}$ Tris-HCl, $\mathrm{pH}$ 9.5, 0.1 M NaCl, $50 \mathrm{mM} \mathrm{MgCl}, 0.1$ \% (v/v) Tween-20, 20 $\mu \mathrm{M}$ levamisole-HCl (Sigma Aldrich) passed through $0.45 \mu \mathrm{m}$ filter (Nalgene).

Developer solution: $10 \mu \mathrm{M}$ levamisole-HCl, 0.02\% (w/v) NBT/BCIP in solution B3. 


\subsection{Biochemical experiments}

\subsubsection{Protein concentration measurements}

Protein concentration was estimated with BCA kit (Thermo, Pierce) following manufacturer's protocol The absorbance of protein complexes was measured with Plate Reader (Bio-Rad). Protein concentration in sample was calculated based on the absorbance value for BSA standards (Thermo, Pierce).

\subsubsection{Sodium dodecyl sulfate polyacrylamide gel electrophoresis (SDS-PAGE)}

Proteins were separated under denaturing conditions using SDS-PAGE [211]. Protein samples were dissolved in Sample buffer [212], and incubated at $65^{\circ} \mathrm{C}$ for 15 minutes, loaded on two-layered acrylamide gel, and subjected to electrophoresis with Running buffer (Bio$\mathrm{Rad}$ ) at $90 \mathrm{~V}$ for the stacking gel, and $120 \mathrm{~V}$ for the separating gel. Protein gel, made of stacking, and separating gels was casted according to Bio-Rad Protean II system.

Sample buffer: $10 \%$ (v/v) glycerol, $50 \mathrm{mM}$ Tris-HCl, pH6.8, 2\% (w/v) SDS, 2 mM EDTA, $10 \mathrm{mM}$ DTT, 0.05\% (w/v) Bromophenol blue.

Stacking gel: $5 \%(\mathrm{v} / \mathrm{v})$ acrylamide/N,N'-methylene-bis-acrylamide (35.5:1) solution (National Diagnostics), $125 \mathrm{mM}$ Tris-HCl, $\mathrm{pH}$ 6.8, 9.1\% (w/v) SDS, 0.05\% (w/v) ammonium persulfate (APS, Sigma-Aldrich), $0.005 \% \quad(\mathrm{v} / \mathrm{v}) \quad$ N,N,N',N'tetramethylethylenediamine (TEMED, Serva).

Resolving gel: 8-15\% (v/v) acrylamide/N,N'-methylene-bis-acrylamide (35.5:1) solution, $325 \mathrm{mM}$ Tris-HCl, 0.1\% (w/v) SDS, 0.05\% (w/v) APS, 0.005\% (v/v) TEMED.

Running buffer: $25 \mathrm{mM}$ Tris-HCl, $250 \mathrm{mM}$ glycine (Sigma-Aldrich), 0.1\% (w/v) SDS, pH 8.8 .

\subsubsection{Western blotting}

Separated proteins were electrophoretically transferred onto a nitrocellulose membrane (Amersham Protran, $0.2 \mu \mathrm{m}$ NC, GE Healthcare) in transfer buffer under a constant current of $80 \mathrm{~mA}$ for 10 hours using a tank blotting unit (Hoefer, TE22) [213]. After transfer, membrane was rinsed with $\mathrm{ddH}_{2} \mathrm{O}$ to remove gel debris, and stained with MEM Code 
(Thermo Scientific) kit to visualize separated proteins. After destaining, membrane was rinsed with TBS buffer, and subjected to blocking with blocking buffer for 30 minutes at room temperature with moderate shaking, incubated with the primary antibody diluted in blocking buffer (diluted according to Table 5) for 3 hours at room temperature, and washed 3 times with TBS-T for 15 minutes. Next, the membrane was incubated with secondary antibodies conjugated with horseradish peroxidase (HRP) or fluorophores diluted in blocking buffer according to Table 6 for 1 hour. After subsequent washing (3 times, 15 minutes), the signal on the membrane was developed with chemiluminescence detection system (ECL) on films (Amersham Hyperfilm ECL, GE Healthcare) or detected with Odyssey CLx Infrared Imaging System (LI-COR).

Transfer buffer: $25 \mathrm{mM}$ Tris-base, $190 \mathrm{mM}$ glycine, 20\% (w/v) methanol.

TBS: 10 mM Tris-HCl, $150 \mathrm{mM} \mathrm{NaCl,} \mathrm{pH} \mathrm{7.5.}$

TBS-T: TBS, $0.1 \%$ Tween-20.

Blocking buffer: 5\% (w/v) skimmed milk (Frema), 0.1\% (v/v) Tween-20, TBS.

Washing buffer: TBS, $0.1 \%$ (v/v) Tween-20.

\subsubsection{Affinity binding assay with recombinant proteins}

Binding assay between myc-tagged N-terminus of Ube $3 b$ (IQ-Ube3b) an its putative binding substrates was performed with affinity chromatography by Hiroshi Kawabe. Briefly, GSTfused recombinant pray proteins fragments identified in Yeast Two-Hybrid screenings were purified from E. coli BL21-Rosetta strain using pGEX-GST system (GE Healthcare). $40 \mu \mathrm{g}$ of each GST-fused proteins was immobilized on $50 \mu \mathrm{L}$ of glutathione Sepharose 4B beads (GE Healthcare). After subsequent washing with binding buffer (five bed volume), $500 \mu \mathrm{L}$ of the extract of Human Embryonic Kidney 293 (HEK293) cell expressing myc-IQ-Ube3b was loaded on the GSH-beads. Next, beads were washed with $750 \mu \mathrm{L}$ of wash buffer, and GST-peptides were eluted with elution buffer containing reduced glutathione (SigmaAldrich).

Binding buffer: 20 mM Tris-HCl, pH 7.4, 150 mM NaCl, 1 mM EDTA, 1\% (w/v) CHAPS. Wash buffer: 50 mM Tris-HCl, pH 7.4, 300 mM NaCl, 1 mM EDTA, 1\% (w/v) CHAPS. 
Elution buffer: $40 \mathrm{mM} \mathrm{GSH}, 50 \mathrm{mM}$ Tris-HCl, $\mathrm{pH}$ 7.4, $300 \mathrm{mM} \mathrm{NaCl}, 1 \mathrm{mM}$ EDTA, 1\% (w/v) CHAPS.

All buffers above were supplemented with $0.2 \mathrm{mM} \mathrm{PMSF}, 1 \mu \mathrm{g} / \mathrm{mL}$ aprotinin, and $0.5 \mu \mathrm{g} / \mathrm{mL}$ leupeptin.

\subsubsection{Subcellular fractionation of mouse brains}

Subcellular fractionation in sucrose gradients of the brain tissue was performed in collaboration with Bekir Altas according to published protocols [214,215] with slight modifications. All described procedures were performed at $0-4^{\circ} \mathrm{C}$. Immediately after decapitation, mouse cortex was isolated in Solution A. Cortices from one mouse were homogenized in $3 \mathrm{~mL}$ of Solution A supplemented with $0.2 \mathrm{mM} \mathrm{PMSF}, 1 \mu \mathrm{g} / \mathrm{mL}$ aprotinin, and $0.5 \mu \mathrm{g} / \mathrm{mL}$ leupeptin using Teflon-glass homogenizer with 20 strokes at $1200 \mathrm{rpm}$ (Potter S, Braun). Homogenate (H) was fractionated by centrifugation at $82500 \mathrm{~g}$ for 2 hours in discontinuous sucrose density gradient, comprising of $0.85 \mathrm{M}, 1.0 \mathrm{M}, 1.2 \mathrm{M}$ sucrose solutions. The solution on top of the first interface was collected as supernatant (S), the interface between 0.32 and $0.8 \mathrm{M}$ as the $\mathrm{P} 2 \mathrm{~A}$ fraction (myelin fraction), the one between 0.8 and $1.0 \mathrm{M}$ as the $\mathrm{P} 2 \mathrm{~B}$ fraction (ER/Golgi fraction), the one between 1.0 and $1.2 \mathrm{M}$ as the P2C fraction (synaptosomes), and the pellet as the P2D (mitochondria-enriched fraction) fraction. The P2C fraction was centrifuged at $100 \mathrm{~kg}$ for 20 minutes, and the pellet was resuspended with the hypo-osmotic buffer (6 mM Tris-HCl, $\mathrm{pH} 8.0)$ followed by incubation for 45 minutes to disrupt the synaptosomes. The sample was further centrifuged at $32.8 \mathrm{~kg}$ for 20 minutes to separate the synaptic cytoplasm, and crude synaptic vesicles (SC/CSV) fraction in the supernatant from the crude synaptic membrane (CSM) fraction in the pellet. The CSM fraction was further resuspended with $6 \mathrm{mM}$ Tris- $\mathrm{HCl} \mathrm{pH} 8.0,0.32 \mathrm{M}$ sucrose, and $0.5 \%$ Triton X-100 (Roche), incubated for 15 minutes, and centrifuged at $32.8 \mathrm{~kg}$ for 20 minutes. The pellet contained postsynaptic density fraction (PSD), and the supernatant Triton X-100 extract (TX100-E).

For the purpose of comparative mass spectrometry, CSM fractions were subjected to additional centrifugation in discontinuous sucrose gradient as described above. The interface between 1.0 and 1.2 $\mathrm{M}$ sucrose solutions was collected as the synaptic plasma membrane (SM3) fraction.

Solution A: $0.32 \mathrm{M}$ sucrose (Merck), $1 \mathrm{mM} \mathrm{NaHCO} 3$ (Merck). 


\subsubsection{Comparative label free mass spectrometry}

Purified brain fractions (P2C fractions for identification of miR-140 targets, and SM3 fractions for Ube3b substrates) were subjected to free-label mass spectrometry performed by Dr. Olaf Jahn.

\subsubsection{Proteolytic digestion}

Protein fractions corresponding to $20 \mu \mathrm{g}$ protein were processed according to a filter-aided sample preparation (FASP) protocol [216] modified as described [217]. Briefly, protein samples were lysed and reduced in lysis buffer by shaking for $30 \mathrm{~min}$ at $37^{\circ} \mathrm{C}$, and subsequently loaded on centrifugal filter units (30 kDa MWCO, Millipore). After removal of the detergent by washing twice with wash buffer, proteins were alkylated with $50 \mathrm{mM}$ iodoacetamide in $8 \mathrm{M}$ urea/0.1 M Tris $\mathrm{pH} 8.5$ (20 min at RT), followed by two washes with wash buffer to remove excess reagent. Buffer was exchanged by washing three times with $50 \mathrm{mM}$ ammonium bicarbonate (ABC) containing 10\% acetonitrile. Up to here, all these steps were automated on a liquid-handling workstation equipped with a vacuum manifold (Freedom EVO 150, Tecan) by using an adaptor device constructed in-house, while the following steps were performed by centrifugation to ensure quantitative removal of liquids. After three additional washes with $50 \mathrm{mM} \mathrm{ABC} / 10 \%$ acetonitrile, proteins were digested overnight at $37^{\circ} \mathrm{C}$ with $500 \mathrm{ng}$ trypsin in $40 \mu \mathrm{l}$ of the same buffer. Tryptic peptides were recovered by centrifugation followed by two additional extraction steps with $40 \mu 1$ of $50 \mathrm{mM}$ $\mathrm{ABC}$ and $40 \mu \mathrm{l}$ of $1 \%$ trifluoroacetic acid (TFA), respectively. Aliquots of the combined flow-throughs were spiked with $10 \mathrm{fmol} / \mu \mathrm{l}$ of yeast enolase 1 tryptic digest standard (Waters Corporation) for quantification purposes [218] and directly subjected to LC-MS analysis.

Lysis Buffer: 7 M urea (Sigma-Aldrich), 2 M thiourea (Sigma-Aldrich), 10 mM DTT, 2 \% CHAPS, 0.1 M Tris pH 8.5.

Wash Buffer: $8 \mathrm{M}$ urea, $10 \mathrm{mM}$ DTT, 0.1 M Tris pH 8.5. 


\subsubsection{LC-MS analysis}

Nanoscale reversed-phase UPLC separation of tryptic peptides was performed with a nanoAcquity UPLC system equipped with a Symmetry C18 $5 \mu \mathrm{m}, 180 \mu \mathrm{m} \times 20 \mathrm{~mm}$ trap column, and a HSS T3 C18 $1.8 \mu \mathrm{m}, 75 \mu \mathrm{m} \times 250 \mathrm{~mm}$ analytical column maintained at $45^{\circ} \mathrm{C}$ (Waters Corporation). Injected peptides were trapped for $4 \mathrm{~min}$ at a flow rate of $8 \mu \mathrm{l} / \mathrm{min}$ $0.1 \% \mathrm{TFA}$, and then separated over $120 \mathrm{~min}$ at a flow rate of $300 \mathrm{nl} / \mathrm{min}$ with a gradient comprising two linear steps of 3-35\% mobile phase B in 105 min and 35-60\% mobile phase $\mathrm{B}$ in $15 \mathrm{~min}$, respectively. Mobile phase A was water containing $0.1 \%$ formic acid while mobile phase B was acetonitrile containing $0.1 \%$ formic acid. Mass spectrometric analysis of tryptic peptides was performed using a Synapt G2-S quadrupole time-of-flight mass spectrometer equipped with ion mobility option (Waters Corporation). Positive ions in the mass range $\mathrm{m} / \mathrm{z} 50$ to 2000 were acquired with a typical resolution of at least 20,000 FWHM (full width at half maximum), and data were lock mass corrected post-acquisition. Analyses were performed in the ion mobility-enhanced data-independent acquisition mode with drift time-specific collision energies as described [217,219,220].

\subsubsection{Data analysis and protein quantification}

Continuum LC-MS data were processed for signal detection, peak picking, and isotope and charge state deconvolution using Waters ProteinLynx Global Server version 3.0.2 [221]. For protein identification, a custom database was compiled by adding the sequence information for yeast enolase 1 and porcine trypsin to the UniProtKB/Swiss-Prot mouse proteome, and by appending the reversed sequence of each entry to enable the determination of false discovery rate (FDR). Precursor and fragment ion mass tolerances were automatically determined by PLGS 3.0.2 and were typically below $5 \mathrm{ppm}$ for precursor ions, and below $10 \mathrm{ppm}$ (root mean square) for fragment ions. Carbamidomethylation of cysteine was specified as fixed, and oxidation of methionine as variable modification. One missed trypsin cleavage was allowed. Minimal ion matching requirements were two fragments per peptide, five fragments per protein, and one peptide per protein. The FDR for protein identification was set to $1 \%$ threshold. The freely available software ISOQuant (http://www.isoquant.net) was used for post-identification analysis including retention time alignment, exact mass and retention time (EMRT), and ion mobility clustering, data normalization, isoform/homology filtering, and calculation of absolute in-sample amounts for each detected protein according 
to the TOP3 quantification approach [217,222]. Based on the peptides identified in the first PLGS database search round described above, the stringency for reporting a protein was increased further by considering only peptides with a minimum length of six amino acids, which were identified with scores above or equal to 5.5 in at least two runs. FDR for both peptides, and proteins was set to $1 \%$ threshold, and only proteins reported by two and more peptides were quantified using the TOP3 method.

\subsection{Cell culture}

\subsubsection{Media and solutions}

Papain solution: 20 units of papain (Worthington) were added to $1 \mathrm{~mL}$ of $0.2 \mathrm{mg} / \mathrm{mL}$ cysteine, $1 \mathrm{mM} \mathrm{CaCl}_{2}$, and $0.5 \mathrm{mM}$ EDTA in Dulbecco's Modified Eagle's Medium (DMEM, Gibco, Life Technologies). The solution was then saturated with carbogen - 95\% oxygen, and $5 \%$ carbon dioxide - until clear, and filter-sterilized ( $0.22 \mu \mathrm{m}$, Millipore).

Stop solution: $2.5 \mathrm{mg} / \mathrm{mL}$ bovine serum albumin (BSA, Sigma-Aldrich), $2.5 \mathrm{mg} / \mathrm{mL}$ ovoalbumin (Sigma-Aldrich), 10\% (v/v) FBS in DMEM.

Complete Neurobasal medium: $500 \mathrm{~mL}$ Neurobasal A (Gibco, Life Technologies), $50 \mathrm{~mL}$ FBS (Gibco, Life Technologies), 10 mL B-27 (Gibco, Life Technologies), 5 mL GlutaMAX (Gibco, Life Technologies), $5 \mathrm{~mL}$ penicillin/streptomycin (Life Technologies, Gibco).

HEK cell medium: $500 \mathrm{~mL}$ DMEM, $50 \mathrm{~mL}$ FBS, $5 \mathrm{~mL}$ GlutaMAX, $5 \mathrm{~mL}$ penicillin/streptomycin.

MEF medium: 500 mL KnockOut-DMEM (Gibco, Life Technologies), 75 mL FBS, 6 mL non essential amino acids (Gibco, Life Technologies), 6 mL 200 mM L-glutamine (Gibco, Life Technologies), $\quad 6 \quad \mathrm{~mL} \quad \beta$-mercaptoethanol $\quad$ (Sigma-Aldrich), $3 \mathrm{~mL}$

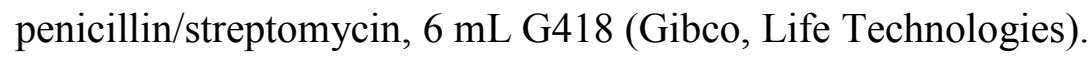

ES cell medium: $500 \mathrm{~mL}$ KnockOut-DMEM, $95 \mathrm{~mL}$ FBS, $6 \mathrm{~mL}$ non essential amino acids, $6 \mathrm{~mL} 200 \mathrm{mM}$ L-glutamine, $6 \mathrm{~mL} \beta$-mercaptoethanol, $3 \mathrm{~mL}$ penicillin/streptomycin, $6 \mathrm{~mL}$ G418, 1000 U Leukemia Inhibitory Factor Lif (Chemicon/Millipore).

Freezing Medium: $30 \mathrm{~mL}$ HEK, MEF or ES cell medium, $10 \mathrm{ml} \mathrm{FBS,} 8 \mathrm{~mL}$ dimethyl sulfoxide (DMSO, Sigma-Aldrich).

RIPA buffer: $20 \mathrm{mM}$ Tris-HCl, pH $7.5\left(4^{\circ} \mathrm{C}\right), 150 \mathrm{mM} \mathrm{NaCl}, 1 \mathrm{mM}$ EDTA, $1 \%$ (v/v) NP40 (Fluka), 1\% (w/v) sodium deoxycholate (Wako Chemicals), PhosphoSTOP (Roche), 10 $\mathrm{mM} \mathrm{NaF}$ (Sigma-Aldrich), 0.2 mM PMSF, $1 \mu \mathrm{g} / \mathrm{mL}$ aprotinin, $0.5 \mu \mathrm{g} / \mathrm{mL}$ leupeptin. 


\subsubsection{Murine primary hippocampal neurons}

\subsubsection{Treatment of coverslips for culturing primary neurons}

Prior to cell culture, coverslips (Menzel-Gläser) were incubated with $1 \mathrm{M} \mathrm{HCl}$ for 16-20 hours, thoroughly rinsed with $\mathrm{dH}_{2} \mathrm{O}$, incubated with $70 \%$ ethanol, again washed with $\mathrm{dH}_{2} \mathrm{O}$, placed in cell culture dishes in sterile conditions (laminar flow hood), and UV-treated for 1 hour. Further, to provide the primary neurons with an adhesion substrate, the coverslips were incubated with poly-L-lysine (Sigma-Aldrich) diluted 1:10 in PBS (Gibco, Life Technologies) for 1 hour at $37^{\circ} \mathrm{C}$. Next, coverslips were rinsed with PBS three times, and incubated with Complete Neurobasal medium at $37^{\circ} \mathrm{C}$ until plating of neurons.

\subsubsection{Neuronal culture}

Brains of P0 mice were dissected on ice-cold Hank's Balanced Salt Solution (HBSS) under stereomicroscope to collect hippocampi in $1 \mathrm{~mL}$ of ice-cold Papain solution. After 1 hour incubation at $37^{\circ} \mathrm{C}$ with gentle shaking, papain solution was carefully replaced by stop solution, and the hippocampi were further incubated at $37^{\circ} \mathrm{C}$ for 15 minutes with moderate agitation. Next, the stop solution was discarded, and hippocampi carefully washed twice with $900 \mu \mathrm{L}$ of pre-warmed $\left(37^{\circ} \mathrm{C}\right)$ Complete Neurobasal medium. Further, the hippocampi were carefully triturated in $200 \mu \mathrm{L}$ Complete Neurobasal medium 10 times using P200 pipette tip. The debris were then let sink for 1 minute, and $150 \mu \mathrm{L}$ the supernatant transferred to $900 \mu \mathrm{L}$ of fresh Complete Neurobasal at $37^{\circ} \mathrm{C}$. After second round of trituration, cells were counted with Naubauer counting chamber, and neurons were seeded in 24 well-plate format. For biochemical experiments, 120000 cells, and for immunocytochemistry, 40000 cells were plated on one coverslip in $1 \mathrm{~mL}$ of Complete Neurobasal medium. For electrophysiological experiments, 4000 cells were plated onto astrocytic microislands in 6 well-plate format. The day of neuronal prep was counted at day in vitro 0 (DIV0). Neurons were cultivated at $37^{\circ} \mathrm{C}$ in the presence of $5 \%$ carbon dioxide in HERA-cell240 (Heraeus) incubator. 


\subsubsection{Calcium phosphate transfection}

Primary hippocampal neurons were transfected at DIV1 with calcium phosphate transfection method. Prior to transfection, the DNA-calcium precipitates were prepared accordingly to published works [223]. For one coverslip of 24 well-plate, $1 \mu \mathrm{g}$ of plasmid DNA was mixed with $3.1 \mu \mathrm{L}$ of $2 \mathrm{M} \mathrm{CaCl}_{2}$, and sterile $\mathrm{H}_{2} \mathrm{O}$ was added till $25 \mu \mathrm{L}$ of total volume. The DNA mix was dropwise added to $25 \mu \mathrm{L}$ of $2 \mathrm{XHBS}, 1 / 8$ of the solution at a time with gentle agitation for 2 seconds after each drop. The solution was then incubated at room temperature in darkness for 30 minutes. Complete Neurobasal medium was replaced with the same volume of Neurobasal A, filtered, and stored at $37^{\circ} \mathrm{C}$. Upon pipetting up, and down for 5 times, $50 \mu \mathrm{L}$ of the DNA-calcium solution was applied on each coverslip, and the neurons were placed at $37^{\circ} \mathrm{C}$ in the incubator until precipitates became visible in the light microscope (approximately 20 minutes). Coverslips were subsequently washed with HBSS equilibrated in $10 \%$ carbon dioxide at $37^{\circ} \mathrm{C}$ until all visible precipitates disappeared. The original complete Neurobasal medium was placed back on the neurons, and cells were further cultivated at $37^{\circ} \mathrm{C} / 5 \% \mathrm{CO}_{2}$.

2XHBS: $274 \mathrm{mM} \mathrm{NaCl}, 10 \mathrm{mM} \mathrm{KCl}, 1.4 \mathrm{mM} \mathrm{Na}_{2} \mathrm{HPO}_{4}, 15 \mathrm{mM}$ glucose, $42 \mathrm{mM}$ HEPES (Sigma-Aldrich, H3375), pH 7.08 adjusted with $10 \mathrm{M} \mathrm{NaOH}$. Buffer was aliquoted, and stored at $-20^{\circ} \mathrm{C}$.

\subsubsection{HEK293FT cell line}

HEK293FT were used for production of lentivirus, and expression of myc-tagged IQ-Ube 3b. Unless stated otherwise, HEK293FT cells were maintained in $6 \mathrm{~cm}$ Petri dishes (Corning) in $10 \mathrm{~mL}$ HEK cell medium at $37^{\circ} \mathrm{C}$ in the presence of $5 \%$ carbon dioxide in HERA-cell 240 (Heraeus) incubator. For passaging, semi-confluent cells were washed with PBS, incubated with $1 \mathrm{~mL}$ of $0.05 \%$ trypsin solution (Gibco, Life Technologies) for 1 minute at $37^{\circ} \mathrm{C}$, and the reaction was stopped by addition of $9 \mathrm{~mL}$ of the fresh medium. HEK293FT cells were split on new Petri dishes. 


\subsubsection{Transfection}

Transfection of HEK293FT was performed at 80-90\% confluency using Lipofectamine2000 (Invitrogen) following manufacturer's instructions with 1:2 DNA/Lipofectamine2000 ratio $(\mu \mathrm{g} / \mu \mathrm{L})$.

\subsubsection{Lentivirus production and infection of primary hippocampal cells}

Viral particles expressing GFP, and GFP together with iCre were produced by Dr. Hiroshi Kawabe. Briefly, HEK293FT cells grown in OPTI-MEM supplemented with 10\% FBS on $15 \mathrm{~cm}$ Petri dish, were co-transfected with $40 \mu \mathrm{g}$ of pFUGW or $40 \mu \mathrm{g}$ of pFUGWiCre with $16 \mu \mathrm{g}$ of packaging plasmid (PACK), and $16 \mu \mathrm{g}$ of envelope plasmid (ENV). Culture medium was exchanged to the HEK cell medium with $2 \%$ FBS upplied with $10 \mathrm{mM}$ sodium butyrate (Gibco, Life Technologies) 4-6 hours after transfection. Culture medium was harvested 48 hours post-transfection, filtered through $45 \mu \mathrm{m}$ filters (Millipore), and applied to AMICON filter system (Millipore) to concentrate viral particles by centrifugation at 3500 $g$ at $4{ }^{\circ} \mathrm{C}$ to approximately $100 \square \mathrm{L}$. The filter was subsequently washed with cold Neurobasal A. The virus stock was further dialyzed against TBS at $4^{\circ} \mathrm{C}$ for $16-20$ hours in a sterilized beaker, aliquoted, and flash-frozen in liquid nitrogen. Viruses were stored in $-80^{\circ} \mathrm{C}$ until used. Upon thawing the aliquot of the virus stock, appropriate volume of the virus stock was added to neuronal medium at DIV1. The virus titer was determined by estimation of GFP expression level in neuronal cultures. At appropriate stage of the culture, infected neurons were washed with PBS four times, and lysed with RIPA buffer at $4^{\circ} \mathrm{C}$. The homogenate was centrifuged for 10 minutes at $14 \mathrm{~kg}$ in cold bench-top microfuge, and supernatant was used in biochemical experiments.

TBS: $20 \mathrm{mM}$ Tris-HCl, $\mathrm{pH} 8.0\left(4^{\circ} \mathrm{C}\right), 150 \mathrm{mM} \mathrm{NaCl}$.

\subsubsection{Mouse Embryonic Fibroblasts (MEF)}

Neomycin-resistant MEFs were purchased from Cell Biolabs (CBA-311) as feeder cells for embryonic stem (ES) cell culture. MEFs were maintained in $15 \mathrm{~cm}$ Petri dishes coated with gelatin $\left(0.1 \%\right.$ gelatin in $\mathrm{ddH}_{2} \mathrm{O}$, Sigma-Aldrich) at $37^{\circ} \mathrm{C}$ in $25 \mathrm{~mL}$ MEF medium in the presence of $5 \%$ carbon dioxide in HERA-cell240 (Heraeus) incubator. At confluency, 
medium was removed, cells were washed with $37^{\circ} \mathrm{C}$-warm PBS, and incubated with $10 \mathrm{~mL}$ MEF medium supplemented with $100 \mu \mathrm{L}$ of mitomycin C (Sigma-Aldrich) per plate for 2.5 hours at $37^{\circ} \mathrm{C}$. Then, medium was removed, cell washed with PBS, trypsinized for 5 minutes with $0.05 \%$ trypsin-EDTA solution (Gibco, Life Technologies) at $37^{\circ} \mathrm{C}$, resuspended with fresh MEF medium, and centrifuged at $1 \mathrm{krpm}$ for 5 minutes at room temperature. Inactivated MEF were then resuspended, Freezing medium was added dropwise to the suspension, and cells were frozen in Cryo Freezing Container (Nalgene) filled with isopropanol at $-80^{\circ} \mathrm{C}$, and seeded prior to demand.

\subsubsection{ES cells}

For generation of Ube3b knockout mice, mutant JM8.F6 ES cells were purchased from EUCOMM Consortium. The L1L2_gt0 cassette was inserted to the genome at position 114389925 (UCSC Genome Bioinformatics) of Chromosome 5 upstream of exon 7 of Ube $3 \mathrm{~b}$ gene. The cassette was composed of an FRT-flanked lacZ/neomycin sequence followed by a 5' loxP site. An additional loxP site is inserted 3' downstream of exon 7 at position 114390710. ES cells were thawn prior to blastocyst injection to minimize the passage number. Cells were seeded on a layer of previously prepared and thawn inactivated MEF, and maintained in ES cell medium at $37^{\circ} \mathrm{C}$ in the presence of $5 \%$ carbon dioxide in HERAcell240 (Heraeus) incubator. Before splitting, cells were supplied with fresh medium for at least 3 hours. Passaging was carried out with 0.25\% trypsin-EDTA (Gibco, Life Technologies), and the reaction was terminated with FBS. Prior to blastocyst injection, MEFs were separated from ES cells by plating cell suspension on fresh gelatinized Petri dish for 30 minutes at $37^{\circ} \mathrm{C}$ allowing MEFs to adhere to the bottom of the dish. Transfer of injected blastocysts to pseudo-pregnant foster female mice was performed in MPIem Animal House Facility by Monika Schindler and Dr. Ursula Fünfschilling.

\subsection{Immunocytochemistry}

Cells cultured on coverslips were fixed with cold $\left(4^{\circ} \mathrm{C}\right) 4 \%$ PFA, 4\% sucrose (Merck) in PB buffer for 20 minutes in room temperature, washed three times with PBS, and incubated with blocking buffer for 30 minutes. Next, cells were incubated with primary antibody diluted with blocking buffer accordingly to Table 5 for $16-20$ hours at $4{ }^{\circ} \mathrm{C}$ with moderate shaking, followed by washing 3 times for 30 minutes with PBS. Further, secondary antibody coupled 
to appropriate fluorophore diluted in blocking buffer was applied for 2 hours at room temperature. After washing 3 times with PBS for 30 minutes, coverslips were briefly rinsed

with $\mathrm{ddH}_{2} \mathrm{O}$, and mounted on Superfrost Plus glass slides (Thermo Scientific) with ImmuMount mounting medium (Shandon, Thermo-Scientific).

Blocking buffer: $5 \%$ goat serum (Gibco, Life Technologies), 0.3\% Triton X-100 (Roche), $0.1 \%$ fish skin gelatin (Sigma-Aldrich) in PBS buffer

\subsection{Histology}

\subsubsection{Perfusion}

Intracardiac perfusion with 4\% PFA in PB buffer was conducted using pump apparatus PASF (IKA Labortechnik) according to the protocols published before [224]. Perfusion was performed on animals older than P3. P0 brains were isolated from the skulls, and immediately immersed in 4\% PFA in PBS for 16-20 hours. Briefly, mice older than P3 were deeply anesthetized by intraperitoneal administration of avertin - 99\% 2,2,2-tribromoethanol (Alta Aesar) dissolved in 2-methylbuthan-2-ol (Sigma) (0.2 mL per mouse of approximately $20 \mathrm{~g}$ ). Mouse was then placed in darkness to reach surgical plane of anesthesia, verified by toe pinch-response. Lateral incision through abdominal wall was followed by separation of the diaphragm from the liver, and diaphragm was further incised together with the rib cage until the full exposure of the pleural cavity. Upon fixation of the sternum with hemostat above the animal head, sterile 25 -gauge winged cannula was introduced to the heart through the posterior end of the left ventricle. Cannula was fixed to the heart with a hemostat. A small incision with iris scissors was performed in the heart's right atrium preventing damage of the descending aorta to create outlet valve. Initial PBS flush was performed at pump speed 12 for 1 minute, and paraformaldehyde solution was then delivered at a constant rate (pump speed 10) for 2 minutes starting with the observation of the first fixation tremors. The brain was further isolated from animal's skull, and incubated with 4\% PFA in PB buffer at $4{ }^{\circ} \mathrm{C}$ for 16-20 hours with moderate agitation. Next, brains were transferred to PBS with $0.01 \%$ sodium azide (Sigma-Aldrich), and kept at $4^{\circ} \mathrm{C}$ until further processing. 


\subsubsection{Cryosectioning}

All histological procedures excluding preparation of tissue for in situ hybridization, included brain sections prepared on Leica CM3050S cryostat. Prior to cryosectioning, brains were incubated for at least 5 hours with 10\% sucrose in PBS, followed by incubation with $30 \%$ sucrose in PBS until the organs reached the bottom of the glass beaker. Next, brains were frozen in -38 to $-40^{\circ} \mathrm{C}$ isopentane (Roth). Coronal cryosections of appropriate thickness were either collected immediately on Superfrost Plus glass slides or in PBS/0.01\% sodium azide solution. Sections of $50 \mu \mathrm{m}$ thickness were collected for spine morphology reconstruction, Bielschowsky Silver Impregnation, and immunohistochemistry, and $100 \mu \mathrm{m}$-thick sections were used for neuronal distribution and polarity classification studies.

\subsubsection{Bielschowsky Silver Impregnation (BSI)}

The staining procedure was performed accordingly to previously published work with slight modifications [225]. Brain sections of $50 \mu \mathrm{m}$ thickness from PFA-perfused mice were collected on Superfrost Plus glass slides, and air-dried for at least 4 hours in room temperature. Slides were then immersed in pre-warmed $\left(40^{\circ} \mathrm{C}\right) 10 \%(\mathrm{w} / \mathrm{v})$ silver nitrate (Sigma-Aldrich) solution for 15 minutes until sections turned light brown. Next, slides were placed in $\mathrm{ddH}_{2} \mathrm{O}$, and washed 3 times. Concentrated ammonium hydroxide was then added dropwise to silver nitrate solution until solution turned cloudy. Slides were incubated with so prepared ammonium silver solution for 1 hour at $40^{\circ} \mathrm{C}$ until color development. Slides were subsequently placed in developer working solution for 1 minute, and reaction was stopped by a quick dip of the slides in $1 \%$ ammonium hydroxide. Slides were then washed three times with $\mathrm{ddH}_{2} \mathrm{O}$, placed in $5 \%$ (w/v) sodium thiosulfate (Sigma-Aldrich), washed three times with $\mathrm{ddH}_{2} \mathrm{O}$, dehydrated, and cleared through $95 \%$ ethanol, absolute ethanol (Sigma-Aldrich), and xylene $(\mathrm{CVH})$, followed by mounting with Entellan New resinous medium (Electron Microscopy Sciences).

Developer stock solution: $20 \mathrm{~mL}$ 37-40\% formaldehyde (Merck), $0.5 \mathrm{~g}$ citric acid, trisodium

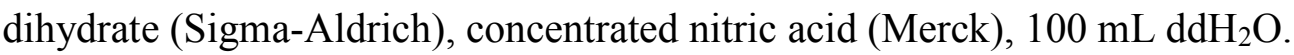

Developer working solution: 8 drops of developer stock solution, 8 drops of concentrated

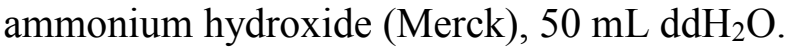




\subsubsection{Immunohistochemistry}

Brain sections of PFA-perfused mice were washed with PBS three times at room temperature, and incubated for 5 minutes with moderate agitation with $1 \mathrm{mg} / \mathrm{mL}$ sodium borohydride (Sigma-Aldrich) solution in PBS. Subsequently, the sections were thoroughly washed with PBS, and incubated with blocking solution for 1 hour at room temperature. Further, sections were incubated with the primary antibody, and DAPI diluted in blocking buffer accordingly to Table 5 for $16-20$ hours at $4{ }^{\circ} \mathrm{C}$, washed in PBS three times for 30 minutes, and incubated with secondary antibody coupled to a fluorophore of choice, according to Table 6. Next, sections were incubated with PBS for 30 minutes three times, and mounted with cover glass (Menzel-Gläser) and mounting medium (Immu-Mount).

Blocking solution: $5 \%$ goat serum, $0.5 \%(\mathrm{v} / \mathrm{v})$ Triton X-100, PBS.

\subsection{In utero electroporation (IUE)}

\subsubsection{Glass capillary preparation}

Micropipettes for IUE were prepared from $1.5-1.8$ X $100 \mathrm{~mm}$ borosilicate glass capillaries (Kimble and Chase) using HEKA PIP5 temperature controlled pipette puller. To ensure the appropriate tip diameter, the filament was heated to $1000^{\circ} \mathrm{C}$, and pulling of glass capillary was controlled manually, allowing for slow expansion of heated glass.

\subsubsection{Plasmid DNA for IUE}

Plasmid DNA was prepared with EndoFree Plasmid Maxi Kit (Qiagen). Prior to IUE, DNA was diluted in endotoxin-free TE buffer, and mixed with $0.1 \%$ Fast Green FCF (SigmaAldrich). Final concentration of DNA used for transfecting cortical progenitors was 200-500 $\mathrm{ng} / \mu \mathrm{L}$, and for electroporation of hippocampal progenitors $-1.2 \mu \mathrm{g} / \mu \mathrm{L}$. Before injection to embryonic brain, the tip of the microcapillary was broken with fine forceps to enable liquid flow. 


\subsubsection{Mice used for IUE}

Table below summarizes the genetic background, and origin of animals used for IUE in this study.

Table 9. Characteristics of mice used for IUE described in this study.

\begin{tabular}{|c|c|c|c|}
\hline \multicolumn{2}{|l|}{ Experiment } & \multirow{2}{*}{$\begin{array}{l}\text { Genetic } \\
\text { background }\end{array}$} & \multirow{2}{*}{ Origin } \\
\hline Mouse line & Plasmid injected & & \\
\hline Wild-type & $\begin{array}{l}\text { pLKO.1-Venus-sh-mWwp1, } \\
\text { pLKO.1-Venus-sh-mWwp2, } \\
\text { pLKO.1-Venus-Scramble }\end{array}$ & $\begin{array}{l}\text { C57BL/6N X } \\
\text { FVBN }\end{array}$ & $\mathrm{N} / \mathrm{A}$ \\
\hline$W w p 1^{\mathrm{f} / \mathrm{f}} ; W w p 2^{\mathrm{f} / \mathrm{f}}$ & $\begin{array}{l}\text { pFUGW, } \\
\text { pFUGWiCre, } \\
\text { pCX::myrVenus }\end{array}$ & $\begin{array}{l}\text { C57BL/6N X } \\
12901 a\end{array}$ & $\begin{array}{l}\text { Generated by } \\
\text { Dr. Hiroshi } \\
\text { Kawabe }\end{array}$ \\
\hline $\operatorname{miR}-140^{-/-}$ & $\begin{array}{l}\text { pFUGW, } \\
\text { pFUGWiCre, } \\
\text { pCX::myrVenus }\end{array}$ & $\begin{array}{l}\text { C57BL/6N X } \\
\text { V.6 }\end{array}$ & $\begin{array}{l}\text { Provided by } \\
\text { Prof. Tatsuya } \\
\text { Kobayashi }\end{array}$ \\
\hline Wild-type & $\begin{array}{l}\text { pCAG-CTR-Sponge, } \\
\text { pCAG-miR-140-3p-Sponge }\end{array}$ & $\mathrm{C} 57 \mathrm{BL} / 6 \mathrm{~N}$ & N/A \\
\hline Wild-type & $\begin{array}{l}\text { pLKO.1-Venus-Scramble, } \\
\text { pLKO.1-Venus-sh-mSox9 }\end{array}$ & $\mathrm{C} 57 \mathrm{BL} / 6 \mathrm{~N}$ & N/A \\
\hline $\operatorname{Sox} 9^{\mathrm{f} / \mathrm{f}}$ & pCAG-EGFP, pCAG-Cre & $\begin{array}{l}\text { C57BL/6N X } \\
129 / \mathrm{SvEv}\end{array}$ & $\begin{array}{l}\text { Provided by } \\
\text { Dr. Mikio } \\
\text { Hoshino }\end{array}$ \\
\hline $\begin{array}{l}W w p 1^{\mathrm{f} / \mathrm{f}} ; W w p 2^{\mathrm{f} / \mathrm{f}} \\
N E X 1-\mathrm{Cre}^{+/-}\end{array}$ & pFUGW, pCX::myrVenus & $\mathrm{C} 57 \mathrm{BL} / 6 \mathrm{~N}$ & $\begin{array}{l}\text { Generated by } \\
\text { Dr. Hiroshi } \\
\text { Kawabe }\end{array}$ \\
\hline $\begin{array}{l}\text { Nedd4-2 } 2^{\mathrm{f} / \mathrm{f}} ; \mathrm{NEX} 1- \\
\mathrm{Cre}^{+/-}\end{array}$ & pFUGW, pCX::myrVenus & $\mathrm{C} 57 \mathrm{BL} / 6 \mathrm{~N}$ & $\begin{array}{l}\text { Generated by } \\
\text { Dr. Hiroshi } \\
\text { Kawabe }\end{array}$ \\
\hline$U b e 3 b^{\mathrm{f} / \mathrm{f}} ; E m x 1-\mathrm{Cre}^{+/-}$ & pFUGW, pCX::myrVenus & $\mathrm{C} 57 \mathrm{BL} / 6 \mathrm{~N}$ & $\begin{array}{l}\text { Generated in } \\
\text { this study }\end{array}$ \\
\hline$U b e 3 b^{\mathrm{f} / \mathrm{f}}$ & $\begin{array}{l}\text { pFUGW, } \\
\text { pFUGWiCre, } \\
\text { pCX::myrVenus }\end{array}$ & $\mathrm{C} 57 \mathrm{BL} / 6 \mathrm{~N}$ & $\begin{array}{l}\text { Generated in } \\
\text { this study }\end{array}$ \\
\hline
\end{tabular}

N/A - not applicable 


\subsubsection{Procedure}

E14.5 mouse embryos were subjected to IUE according to permit number 33.19-42502-0413/1052, and previously published work [72,226]. Pregnant mouse was deeply anesthetized with isolfurane (Ecuphar)/oxygen diffusor (Plexx, HNG6), and placed on warm heating pad $\left(32^{\circ} \mathrm{C}\right)$. Before surgery, the heart rate or the breathing frequency was noted, and the toe pinch-reflex enabled to assess the surgical plane. A layer of Vidisic gel (Bausch and Lomb) was applied onto each cornea to prevent their damage. To ensure post-operative analgesia, $3 \mathrm{mg}$ buprenorphine (Temgesic) was administered subcutaneously. Abdominal wall was thoroughly washed with $70 \%$ ethanol, and $50 \mathrm{mg} / \mathrm{mL}$ povidon-iodine (VetSept). Incision of about $2 \mathrm{~cm}$ in the abdominal cavity was made to expose entire uterus with embryos, which were subsequently moistened with warm PBS supplied with penicillin $2000 \mathrm{U} / \mathrm{mL}$, and 2 $\mathrm{mg} / \mathrm{mL}$ streptomycin (Gibco). Micropipette with the DNA solution was carefully injected to the lateral ventricle of the embryonic brain, and exhausted by expiratory pressure through a pneumatic pump (PV820). The forceps-type electrode (CUY650P1, NepaGene) was placed around the embryo head accordingly to the targeted brain region [73], and electric current was applied with ElectroSquare Porator (ECM830BTX Harvard Apparatus). For electroporation of the cortical progenitors, 5 pulses of $34 \mathrm{~V}$ were delivered, and for transfection of the hippocampal progenitors -8 pulses, each of $32 \mathrm{~V}$, each pulse of $50 \mathrm{~ms}$ duration with $950 \mathrm{~ms}$ interval. After pulsing, embryo head was thoroughly washed with warm PBS with antibiotics. Next, the uterus with embryos was carefully placed back to the abdominal cavity, and the body wall was closed with absorbable surgical sutures (Safil, Aesculap; 4/0, 28"; HR17), and the skin with 9 mm Autoclips (Clay Adam). The animals were kept on the warm pad until back to consciousness. The mouse was monitored postoperatively until deceased.

\subsubsection{Brain processing after in utero electroporation.}

Brains were isolated from skulls of P0 mice, and incubated with 4\% PFA in PB buffer at $4{ }^{\circ} \mathrm{C}$ for $16-20$ hours, washed with PBS, subjected to sucrose gradient $(10 \%-30 \% \mathrm{w} / \mathrm{v}$ sucrose in PBS), and sectioned with cryostat (100 $\mu \mathrm{m}$ thickness). For P10 and P21 mice, the intracardiac perfusion was performed prior to isolation of the brains. After histological processing, brain sections were subjected to immunohistochemistry. 
Imaging of brain coronal cross sections after in utero electroporation was performed at -1.06 $\mathrm{mm}$ to $-2.06 \mathrm{~mm}$ from Bregma for P10 brains [227], and at the level of anterior commissure for P0 brains.

\subsection{Image acquisition, analysis and statistics}

\subsubsection{Quantitative Western blotting}

Quantification of protein levels was performed with ImageStudioLite (Li-Cor) software using scans of films after exposure to ECL chemiluminescence or data acquired by Odyssey cLX. The bands representing respective proteins were manually outlined, and the signal intensity was measured. Signal represents the sum of the individual pixel intensity values for a selected shape subtracted by the average intensity value of the pixels in the background, and the total number of pixels within the region of interest. Protein level was expressed relative to the signal for $\beta$-actin detected in respective lane or total protein as measured by the integral of MEM Code Staining intensity measured with Tracing tool of ImageJ software [228]. For statistical analysis of difference between two groups, t-test was used, and p-value of less than 0.05 was considered as significant.

\subsubsection{Axon counting assays in vitro}

For quantification of number of axons projected by single primary hippocampal neurons, number of cells projecting 0,1 or multiple axons were counted. Axons were defined as processes with prominent SMI312 staining, and no MAP2 signals. Cells were imaged, and scored by a bling observer. Images of transfected neurons were acquired with AxioImager Z.1 (Carl Zeiss), 40X objective and water immersion, and analyzed with AxioVision software. Each quantification represents data from at least two different primary cultures. For statistical analyses of axon acquisition, $\chi^{2}$ test was employed, and for comparison of frequencies in contingency table format, Fisher's exact test was employed.

\subsubsection{Quantification of distribution of cortical neurons and polarity classification}

Z-stacks of $100 \mu \mathrm{m}$-thick mouse cortices after in utero electroporation were acquired with Leica Sp2 confocal microscope with 10X objective (for P0 cortices - zoom 1.5, for P10 
without any digital zoom). Maximum projections of confocal stacks were divided into 5 bins of identical dimensions. Number of transfected neurons in each bin was manually counted with Cell Counter plug-in of ImageJ software, and the number of neurons in each bin expressed relative to the total number of transfected nerve cells. Based on previously published works [229-234], neurons were manually scored based on their morphologies into respective classes (Bipolar with two processes emerging from opposite poles of the soma parallel to the axis perpendicular to pia or Non-bipolar for P0 cortices, and No Polarity, Pyramidal, Multipolar, and Misoriented according to criteria in Table 10 ), and frequencies of neurons of each class within a bin were quantified using Cell Counter plug-in of ImageJ software. Tracing of representative neurons were performed manually after thresholding of binarized images. For statistical analyses, two-way ANOVA with appropriate post-hoc test indicated in the figure legend was used.

Table 10. Classification of neuronal polarities after in utero electroporation.

\begin{tabular}{|l|l|l|}
\hline Polarity class & $\begin{array}{l}\text { Number of apical } \\
\text { dendrites }\end{array}$ & Comment \\
\hline No Polarity & 0 & $\begin{array}{l}\text { No apparent dendrite with prominent apical } \\
\text { shaft }\end{array}$ \\
\hline Pyramidal & 1 & $\begin{array}{l}\text { One prominent apical dendrite oriented } \\
\text { perpendicular to the pial surface }\end{array}$ \\
\hline Multipolar & Multiple & Multiple prominent dendrites \\
\hline Misoriented & 1 & $\begin{array}{l}\text { Apical dendrite forms an angle larger than } \\
15^{\circ} \text { with an axis perpendicular to the pial } \\
\text { surface }\end{array}$ \\
\hline
\end{tabular}

Note: Over the course of this study, we analyzed distribution and morphology of cortical pyramidal neurons in wild type mice as well as in several mouse lines at different stages of development. It appears that the cortical distribution of layer II/III pyramidal neurons differs along anteroposterior and dorsoventral axis of the brain. It is therefore critical that coronal brain sections through cortex from the control and experimental group are imaged at morphologically equivalent positions for the quantification of neuronal distribution.

\subsubsection{Spine morphometrics with STED nanoscopy}

Spine morphology was quantified with ImageJ software using images acquired with STED microscope in collaboration with Dr. Katrin Willig (MPIem, Göttingen) as described before 
[235]. Spines were manually counted using Cell Counter plug-in of ImageJ software, and classified into morphology classed: stubby, filopodia, thin, mushroom, and bifurcated using morphological criteria described before [236]. Spine length was measured as the distance from spine emergence on the dendrite until the head tip. The head size was measured as the diameter of spine head. For statistical analyses, t-test for comparison of two groups, and oneway ANOVA with appropriate post-hoc test for analysis of spine classification were conducted.

\subsubsection{Quantification of axon length and dendritic tree complexity}

Axon length was measured using confocal images of neurons acquired by Leica Sp2 with $20 \mathrm{X}$ objective and ImageJ software. Number of primary branches were counted manually using Cell Counter plug-in. To measure the complexity of dendritic arbor, images of primary hippocampal neurons were acquired with AxioImager Z.1 (Carl Zeiss), using 40X objective and water immersion. Next, we applied Sholl analysis with $7.5 \mu \mathrm{m}$ interval between Sholl circles with median span type on tresholded and binarized images using ImageJ. For quantification of dendritic tree complexity, we quantified a total number of crossings with Sholl circles made by neurites of an individual neuron. For statistical analyzes, t-test was used to compare two independent samples, and one way ANOVA with respective post-hoc tests for comparison of more than two groups.

\subsubsection{Confocal imaging of immunostained brain sections and analysis}

For imaging of the overview of Cux1, Sox9, Ctip2, and NeuN immunostaining, Leica Sp2 confocal microscope with 10X objective was used. Cux1-positive neuronal nuclei expressing miR-140-3p-Sponge were manually counted using Cell Counter plug-in in ImageJ software. Cortical thickness of $U b e 3 b^{\mathrm{f} / \mathrm{f}}$ and $U b e 3 b^{\mathrm{f} / \mathrm{f}} ; E m x 1-\mathrm{Cre}^{+/-}$was measured as the distance from the pia to the end of layer VI on coronal cross section. Tresholded binarized images after Watershed segmentation algorithm using ImageJ were used for quantification of number of neurons. NeuN-positive punctae more than $50 \mu \mathrm{m}^{2}$ in size were counted as somata. For statistical analysis to compare two groups, t-test was employed.

To acquire representative images of primary branches of CA1 apical dendrites of neurons expressing GFP and myrVenus, and for quantitative imaging of Sox 9 signal from Sox $9^{\mathrm{f} / \mathrm{f}}$ neurons expressing GFP, or GFP and iCre, Leica Sp5 microscope with 40X objective with 
confocal scanning Zoom 2.5 was used. Quantitative imaging was performed using ImageJ measure tool on manually selected somata.

\subsubsection{Light and epifluorescence microscopy}

Apochromatic fluorescence stereomicroscope Leica MZ16F was used to acquire images of Bielschowsky Silver Impregnation and immunostainings against GFAP, Iba1, and MAC3.

\subsection{Electrophysiology}

Electrophysiological characterization of Ube $3 \mathrm{~b}$ knockout in neurons using autaptic culture system was performed by Dr. Silvia Ripamonti. Gamma-oscillations from CA3 hippocampal neurons were recorded in collaboration with Dr. Silvia Ripamonti and Dr. Matthieu Hammer. Both experiments were supervised by Dr. Jeong Seop Rhee (Molecular Neurobiology, MPIem).

\subsubsection{Autaptic neurons}

Briefly, autaptic cultured neurons (9-14DIV) were whole-cell voltage-clamped at $-70 \mathrm{mV}$ with the amplifier EPSC10 (HEKA) under the control of the Patchmaster 2 program (HEKA) [237]. All acquired traces were analyzed using AxoGraph X (AxoGraph Scientific). Recordings were performed with a patch-pipette solution and the extracellular solution (BASE $+\mathrm{Ca}^{2+}+\mathrm{Mg}^{2+}$ ). EPSCs and IPSCs were evoked by depolarizing the cell from -70 to $0 \mathrm{mV}$ at a frequency of $0.2 \mathrm{~Hz}$. The ready releasable pool (RRP) size was measured after $6 \mathrm{~s}$ application of $0.5 \mathrm{M}$ hypertonic sucrose solution. The vesicular release probability, Pvr, was calculated as a ratio of the charge transferred during an action potential inducedPSC divided by the charge during sucrose application. Short-term plasticity was measured by recording PSC during $10 \mathrm{~Hz}$ stimulation. Miniature EPSC (mEPSC) and IPSC (mIPSC) were recorded in the presence of $300 \mathrm{nM}$ tetrodotoxin (TTX, Tocris Bioscience). Surface expression of glutamate, and GABA receptors was measured by application of $100 \mu \mathrm{M}$ glutamic acid (Sigma) or $3 \mu \mathrm{M}$ GABA (Sigma), respectively. 
Patch-pipette solution: (all reagents from Sigma-Aldrich): 138/16.8 mM potassium gluconate/Hepes pH 7.4 at 310 mOsm, 10 mM NaCl, 1 mM MgCl $2,0.25$ mM EGTA, 4 mM ATP- $\mathrm{Mg}^{2+}, 0.3 \mathrm{mM}$ GTP-Na ${ }^{+}$.

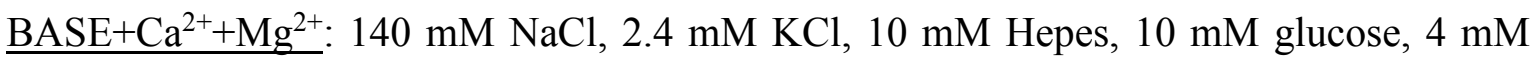
$\mathrm{CaCl}_{2}$, and $4 \mathrm{mM} \mathrm{MgCl} 2$ at $320 \mathrm{mOsml} /$ liter, $\mathrm{pH} 7.3$.

\subsubsection{Gamma-oscillations}

Gamma oscillations were recorded in $U b e 3 b^{\mathrm{f} / \mathrm{f}}$ and $U b e 3 b^{\mathrm{f} / \mathrm{f}} ; E m x 1-\mathrm{Cre}^{+/-}$mice at P15-23. Transverse hippocampal sections (300 $\mu \mathrm{m}$ thickness) were prepared from age-matched littermates as described previously [238] using a Leica VT1200S vibratome. Brain isolation and slice preparation were performed in sucrose-based slicing solution at $4{ }^{\circ} \mathrm{C}$ in the presence of carbogen. After sectioning, slices were placed in a holding chamber containing artificial cerebrospinal fluid (ACSF) and let recover for 15 min before recording. Gamma-oscillations were recorded in CA3 field of hippocampus, where the peak power of kainate-induced gamma-oscillations is the highest [239]. Interface recording chamber (BSCBU Base Unit with the BSC-HT Haas Top, Harvard Apparatus) was used during recordings. Slices were steadily perfused with $\mathrm{ACSF}$, and $33^{\circ} \mathrm{C}$ temperature was maintained. Extracellular recording electrode filled with ACSF was inserted into CA3 pyramidal cell layer, and extracellular field potential were recorded for more than 20 min before the addition of $50 \mathrm{nM}$ or $100 \mathrm{nM}$ kainate. Baseline (20 min), and oscillatory activity (30 min) were measured using a 700B amplifier (Axon Instruments, Molecular Devices), low pass Bessel filtered at $3 \mathrm{KHz}$, and digitized by the Digidata 1440A data acquisition system (Axon Instruments, Molecular Devices). All traces were analyzed using Axograph X software (AxoGraph Scientific). Power spectra were calculated upon Fourier transforms of 10-min epochs (last 10 min of each recording) of recorded field activity. The baseline power spectrum was subtracted from the power spectrum obtained during kainate application. The dominant frequency within $25-$ $45 \mathrm{~Hz}$ was used to quantify the peak frequency. The power was calculated as the area under the respective peak in the power spectrum.

Sucrose-based slicing solution: $230 \mathrm{mM}$ sucrose, $26 \mathrm{mM} \mathrm{NaHCO}_{3}, 2 \mathrm{mM} \mathrm{KCl}, 1 \mathrm{mM}$ $\mathrm{KH}_{2} \mathrm{PO}_{4}, 2 \mathrm{mM} \mathrm{MgCl} 2,10 \mathrm{mM}$ D-glucose, $0.5 \mathrm{mM} \mathrm{CaCl}_{2}$.

ACSF: $120 \mathrm{mM} \mathrm{NaCl}, 26 \mathrm{mM} \mathrm{NaHCO}_{3}, 1 \mathrm{mM} \mathrm{KH}_{2} \mathrm{PO}_{4}, 2 \mathrm{mM} \mathrm{KCl}, 1 \mathrm{mM} \mathrm{MgCl} 2,10 \mathrm{mM}$ D-glucose, $2 \mathrm{mM} \mathrm{CaCl}_{2}$. 


\subsection{Behavioral testing}

Behavioral analysis of Ube3b conditional knockout mice (cKO) was performed by Prof. Ekrem Dere and Prof. Hannelore Ehrenreich (Clinical Neuroscience, MPIem, Göttingen).

\subsubsection{Animals and housing conditions}

For behavioral testing, mice were housed in groups of 5 in standard cages, with food, and water available ad libitum. Animals were kept under a $12 \mathrm{~h}$ light-dark cycle with lights on at 7:00 AM, and an ambient temperature of $20-22^{\circ} \mathrm{C}$.

\subsubsection{Behavioral phenotyping}

All behavioral experiments were performed by a blind observer. Experiments were conducted during the light phase of the day (between 8:00 AM and 5:00 PM). Male $U_{b e} 3 b^{\mathrm{f} / \mathrm{f}} ; \operatorname{Emx} 1-\mathrm{Cre}^{+/-}$mice $(\mathrm{N}=11)$, and their control Ube3b ${ }^{\mathrm{f} / \mathrm{f}}$ littermates $(\mathrm{N}=8)$ were tested in an extensive behavioral test battery. The behavioral tests were conducted as previously described [240-243]. In this study, we only present the results concerning water-maze, nesting, social interaction in pairs and sociability, and social memory in the tripartite chamber. The age of mice at the beginning of testing was 8 weeks.

\subsubsection{Spatial learning, and memory: Morris water-maze}

Spatial learning and memory were assessed with the water maze test. A large circular tank (1.2 $\mathrm{m}$ in diameter and $0.68 \mathrm{~m}$ in depth) was filled with opaque water to a depth of $0.6 \mathrm{~m}$. An escape platform $(10 \times 10 \mathrm{~cm})$ was submerged $1 \mathrm{~cm}$ below the surface. The swim trajectory of the mouse was recorded by a computer and the video-tracking system VIEWER 2 (Biobserve). The escape latency, swim speed, and path length were measured for each mouse. During the first 2 days, mice were trained to swim towards a visible platform (visible platform task), marked with a $15 \mathrm{~cm}$ high black flag, and placed randomly in different locations across trials (non-spatial training). The extra-maze cues were hidden during these trials. After 2 days of visible platform training, hidden platform training (spatial training) was performed. For 8 days, mice were trained to find a hidden platform (i.e. the flag was removed), located at the center of one of the four quadrants of the pool. The location of the 
platform was fixed throughout testing. Mice had to navigate using extra-maze cues that were placed on the walls of the testing room. Every day, mice performed 4 trials with an intertrial interval of $5 \mathrm{~min}$. For each trial, mice were placed into the pool facing the side wall randomly, and allowed to swim until they found the platform or for a maximum of $90 \mathrm{~s}$. A mouse that failed to find the platform within $90 \mathrm{~s}$ was guided to the platform. The animal then remained on the platform for $20 \mathrm{~s}$ before being removed from the pool. After completion of the hidden platform training, a spatial probe trial was conducted. The platform was removed from the pool, and mice were allowed to swim freely for $90 \mathrm{~s}$.

The preference for the former platform zone was expressed as percent of time spent in the target zone relative to the total time spend in the pool (90 s). Control and cKO mice were also compared regarding the number of entries into the target zone.

\subsubsection{Social behavior}

\subsubsection{Nesting}

The mice were single-housed for $1 \mathrm{~h}$ in the cage with bedding material and nesting towels before lights were turned off. After 2 nights of habituation, nesting towels were replaced by nestlets (pressed cotton squares $\sim 3 \mathrm{~g}$ of weight). Nest building was assessed on the next morning. The remainder/leftover of the nesting material was weighted, and the proficiency of nest building was rated using a scale that ranged from 1-5 with lower scores indicating poor nest building behavior.

\subsubsection{Social interaction in pairs}

Every mouse was first individually habituated to the testing cage $(30 \times 30 \times 30 \mathrm{~cm})$ for $10 \mathrm{~min}$ over 2 consecutive days. Then, 3 pairs of unfamiliar mice of the same genotype were placed in the testing cage for $10 \mathrm{~min}$. The duration of closed contact was recorded by a trained observer who was unaware of mouse genotypes. 


\subsubsection{Sociability and social memory (Tripartite chamber)}

Social preference and memory was tested in the tripartite chamber. The apparatus consisted of a rectangular box that was divided into 3 chambers $(40 \times 20 \times 22 \mathrm{~cm})$. The dividers were made of transparent Plexiglas and had rectangular entries $(35 \times 220 \mathrm{~mm})$. The floor of the box was covered with bedding, exchanged between trials. The test mouse was introduced into the middle chamber, with the entries to the other 2 chambers closed, and allowed to acclimatize for $5 \mathrm{~min}$. Next, a small wire cage $(140 \times 75 \times 60 \mathrm{~mm})$ containing an unfamiliar male $\mathrm{C} 57 \mathrm{BL} / 6 \mathrm{~N}$ mouse of the same age and the similar weight (stranger 1) was placed in one outer chamber. An empty wire cage was positioned in the other outer chamber. The location (outer left or right chamber) of stranger 1 was alternated between trials. After unblocking the entries to the outer chambers the test mouse was allowed to freely move between chambers for $10 \mathrm{~min}$. Time spent in and number of entries into each chamber were recorded by a video tracking system (Viewer2, Biobserve). Each mouse received second and third trials. The second trial was identical to the first, except for the stranger mouse being placed into the other outer chamber to control for possible side bias. On the third trial, the test mouse was presented with the familiar stranger 1 and an unfamiliar stranger 2 . Sociability and social memory indexes were calculated as follows: Sociability index $=($ time investigating stranger $) /(($ time investigating stranger $)+($ time investigating empty cage $)) x 100$; Memory index $=$ (time investigating unfamiliar mouse $) /($ time investigating unfamiliar+familiar mouse)x 100 .

\subsection{Statistical analysis}

All statistics were performed using SPSS v.17 (San Diego, USA) or Prism Graph Pad software. 


\section{RESULTS}

\section{1.miR-140, Wwp1 and Wwp2, and Sox9 as regulators of neuronal development}

\subsubsection{Downregulation of Wwp1 and Wwp2 in primary hippocampal neurons leads to axon acquisition defects}

Although the roles of Wwp1 and Wwp2 HECT-type ubiquitin ligases have been investigated in dividing cells, the function of both ligases in postmitotic neurons remains yet to be unveiled. Interestingly, an ortholog of murine $W w p 1, C$. elegans $w w p-1$, has been functionally correlated to sad-1, an ortholog of mammalian SAD-A and SAD-B kinases, and to syd-2, an ortholog of mammalian Liprin- $\alpha$ [244]. Despite a well-described role in controlling neurotransmitter release at the presynaptic specializations [245], SAD kinases govern proper axon-dendrite polarity acquisition in worm and mouse [233,234,246]. Indeed, former work of Mika Suga [199] in the lab proved that Wwp1 and Wwp2 are indispensable for acquisition of axons and formation of neuronal polarity. To further corroborate her findings and extend our understanding of the molecular context both ligases operate in, we transfected primary hippocampal neurons with a plasmid carrying a scramble sh-RNA (control) or with two plasmids, each carrying an sh-RNA targeting Wwp1 or Wwp2 to downregulate both ligases (Wwp1/2 double knock-down, dKD). All three knock-down constructs contained a myristoilated Venus (myr-Venus) expression cassette that allowed the visualization of cellular morphology. Relatively low efficiency of the calcium phosphate transfection method employed enabled the tracing of neurites projected from a single neuron. Nerve cells were fixed seven days after plating (DIV7). Primary hippocampal neurons, at this stage of culture, display apparent neuronal polarity with a prominent single axon and multiple dendrites. Immunostaining against the marker proteins: neurofilament $M$, recognized by SMI312 antibody, and MAP2 allows the identification of axons and dendrites (Fig. 5A, a, and b). We then quantified numbers of neurons projecting zero, one, or multiple axons. While $71 \%$ of control neurons projected a single axon, only $46 \%$ of analyzed Wwp1/2 dKD neurons did so. Strikingly, $39 \%$ of Wwp1/2 KD neurons specified multiple axons, and $14 \%$ showed a lack of SMI312-positive neurites (Fig. 5B), indicating that Wwp1, and Wwp2 are necessary for proper axon acquisition in primary hippocampal neurons. To ascribe the observed phenotype to the loss of Wwp1 and Wwp2, we biochemically analyzed the effect of sh-RNA on the levels of both ligases in neurons. Consequently, Western blotting 
analysis demonstrated almost complete depletion of Wwp1 (Fig. 5C) and Wwp2 (Fig. 5D) from neurons infected with lentiviruses expressing sh-RNAs against Wwp1 or Wwp2 as compared to those infected with virus carrying scramble sh-RNA.

\section{A}

a. Control
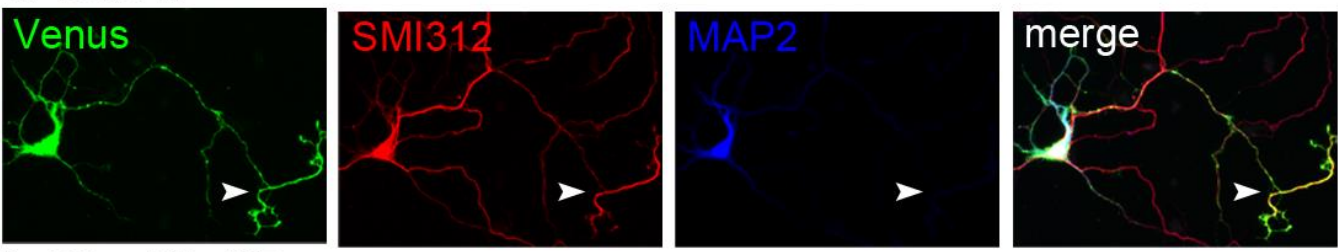

b. Wwp1/2 dKD
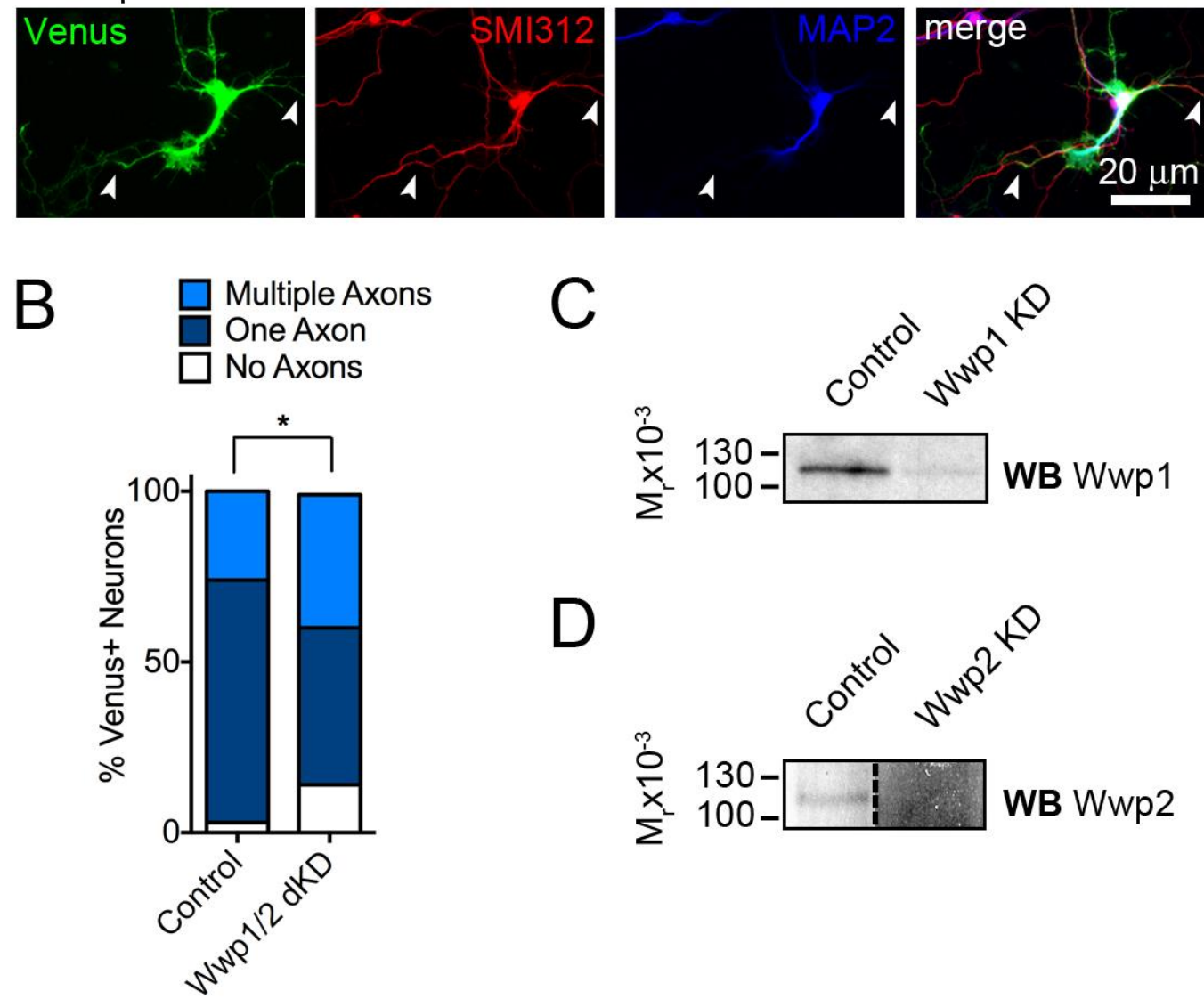

Figure 5. Simultaneous knock-down of Wwp1 and Wwp2 results in defects in axon acquisition in primary cultured neurons. (A) Images of representative primary hippocampal neurons transfected with plasmid encoding scramble sh-RNA as a control (a) or with two plasmids encoding sh-RNAs to knock-down Wwp1 and Wwp2 (b). Neurons were transfected at DIV1 and fixed at DIV7. Arrowheads indicate axons with prominent SMI312 and undetectable MAP2 signals. Scale bar, $20 \mu \mathrm{m}$. (B) Quantification of the number of axons projected from a single neuron. Counted were relative numbers of neurons with 0 , 1 , or multiple axons. Control, $\mathrm{n}=35$ neurons; Wwp $1 / 2 \mathrm{dKD}, \mathrm{n}=56$ neurons; $* \mathrm{p}<0.05$, Chi-square test. (C, and D) Validation of shRNA expression vectors for Wwp1 (C) and Wwp2 (D) by Western blotting. Neurons were infected with lentiviruses expressing non- 
silencing sh-RNA (Control), or sh-RNAs for each E3 ubiquitin ligase (sh-Wwp1 or shWwp2) at DIV1 and neuronal lysates were harvested at DIV7.

\subsubsection{Knock-down of Wwp1 and Wwp2 in cortical progenitors leads to striking neuronal polarity defects and disrupts distribution of neurons in the cortical plate}

Shortly after plating, primary neurons form lamellipodia, which extend as short neurites after $6-12$ hours. Neuronal polarization takes place around 24 hours post-plating, when one of the neurites undergoes rapid outgrowth and acquires axonal fate [47]. This in vitro model of neuronal polarization recapitulates major aspects of neuronal polarity acquisition in vivo. After cell cycle exit through asymmetric cell division, postmitotic cortical neurons undergo transition to multipolar cells, projecting multiple neurites from the soma. One of the neurites emerges in radial direction and becomes a leading process. This facilitates radial translocation of the neuronal cell body alongside radial glial cell processes, which in turn specifies a trailing process located on the opposite pole of the nerve cell. Later on, the leading process becomes the main apical dendritic shaft, and the trailing one extends as an axon [247,248]. Disturbances of axon specification observed upon Wwp $1 / 2$ dKD in primary hippocampal neurons are likely to be paralleled by defects in specification of trailing and leading processes in newborn neurons, and leads to aberrances of neuronal migration, resulting in incorrect positioning of nerve cells throughout the cortical plate. To corroborate this hypothesis, and examine the effects of knock-down of Wwp1 and Wwp2 in vivo, we electroporated the pool of wild type cortical progenitors at E14.5 in utero with control scramble, or Wwp1/2 dKD sh-RNA expression vectors. Brains were sectioned at postnatal day 0 (P0), and postnatal day 10 (P10) followed by immunostaining of Venus to visualize cellular morphology, and study distribution of neurons throughout the cortex. DAPI staining (Fig. 6A, left panels) showed normal cortical layering and morphology, indicating that the gross structure of the brain was not affected by the surgery. We acquired Z-stacks of images of $100 \mu \mathrm{m}$-thick cross sections through a cortex per brain, maximum projected them into one image, and divided the cortical plate into five bins of identical dimensions from bin 1 marking pial surface to bin 5 covering the ventricular zone. Next, we quantified the number of Venus-expressing neurons in each bin. The number of neurons counted was then expressed relative to total number of electroporated nerve cells. Already at P0, a majority of 
neurons expressing control, non-silencing sh-RNA completed migration to the upper layers of the cortical plate, whereas newborn neurons expressing sh-RNAs for Wwp1 and Wwp2 (Wwp1/2 dKD) displayed different distribution in the cortical plate (Fig. 6A, right panels). While most of control neurons completed migration and settled in cortical bin 2 (control, $77.1 \pm 3.3 \%$ ), Wwp $1 / 2 \mathrm{dKD}$ nerve cells displayed rather diffuse distribution in all bins, and only $46.1 \pm 2.6 \%$ of them were found in bin 2 (Fig. 6B). At this stage of development, migrating neurons acquire characteristic bipolar morphology with the leading and the trailing processes, each becoming the dendrite and the axon, respectively. Interestingly, some delayed neurons expressing sh-RNAs against Wwp1 and Wwp2 projected two leading processes or only one extending downwards, towards ventricular zone (Fig. 6C, lower panel, cell 2, and 3 from the right). Hence, we then scored control and Wwp1/2 $\mathrm{dKD}$ neurons as 'Bipolar' or 'Non-Bipolar' based on the number and orientation of their leading and trailing processes. The number of cells of a given class was then expressed relative to total neuron number within a bin. There was significant decrease in the frequency of bipolar cells upon Wwp1/2 dKD as compared to control in lower layers of developing cortical plate (control $100 \%, \mathrm{Wwp} 1 / 2 \mathrm{dKD}-81.7 \pm 3.3 \%$ of neurons in bin 4 were bipolar) (Fig. 6D).

Postmitotic neurons inherit their axon-dendrite polarity from the apical-basal organization of the processes of their neuronal progenitors [249-251]. Hence, defects in polarity acquisition at early developmental stages (i.e. in P0 cortex) are likely to persist during brain development. To validate this hypothesis, we analyzed transfected neurons at P10 as well, and concluded that aberrances in polarity formation are striking and paralleled by diffuse distribution of neurons throughout the cortical layers (Fig. 7A). We then quantified Venusexpressing neurons in each bin of cortex in a similar way as for the P0 brains. Majority of control neurons localized evenly to bin one and two (cortical layers II/III, bin $1-42.6 \pm 8$ $\%$, bin $2-41.0 \pm 3.2 \%$, bin $3-5.6 \pm 2.4 \%$, bin $4-0.8 \pm 1.0 \%$, and bin $5-10.1 \pm 7.1 \%$ ), which is the destination of pyramidal cortical neurons derived from E14/E15 progenitors [252]. Upon Wwp $1 / 2$ dKD, neurons distributed rather evenly throughout the cortex (bin $1-$ $22.2 \pm 5.3 \%$, bin $2-37.3 \pm 4.4 \%$, bin $3-16.9 \pm 3.8 \%$, bin $4-5.3 \pm 2.1 \%$, and bin $5-$ $18.2 \pm 3.3 \%$ ) (Fig. 7B). 

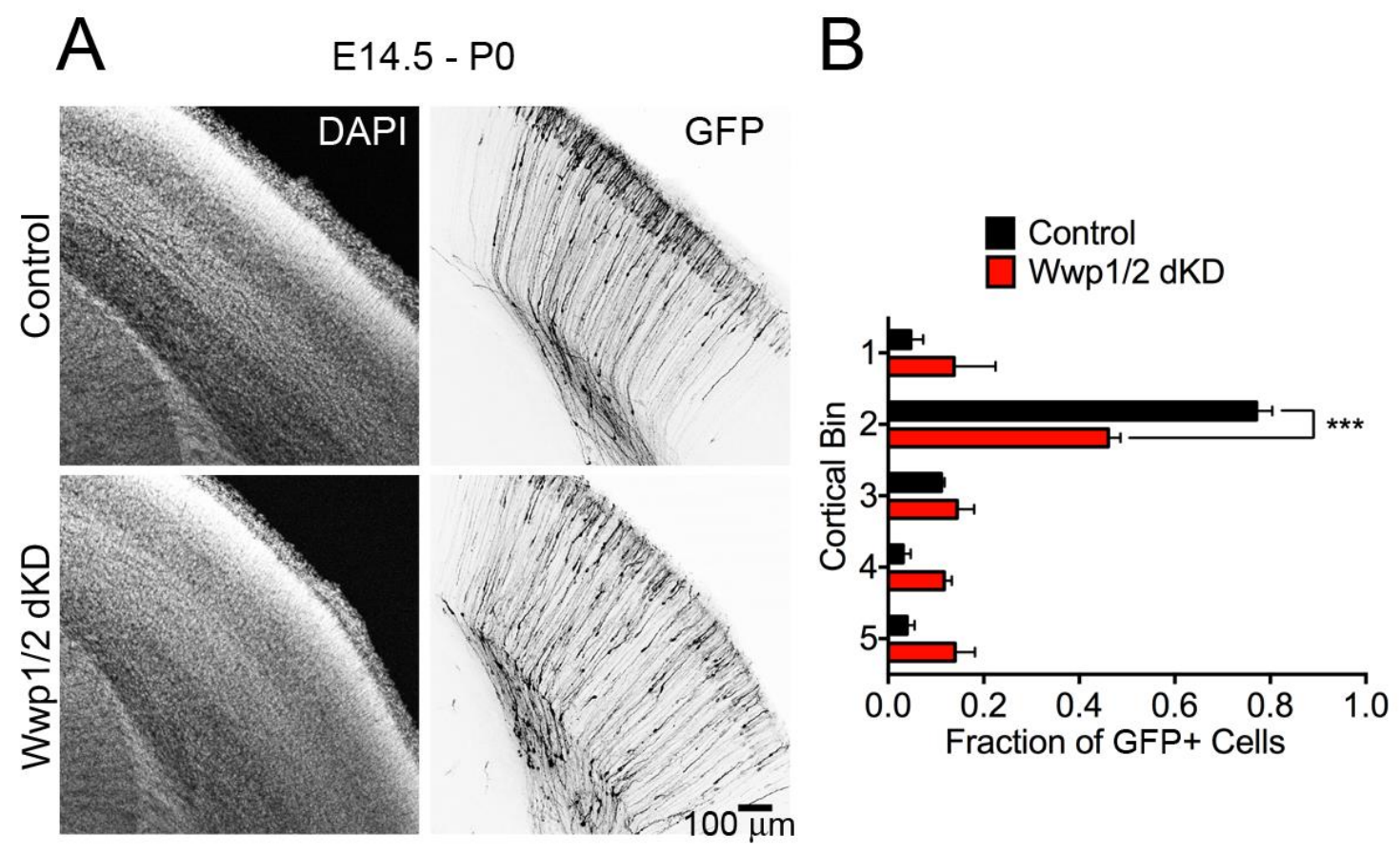

B
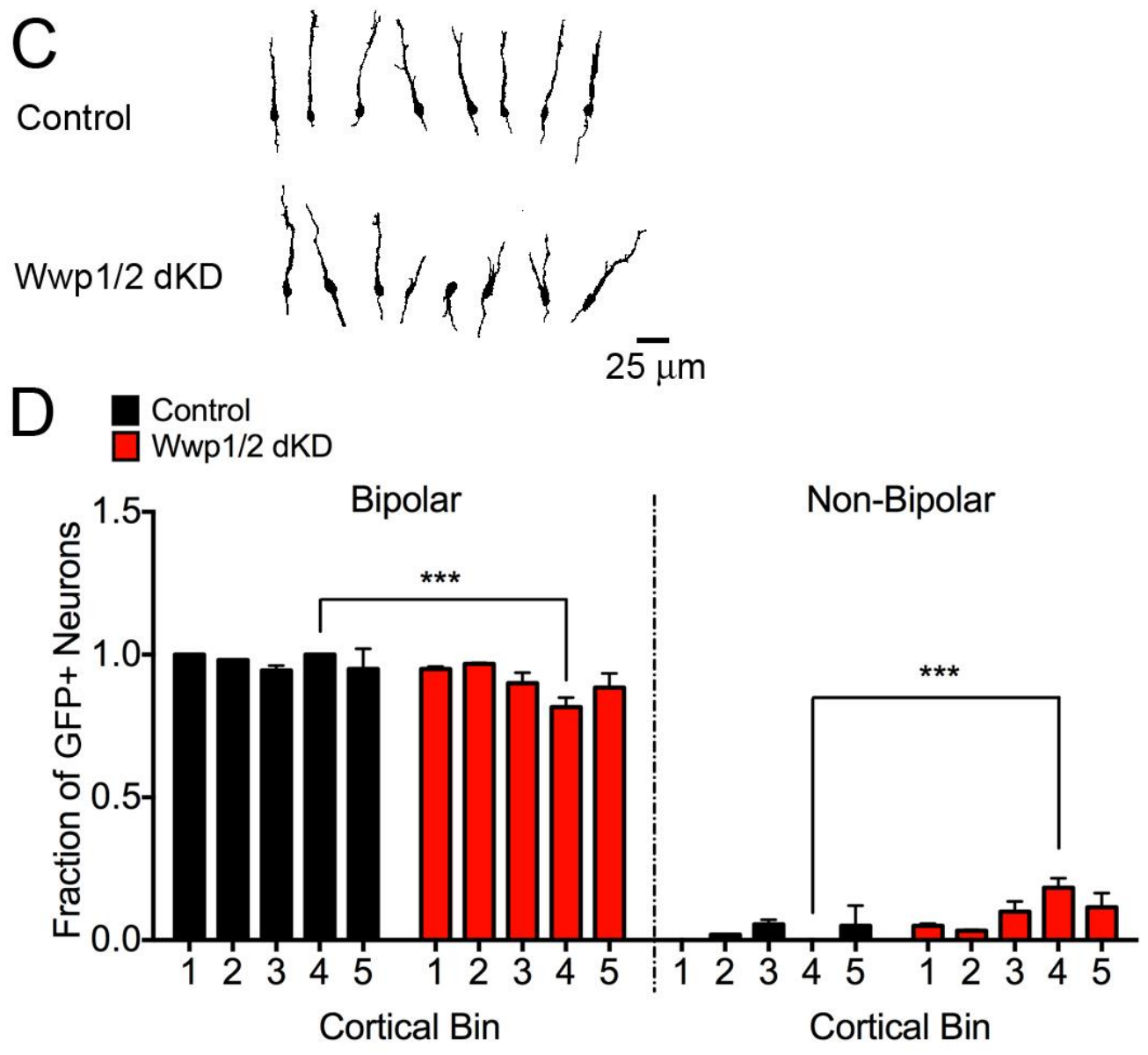

Figure 6. Knock-down of Wwp1 and Wwp2 follows with aberrant distribution and polarity defects in newborn neurons in vivo. (A) Representative images of DAPI signals (left panels), and immunostaining with anti-GFP antibody (right panels) in P0 cortices of wild type mice. Cortical progenitors were transfected in utero at E14.5 with plasmid encoding control non-silencing sh-RNA (upper panel), or cotransfected with two plasmids 
carrying sh-RNAs to downregulate Wwp1 and Wwp2 (lower panel). Images represent maximum projections of Z-stacks through $100 \mu \mathrm{m}$ thick coronal cryosections, stained with DAPI (left), or with anti-GFP antibody (right). Scale bar, $100 \mu \mathrm{m}$. (B) Distribution of cortical neurons upon double knock-down of Wwp1 and Wwp2 (Wwp1/2 dKD). Images of cortices were divided into 5 equal bins, and the relative number of neurons in each bin counted. Results are represented as averages \pm S.D. Control, $n=2$ brains; Wwp $1 / 2 \mathrm{dKD}, \mathrm{n}=2$ brains; $* * * p<0.001$, two-way ANOVA with Bonferroni post-hoc test. (C) Tracings of representative neurons in bin 3, and 4 of $\mathrm{P} 0$ control, or Wwp $1 / 2 \mathrm{dKD}$ brains. Scale bar, 25 $\mu \mathrm{m}$. (D) Quantification of neuronal polarities. Neurons were classified into either bipolar, or non-bipolar cells. Results are represented as averages \pm S.D. Control, $\mathrm{n}=2$ brains; Wwp $1 / 2$ $\mathrm{dKD}, \mathrm{n}=2$ brains; $* * * \mathrm{p}<0.001$, two-way ANOVA with Bonferroni post-hoc test.

Additionally, whereas axons projected from control pyramidal neurons run along parallel trajectories towards the ventricle, Wwp $1 / 2 \mathrm{dKD}$ neurons show loss of such organization of fibers, and display extensive branching of neurites (Fig. 7A, enlarged square). Next, we examined the morphology of individual neurons in P10 brains. At this stage of development, cortical neurons of layer II/III are highly polarized, with the main apical dendrite projecting towards the pial surface, and the axon emerging from the opposite pole of the soma [253] (Fig. 7C, top panel). Radially arrayed control neurons with such a pyramidal shape comprised $99.3 \pm 1.3 \%$ of all neurons in bin $1,93 \pm 5.6 \%$ in bin $2,100.0 \%$ in bin 3 , and 4 , and $46.0 \pm 12.5 \%$ in bin 5 (Fig. 7D). Upon Wwp1/2 dKD, we observed a significant decrease in the frequency of pyramidal neurons at the expense of cells with no prominent apical dendrites or axon (Fig. 7C, bottom panel, cells $1-4$, and 6 from the left, Fig. 7D). Of note, several pyramidal neurons displayed misoriented apical dendrite in relation to the pial surface (Fig. 7C, bottom panel, cell 7 from the left). Consequently, Wwp1/2 dKD neurons with pyramidal characteristics comprised $89.6 \pm 2.9 \%$ of all neurons in bin $1,66.7 \pm 4.6 \%$ in bin $2,74.1 \pm 2.9 \%$ in bin $3,75.3 \pm 11.2 \%$ in bin 4 , and $24.2 \pm 18.5 \%$ in bin 5 (Fig. 7D). Interestingly, polarity aberrances were the most prominent in Wwp $1 / 2 \mathrm{dKD}$ neurons found in deeper cortical layers, probably due to migration delay, as compared to neurons that distributed in layer II/III. Altogether, knock-down of Wwp1, and Wwp2 abrogates axon specification in primary hippocampal neurons paralleled by persistent distribution defects and axon-dendrite polarity disruption in cortical neurons in vivo. 


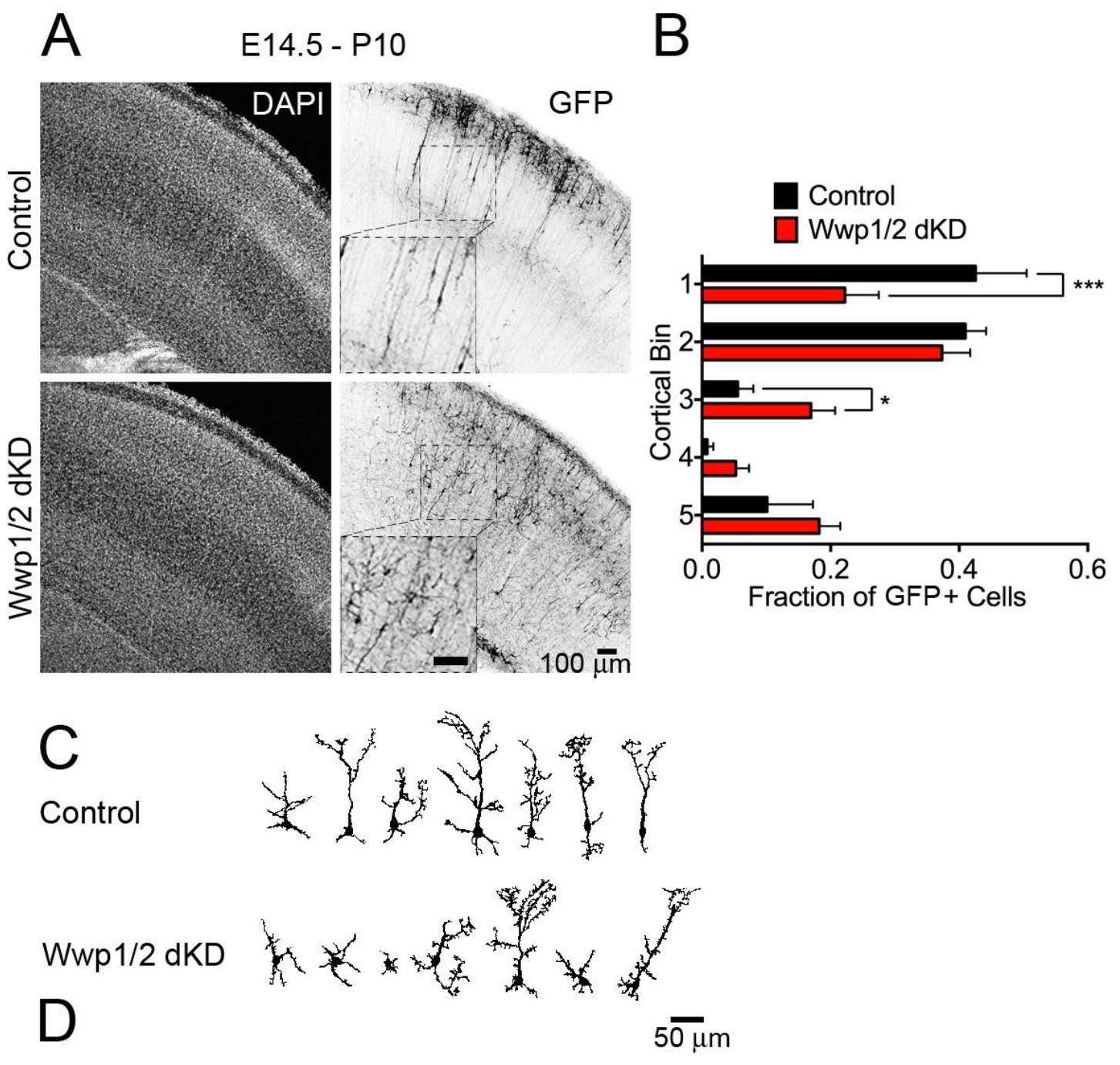

Control

Wwp1/2 dKD

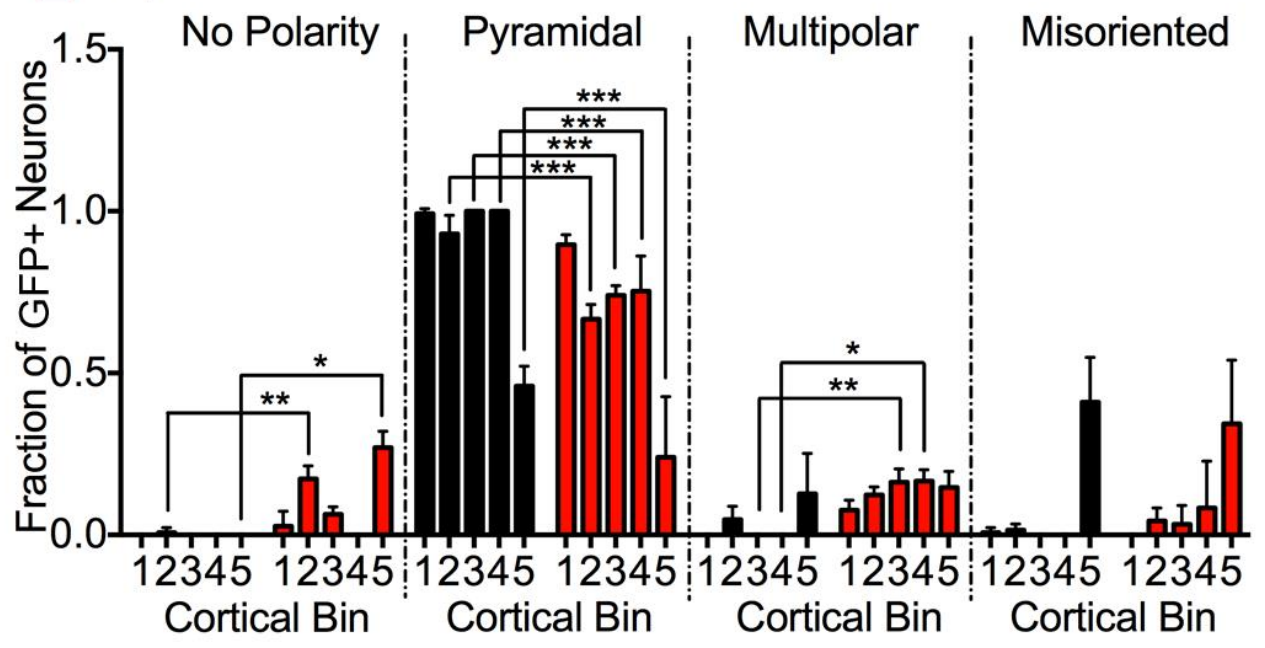

Figure 7. Knock-down of Wwp1 and Wwp2 leads to persistent distribution aberrances and disrupts neuronal polarity in vivo. (A) Representative images of DAPI staining (left panels), and GFP immunostaining (right panels) in P10 cortices of wild type mice. Neurons were electroporated in utero at E14.5 with a plasmid encoding for myr-Venus and control non-silencing sh-RNA (upper panel), or with two plasmids, for expression of myr-Venus 
and sh-RNAs targeting Wwp1 and Wwp2 (lower panel). Images represent maximum projections of stacks through $100 \mu \mathrm{m}$ thick coronal cryosections, immunostained with an antibody against GFP. Scale bar, $100 \mu \mathrm{m}$. (B) Distribution of neurons in the cortex upon Wwp1/2 dKD in vivo. The number of counted neurons in each bin was expressed as a fraction of total number of electroporated nerve cells. Results are represented as averages \pm S.D. Control, $\mathrm{n}=4$ brains; Wwp1/2 dKD, $\mathrm{n}=3$ brains; *** $\mathrm{p}<0.001,{ }^{*} \mathrm{p}<0.05$, two-way ANOVA with Bonferroni post-hoc test. (C) Tracings of representative neurons in bin 3 and 4 in P10 brains electroporated with control sh-RNA or sh-RNA for KD of Wwp1 and Wwp2. Scale bar, $50 \mu \mathrm{m}$. (D) Classification of neurons based on polarity upon Wwp1/2 dKD in vivo. Neurons were categorized as 'No Polarity', (neurons with no prominent dendrite or axon), 'Pyramidal' (neurons with a single prominent dendritic shaft projecting towards the pia), 'Multipolar' (neurons with more than one prominent dendrite or more than one axon), or 'Misoriented' (neurons with misoriented prominent apical dendrite), and the relative number of neurons of a given class was expressed as averages \pm S.D. Control, $n=4$ brains; Wwp1/2 dKD, $\mathrm{n}=3$ brains; $* * * \mathrm{p}<0.001, * * \mathrm{p}<0.01, * \mathrm{p}<0.05$, two-way ANOVA with Bonferroni post-hoc test.

\subsubsection{Genetic deletion of Wwp1 and Wwp2 leads to disturbances in axon acquisition in primary hippocampal neurons and disrupts polarity of cortical pyramidal neurons in vivo}

In order to corroborate if pronounced polarity aberrances in neurons upon Wwp $1 / 2 \mathrm{dKD}$ is a specific consequence of the loss of both ligases, we took advantage of $W w p 1^{\mathrm{f} / \mathrm{f}} ; W w p 2^{\mathrm{f} / \mathrm{f}}$ transgenic mice. In this mouse line, critical exons of $W w p 1$ and $W w p 2$ genes are flanked by loxP sequences. Consistent with deficiencies characterized for Wwp1/2 dKD, $39 \%$ of $W w p 1^{\mathrm{f} / \mathrm{f}} ; W w p 2^{\mathrm{f} / \mathrm{f}}$ primary hippocampal neurons expressing Cre recombinase displayed multiple axons versus only $15 \%$ of control $W w p 1^{\mathrm{f} / \mathrm{f}} ; W w p 2^{\mathrm{f} / \mathrm{f}}$ nerve cells expressing GFP endowed with multiple axons (Fig. 8A, and 8B). To support that the axon acquisition disturbances are indeed induced by loss of both Wwp ligases from the brain cells, we performed biochemical analyses in control $W w p 1^{+/+} ; W w p 2^{+/+}$and conventional double knockout $W w p 1^{-/-} ; W w p 2^{-/-}$mice. For this purpose, we crossed $W w p 1^{\mathrm{f} / \mathrm{f}} ; W w p 2^{\mathrm{f} / \mathrm{f}}$ with the E2A-Cre driver line in which Cre recombinase is expressed ubiquitously, therefore generating conventional $W w p 1^{-/-} ; W w p 2^{-/-}$. Western blotting using antibodies against Wwp1 and Wwp2 (Fig. 8C) confirmed complete depletion of both ligases from Wwp1 $1^{-/-}$; Wwp2 $2^{-/-}$ mouse brains, indicating that this strategy lead to efficient knockout of $W w p 1$ and $W w p 2$. 


\section{A}

a. $W w p 1^{1 / f} ; W w p 2^{f / f}+E G F P$
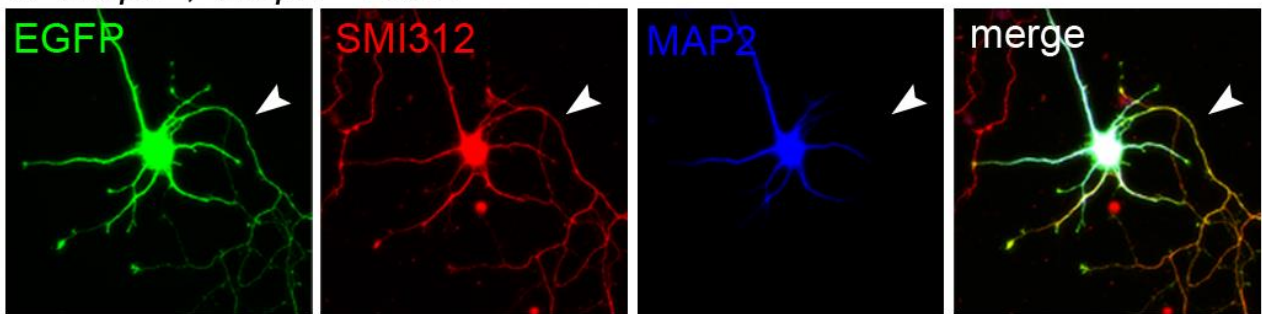

b. $W w p 1^{1 / f} ; W w p 2^{f / f}+E G F P-i C r e$
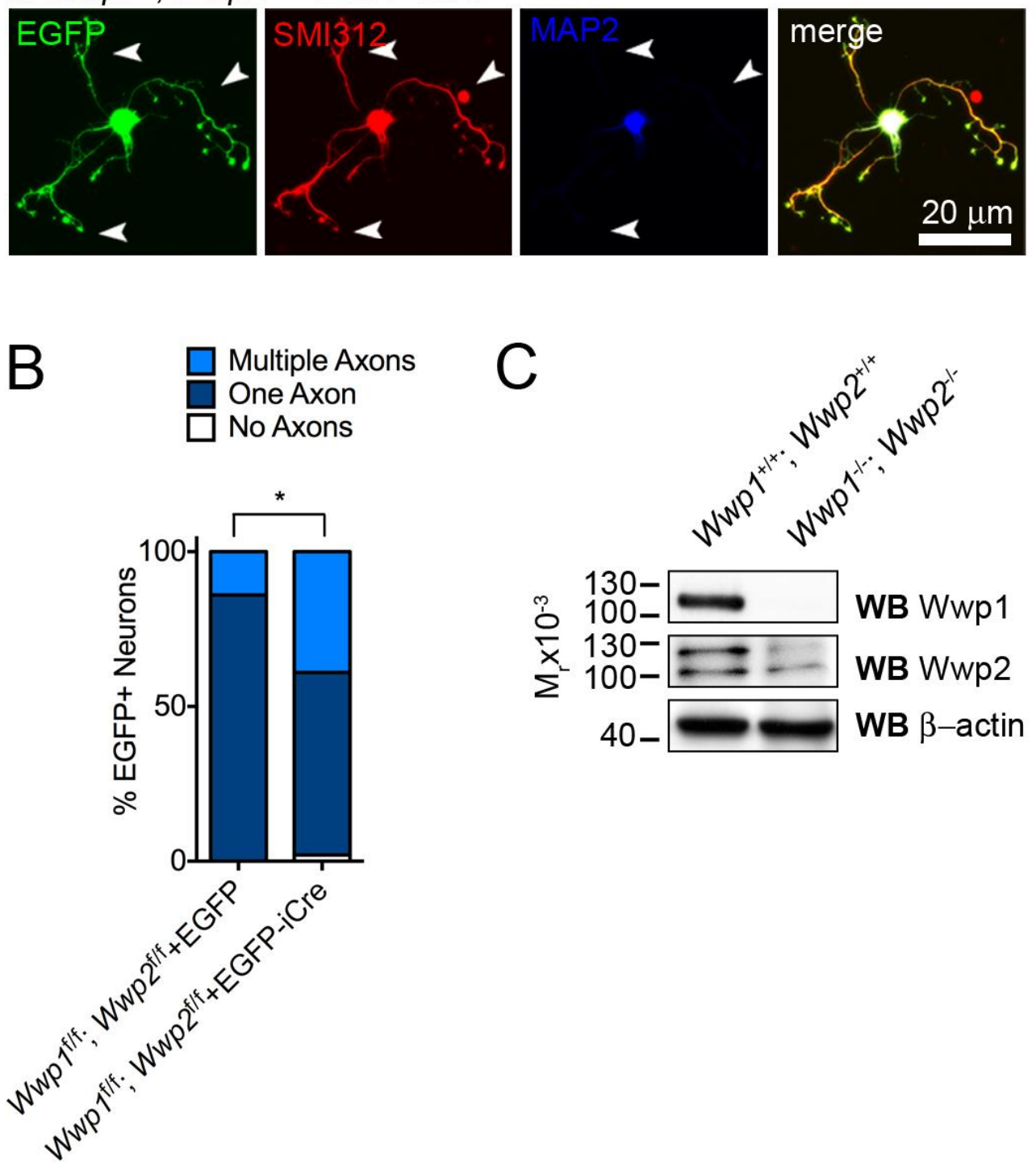

Figure 8. Disturbances in axon acquisition upon double knockout of $W w p 1$ and $W w p 2$ in primary hippocampal neurons. (A) Images of representative primary hippocampal neurons prepared from $W w p 1^{\mathrm{f} / \mathrm{f}} ; W w p 2^{\mathrm{f} / \mathrm{f}}$ mice. Neurons were transfected with an expression plasmid encoding EGFP as a control (a), or Cre recombinase to knockout $W w p 1$ and $W w p 2$ (b), fixed at DIV7, and immunostained for axonal and dendritic marker proteins. Arrowheads indicate axons. Scale bar, $20 \mu \mathrm{m}$. (B) Quantification of the number of axons projected from a single neuron. Control, $W w p 1^{\mathrm{f} / \mathrm{f}} ; W w p 2^{\mathrm{f} / \mathrm{f}}+\mathrm{EGFP}, \mathrm{n}=21$; double $W w p 1$ 
and $W w p 2$ knockout, $W w p 1^{\mathrm{f} / \mathrm{f}} ; W w p 2^{\mathrm{f} / \mathrm{f}}+$ EGFPiCre, $\mathrm{n}=41$ neurons; $* \mathrm{p}<0.05$, Chi-square test. (C) Validation of the loss of Wwp1 and Wwp2 by Western Blotting in cortical lysates prepared from $W w p 1^{+/+} ; W w p 2^{+/+}$, and $W w p 1^{-/-} ; W w p 2^{-/-} \mathrm{P} 7$ mice.

Knock-down of Wwp ligases in cortical neurons leads to severe morphological defects in vivo. Hence, we next studied the impact of genetic depletion of $W w p 1$ and $W w p 2$ in neuronal progenitors by transfecting plasmids encoding GFP (control), or GFP and Cre recombinase ( $W w p 1 / 2 \mathrm{dKO}$ ) by in utero electroporation in $W w p 1^{\mathrm{f} / \mathrm{f}} ; W w p 2^{\mathrm{f} / \mathrm{f}}$ mice (Fig. 9A). The $W w p 1 / 2$ dKO neurons exhibited slight accumulation of cells in deeper cortical layers (bins 3 and 4, Fig. 9B). The impact of $W w p 1$ and $W w p 2$ loss on neuronal morphology in deeper cortical layers was marked, and neurons with defective polarity resembled the ones described for WWP1/2 dKD neurons (Fig. 7C, lower panel, cells $1-4$, and 6 from the left). Whereas 91.7 $\pm 11.8 \%$ of control, GFP expressing neurons in bin 3 , and $100 \%$ in bin 4 presented a single apical dendrite, $30.2 \pm 22.5 \%$ of Wwp1/2 dKO neurons in bin 3 and $34.3 \pm 8.1 \%$ in bin 4 were categorized as pyramidal (Fig. 9D). Some neurons devoid of Wwp1 and Wwp2 manifested multiple dendritic shafts emerging from the soma (Fig. 9C, lower panel, cells 1 -5 , and cell 7 from the left, and Fig. 9D). Taken together, both knock-down of Wwp1 and Wwp2, and genetic depletion of $W w p 1$ and $W w p 2$ from neurons results in striking disturbances in specification of axon-dendrite polarity, and possibly leads to disruption of neuronal migration. In conclusion, both ligases are indispensable for acquiring proper morphology of nerve cells in the developing mouse brain. 


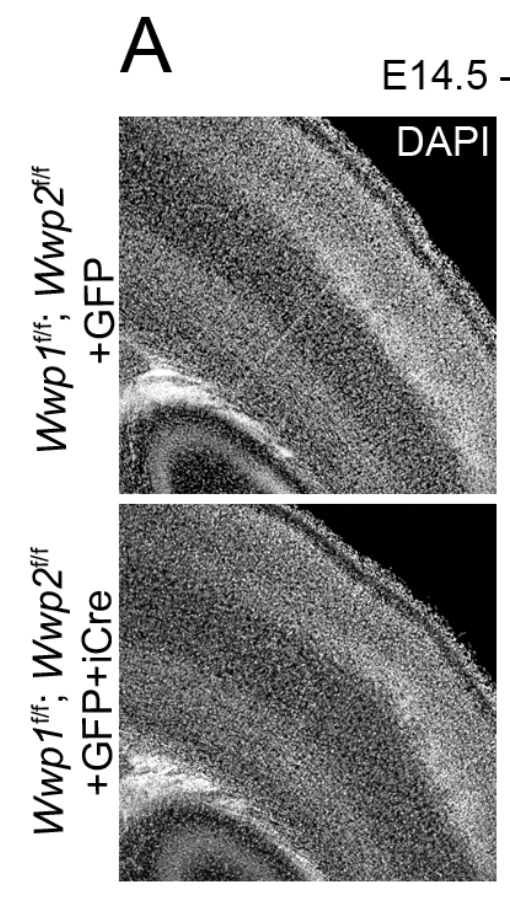

\section{B}

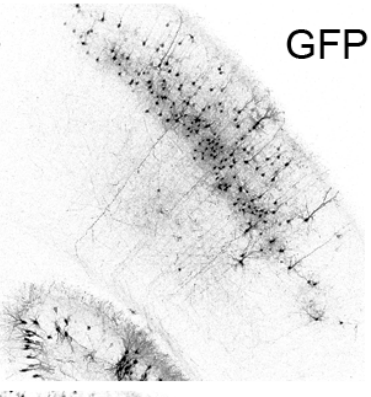

GFP

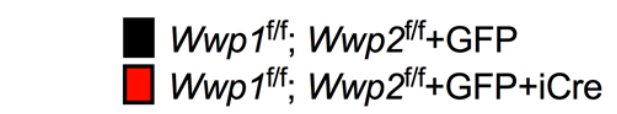

C

$W w p 1^{\text {fff; }} ; W w p 2^{\text {fff }}$
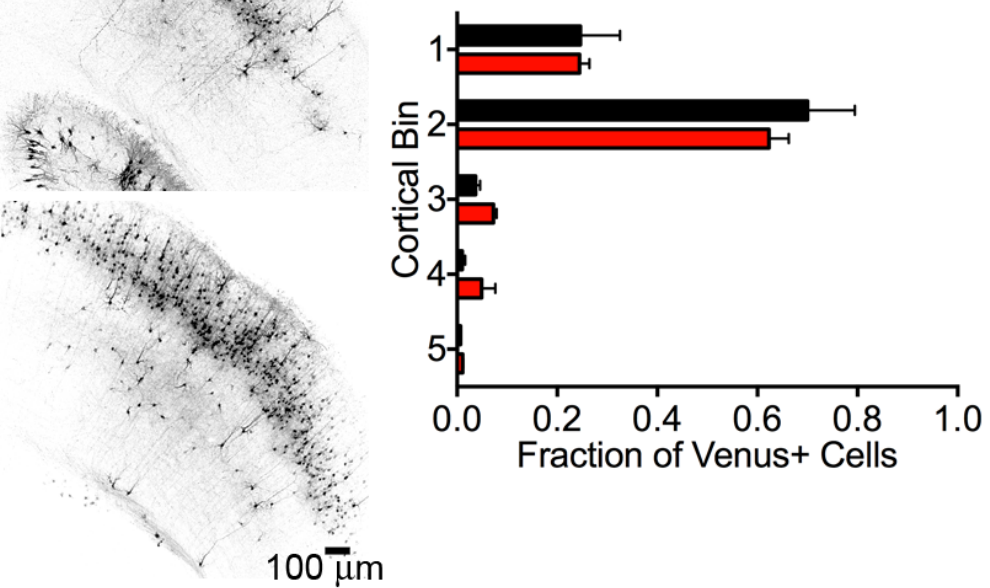

$\mu \mathrm{m}$

$$
\begin{aligned}
& \begin{array}{l}
\text { Wwp 1ff; } W w p 2 \\
+ \text { GFP+iCre }
\end{array}
\end{aligned}
$$

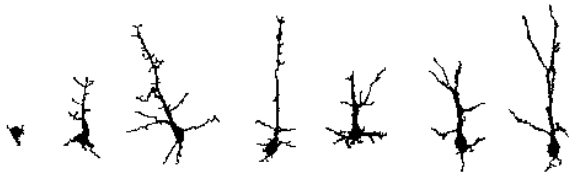

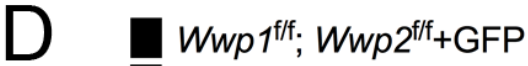

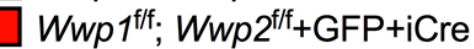

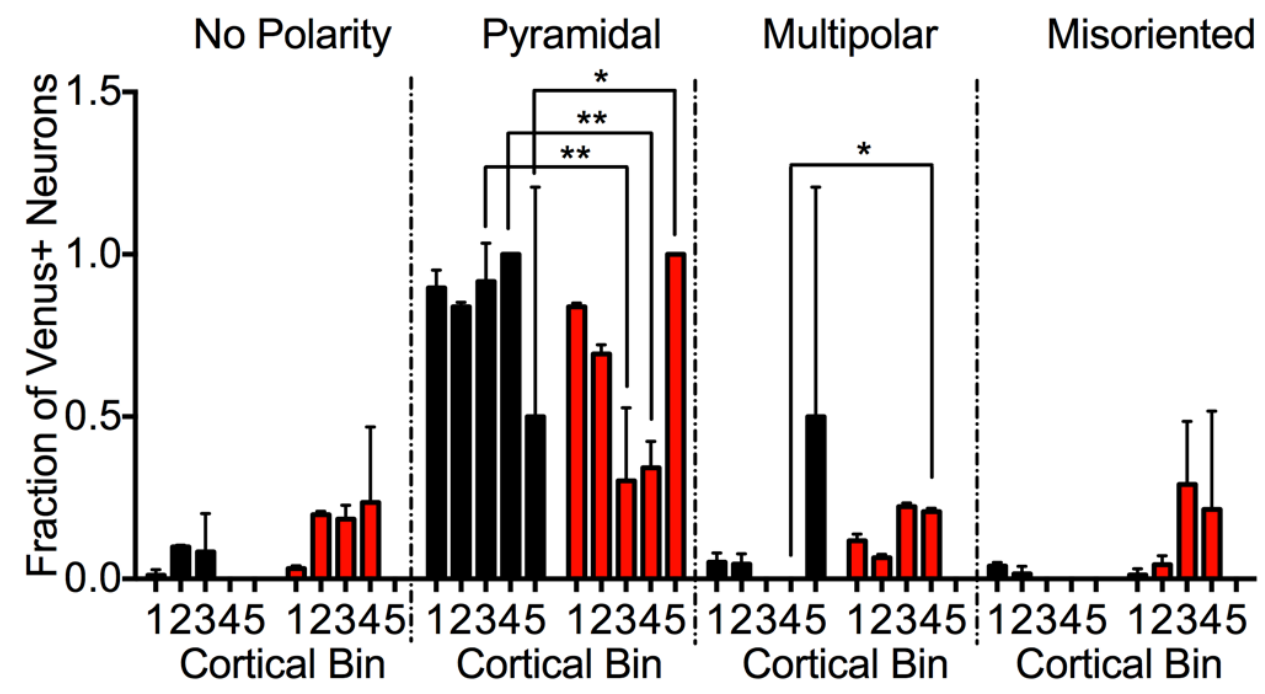

Figure 9. Knockout of $W w p 1$ and $W w p 2$ follows with severe neuronal polarity defects in vivo. (A) Representative images of DAPI (left panels), and GFP fluorescence signals (right panels) in P10 cortices of $W w p 1^{\mathrm{f} / \mathrm{f}} ; W w p 2^{\mathrm{f} / \mathrm{f}}$ mice. Neurons were electroporated in utero at E14.5 with plasmids encoding myr-Venus and GFP (upper panel), or myr-Venus, GFP, and Cre recombinase (iCre) (lower panel) to induce double knockout of $W w p 1$ and $W w p 2$ 
(Wwp1/2 dKO). Scale bar, $100 \mu \mathrm{m}$. (B) Quantification of neuronal distribution upon $W w p 1 / 2$ $\mathrm{dKO}$ in vivo. Results are represented as averages \pm S.D. Control, $W w p 1^{\mathrm{f} / \mathrm{f}} ; W w p 2^{\mathrm{f} / \mathrm{f}}+\mathrm{EGFP}$, $\mathrm{n}=2$ brains; $W w p 1 / 2 \mathrm{dKO}, W w p 1^{\mathrm{f} / \mathrm{f}} ; W w p 2^{\mathrm{f} / \mathrm{f}}+$ EGFPiCre, $\mathrm{n}=2$ brains; two-way ANOVA with Bonferroni post-hoc test. (C) Tracings of representative control and Wwpl/2 dKO neurons in bin 3 and 4 of P10 brains. Scale bar, $50 \mu \mathrm{m}$. (D) Classification of neuronal polarities upon $W w p 1 / 2 \mathrm{dKO}$. Results are represented as averages \pm S.D. Control, $W w p 1^{\mathrm{f} / \mathrm{f}}$; $W w p 2^{\mathrm{f} / \mathrm{f}}+$ EGFP, $\mathrm{n}=2$ brains; $W w p 1 / 2 \mathrm{dKO}, W w p 1^{\mathrm{f} / \mathrm{f}} ; W w p 2^{\mathrm{f} / \mathrm{f}}+$ EGFPiCre, $\mathrm{n}=2$ brains; $* * \mathrm{p}<0.01, * \mathrm{p}<0.05$, two-way ANOVA with Bonferroni post-hoc test.

\subsection{4. miR-140 is expressed in nerve cells}

Interestingly, $W w p 2$ gene harbors a locus of miR-140, and concomitant expression of truncated splicing isoform of Wwp2 and its intron-retained miR-140 has been reported in non-neuronal tissue [172]. microRNAs (miRNAs) comprise an evolutionarily conserved molecular mechanism to post-transcriptionally control gene expression [144,254,255]. Intronic miRNAs have been functionally linked with their host genes [183]. It has been proposed that upon concomitant transcription of host gene and its intragenic miRNAs, both genes are involved in one molecular pathway either supporting one another [256] or functioning as mutual antagonists [257]. Indeed, Wwp2 and miR-140 are both expressed in dividing and differentiating cells, such as chondrocytes [172], or stem cells [258], where they play crucial roles regulating their targets post-translationally and post-transcriptionally. To unravel if both Wwp2 and miR-140 undergo coordinated expression to regulate polarity formation in postmitotic neurons, we sought miR-140 in the central nervous system. We performed in situ hybridization on developing and adult brain sections with antisense probe directed against intronic $W w p 2$ sequence spanning miR-140 stem-loop, pri-miR-140 (Fig. $10 \mathrm{~A}$, top scheme). We detected strong hybridization signals in the ventricular zone of the developing cortical plate, ganglionic eminences (Fig. 10A), and throughout entire adult brain (Fig. 10B). However, rather than transcription of miR-140 precursor locus, it is the processing and maturation of pri-miR-140, that leads to generation of effector miRNA duplex $[259,260]$. We employed qRT-PCR using specific primers (Fig. 10C) to detect both mature miR-140 strands (-5p and $-3 p)$ in developing and adult mouse cortex (Fig. 10D). Interestingly, miR-140-3p displayed a distinct expression profile with early embryonic induction and rather stable postnatal level. In contrast, miR-140-5p was expressed at constant levels through the development and did not present such dynamic changes of expression. This indicates that miR-140-3p may serve as the guide miR-140 strand to silence mRNAs of genes critical during neuronal development at embryonic to perinatal stages. 

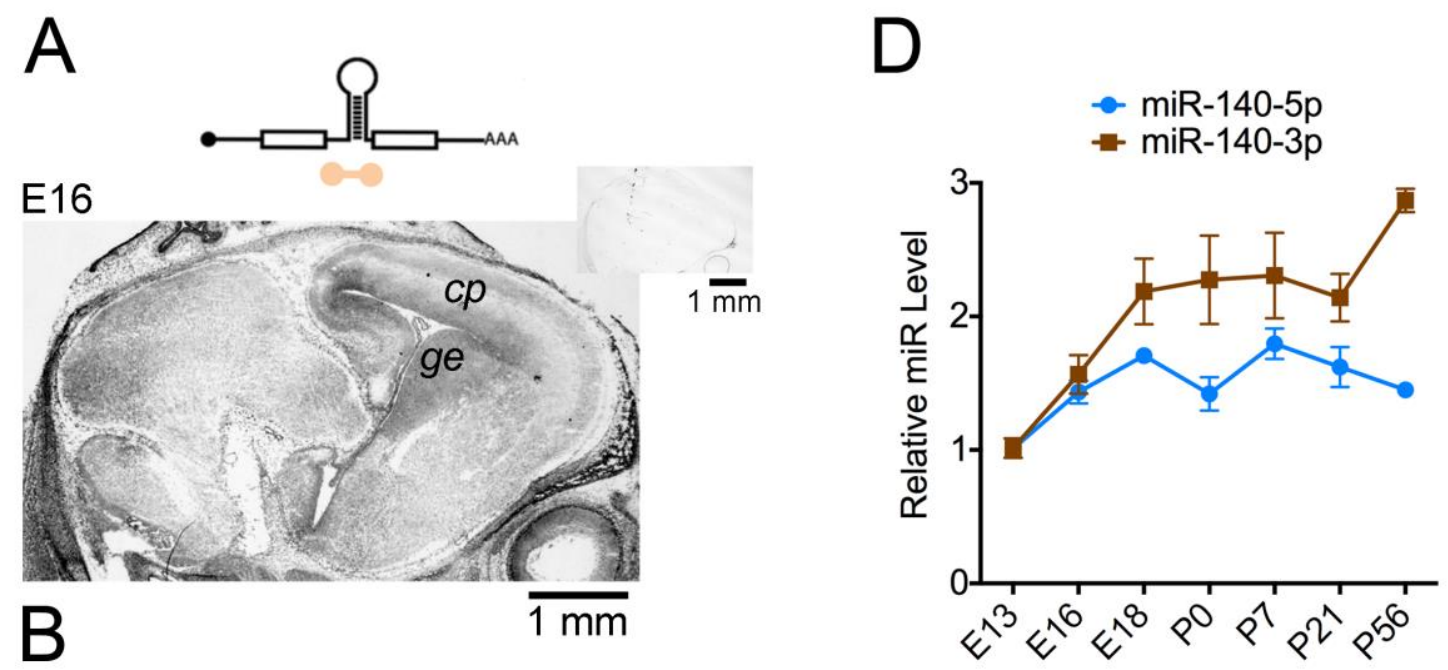

P20

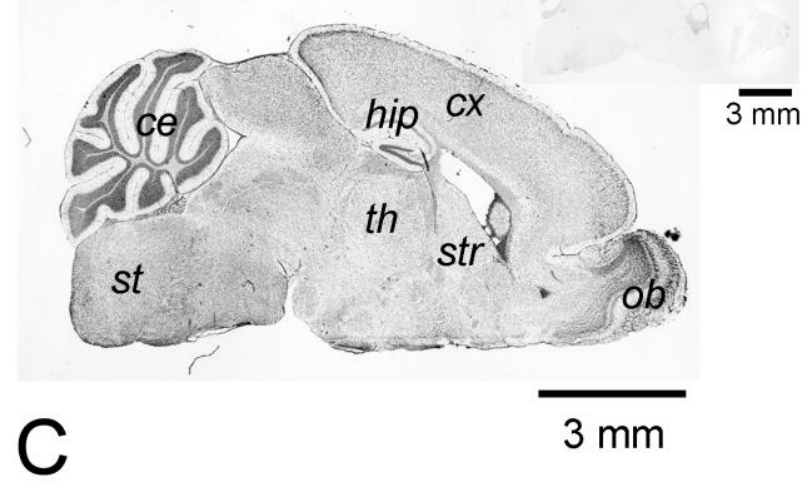

E

$\operatorname{miR}-140^{+/+}$ $\square \operatorname{miR}-140^{+1-}$ $\square \mathrm{miR}-140^{-1-}$
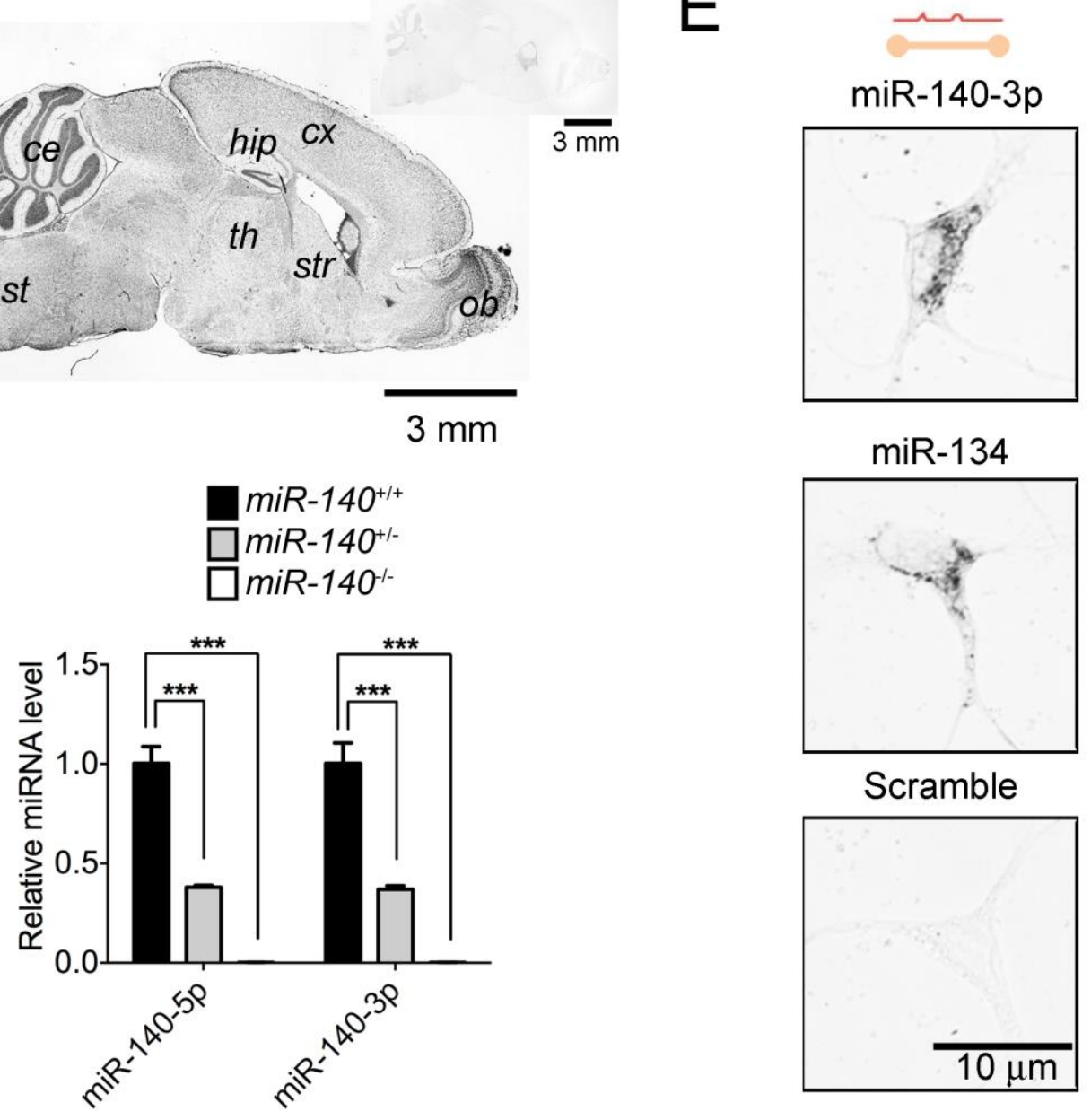

Figure 10. miR-140 is expressed in murine central nervous system. (A and B) Results of in situ hybridization of the antisense probe for intronic $W w p 2$ spanning miR-140 locus (upper scheme) on sagittal sections of E16 (A), and P20 (B) wild type mouse brain. Images in the top right inserts represent signals from the control sense probe; (A) cortical plate $(c p)$, ganglionic eminence ( $g e$ ); (B) cortex (cx), hippocampus (hip), cerebellum (ce), brain stem 
$(s t)$, thalamus $(t h)$, striatum (str), and olfactory bulb $(o b)$. (C) Validation of qRT-PCR primer specificity for miR-140-5p, and -3p. cDNA samples were prepared from total RNA extracted from cerebral cortices of $m i R-140^{+/+}, m i R-140^{+/-}$, and $m i R-140^{-/-}$mice at P21. The level of each miR-140 strand was expressed relative to component of spliceosome, U6 snRNA RNU6B. (D) Developmental profile of miR-140 expression in developing mouse cortex. Total RNA was isolated from cortices of mice of depicted age. The level of each miR strand was expressed relative to RNU6B, and to the miR level at E13. Results are represented as averages \pm S.E.M, $n=2$ independent experiments. (E) In situ hybridization with lockednucleic acid probes (upper scheme) for mature miR-140-3p (top panel), dendritic miR-134 (middle panel), and scramble control (bottom panel) in DIV7 primary hippocampal neurons. Scale bar, $10 \mu \mathrm{m}$.

To validate if developing neurons express miR-140-3p, and to study its cellular localization, we performed further in situ hybridization with locked nucleic acid probes on DIV7 primary mouse hippocampal neurons in collaboration with Dr. Tamara Rabe and Prof. Ahmed Mansouri (MPIbpc, Göttingen). Scramble probe was used as a negative control, and as a positive reporter, we used brain-enriched miR-134 [261]. As was the case for miR-134, at this developmental stage miR-140-3p did enrich neither in dendritic nor in axonal compartments, and localized at somatic endosomes (Fig. 10E). Taken together, miR-140 as well as $W w p 2$ is expressed in developing and adult murine neurons, implicating that miR140 may function in postmitotic neurons.

\subsubsection{Loss of $m i R-140$ phenocopies the knockout of $W w p 1$ and $W w p 2$ in regard to axon-dendrite specification in neurons and leads to aberrant distribution of developing nerve cells}

In order to test the hypothesis that miR-140 exerts synergistic or antagonistic effects on the role of its host $W w p 2$, we analyzed $m i R-140 \mathrm{KO}\left(\mathrm{miR}-140^{--}\right)$mice in terms of axon acquisition. Surprisingly, primary hippocampal neurons prepared from $m i R-140 \mathrm{KO}$ animals display strong defects in axon specification (Fig. 11A, a, and b) with $45 \%$ of $m i R-140 \mathrm{KO}$ neurons projecting multiple axons (Fig. 11B). Exogenous delivery of miR-140 to miR-140 $\mathrm{KO}$ neurons by transfecting a plasmid encoding for the precursor of the duplex (pre-miR140), reversed the aberrant axon acquisition phenotype (12 \% miR-140 KO neurons expressing exogenous miR-140 with multiple axons) (Fig. 11A, c, and d; 11B, c, and d). 


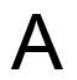

a. $\operatorname{miR}-140^{+/+}+$Scramble
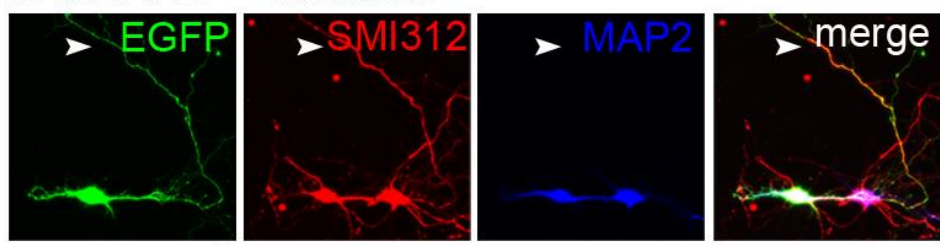

b. $\operatorname{miR}-140^{-1-}+$ Scramble
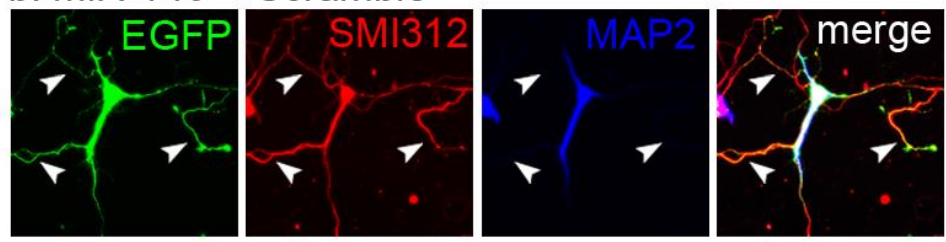

c. $\operatorname{miR}-140^{+/+}+\operatorname{miR}-140$
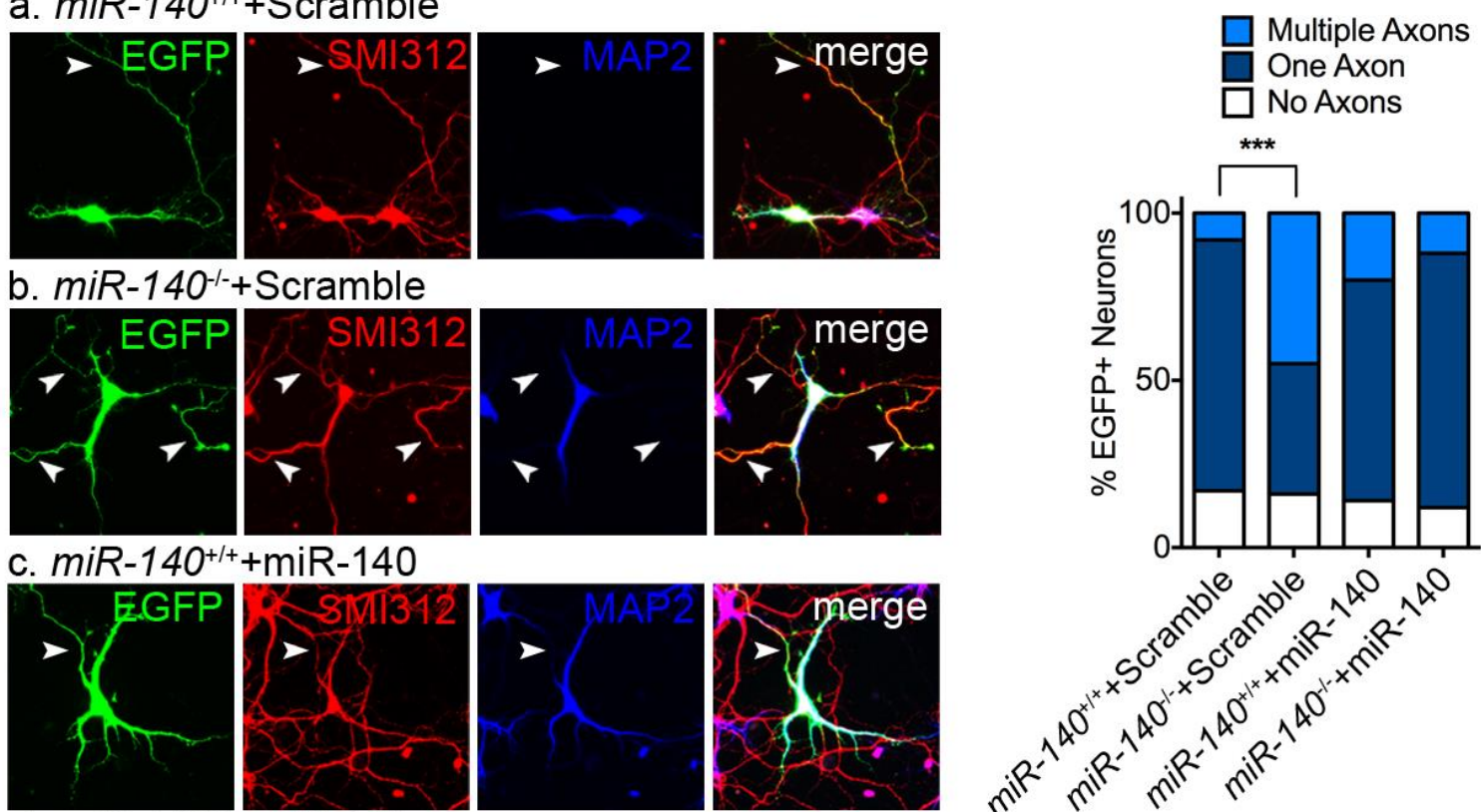

d. $\operatorname{miR}-140^{-1-}+\mathrm{miR}-140$
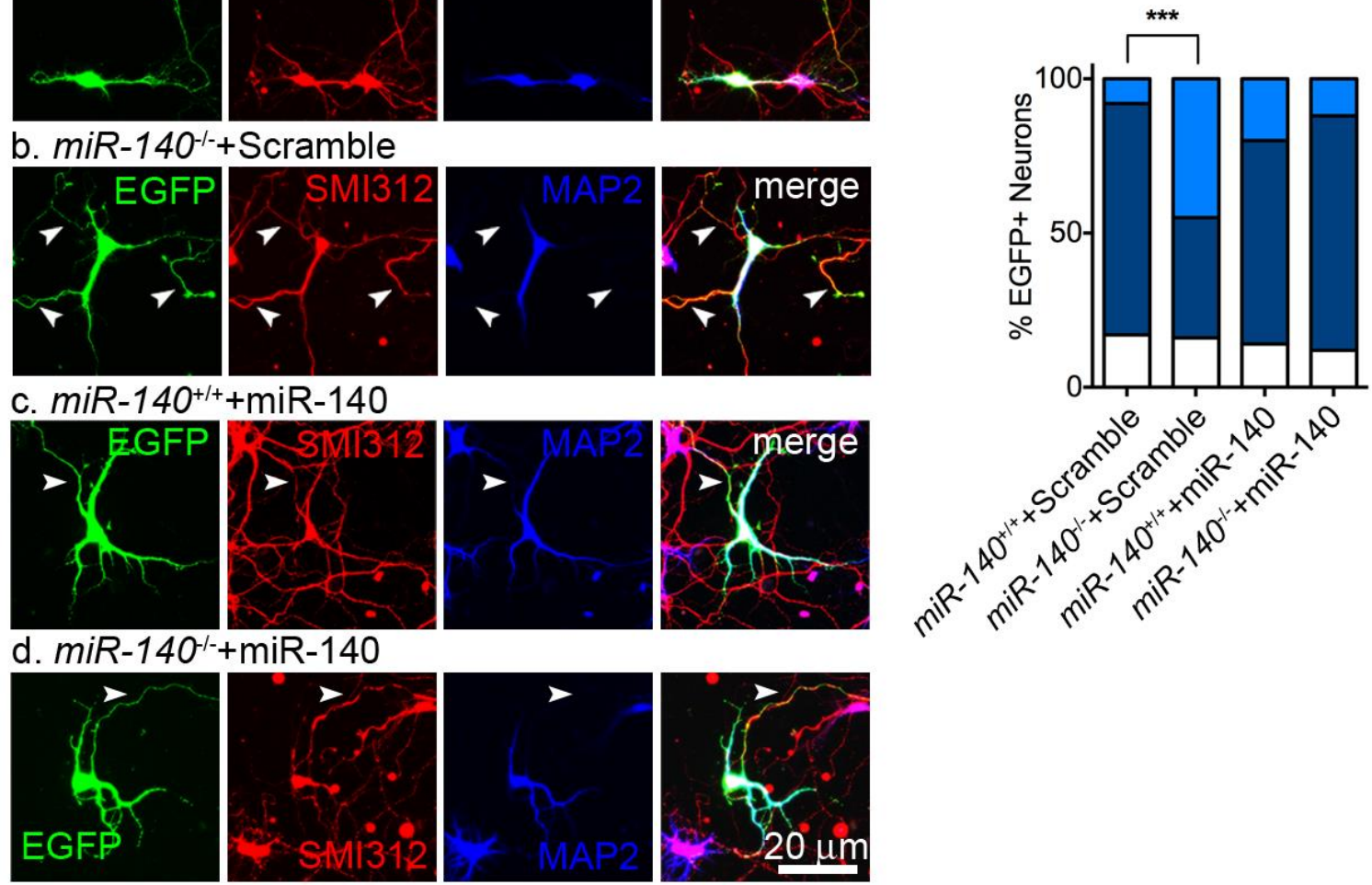

Figure 11. Loss of $m i R-140$ in neurons phenocopies loss of $W w p 1$ and $W w p 2$ regarding axon acquisition in primary hippocampal neurons. (A) Images of representative primary hippocampal neurons prepared from $m i R-140^{+/+}$(a, and c) or $m i R-140^{-/-}$(b, and d) mice. Neurons were transfected with expression plasmid encoding EGFP, and scramble miR (a, and b), or EGFP, and miR-140 (c, and d), and fixed at DIV7. Arrowheads point to SMI312positive axons. Scale bar, $20 \mu \mathrm{m}$. (B) Quantification of number of axons specified by individual neurons. $m i R-140^{+/+}, \mathrm{n}=41$ neurons; $m i R-140^{-/-}, \mathrm{n}=49$ neurons; $m i R-140^{+/+}+$ miR-140, $\mathrm{n}=35$ neurons; miR-140-- + miR-140, $\mathrm{n}=41$ neurons; $* * * \mathrm{p}<0.001$ by Chisquare test, and $* * * \mathrm{p}<0.001$, Fisher's exact test between the $m i R-140^{+/+}(\mathrm{a})$, and $m i R-140^{-}$ /- (b).

Next, we studied morphology and cortical distribution of P0 miR-140 KO neurons after in utero electroporation of plasmids encoding for GFP and Venus at E14.5 (Fig. 12A). The miR-140 KO neurons display altered distribution throughout cortical plate delay as compared to control nerve cells, suggesting a defect in neuronal migration. Majority of control neurons, $57.0 \pm 10.1 \%$, completed migration towards the pial surface, whereas only $20.0 \pm 7.0 \%$ of the miR-140 KO neurons was found in bin 1. Additionally, significantly more of $m i R-140 \mathrm{KO}$ neurons were located to bin $2(54.3 \pm 14.4 \%)$ as compared to $31.9 \pm$ 
$12.1 \%$ of control nerve cells (Fig. 12B). Moreover, miR-140 KO cells displayed strong defects in terms of morphology. Significantly more miR-140 KO neurons found in the deeper layers of cortical plate possessed multiple neurites directed towards the pia (Fig. 12C, lower panel, cells 1, and 2 from the left), or failed to specify a prominent neurite (Fig. 12C, lower panel, cell 3, and 9 from the left) as compared to the wild type cells. Consequently, majority of the control cells in the deeper layers of developing cortical plate presented bipolar morphology $(95.8 \pm 7.2 \%$ in bin 4 , and $83.3 \pm 23.6 \%$ in bin 5$)$, whereas only a small fraction of $m i R-140 \mathrm{KO}$ neurons were classified as bipolar $(39.5 \pm 21.2 \%$ in bin 4 , and $29.7 \pm 14.9$ $\%$ in bin 5) (Fig. 12D). Altogether, similarly to Wwp ubiquitin ligases, miR-140 governs axon-dendrite specification in primary hippocampal neurons, and is indispensable for proper polarity formation and proper distribution of cortical pyramidal nerve cells. 


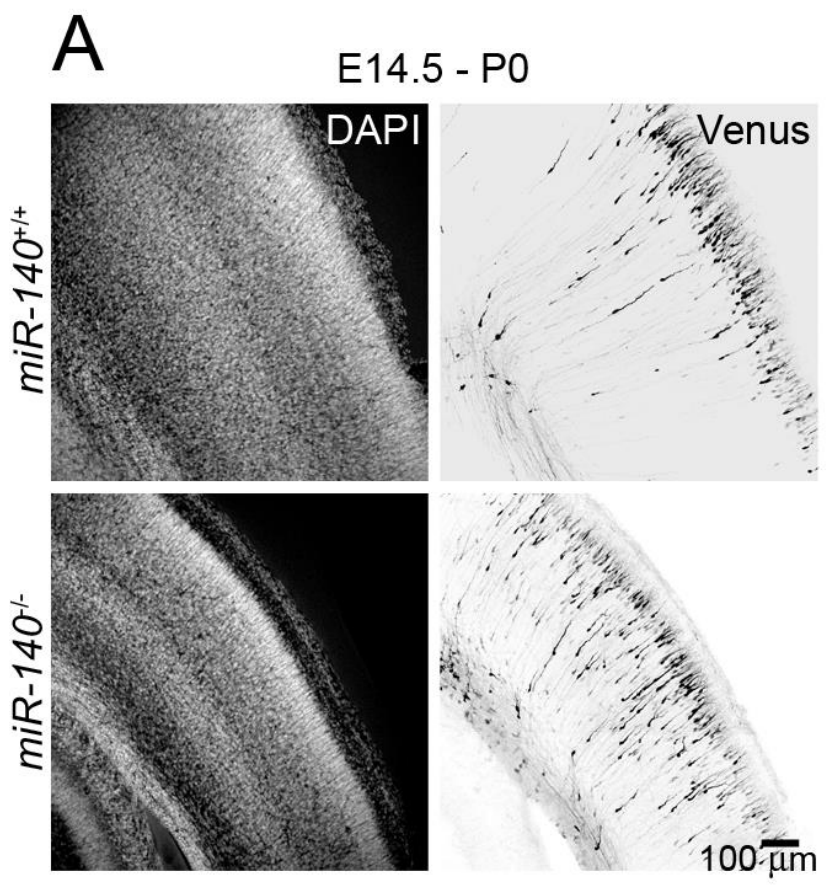

\section{B}
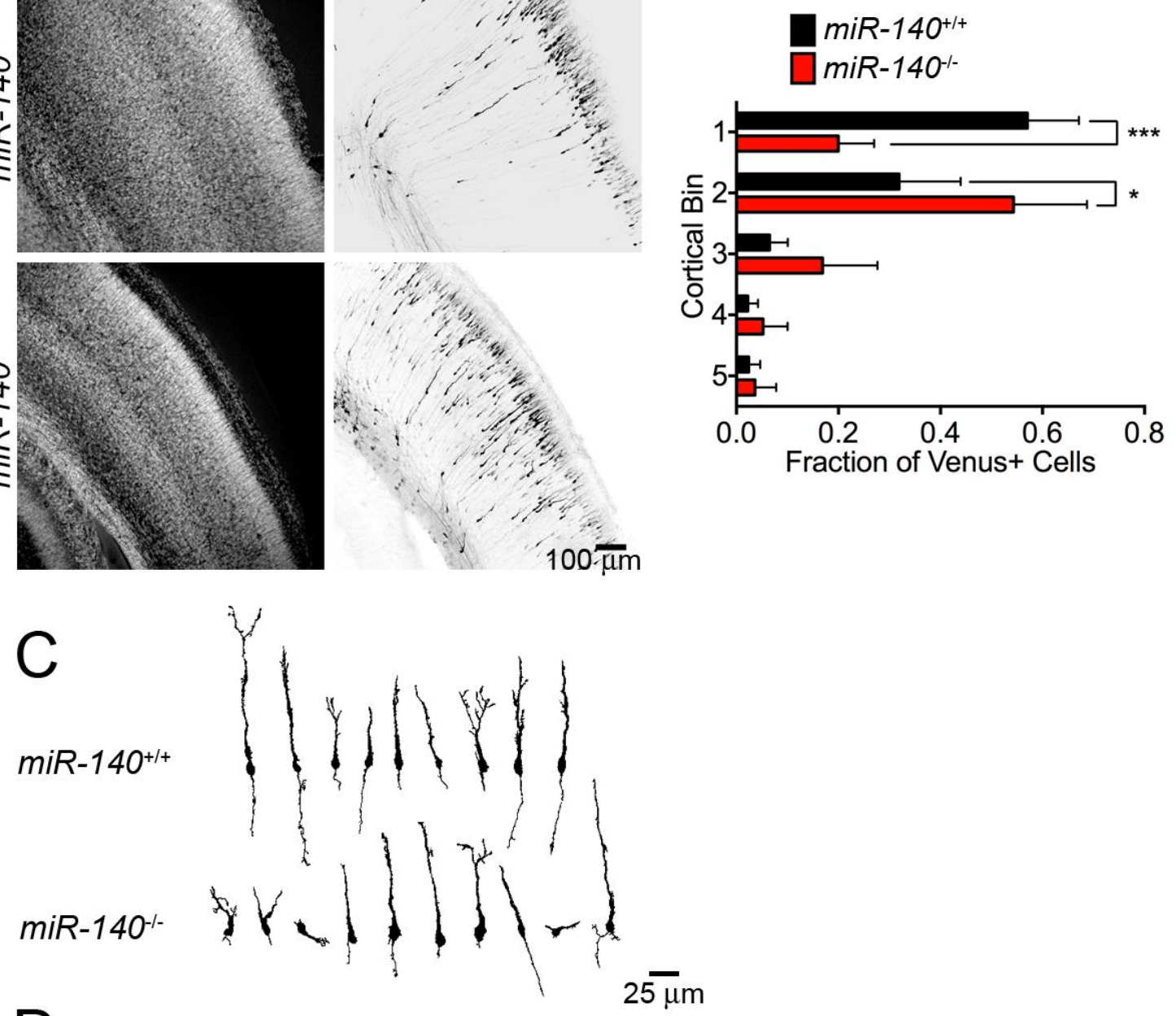

D

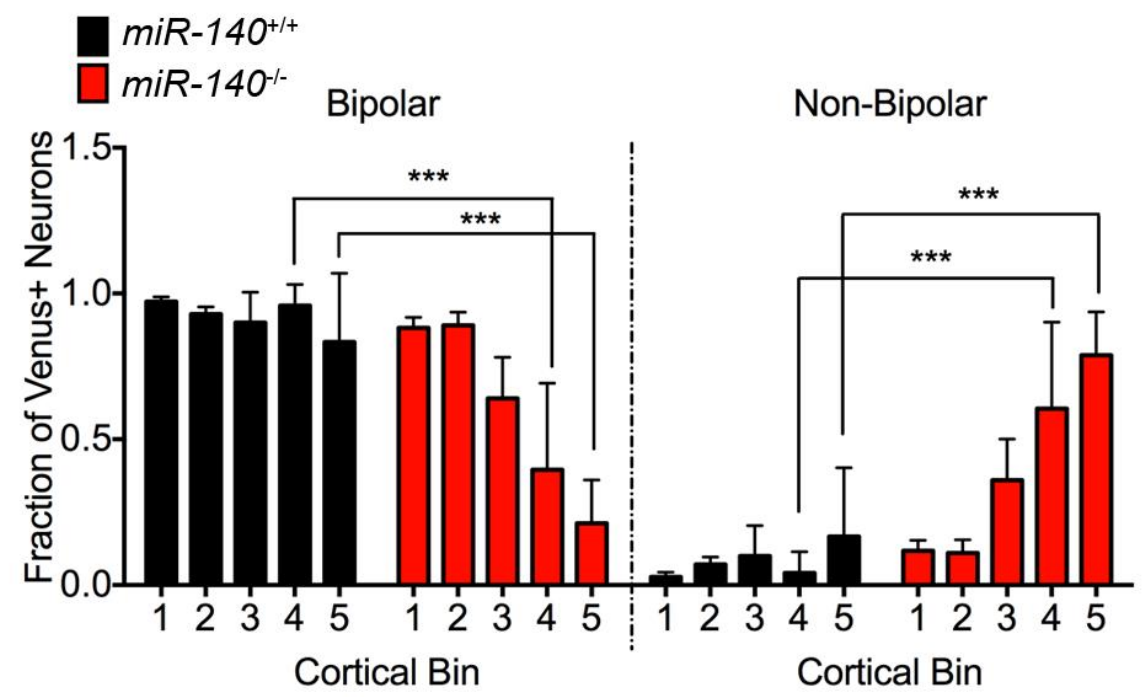

Figure 12. Knockout of miR-140 leads to abnormal neuronal distribution and disturbs polarity acquisition in newly born neurons in vivo. (A) Representative images of DAPI stain (left panels), and GFP/Venus fluorescence (right panels) in P0 cortices of $m i R-140^{+/+}$ 
(wild type, WT), or $m i R-140^{-/-}$(knockout, KO) mice. Neurons were transfected in utero at E14.5 with plasmids encoding myr-Venus and GFP. Scale bar, $100 \mu \mathrm{m}$. (B) Distribution of neurons in the cortical plate upon miR-140 KO. Bars represent averages of relative number of neurons \pm S.D. $m i R-140^{+/+}, \mathrm{n}=3$; miR-140 $0^{-/}, \mathrm{n}=4$ of analyzed brains; *** $\mathrm{p}<0.001$, * $\mathrm{p}<0.05$, two-way ANOVA with Bonferroni post-hoc test. (C) Tracings of representative neurons in bin 3 and 4 in $m i R-140^{+/+}$, and $m i R-140^{-/-}$brains at P0. Scale bar, $25 \mu \mathrm{m}$. (D). Classification of neuronal morphologies upon miR-140 KO. Bars represent averages of relative number of neurons \pm S.D. $m i R-140^{+/+}, \mathrm{n}=3$ brains; $m i R-140^{-/ /}, \mathrm{n}=4$ brains; ${ }^{* * *} \mathrm{p}$ $<0.001$, two-way ANOVA with Bonferroni post-hoc test.

\subsection{6. miR-140-3p, but not miR-140-5p is the mediator of axon specification in primary hippocampal neurons}

It has been reported, that in chondrocytes, miR-140-5p functions as the guide strand and regulates gene expression. In each miRNA, the strand with lower stability of base pairing from the second to fourth nucleotide is preferentially incorporated into miRNA-induced silencing complex to exert its gene silencing functions, whereas the remaining strand, the passenger strand, is degraded, or less biologically active [162,262,263]. RNA modifications, RNA-binding proteins, alternative Drosha processing mechanisms, or other molecular contexts can lead to an exchange of the guide strand of a given microRNA duplex in socalled 'arm switching' [200,264-266]. During cortical development, it is the expression of miR-140-3p strand that is induced early (Fig. 10D). In order to verify which strand of miR140 duplex is necessary for proper axon acquisition in neurons, together with Fritz Benseler (AGCT Lab, MPIem, Göttingen), we designed sponge constructs to downregulate individual miR-140 strands in neurons. RNA-sponge technology relies on complementary binding of targeted miRNA to its tandemly repeated binding sites. Upon delivery of the spongeexpressing plasmid to the neuron, targeted miRNA molecules are sequestered, and lead to de-inhibition of transcription of its target mRNAs. The specificity of sponge constructs towards each miR-140 strand was confirmed using PITA algorithm, allowing for computation of miRNA-target RNA thermodynamics [267]. 
A

a. Control

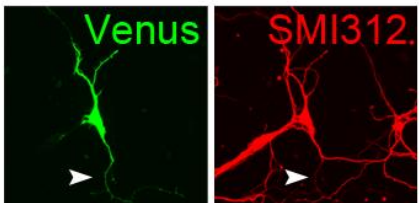

b. miR-140-5p-Sponge

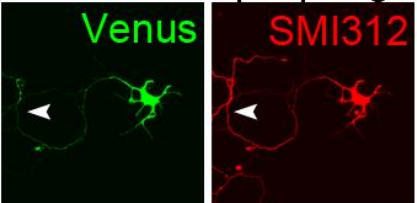

c. Control

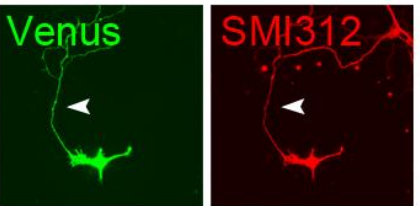

d. miR-140-3p-Sponge
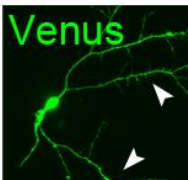

D

\section{a. Control}

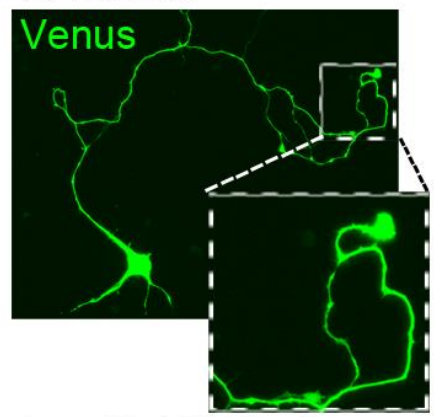

b. miR-140-3p-Sponge
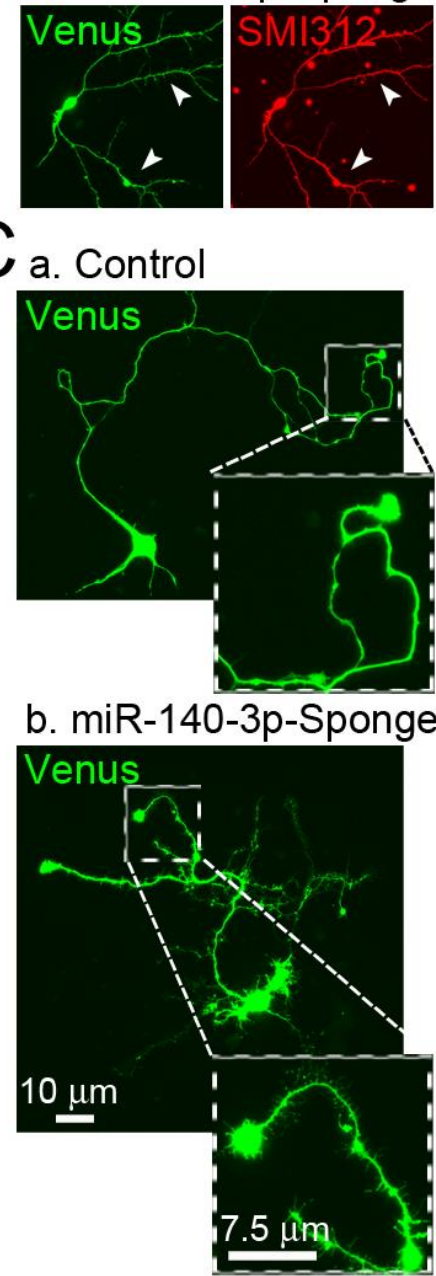

B
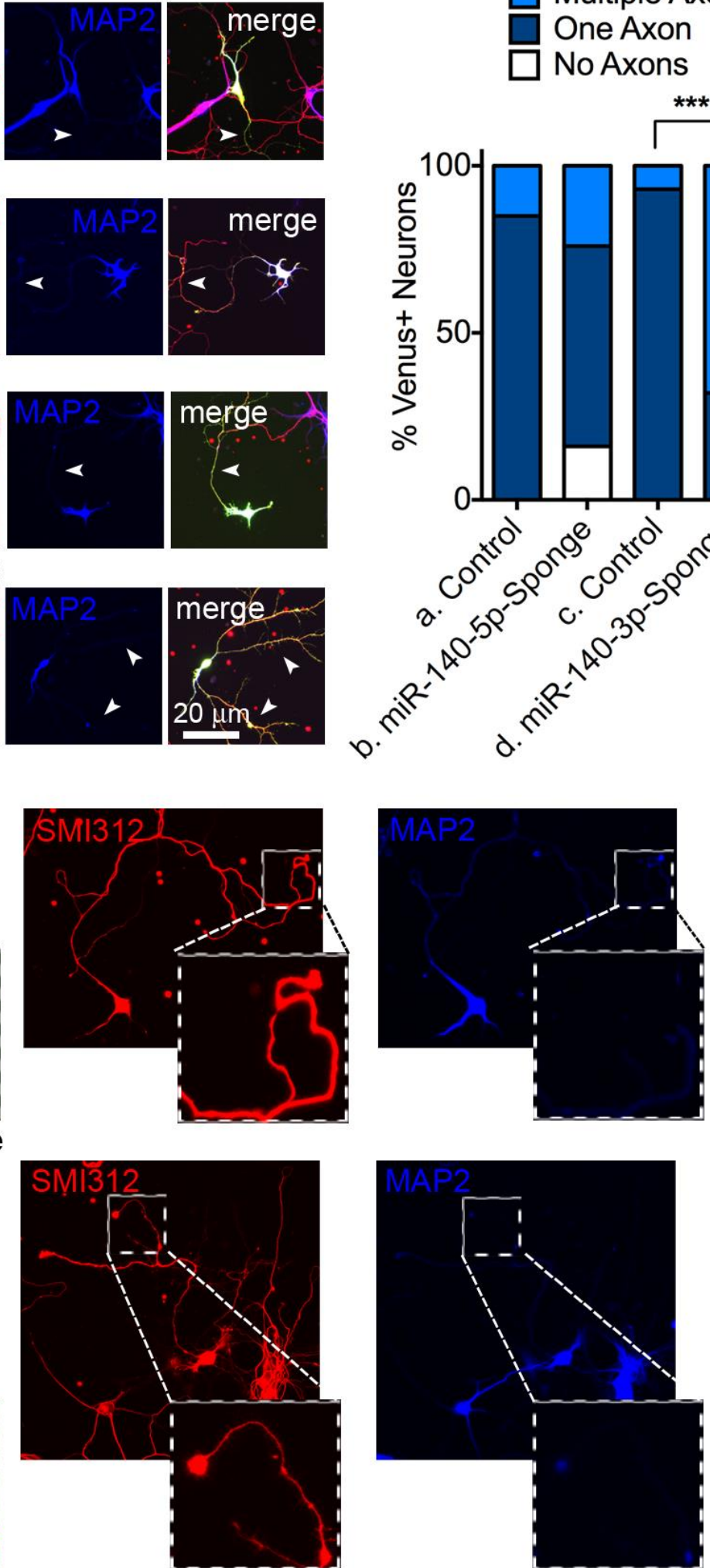

Figure 13. Knock-down of miR-140-3p, but not of miR-140-5p, leads to formation of multiple axons in primary hippocampal neurons. (A) Images of primary hippocampal neurons prepared from wild type mice. Nerve cells were transfected with control sponge (a), or miR-140-5p sponge (b), and with control sponge (c), or miR-140-3p sponge (d), and fixed at DIV7. All plasmids contained a Venus expression cassette that enabled tracing of 
individual neurites. Scale bar, $20 \mu \mathrm{m}$. (B) Quantification of the number of axons projected by sponge-transfected single neurons. Control, $\mathrm{n}=30$ neurons; miR-140-5p-Sponge, $\mathrm{n}=19$ neurons; control, $\mathrm{n}=20$ neurons; miR-140-3p-Sponge, $\mathrm{n}=25$ neurons; *** $\mathrm{p}<0.001$ by Chi-square test, and $* * * \mathrm{p}<0.01$, Fisher's exact test between neurons expressing control sponge (c), and neurons expressing miR-140-3p sponge (d). (C) Representative images of neurons expressing control (a), and miR-140-3p sponge (b) at DIV7.

While KD of miR-140-5p by expressing miR-140-5p-Sponge in wild type primary hippocampal cells did not induce any morphological and polarity defects (Fig. 13A, a, and b; B, a, and b), cells transfected with plasmid encoding miR-140-3p-Sponge exhibited a remarkable increase in neurons projecting multiple axons (Fig. 13A, c, and d; Fig. 13B; 7 \% of control cells, $68 \%$ miR-140-3p-Sponge expressing neurons). Those apparent axon acquisition aberrances were paralleled by numerous peculiar filopodia-like protrusions identified on some neurons expressing miR-140-3p-Sponge (Fig. 13C). Those findings demonstrate that $-3 p$ strand of miR-140 duplex is essential for normal axon specification operating in primary hippocampal neurons.

\subsubsection{Knock-down of miR-140-3p in cortical progenitors leads to persistent polarity aberrances and migration disturbances}

In order to study if aberrant axon acquisition in primary neurons upon acute knock-down of miR-140-3p is paralleled by morphological alterations of developing cortical neurons, we introduced plasmids encoding miR-140-3p-Sponge to cortical progenitors by in utero electroporation at E14, and sectioned mouse brains at P0 and P10 (Fig. 14A, and 15A). At P0, five days after transfection of the miR-140-3p knock-down construct to neurons, we observed no differences in neuronal distribution in cortical plate (Fig. 14B). Nonetheless, we distinguished remarkable aberrances in morphology of newborn neurons transfected with miR-140-3p-Sponge construct (Fig. 14C, lower panel, cells $1-3$ from the right with multipolar morphology; cells 4 and 5 from the right with misoriented leading processes). Notably, some of those neurons resembled abnormal newborn Wwp1/2 dKD nerve cells (compare Fig. 14C and Fig. 6C). Indeed, significantly less of miR-140-3p-Sponge expressing neurons retained bipolar morphology as compared to control Sponge expressing cells (Fig. 14D; miR-140-3p-Sponge, $80.4 \pm 8.6 \%$ bipolar neurons in bin 4 , and $75.7 \pm 17.1$ $\%$ in bin 5 of -3 p-Sponge expressing neurons; control Sponge, $95.2 \pm 5.5 \%$ in bin 4 , and $92.7 \pm 7.3 \%$ in bin 5$)$. 
In later stages of the development, at P10, miR-140-3p KD neurons consistently manifested morphological defects paralleled by strong aberrances in distribution of nerve cells in the cortex (Fig. 15A). Fig. 15B illustrates that significantly more of miR-140-3p-Sponge transfected neurons distributed in bin 1 (miR-140-3p-Sponge, $28.2 \pm 9.2 \%$; control Sponge, $19.9 \pm 5.3 \%$ of transfected neurons), and in bin 3 (miR-140-3p-Sponge, $10.3 \pm 4.7 \%$; control Sponge, $1.4 \pm 1.1 \%$ ) at the expense of nerve cells in bin 2 (miR-140-3p-Sponge, $58.2 \pm 7.3 \%$; control Sponge, $78.7 \pm 4.6 \%$ ). Morphological analysis of individual neurons revealed pronounced defects in polarity, mostly manifested by acquisition of multiple apical dendrites, or misorientation of the dendritic axis (Fig. 15C, lower panel, multiple apical dendrites: cell 1, 6-9, 13; misoriented dendritic axis towards the pia: cell 2, 3, 10, 14 from the left). Majority control neurons in bin 3 was pyramidal (66.7 $\pm 55.7 \%$ ), whereas only 18 $\pm 15.3 \%$ miR-140-3p-Sponge transfected neurons in bin 3 displayed normal pyramidal morphology (Fig. 15D).

Taken together, depletion of miR-140-3p from cortical nerve cells leads to pronounced detrimental effects on neuronal morphology and to disruption of neuronal distribution in the cortex. 

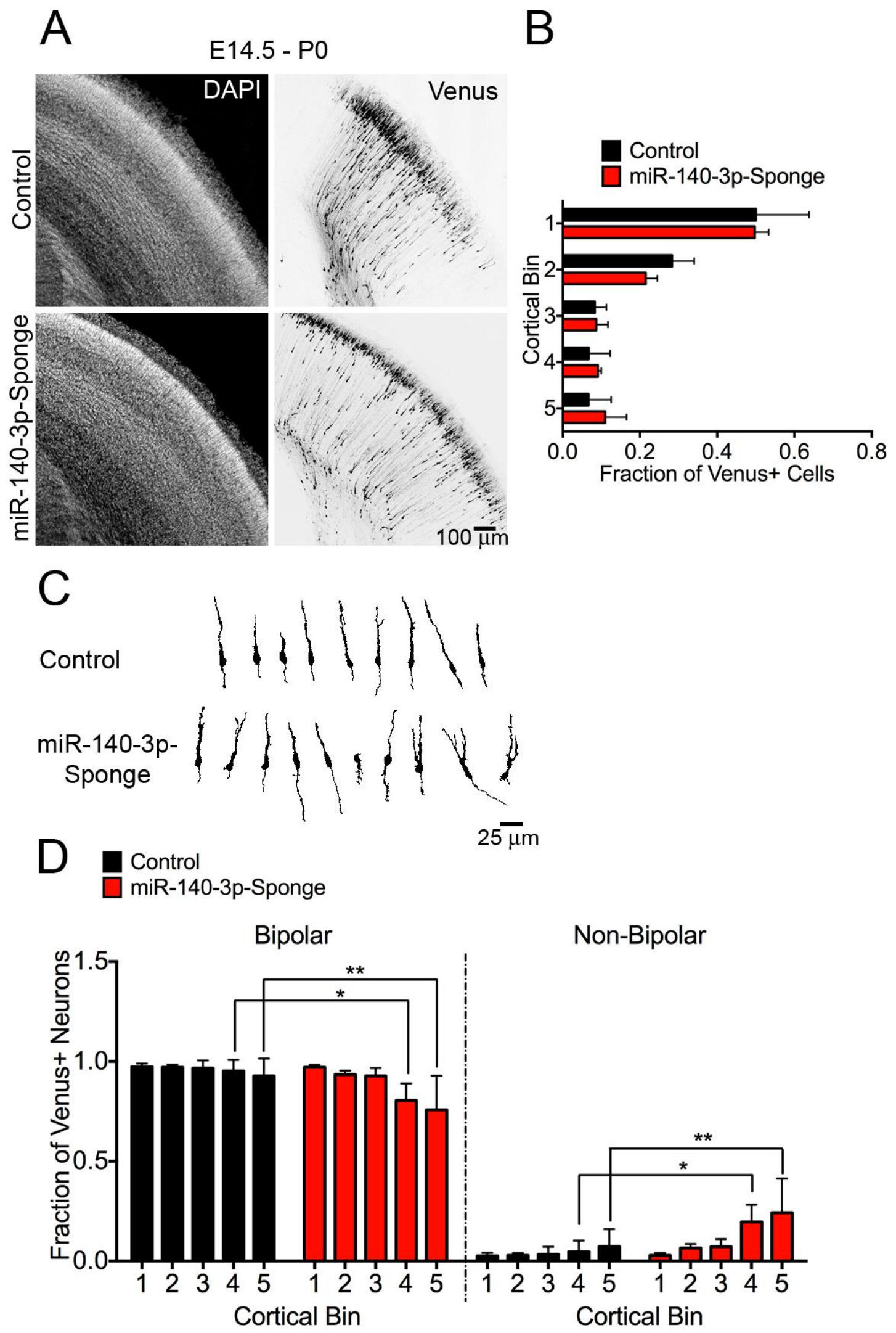

Figure 14. Knock-down of miR-140-3p in newborn neurons leads to polarity formation defects in vivo. (A) Representative images of DAPI staining (left panels), and Venus fluorescence (right panels) in P0 cortices of wild type mice. Neurons were electroporated in utero at E14.5 with plasmids encoding Venus, and control sponge (upper panel) or Venus, and miR-140-3p sponge (lower panel). Scale bar, $100 \mu \mathrm{m}$. (B) Neuronal distribution upon 
miR-140-3p KD. Results are represented as averages \pm S.D. Control, $n=7$ brains; miR-1403p-Sponge, $\mathrm{n}=7$ brains; two-way ANOVA with Bonferroni post-hoc test. (C) Tracings of representative control, and miR-140-3p KD neurons in bin 3, and 4 of P0 brains. Scale bar, $25 \mu \mathrm{m}$. (D) Classification of neuronal morphologies. Results are represented as averages \pm S.D. Control, $\mathrm{n}=7$ brains; miR-140-3p-Sponge, $\mathrm{n}=7$ brains; ${ }^{*} \mathrm{p}<0.05,{ }^{*} \mathrm{p}<0.01$ twoway ANOVA with Bonferroni post-hoc test.

\subsubsection{Altered distribution of neurons upon miR-140 depletion is not caused by aberrances in neurogenesis}

As the neurogenesis proceeds, progenitors in the ventricular and subventricular zone give birth to neocortical neurons of different cortical layers in a precisely controlled temporal fashion from E11.5 to E17.5 in mice [268]. Postmitotic neurons distribute in each layer of the developing neocortex depending on their identity specified at birth. Cut-like homeobox 1 (Cux1) is induced in immature neurons of layer II/III/IV and its expression prevails upon completion of cortical lamination [29]. Mutations in homeobox genes controlling laminar organization of the cortex lead to expansion or shrinkage of different cortical different layers due to aberrant identity specification of neurons.

Loss of polarity formation in neurons either driven by dKD of Wwp ligases or by miR-140$3 p$ is always paralleled by altered positioning of neurons in the cortex (Fig. 16A). In order to corroborate, that observed altered distribution of neurons is not due to alterations of neuronal identity, we immunostained neurons transfected miR-140-3p-Sponge constructs in vivo at E14.5 for Cux1 (Fig. 16B). As illustrated in Fig. 16C, $88.0 \pm 2.2 \%$ of miR-140-3pSponge expressing neurons in bins 3 and 4 were positive for Cux1. On the whole, we concluded that aberrant distribution of miR-140-3p KD neurons is not caused by misregulation of neuronal identity, but is rather due to morphological defects in neurons that hamper their migration during cortical development (Fig. 14C, and D; Fig 15C, and D). 


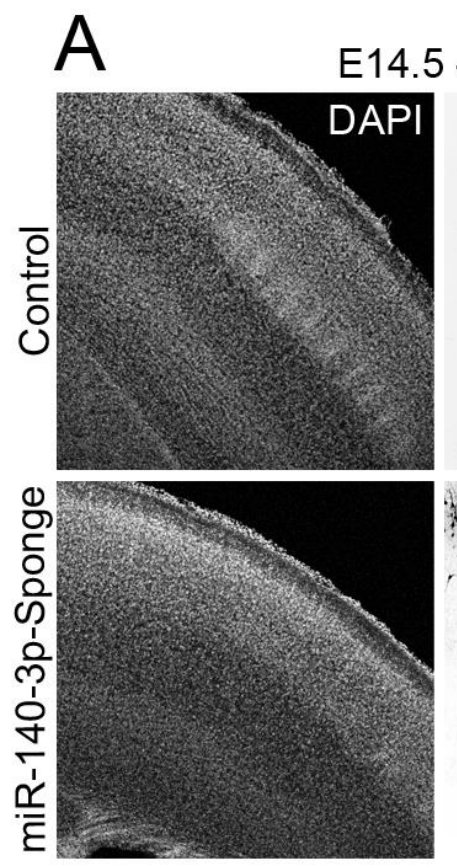

\section{B}

Venus
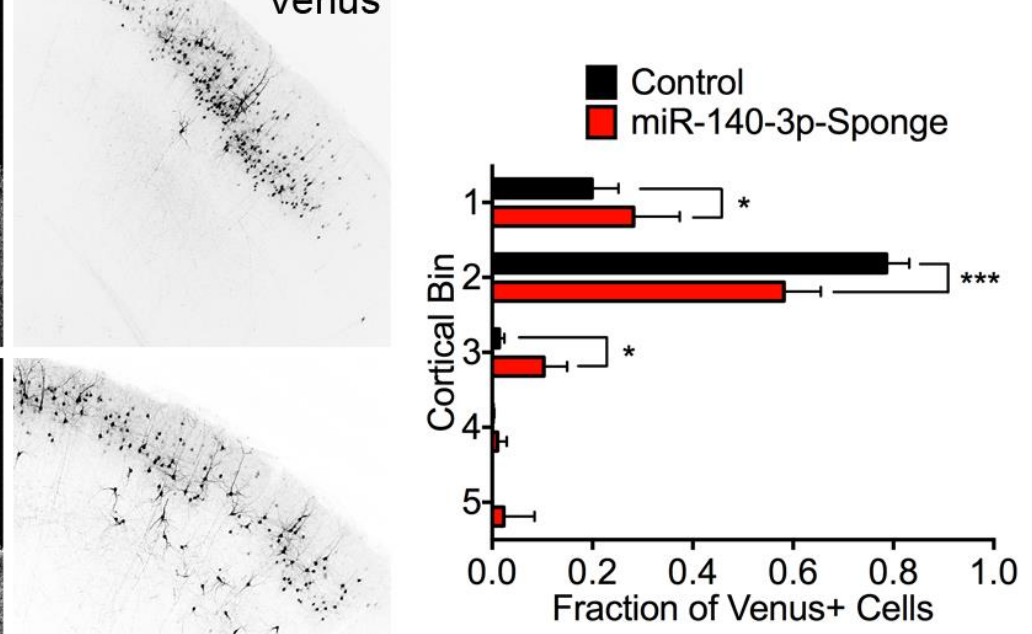

$100 \bar{\mu} \mathrm{m}$

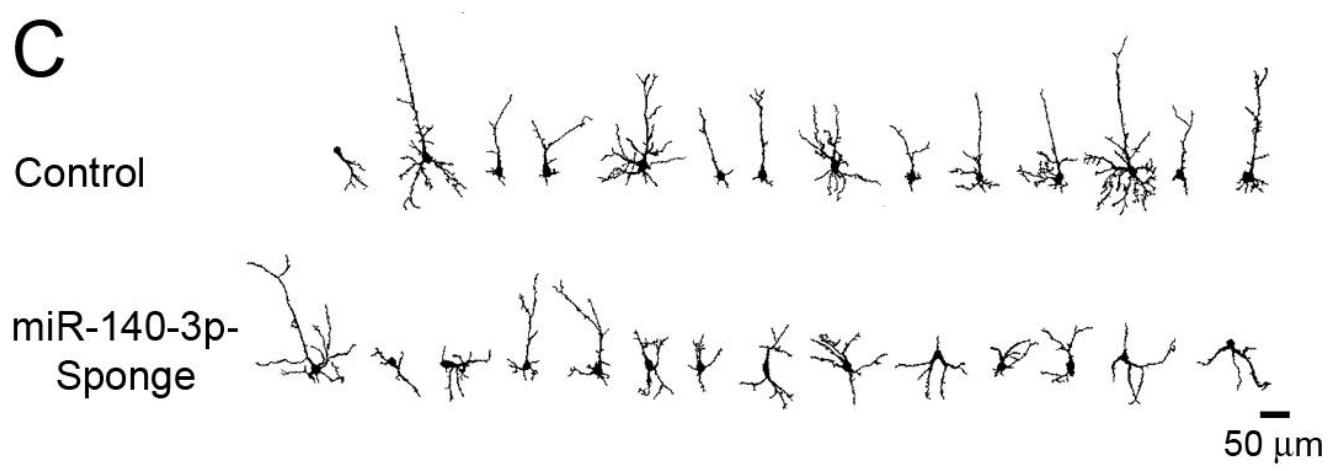

\section{$D \square$ miR-140-3p-Sponge}

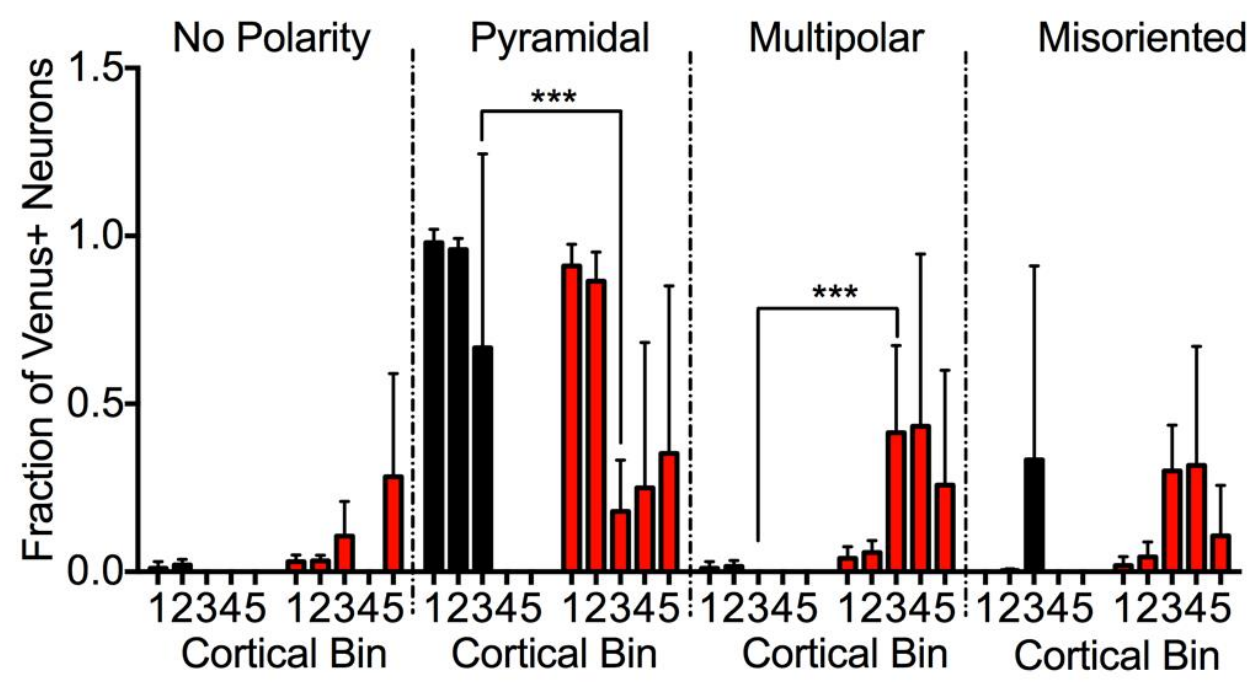

Figure 15. Knock-down of miR-140-3p leads to migratory defects and persistent polarity disruption in cortical neurons in vivo. (A) Representative images of DAPI staining (left panels), and Venus fluorescence (right panels) in P10 cortices of wild type mice. Neuronal progenitors of the cortex were electroporated in utero at E14.5 with plasmids encoding Venus and control sponge (upper panel), or Venus and miR-140-3p sponge (lower 
panel). Scale bar, $100 \mu \mathrm{m}$. (B) Neuronal distribution upon miR-140-3p KD in vivo. Results are represented as averages \pm S.D. Control, $n=5$ brains; miR-140-3p-Sponge, $n=8$ brains; $* * * \mathrm{p}<0.001, * \mathrm{p}<0.05$, two-way ANOVA with Bonferroni post-hoc test. (C) Tracings of representative control and miR-140-3p KD neurons in bins 3 and 4 in P10 brains. Scale bar, $50 \mu \mathrm{m}$. (D) Neuronal polarity classification upon miR-140-3p KD. Results are represented as averages \pm S.D. Control, $\mathrm{n}=5$; miR-140-3p-Sponge, $\mathrm{n}=8$; *** $\mathrm{p}<0.001$, two-way ANOVA with Bonferroni post-hoc test.
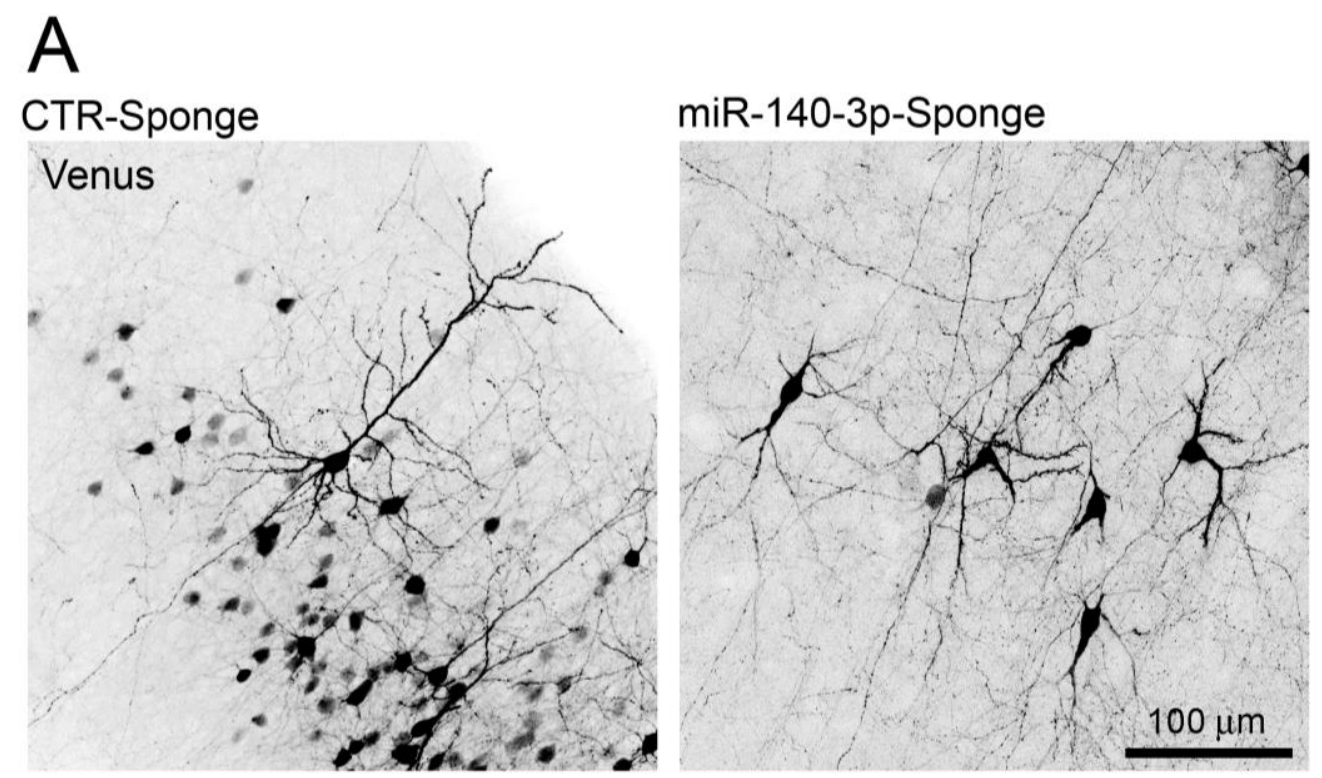

\section{B}

miR-140-3p-Sponge
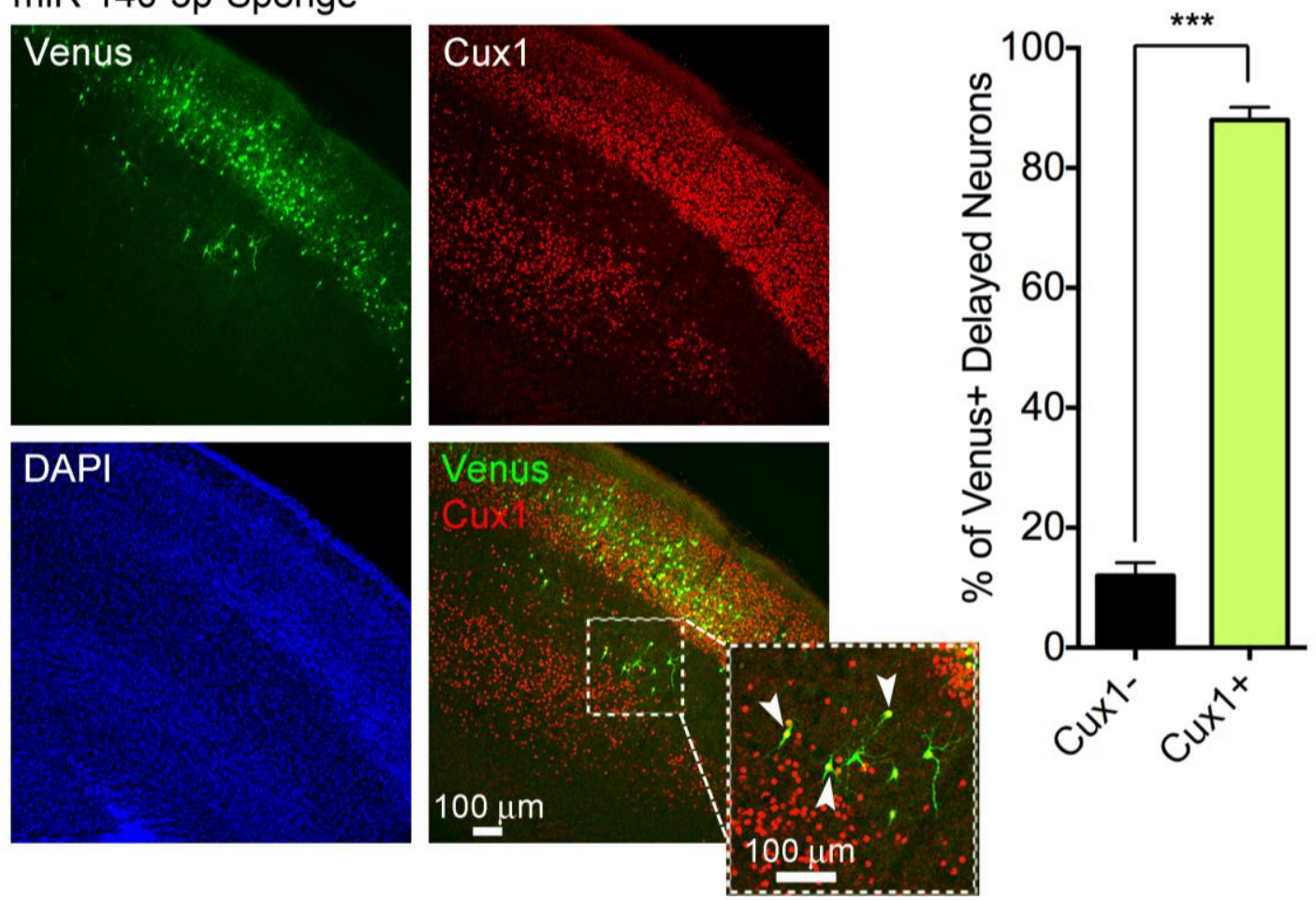

Figure 16. Cortical neurons expressing miR-140-3p display aberrant polarity and express markers of upper cortical layer identity. (A) Example images of Venus 
fluorescence in neurons transfected with control (left panel), or miR-140-3p sponge (right panel). Scale bar, $100 \mu \mathrm{m}$. (B) Cux1 immunostaining of neurons transfected with miR-140$3 p$ sponge. (C) Quantification of relative number of Venus-positive, miR-140-3p sponge transfected and Cux1-positive, or negative neurons in bin 3 and 4. Results are represented as averages \pm S.D. miR-140-3p-Sponge, $\mathrm{n}=3$ brains; $\mathrm{n}=41$ neurons; $* * * \mathrm{p}<0.001$, unpaired t-test.

\subsubsection{Overexpression of miR-140 in primary hippocampal neurons abrogates axon formation}

In order to study if overexpression of miR-140 exerts opposite effects on axon formation as compared to $m i R-140 \mathrm{KO}$ or miR-140-3p KD, we designed and cloned a plasmid expressing tandem repeat of pri-miR-140 (Fig. 17A). Almost half (47\%) of primary hippocampal neurons overexpressing pri-miR-140 (miR-140 OE) failed to project SMI312-positive, and MAP2-negative axons, while $80 \%$ of neurons expressing control plasmid were endowed with a single axon (Fig. 17B). This indicates miR-140-mediated gene expression regulation of axon specification is dose-dependent, namely its depletion leads to formation of multiple axons, and its overexpression abolishes axon formation in neurons. 


\section{A}

a. Control
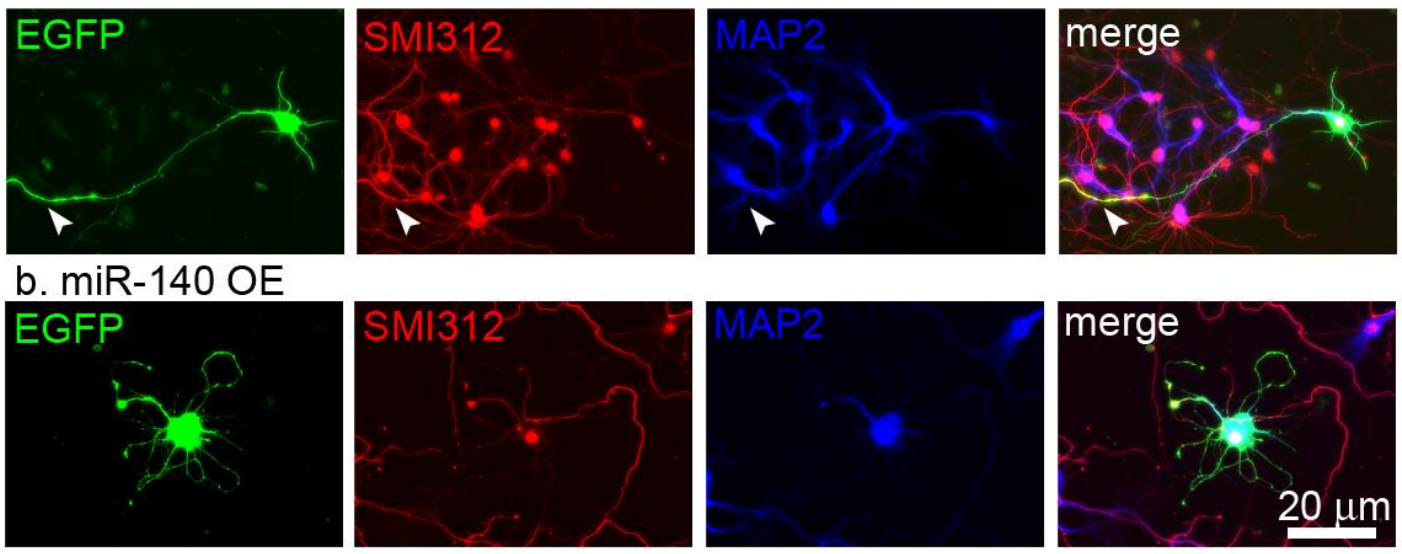

B
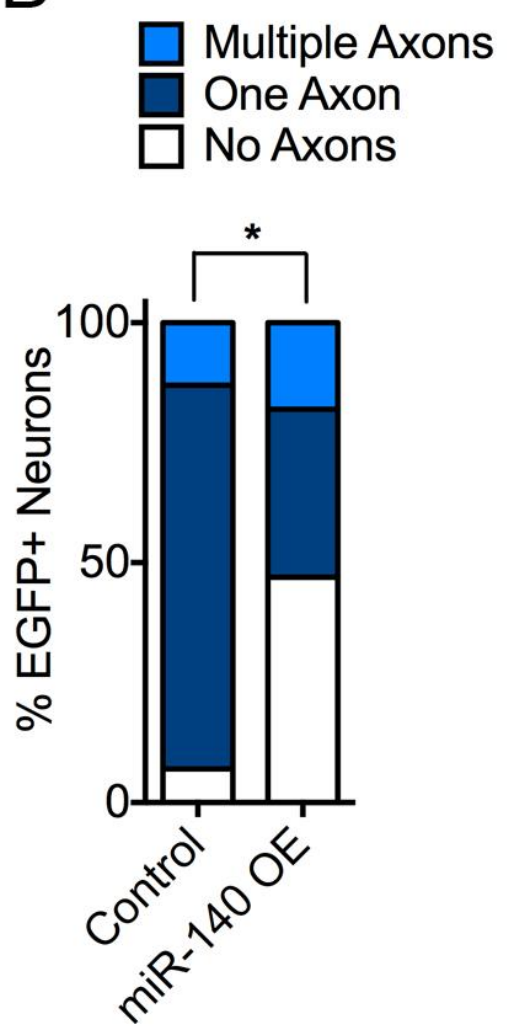

Figure 17. Overexpression of miR-140 abolishes axon formation in primary hippocampal neurons. (A) Nerve cells were transfected with expression plasmid encoding EGFP as a control (a) or EGFP, and a tandemly repeated precursor sequence of miR-140 (miR-140 OE) (b), and fixed at DIV7. Scale bar, $20 \mu \mathrm{m}$. (B) Quantification of polarities in miR-140 overexpressing neurons. Control, $\mathrm{n}=13$; miR-140 OE, $\mathrm{n}=17$ neurons; $* \mathrm{p}<0.05$ by Chi-square test, and $* \mathrm{p}<0.05$, Fisher's exact test between wild type neurons expressing EGFP (a), and neurons overexpressing miR-140 (b). 


\subsubsection{Detrimental effects of $m i R-140$ and $W w p 1 / W w p 2$ loss in neurons do not seem}

to depend on reciprocal miR-host gene interaction

Genetic depletion of $m i R-140$ requires excision of part of $W w p 2$ intronic DNA [201]. This in turn may lead to aberrant pre-mRNA splicing of Wwp2 transcript followed by frame shift or destabilization of pre-mRNA. Additionally, transcription of $W w p 1$ and $W w p 2$ might be compromised by the loss of miR-140, which has been reported to target mRNAs of several transcription factors, like FoxP2, K1f9, and Sox2 [269-271]. Described neuronal effects of miR-140 loss may therefore be attributable to the downregulation of Wwp1 and Wwp2. In order to study if miR-140 depletion from the genome results in changes of Wwp1 and Wwp2 levels, we performed Western blotting in $m i R-140^{+/+}$and $m i R-140^{-/-}$mouse brains using antibodies specific for both Wwp1 and Wwp2 ligases (Fig. 18A). No changes of Wwp ligases levels were detected in brains devoid of miR-140 (Fig. 18B, and 18C).

Alternatively, Cre-mediated critical excision of critical exon in Wwp2 may prompt degradation of its altered mRNA and result in loss or downregulation of miR-140. To corroborate this hypothesis, we performed qRT-PCR to detect both miR-140 strands using P0 brains of $W w p 2^{+/+}$and $W w p 2^{-/-}$mice. Similarly, we observed no alterations in miR-140 levels in $W w p 2^{-/-}$animals (Fig. 18D, and Fig. 18E).

Altogether, it seems that miR-140- and Wwp-mediated regulation of neuronal development present two independent molecular pathways that do not implement reciprocal miR-host gene interactions. 

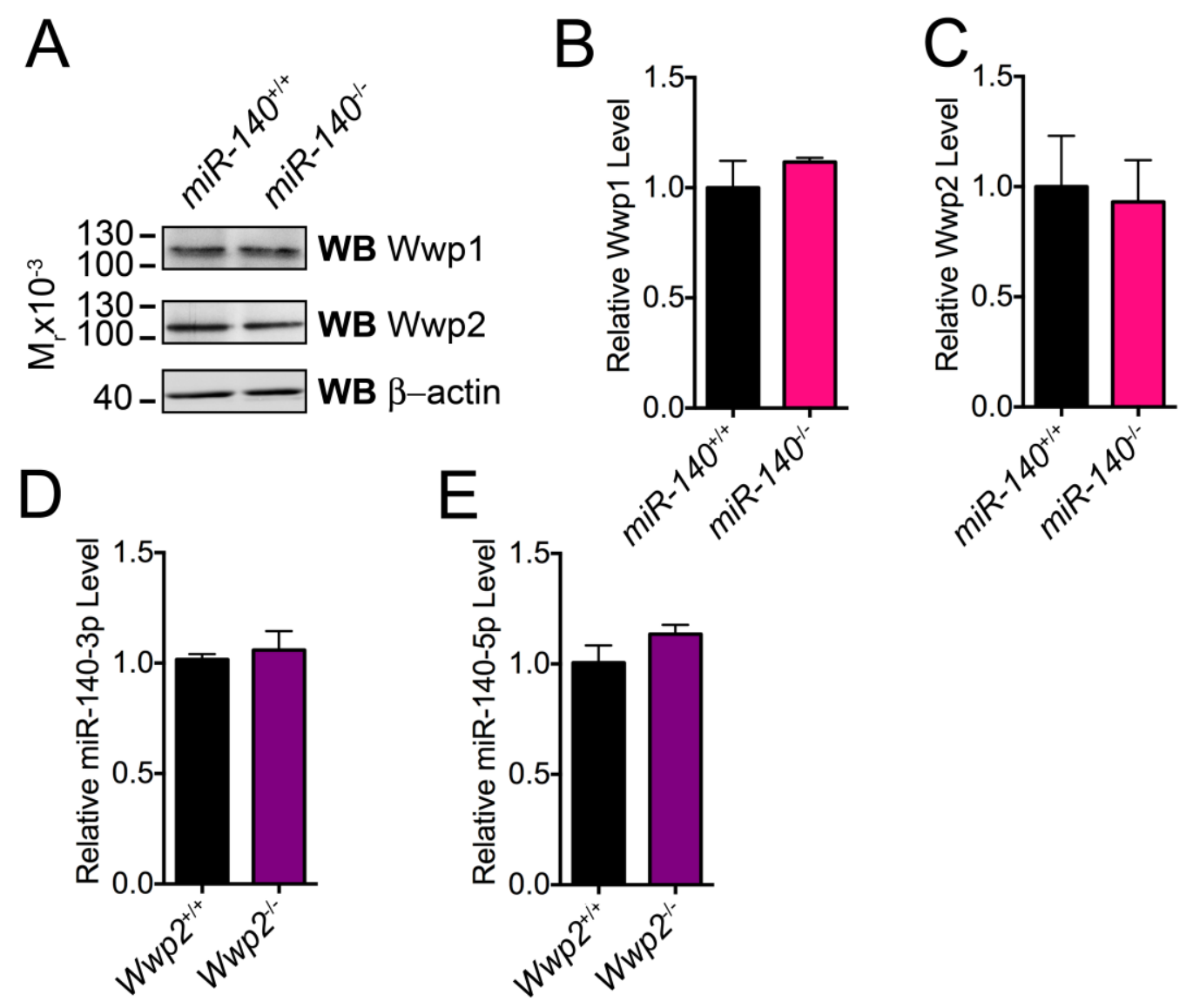

Figure 18. Reciprocal regulation of miR-140 and Wwp ligases. (A) Quantitative Western blotting of the levels of Wwp1 and Wwp2 in cortices of $m i R-140^{+/+}(\mathrm{WT})$ and $m i R-140^{-/-}$ (KO) P0 mice. (B, and C) Wwp1 and Wwp2 protein levels were normalized to $\beta$-actin, and expressed relative to the level of each ligase in $m i R-140^{+/+}$cortex. Results are represented as averages \pm S.D. Control, $m i R-140^{+/+}, \mathrm{n}=4 ; m i R-140^{-/-}, \mathrm{n}=3$; unpaired t-test. (D, and $\mathrm{E}$ ) qRT-PCR for of $-3 p(D)$, and $-5 p(E)$ miR-140 strands in P0 cortices of $W w p 2^{+/+}$, and $W w p 2^{-}$ ${ }^{-}$mice. $W w p 2^{+/+}, \mathrm{n}=3 ; W w p 2^{-/}, \mathrm{n}=2$; unpaired t-test.

\subsubsection{Proteomic screens for putative miR-140 targets involved in regulation of neuronal polarity acquisition}

microRNA exerts several effects on its target mRNA it associates with. Most commonly known effects include recruitment of decapping enzymes, endo- and exonucleases that affect mRNA stability, inhibition of translation initiation or repression of translation elongation [272]. Consequently, knockout of a given microRNA results in slight up-regulation of protein expression of its targets [273]. To identify downstream molecular components of miR-140-mediated regulation of axon formation and neuronal morphology and distribution, we applied a mass spectrometric approach using synaptosomal fractions from $m i R-140^{+/+}$ 
and $m i R-140^{-/-}$mouse cortices (Fig. 19A). Homogenized mouse cortex were subjected to centrifugation in a sucrose gradient. Synaptosome fractions (P2C) collected from the interphase between $1.0 \mathrm{M}$ and $1.2 \mathrm{M}$ sucrose from 5 cortices of $m i R-140^{+/+}$and 5 of the $m i R$ $140^{-/-}$mice were subjected to label-free comparative mass spectrometry (with Olaf Jahn, MPIem, Göttingen). Next, we validated the purification procedure by Western blotting in protein fractions obtained along the procedure. PSD-95, a marker of postsynaptic density (PSD), was consequently enriched in $\mathrm{P} 2 \mathrm{C}$, which was paralleled by persistent level of cytoplasmic Rab-GDI (Fig. 19B). Based on the number and amount of each unique peptide detected by mass spectrometry, we computed p-values of detection with Bonferroni posthoc test. Putative miR-140 target mRNAs were chosen based on fold increase in miR-140 KO synaptosomes ( $\log 2$ ratio WT/KO), p-value, and Bonferroni post-hoc test (Fig. 19C). To exclude indirect effects of miR-140 depletion, we focused on mRNAs with putative miR140-3p binding sites in 3' untranslated regions (3'UTRs). PITA algorithm, allowing for calculation of Gibbs free energy changes upon microRNA-mRNA binding, revealed candidate miR-140-3p binding sites in 3'UTRs of Nefm and Fyn mRNAs (asterisks, Fig. 19D). We detected one sequence in Nefm, and five in Fyn 3'UTRs putatively bound by miR$140-3 p$. We then validated the results of proteomics results by Western blotting in synaptosomal fractions of $m i R-140^{+/+}$(WT) and $m i R-140^{-/-}$(KO) mouse brains, using antibodies against neurofilament M (NFM, encoded by Nefm gene) and Fyn kinase (encoded by Fyn gene) (Fig. 19E). Quantification of relative levels of NFM and Fyn revealed a trend towards a slight up-regulation of both proteins in synaptosomal fraction of miR-140 KO brains (NFM, $10.5 \pm 26.8 \%$; Fyn, $22.4 \pm 23.0 \%$ increase in $m i R-140 \mathrm{KO}$ ). 
A

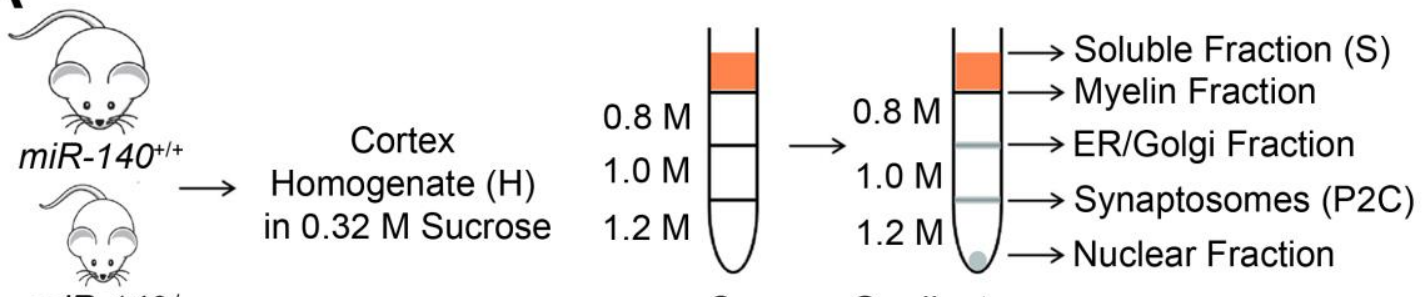

$\operatorname{miR}-140^{-1-}$

Sucrose Gradient

B

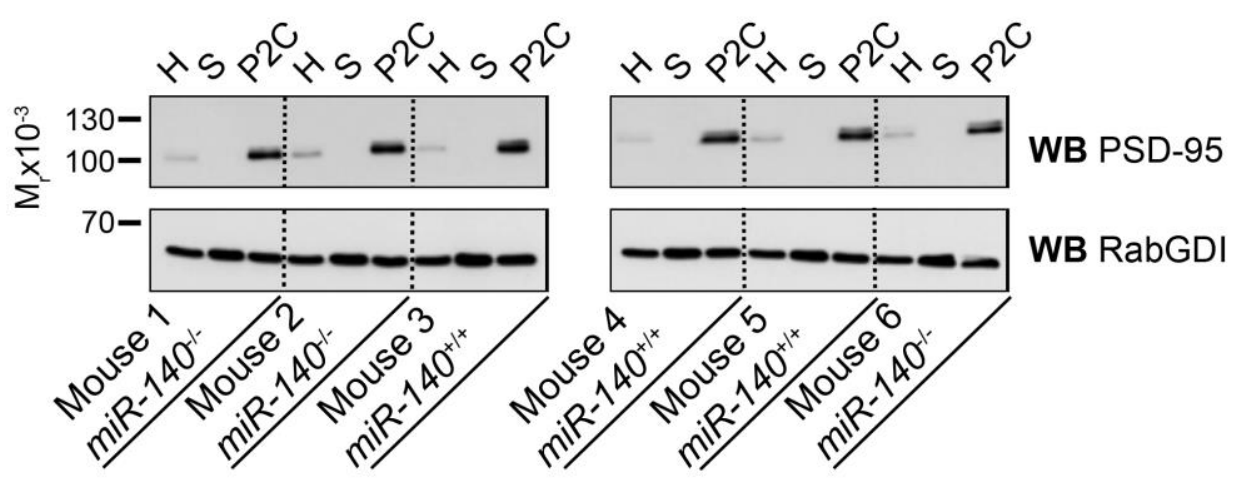

\begin{tabular}{ccc}
$C_{\begin{array}{c}\text { Gene } \\
\text { Name }\end{array}}$ & $\begin{array}{c}\text { UniProt } \\
\text { Accession }\end{array}$ & $\begin{array}{c}\text { Log2 ratio } \\
\text { WT/KO }\end{array}$ \\
\hline Mapk3 & Q63844 & -0.50 \\
Prkar1b & P12849 & -0.50 \\
Phactr1 & Q2M3X8 & -0.42 \\
* Nefm & P08553 & -0.40 \\
Ptprs & B0V2N1 & -0.30 \\
Wasf1 & Q8R5H6 & -0.25 \\
* Fyn & P39688 & -0.23 \\
\hline
\end{tabular}

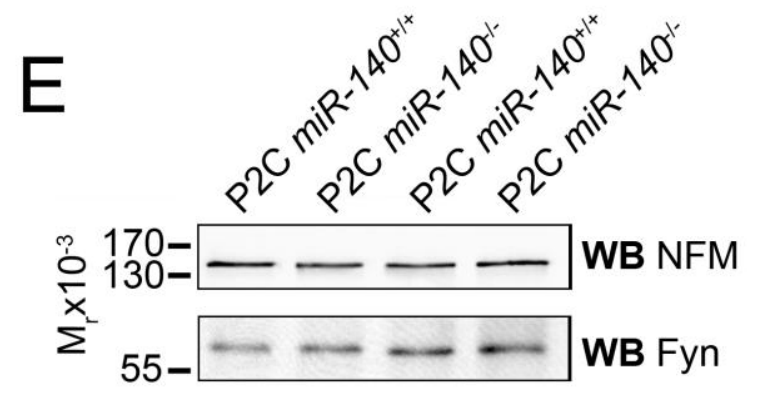

D

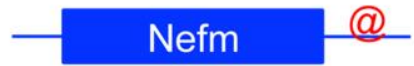

Fyn @@@@
300bp

@ Putative miR-140-3p Binding Sites

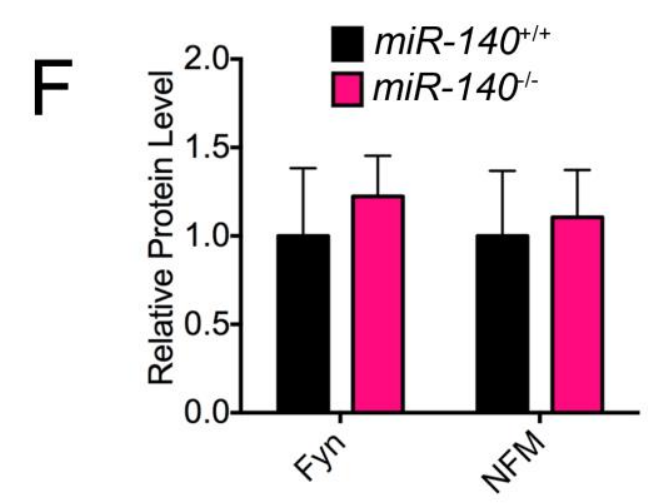

Figure 19. Identification of miR-140 downstream targets. (A) A schematic of synaptosome purification from $m i R-140^{+/+}$and $m i R-140^{-/-}$mouse brains. (B) Validation of synaptosomal preparation by Western blotting. (C) List of selected putative miR-140 targets identified by quantitative proteomics. Asterisks depict proteins possessing putative miR140-3p binding sites along their 3' UTR according to PITA free Gibbs energy calculation 
algorithm. (D) Schematic of mRNAs of Nefm and Fyn with putative miR-140 binding sites in their 3'UTR (@). Scale bar, 300 base pairs. (E) Validation of the proteomics experiment by Western blotting with antibodies against NFM (upper panel), and Fyn (lower panel) in synaptosomal fractions from $m i R-140^{+/+}$and $m i R-140^{-/-}$cortices. (F) Quantification of Western blotting experiment in (E). The level of NFM, and Fyn was normalized to protein loading and expressed relative to the level in $m i R-140^{+/+}$. Results are represented as averages \pm S.D. $m i R-140^{+/+}, \mathrm{n}=5 ; m i R-140^{-/}, \mathrm{n}=5$, unpaired t-test.

\subsubsection{Sox9 is expressed in postmitotic neurons of the murine central nervous system}

We revealed that Wwp ligases alongside miR-140 are indispensable for proper neuronal distribution in the cortex and control neuronal polarity acquisition in primary hippocampal nerve cells and cortical neurons in vivo. Concomitant expression patterns of $W w p 2$ and $m i R$ 140 have been reported in non-neuronal tissue by several independent groups $[172,258,274]$. Interestingly, both genes seem to be the major targets of Sox 9 transcription factor in cartilage [258]. Sox9 has been previously reported as a regulator of differentiation expressed in radial glial cells, chondroprogenitor cells, and cardiac mesenchymal cells, where it plays a crucial roles in gliogenesis [275], chondrocyte differentiation [276], and endothelial-mesenchymal transition [277]. Interestingly, developmental profiling of Sox9, Wwp1, and Wwp2 mRNAs isolated from developing and adult murine cortex reveals expression of Sox9 in adult murine brain and striking resemblance of $\operatorname{Sox} 9$ and $W w p 2$ expression patterns (Fig. 20A). Moreover, the Sox 9 protein level mirrors the amount of Wwp2 in cortical homogenates prepared from mice at different developmental stages (Fig. 20B), while the level of Wwp1 seem to be inversely correlated to Sox 9 and Wwp2. This indicates that Sox 9 may also serve as the major transcriptional regulator of Wwp2 in the brain, and may regulate Wwp2/miR-140 duo in neurons. Next, we sought the Sox9 expression in postmitotic nerve cells. We stained the coronal sections of P0 mouse cortex with antibodies against Sox9 and neuronal identity marker Ctip2 (COUP-TF interacting protein 2) [278]. Intriguingly, we observed extensive Sox9 immunostaining signals in hippocampal and cortical neuronal nuclei positive for Ctip2. To then further corroborate the specificity of Sox9 labeling, we immunolabeled P10 brain sections of $\operatorname{Sox} 9^{\mathrm{f} / \mathrm{f}}$ mice after in utero electroporation at E14.5 with plasmid encoding GFP (control) or two plasmids, each for GFP and Cre recombinase (Sox9 KO) with Sox9 antibody used previously (Fig. 20 C), and NeuN to distinguish neuronal nuclei (Fig. 20D). Sox9 labeling signal was pronounced in control neurons, but unambiguously absent in $\operatorname{Sox} 9 \mathrm{KO}$ 
nerve cells. Immunolabeling intensity was further measured on manually selected somata with ImageJ software to elucidate almost complete loss of staining in Sox9 KO neurons $\left(\operatorname{Sox} 9^{\mathrm{f} / \mathrm{f}}+\mathrm{GFP} 1 \pm 0.21 ; \operatorname{Sox} 9^{\mathrm{f} / \mathrm{f}}+\mathrm{GFP}+\right.$ iCre $\left.0.05 \pm 0.21\right)$ (Fig. 20E). Taken together, Sox 9 is expressed in postmitotic neurons of the cortex, and its expression pattern strongly resembles the one of Wwp2.

\subsubsection{Sox9 regulates axon specification in postmitotic primary hippocampal neurons}

To corroborate if Sox9 acts along with Wwp2 and miR-140 in the regulation of axon acquisition, we performed Sox $9 \mathrm{KD}$ and overexpression study in primary hippocampal neurons (Fig. 21A) by transfecting sh-RNA expression vectors encoding Venus, or cotransfecting EGFP and Sox9 expression vectors to wild type neurons. Sox $9 \mathrm{KD}$ resulted in the emergence of neurons projecting multiple axons (Fig. 21A, a, and b). While only about $10 \%$ of control neurons projected multiple axons, $38 \%$ of nerve cells transfected with Sox 9 KD plasmid displayed more than a single axon (Fig. 21B a, and b). Conversely, overexpression of Sox9 in nerve cells, like overexpression of miR-140, leads to loss of axon specification (Fig. 21A, c, and d). Moreover, $88 \%$ of neurons presented a single axon whilst $23 \%$ of Sox 9 overexpressing neurons failed to specify an axon, characterized by pronounced SMI312 signals and absent MAP2 labeling (Fig. 21B c, and d). Given that primary neurons were prepared form P0 hippocampi, when the neurogenesis of glutamatergic neurons had been completed $[279,280]$, we concluded that Sox9 plays an important role in axon specification in postmitotic primary hippocampal neurons. 

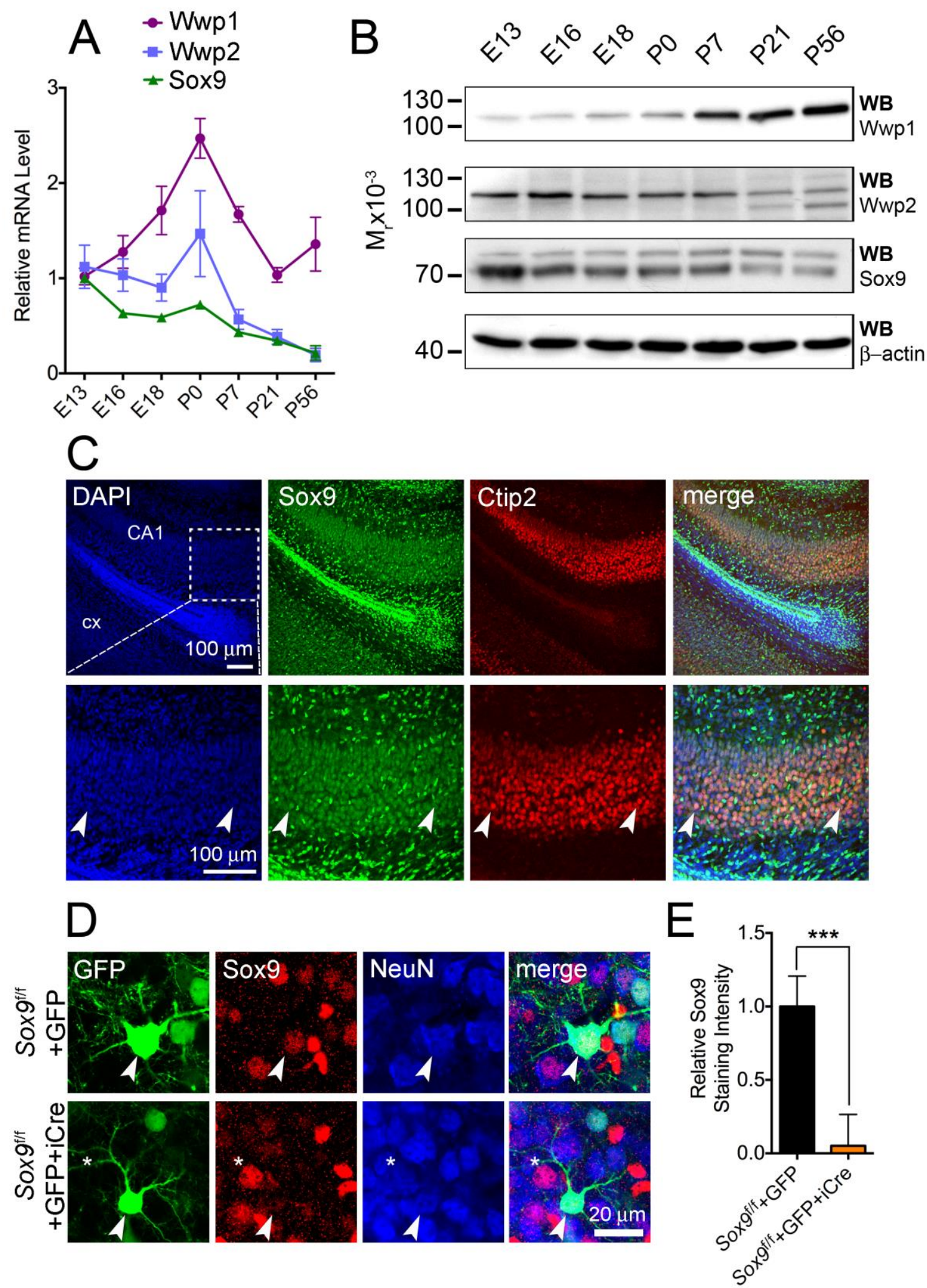

Figure 20. Sox9 expression in postmitotic neurons of the murine central nervous system. (A) Developmental expression profiling of Wwp1, Wwp2, and Sox 9 mRNAs by qRT-PCR in murine cortices of different developmental stages. Results are represented as averages \pm SEM. Transcript level of each gene was normalized to housekeeping Gapdh, and Oaz1 at each developmental time point. (B) Western blotting in cortical lysates of mice of 
depicted age using antibodies against Wwp1 (upper panel), Wwp2 (second from top), Sox9 (second from bottom), and $\beta$-actin (bottom panel). (C) Immunostaining in coronal sections of P0 wild type mouse brain using DAPI (leftmost panels), and antibodies against Sox9 (second panel from the left), and neuronal marker Ctip2 (third panels from the left), CA1, hippocampal subfield CA1; cx, cortex. Scale bar, $100 \mu \mathrm{m}$. Arrowheads on the bottom panels point to Sox9-, and Cux1- positive neuronal nuclei in the CA1 field of mouse hippocampus. (D) Expression of Sox 9 in postmitotic neurons. Sox $9^{\mathrm{f} / \mathrm{f}}$ neurons were electroporated in utero with GFP (control, top panel), and GFP and Cre recombinase expressing plasmids (Sox 9 knockout, KO, bottom panel) at E14.5. Brains at P10 were immunostained against Sox 9, and NeuN. Note the prominent signal of Sox9 staining in the nuclei of control cells (arrowheads, top panel), and loss thereof in Cre-expressing, Sox9 KO neurons (bottom panel). Asterisk on the bottom panel indicates Sox9-positive nucleus of a neuron not transfected with Cre plasmid. (E) Quantification of immunostaining signal intensity in neurons expressing GFP or GFP and Cre recombinase. Results are represented as averages \pm S.D. Control, Sox $9^{\mathrm{f} / \mathrm{f}}+$ GFP, $\mathrm{n}=6$ neurons; KO, Sox $9^{\mathrm{f} / \mathrm{f}}+\mathrm{GFP}+\mathrm{iCre}, \mathrm{n}=5$ neurons. Note the almost complete loss of Sox 9 staining in Sox $9 \mathrm{KO}$ neurons. Sox $9^{\mathrm{f} / \mathrm{f}}$ mouse brains, after in utero electroporation, were provided by Dr. Mikio Hoshino (National Institute of Neuroscience, Japan). ${ }^{* * *} \mathrm{p}<$ 0.001 , unapired t-test.

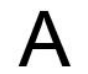

a. Control
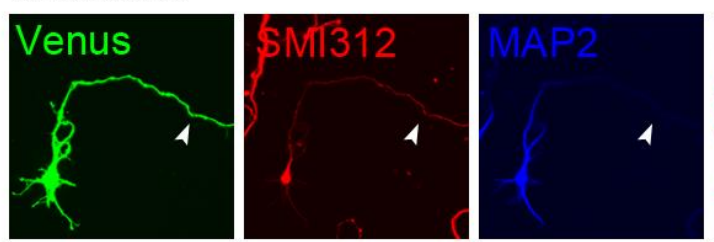

b. Sox9 KD

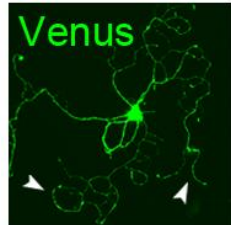

c. Control
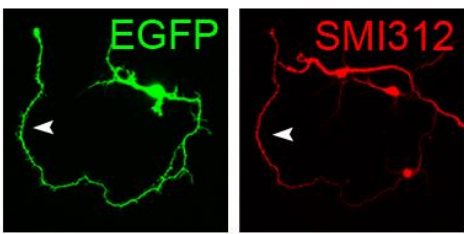

\section{d. Sox9 OE}

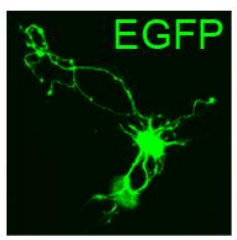

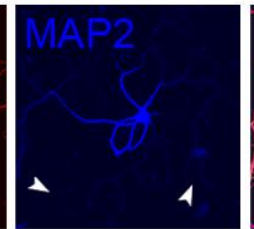

1
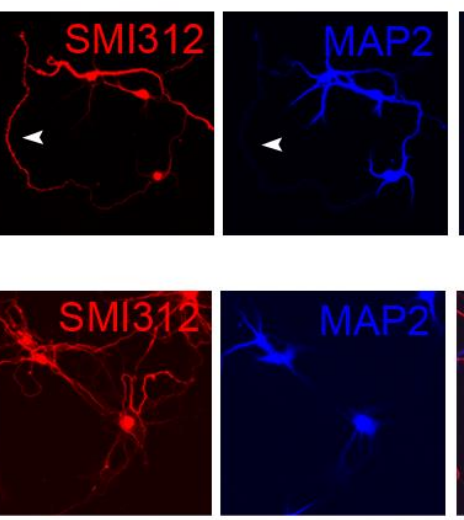
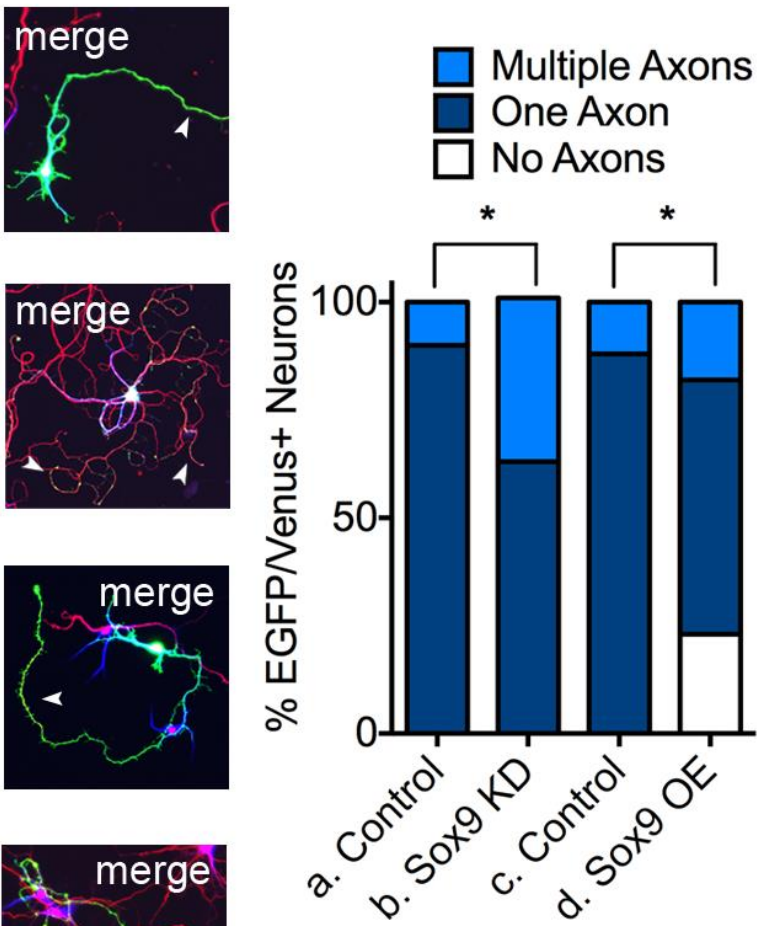

Figure 21. Sox9 regulates axon acquisition in primary hippocampal neurons. (A) Representative images of wild type primary hippocampal neurons. Nerve cells were transfected at DIV1 with plasmid encoding non-silencing sh-RNA (a; control), or sh-RNA 
to downregulate Sox9 (b; Sox9 KD), or EGFP (c; control), or co-transfected with EGFP, and expression vector for Sox9 (d; Sox9 OE), fixed at DIV7 and immunostained for SMI312 and MAP2. Scale bar, $20 \mu \mathrm{m}$. (B) Quantification of number of axons projected by a single neuron. Control (a), $n=30$ neurons; Sox9 KD (b), $n=32$ neurons; control (c), $n=17$; Sox 9 OE (d), $\mathrm{n}=31$ neurons; $* * * * \mathrm{p}<0.0001$ by Chi-square test, and $* \mathrm{p}<0.05$, Fisher's exact test between wild type neurons expressing control sh-RNA (a), and Sox9 KD neurons (b); * $\mathrm{p}<0.05$, Fisher's exact test between control neurons (c), and Sox9 OE neurons (d).

\subsubsection{Sox9 emerges as a potent regulator of neuronal distribution an a regulator of cortical neuron polarity formation}

To study if loss of Sox9 poses detrimental effects on postmitotic neurons, we studied distribution, and morphology of cortical neurons after in utero electroporation. We transfected the pool of cortical progenitors at E14.5 with a plasmid encoding for nonsilencing sh-RNA (control), or sh-RNA against Sox9 (Sox9 KD) (Fig. 22A). Quantitative analysis of neuronal distribution revealed statistically significant differences in the number of cells located in bin 1. While most of control neurons $(72.7 \pm 4.8 \%)$ distributed in bin 1 , only $47.8 \pm 5.1 \%$ of Sox 9 KD neurons were counted there. Moreover, in deep cortical layers, we observed hardly any control cells (bin 3, $0.4 \pm 0.6 \%$; bin 4, $0.2 \pm 0.4 \%$; bin 5, $2.0 \pm 2.2$ $\%$ ), whereas increased fraction of Sox9 KD neurons were found in bin $3(6.9 \pm 2.3 \%)$, bin $4(3.9 \pm 2.7 \%)$, and bin $5(12.3 \pm 11 \%)$ (Fig. 22B). Due to mere control cells in deep cortical layers (bin 3 to bin 5), for morphological studies we split the analyzed cortices in three instead of five bins. Although we detected no statistically significant differences, we observed a clear trend towards an increase in Sox9 KD neurons with multiple apical dendrites in bin 2 and 3 (Fig. 22C, cell 5, 8 from the left, bottom panel, Fig. 22D). 


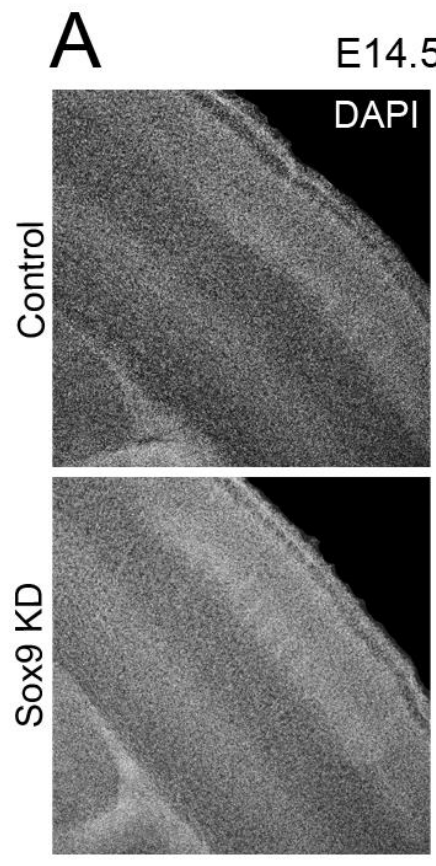

$\mathrm{B}$
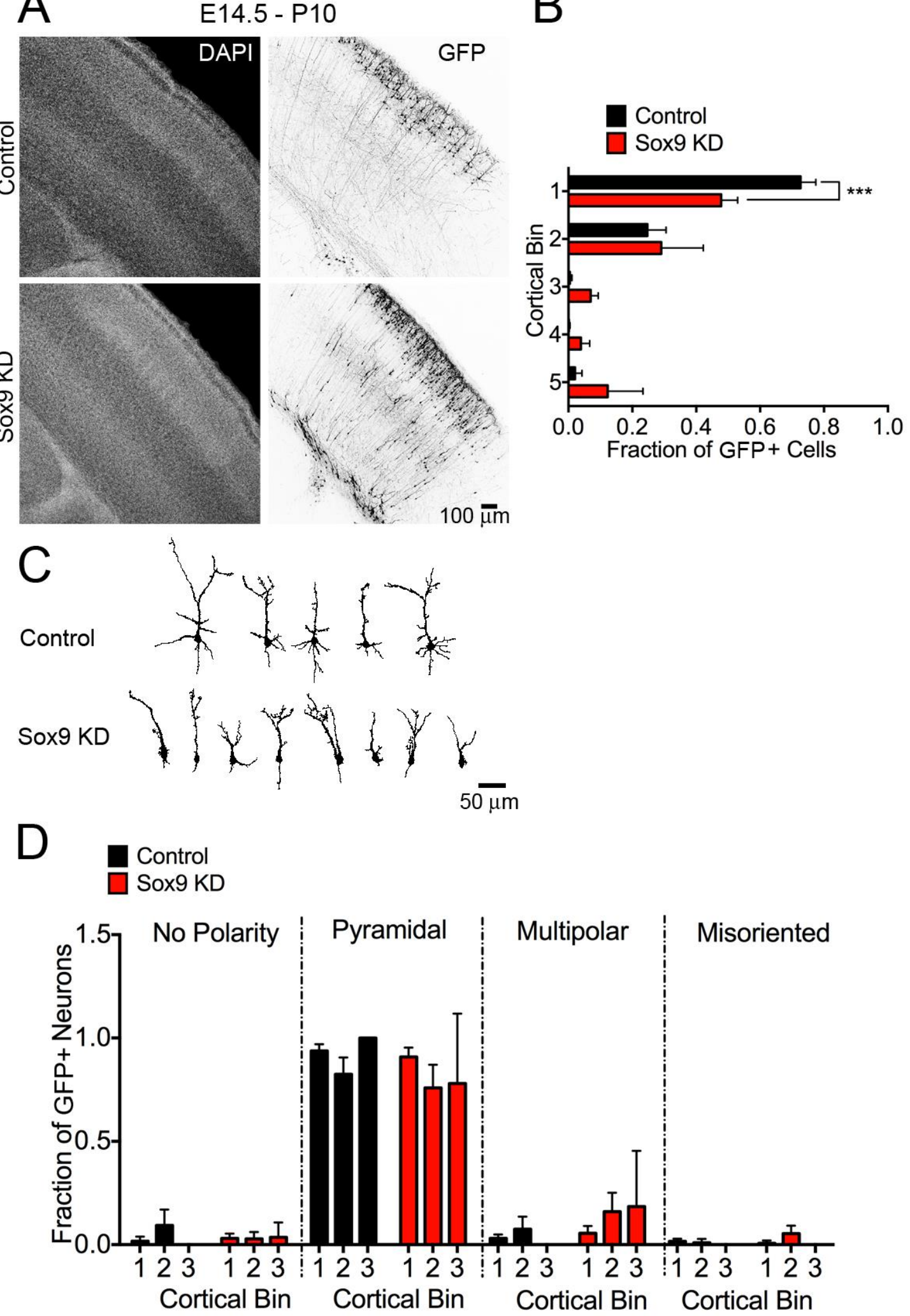

Figure 22. Acute KD of Sox9 alters distribution of cortical neurons in vivo. (A) Representative images of DAPI staining (left panels), and anti-GFP immunolabeling (right panels) in P10 cortices of wild type mice. Cortical progenitors were electroporated in utero at E14.5 with plasmids encoding Venus, and control non-silencing RNA (upper panel) or Venus, and sh-RNA to downregulate Sox 9 (Sox9 KD, lower panel). Scale bar, $100 \mu \mathrm{m}$. (B) 
Neuronal distribution upon Sox9 KD in vivo. Results are represented as averages \pm S.D. Control, $\mathrm{n}=5$ brains; Sox9 KD, $\mathrm{n}=4$ brains; *** $\mathrm{p}<0.001$, two-way ANOVA with Bonferroni post-hoc test. (C) Tracings of representative control, and Sox9 KD neurons in bin 3 of P10 brains. Scale bar, $50 \mu \mathrm{m}$. (D) Morphological classification of neurons upon Sox9 KD. Results are represented as averages \pm S.D. Control, $n=5$ brains; Sox 9 KD, $n=4$ brains; two-way ANOVA with Bonferroni post-hoc test.

Next, we analyzed brains of $\operatorname{Sox} 9^{\mathrm{f} / \mathrm{f}}$ animals, where Sox 9 was depleted from a fraction of cortical progenitors by in utero electroporation with the Cre expression plasmid. Observed neuronal distribution alterations and morphological deficits upon Sox9 knockout (Sox9 KO) from neurons were far more pronounced than those upon Sox9 KD (Fig. 23A). Like in the case of the Sox 9 KD experiment, majority of control (Sox $9^{\mathrm{f} / \mathrm{f}}+$ GFP) pyramidal neurons reached bin $1(77.2 \pm 13.7 \%)$. Additionally, we found only four control neurons among all cortical cross sections analyzed from five P10 brains within bins 3 and 4, and all of them displayed polarized morphology, typical for pyramidal neurons (Fig. 23 C, upper panel; Fig. 23D). On the other hand, only $27.8 \pm 7.4 \%$ of $\operatorname{Sox} 9 \mathrm{KO}$ reached bin 1 , while majority of Sox9 KO nerve cells aberrantly located in bin $2(65.5 \pm 9.9 \%)$, and $5.5 \pm 3.6 \%$ of Sox9 KO neurons were found in bin 3. Additionally, only a minor fraction of $\operatorname{Sox} 9 \mathrm{KO}$ neurons in bin 3 presented normal pyramidal morphology $(6.3 \pm 7.4 \%)$. Nerve cells devoid of Sox 9 in bin 3 did not present a prominent apical dendrite $(17.9 \pm 22.5 \%)$, while $50.8 \pm 3.4 \%$ projected more than one prominent dendrite (Fig. 23C, lower panel, cell 2, 3, 6, 8 from the left), and $25.0 \pm 16.7 \%$ showed a misoriented prominent dendrite, sometimes running parallel to the pial surface or aberrantly projecting towards the corpus callosum (Fig. 23C, lower panel, cell 1, 4, 5, 7 from the left; Fig. 23D). Marked, almost complete loss of pyramidal cell characteristics in Sox 9 KO neurons of bin 3 and 4 makes this genetic manipulation causative of the most pronounced polarity defects we have encountered during this study. Altogether, these data prove that Sox 9 has an important role in shaping neuronal polarity, and regulating migration of developing cortical neurons of layer II/III. Moreover, Sox9, together with Wwp ligases and miR-140 seem to pose synergistic effects on axon-dendrite specification, and neuronal migration. 
A $\mathrm{E} 14.5-\mathrm{P} 10$
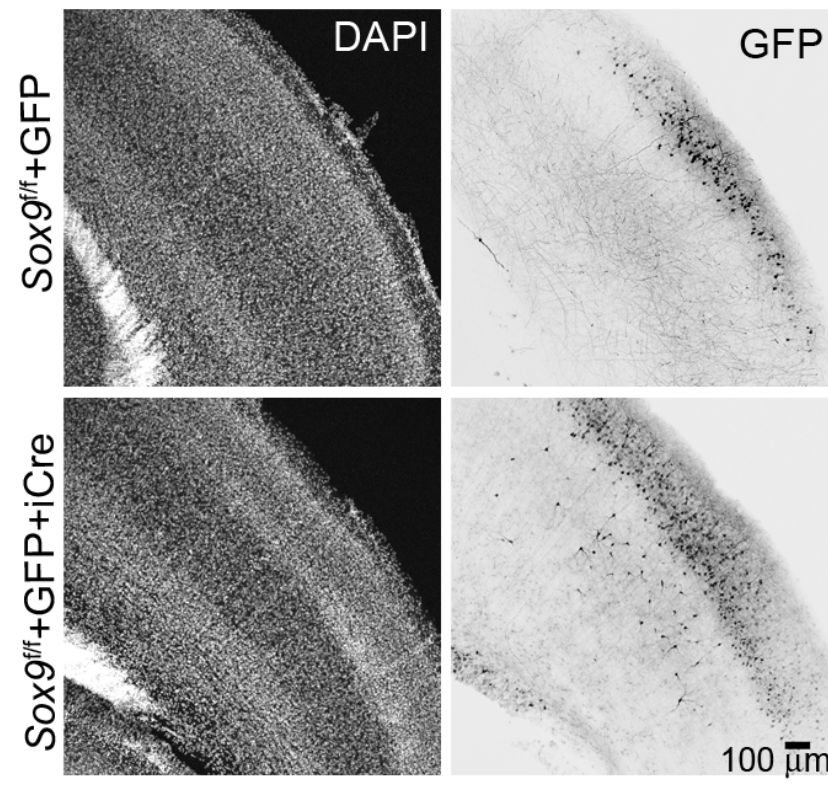

GFP

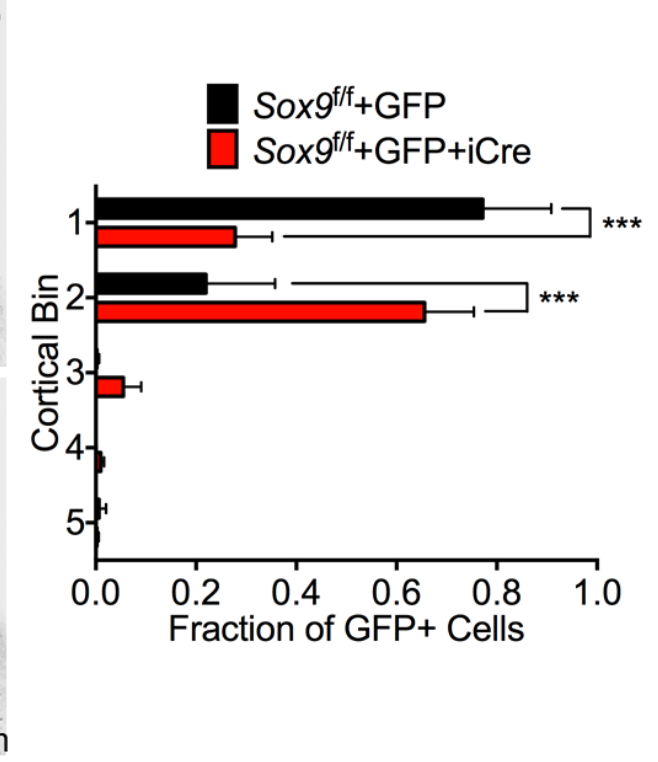

$100 \overline{\mu m}$

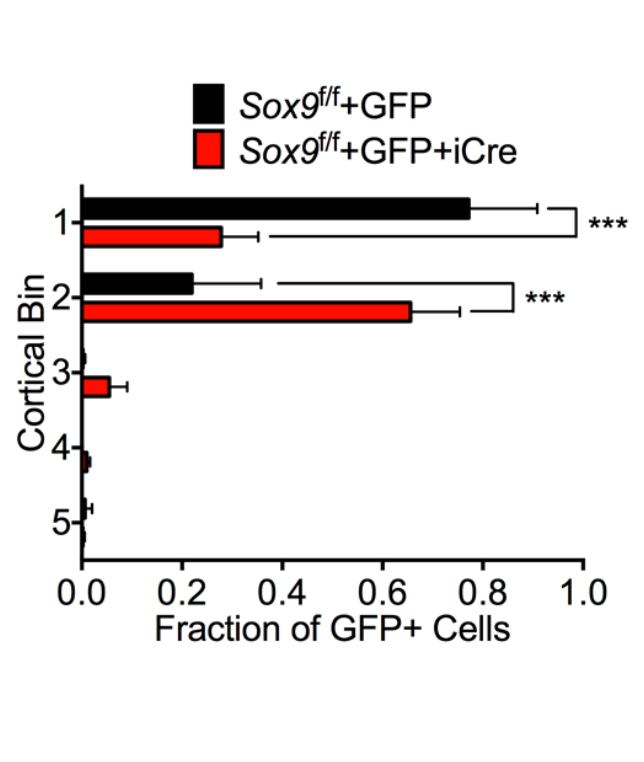

B

C

So $9^{f f}+$ GFP
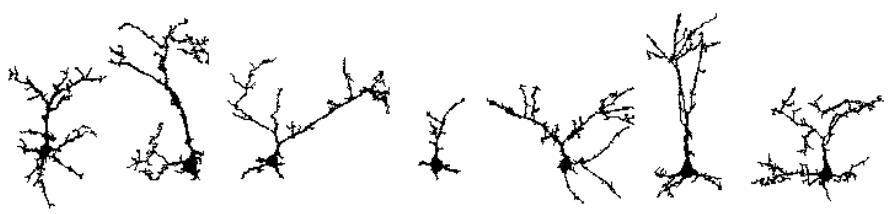

So $\times 9^{f f}+$ GFP + iCre

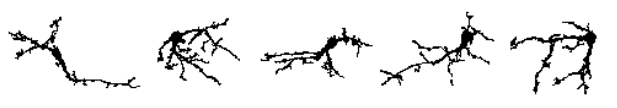

x

D

Soxg $9^{\mathrm{ff}}+\mathrm{GFP}$

Sox $9^{f / f}+$ GFP+iCre

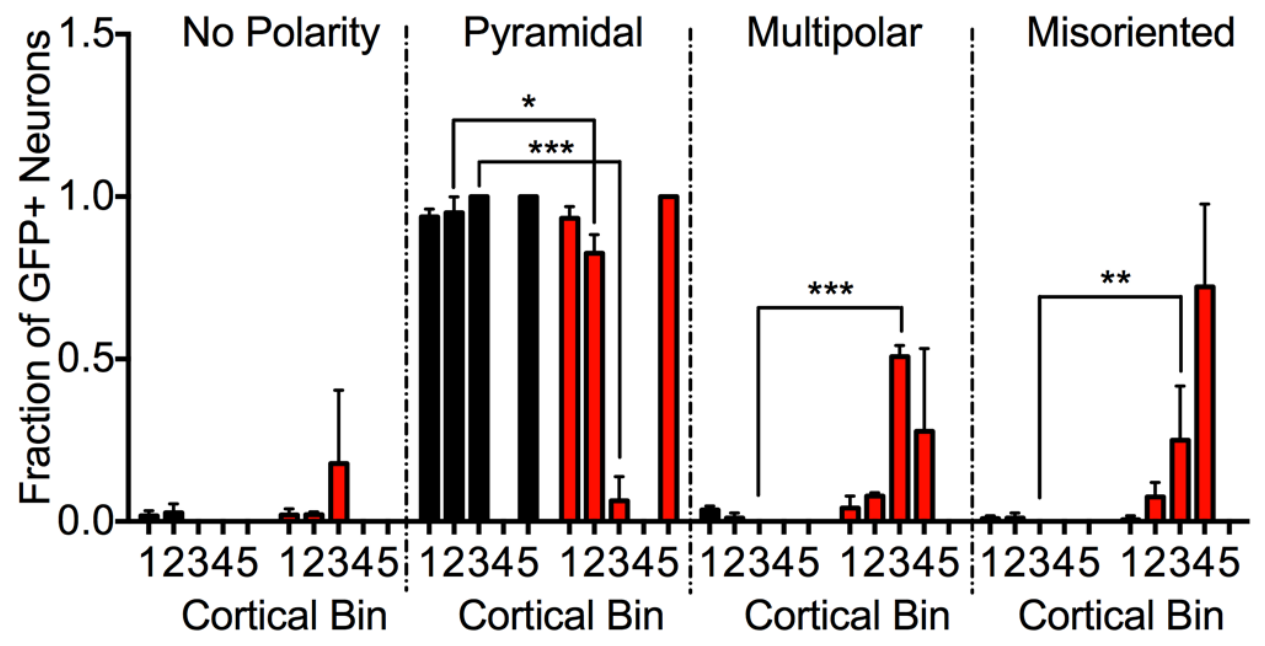

Figure 23. Loss of Sox9 results in severe migratory aberrances and strong polarity defects in cortical neurons in vivo. (A) Representative images of DAPI staining (left panels), and GFP/Venus fluorescence (right panels) in P10 cortices of Sox $9^{\mathrm{f} / \mathrm{f}}$ mice. Neurons were electroporated in utero at E14.5 with plasmids encoding myr-Venus and GFP as a control (upper panel), or myr-Venus, GFP, and Cre recombinase to induce Sox9 knockout 
(Sox9 KO, lower panel). Scale bar, $100 \mu \mathrm{m}$. (B) Quantification of neuronal distribution upon Sox9 KO in vivo. Results are represented as averages \pm S.D. Sox $9^{\mathrm{f} / \mathrm{f}}+$ GFP, $\mathrm{n}=5 ; \operatorname{Sox} 9^{\mathrm{f} / \mathrm{f}}+$ GFP + iCre, $\mathrm{n}=4$ brains; $* * * \mathrm{p}<0.001$, two-way ANOVA with Bonferroni post-hoc test. (C) Tracings of representative control, and Sox9 KO neurons in bin 3 and 4 of P10 brains. Scale bar, $50 \mu \mathrm{m}$. (D) Classification of neuronal polarities upon Sox9 KO. Results are represented as averages \pm S.D. Sox $9^{\mathrm{f} / \mathrm{f}}+$ GFP, $\mathrm{n}=5$; Sox $9^{\mathrm{f} / \mathrm{f}}+\mathrm{GFP}+\mathrm{iCre}, \mathrm{n}=4$ brains. Sox $9^{\mathrm{f} / \mathrm{f}}$ mouse brains after in utero electroporation were provided by Dr. Mikio Hoshino (National Institute of Neuroscience, Japan); ${ }^{* * *} \mathrm{p}<0.001,{ }^{* *} \mathrm{p}<0.01,{ }^{*} \mathrm{p}<0.05$, two-way ANOVA with Bonferroni post-hoc test.

\subsection{HECT ligases of Nedd4 family in mature neurons}

\subsubsection{Wwp ligases control development of dendritic spines}

Notably, Wwp1 and Wwp2 are expressed in the cerebral cortex of mature mice, and protein expression of Wwp1 is developmentally up-regulated (Fig. 20B). Such an expression profile is a characteristic feature of proteins involved in synapse formation and function [281]. Although Wwp2 protein expression decays as the cortical development proceeds, its levels are stable in the mature brain (compare levels of Wwp2 between P21 and P56, Fig. 20B). This indicates that Wwp1 and Wwp2 may play an important role in synaptogenesis, and regulate synaptic transmission in mature neurons. Supportive of this hypothesis are the results of genetic screens in C. elegans demonstrating that mutations in $w w p-1$ (an ortholog of murine $W w p l)$ disrupted the distribution of a synaptic marker protein [282]. These observations illustrate that $w w p-1$ may be implicated in worm synaptogenesis.

To verify if Wwp ligases play roles in synapse formation, we established and analyzed a neuron specific, NEX1-Cre driven conditional double knockout of $W w p 1$, and $W w p 2$ $\left(W w p 1^{\mathrm{f} / \mathrm{f}} ; \quad W w p 2^{\mathrm{f} / \mathrm{f}} ; \quad N E X 1-\mathrm{Cre}^{+/-} ; W w p 1 / 2\right.$ dcKO$)$ [202]. We transfected pools of hippocampal and cortical progenitors in control $\left(W w p 1^{\mathrm{f} / \mathrm{f}} ; W w p 2^{\mathrm{f} / \mathrm{f}}\right)$ and $W w p 1 / 2 \mathrm{dcKO}$ mice in utero at E14.5 with plasmids encoding for GFP and myr-Venus to visualize cellular morphology of the neurons. Brains were fixed at P21, sectioned, and immunostained against GFP and Venus. Using STED microscopy, together with Dr. Katrin Willig (MPIem, Göttingen) we imaged dendritic spines on primary branches of apical dendrites of cortical and hippocampal pyramidal neurons (Fig. 24A). Using maximum projections of Z-stacks acquired through $15 \mu \mathrm{m}$ section thickness, we performed basic morphometrics on dendritic spines in control and $W w p 1 / 2 \mathrm{dcKO}$ neurons. 
Strikingly, loss of $W w p$ ligases lead to a significant increase in density of dendritic spines as compared to control neurons in the cortex (Fig. 24B, and 24D; control $1.42 \pm 0.29$ spines/ $\mu \mathrm{m}$; $W w p 1 / 2 \mathrm{dcKO}, 1.81 \pm 0.38$ spines $/ \mu \mathrm{m})$, and a non-significant increase in hippocampal pyramidal neurons (Fig. $24 \mathrm{C}$, and $24 \mathrm{H}$; control, $1.33 \pm 0.33$ spines $/ \mu \mathrm{m} ; W w p 1 / 2 \mathrm{dcKO}, 1.59$ $\pm 0.21 \mathrm{spines} / \mu \mathrm{m})$. Measurements of spine morphology revealed a statistically significant decrease in the length of filopodia upon $W w p 1 / 2 \mathrm{dcKO}$ in hippocampal neurons (control, $1.07 \pm 0.22 \mu \mathrm{m} ; W w p 1 / 2 \mathrm{dcKO}, 0.79 \pm 0.17 \mu \mathrm{m})($ Fig. 24I), and a specific increase in the diameter of mushroom spine head in cortical $W w p l / 2 \mathrm{dcKO}$ neurons (control, $0.65 \pm 0.05$ $\mu \mathrm{m} ; W w p 1 / 2 \mathrm{dcKO}, 0.73 \pm 0.07 \mu \mathrm{m})($ Fig. $24 \mathrm{~F})$. We observed no significant differences in morphological classification of spines in the $W w p 1 / 2$ dcKO animals (Fig. 24G, and Fig. $24 \mathrm{~K})$.

Taken together, depletion of $W w p 1$ and $W w p 2$ from cortical and hippocampal progenitors alters spine morphology and results in an increase of spine density in cortical nerve cells. As such, Wwp ligases control various aspects of neuronal development, polarity specification, neuronal distribution in the cortex, and dendritic spine morphology. 

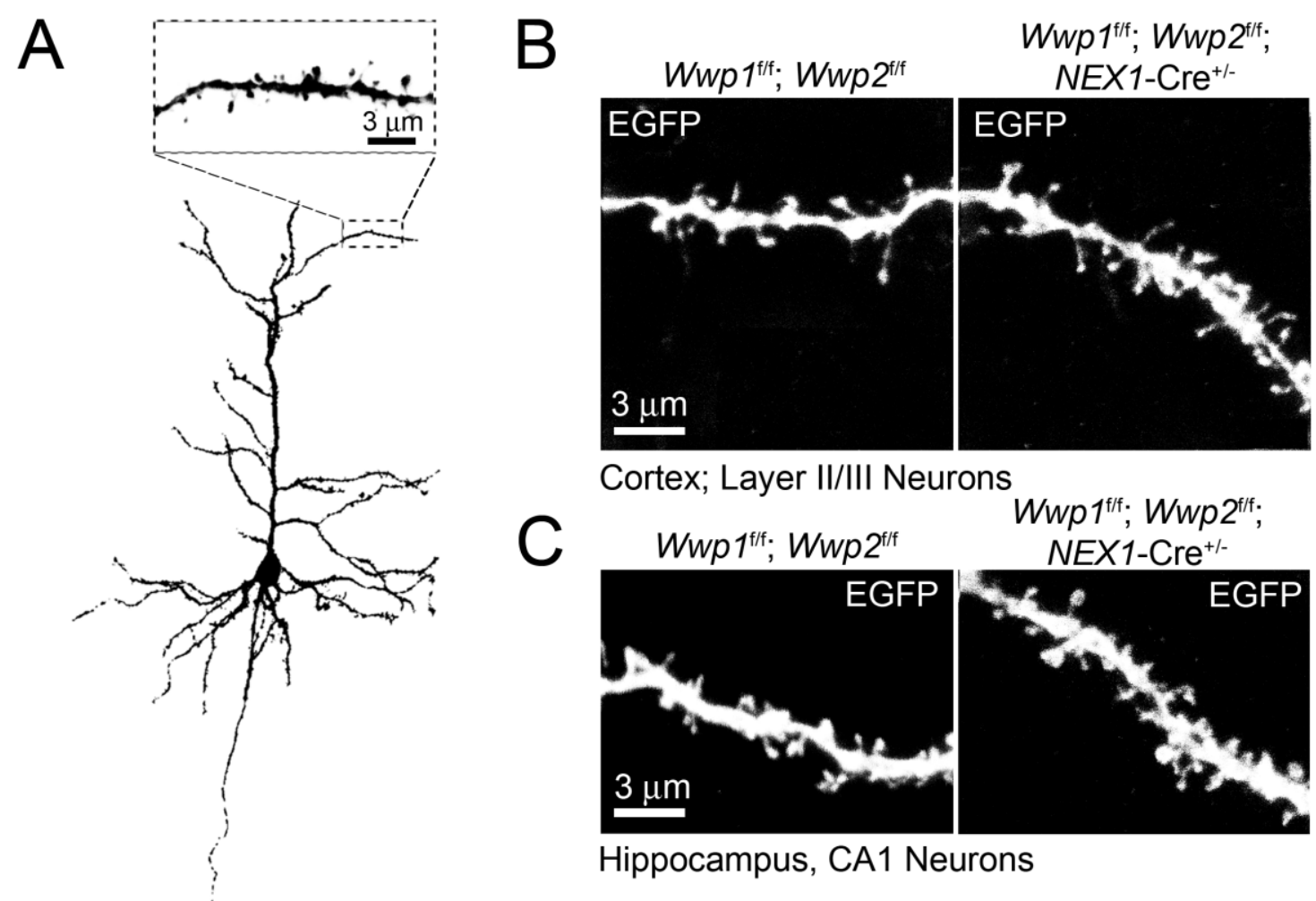

Cortex; Layer II/III Neurons

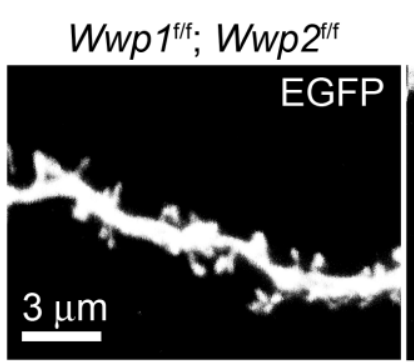

$W w p 1^{f f f} ; W w p 2^{f f f}$

Hippocampus, CA1 Neurons

$D$

$W w p 1^{1 / f ;} ; W w p 2^{f / f}$
$W w p 1^{1 / f /} ; W w p 2^{f / f ;}$

$\mathrm{F}$
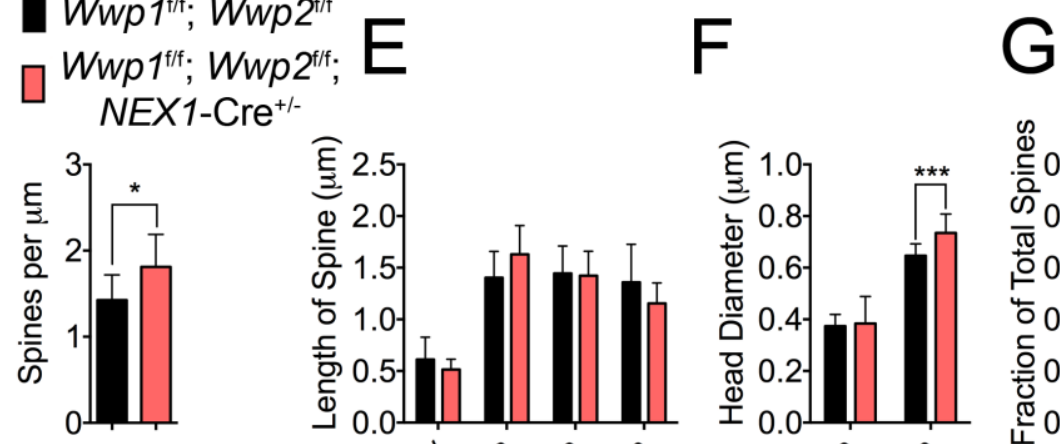

Cortical Layer II/III
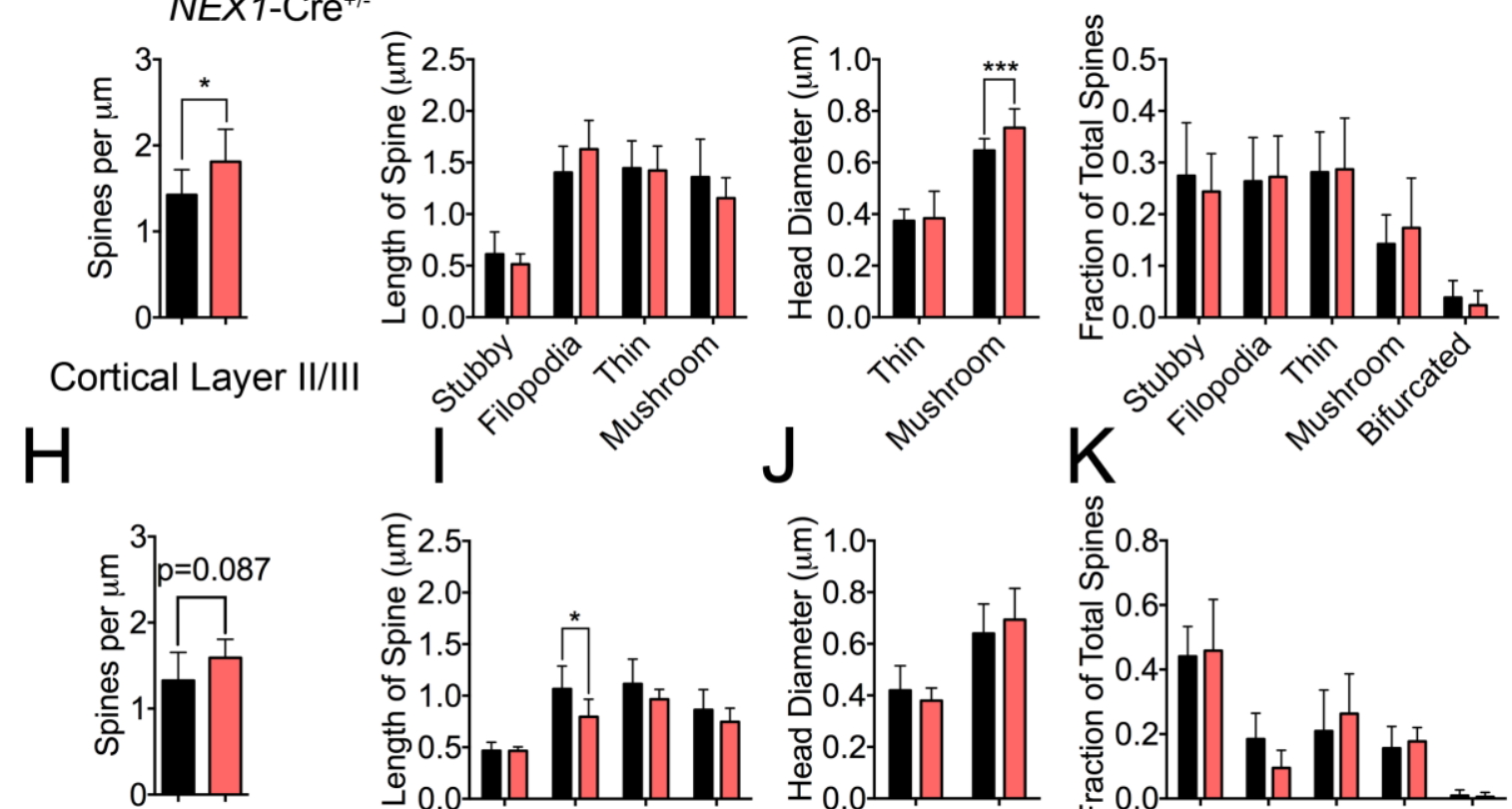

Hippocampus
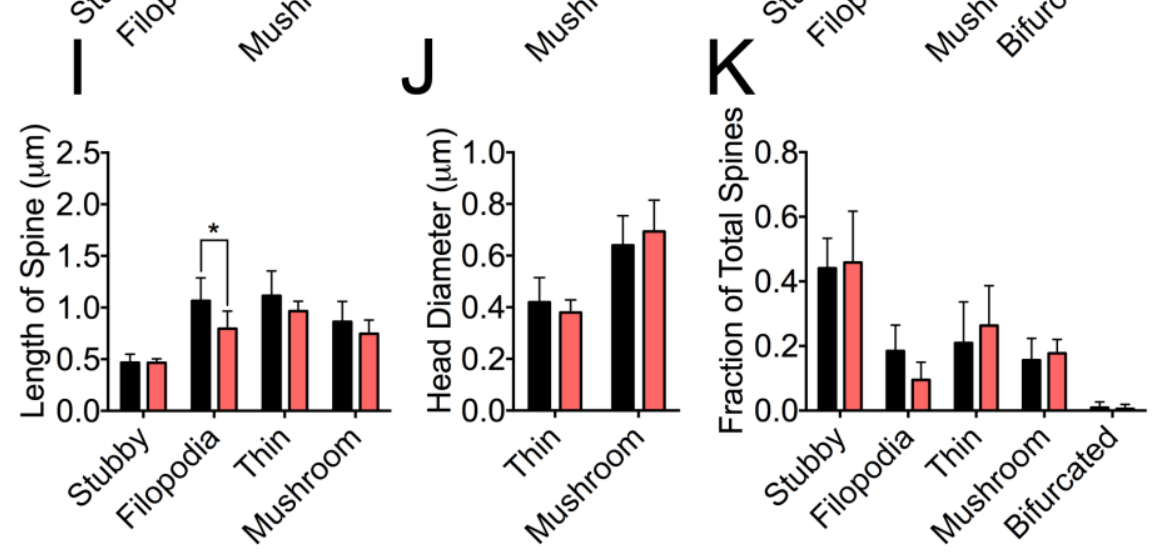

Figure 24. Spine morphometrics in neuron-specific $W w p 1$ and $W w p 2$ double knockout mouse. (A) Tracing of pyramidal cortical neuron of layer II/III expressing GFP and myrVenus. Square in dashed line depicts imaged dendritic compartment of the neuron analyzed. $(\mathrm{B}, \mathrm{C})$ Maximum projections of anti-GFP immunostaining from dendrites of cortical (B) and hippocampal (C) neurons of control (left panel), and $W w p 1$ and $W w p 2$ conditional double 
knockout mice ( $W w p 1 / 2$ dcKO, right panel) acquired with STED microscope. (D to K) Results of morphometrics of spines in cortical (D to G), and hippocampal neurons (H to K). (D, and H) Quantification of spine densities in cortical (D), and hippocampal neurons $(\mathrm{H})$ of control and $W w p 1 / 2$ dcKO mice. (E, and I) Averages of spine lengths in cortical (E), and hippocampal neurons (I) of control and $W w p 1 / 2$ dcKO mice. (F, and J) Averages of spine head diameter in control and $W w p 1 / 2$ dcKO neurons of the cortex $(\mathrm{F})$, and hippocampus $(\mathrm{J})$. (G, and K) Spine classification into morphological groups in cortical (G), and hippocampal neurons $(\mathrm{K})$ of control, and $W w p 1 / 2$ dcKO mice. Bars on all graphs represent averages \pm S.D. Cortex: control, $W w p 1^{\mathrm{f} / \mathrm{f}} ; W w p 2^{\mathrm{f} / \mathrm{f}}, \mathrm{n}=2$ animals, $\mathrm{n}=9$ cells, $\mathrm{n}=186$ spines; $W w p 1 / 2$ $\mathrm{cKO}, W w p 1^{\mathrm{f} / \mathrm{f}} ; W w p 2^{\mathrm{f} / \mathrm{f}} ; N E X 1-\mathrm{Cre}^{+/-}, \mathrm{n}=2$ animals, $\mathrm{n}=8$ cells, $\mathrm{n}=218$ spines; hippocampus: control, $W w p 1^{\mathrm{f} / \mathrm{f}} ; W w p 2^{\mathrm{f} / \mathrm{f}}, \mathrm{n}=2$ animals, $\mathrm{n}=9$ cells, $\mathrm{n}=152$ spines; $W w p 1 / 2$ $c K O, W w p 1^{\mathrm{f} / \mathrm{f}} ; W w p 2^{\mathrm{f} / \mathrm{f}} ; N E X 1-\mathrm{Cre}^{+/-}, \mathrm{n}=1$ animal, $\mathrm{n}=7$ cells, $\mathrm{n}=167$ spines. ${ }^{*} \mathrm{p}<0.05$, $* * * \mathrm{p}<0.001$, unpaired t-test.

\subsubsection{Nedd4-2 regulates spine size and leads to emergence of bifurcated spines}

Nedd4-2 shares similar domain architecture as Wwp1 and Wwp2, and its role in synaptic transmission has been well described before [129]. Our in situ hybridization experiments demonstrate relative abundance of Nedd4-2 mRNA in central murine nervous system (Fig. $25 \mathrm{~A}$ ) and its relative enrichment in the hippocampus (Fig. 25A, enlarged square). In order to study involvement of Nedd4-2 in spinogenesis and its possible control over dendritic spine morphology, we performed in utero transfection of hippocampal progenitors at E14.5 in control $\left(N e d d 4-2^{\mathrm{f} / \mathrm{f}}\right)$ and neuron-specific Nedd4-2 conditional knockout animals (Nedd4-2f/f; NEX1-Cre $\left.{ }^{+/}, \mathrm{Nedd} 4-2 \mathrm{cKO}\right)$ with GFP- and myr-Venus plasmids. We studied the number and morphology of dendritic spines on primary branches of apical dendrites upon Nedd4-2 deletion from hippocampal CA1 pyramidal neurons using STED microscopy (Fig. 25B). Although, dendritic spine density was not affected by Nedd4-2 loss (Fig. 25C), the morphometric analysis of individual spines in Nedd4-2 cKO neurons revealed striking defects. We observed a statistically significant increase in the length of filopodia (control, $0.88 \pm 0.24 \mu \mathrm{m} ;$ Nedd4- $2 \mathrm{cKO}, 1.26 \pm 0.28 \mu \mathrm{m}$ ), thin (control, $0.92 \pm 0.19 \mu \mathrm{m} ;$ Nedd4-2 $\mathrm{cKO}, 1.19 \pm 0.24 \mu \mathrm{m}$ ), and mushroom spines (control, $0.80 \pm 0.16 \mu \mathrm{m} ;$ Nedd4-2 cKO, 1.00 $\pm 0.26 \mu \mathrm{m}$ ) (Fig. 25D), and in the thin spine head size (control $0.35 \pm 0.07 \mu \mathrm{m}$; Nedd4-2 $\mathrm{cKO}, 0.43 \pm 0.09 \mu \mathrm{m}$ ) (Fig. 25E). Additionally, loss of Nedd4-2 leads to a statistically significant decrease in the frequency of mushroom spines (control $0.20 \pm 0.06$; Nedd4-2 $\mathrm{cKO} 0.11 \pm 0.06)$ at the expense of an increased prevalence of the bifurcated type (control $0.03 \pm 0.03 ;$ Nedd4 $-2 \mathrm{cKO} 0.07 \pm 0.04)($ Fig. $25 \mathrm{~F})$. Altogether, loss of Nedd4-2 in neurons 
leads to severe alterations in spine morphology, which places Nedd4-2 among potent regulators of dendritic spine development.

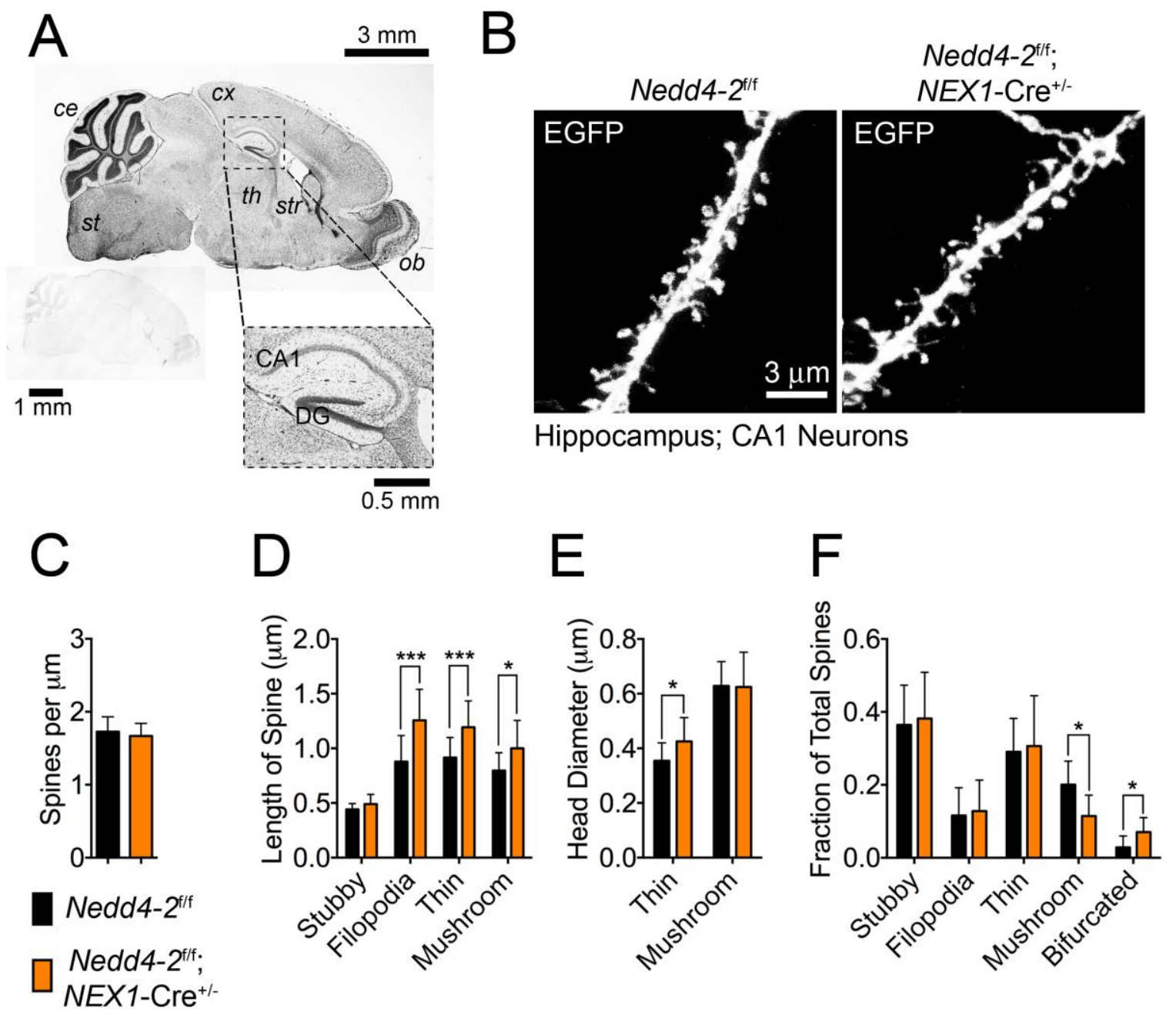

Figure 25. Spine morphometrics in neuron-specific Nedd4-2 conditional knockout mouse. (A) In situ hybridization with probes for Nedd4-2 mRNA on sagittal sections of P20 wild type murine brain. Control reaction with sense probe on the bottom left image. Black signals represent strong hybridization. Brain stem $(s t)$, cerebellum $(c e)$, cortex $(c x)$, thalamus (th), striatum (str), olfactory bulb (ob), Cornu Ammonis area 1 CA1, dentate gyrus DG Nedd4-2 mRNA is enriched in CA1 and dentate gyrus (DG) of hippocampus (enlarged square). (B) Representative maximum projections of STED images of anti-GFP immunolabeling from dendrites of control (Nedd4-2 ${ }^{\mathrm{f} / \mathrm{f}}$, left panel) and neuron-specific Nedd4-2 knockout (Nedd4-2 ${ }^{\text {f/f; }}$ NEX1-Cre ${ }^{+-}$, right panel) CA1 neurons. (C) Quantification of spine densities on Nedd4-2 $2^{\mathrm{f} / \mathrm{f}}$ and Nedd4-2 $2^{\mathrm{f} / \mathrm{f}} ; N E X 1-\mathrm{Cre}^{+/-}$dendrites. (D) Averages of spine lengths in Nedd4-2 $2^{\mathrm{f} / \mathrm{f}}$ and Nedd4-2 ${ }^{\mathrm{f} / \mathrm{f}} ; \mathrm{NEXI}-\mathrm{Cre}^{+/-}$hippocampal neurons. Note consistent increase in spine lengths upon Nedd4-2 deletion (see also B). ${ }^{*} \mathrm{p}<0.05, * * * \mathrm{p}<0.001$, unpaired t-test. (E) Average head diameters in Nedd4-2/f and Nedd4-2 $2^{\mathrm{f} / \mathrm{f}} ; \mathrm{NEX} 1-\mathrm{Cre}^{+/-}$ neurons. Note the thicker heads of thin spines upon Nedd4-2 deletion. $* \mathrm{p}<0.05$, unpaired t-test. (F) Spine classification reveals significant loss of mushroom spines, and gain of bifurcated type in neurons devoid of Nedd4-2. Two way ANOVA with Bonferroni multiple 
comparisons test; $* \mathrm{p}<0.05$. Bars on all graphs represent averages \pm S.D. Control, Nedd4$2^{\mathrm{f} / \mathrm{f}}, \mathrm{n}=2$ animals, $\mathrm{n}=11$ cells, $\mathrm{n}=288$ spines; Nedd4-2 $2^{\mathrm{f} / \mathrm{f}} ; N E X 1-\mathrm{Cre}^{+/-}, N e d d 4-2 \mathrm{cKO}, \mathrm{n}=$ 2 animals, $\mathrm{n}=12$ cells, $\mathrm{n}=315$ spines.

\subsection{HECT type ubiquitin ligase Ube3b is essential for normal neuronal development and function}

\subsubsection{Loss of Ube3b in mice recapitulates human Kaufman oculocerebrofacial syndrome}

Phylogenic tree of HECT-type ubiquitin ligases distinguishes 28 HECT ligases in humans. Over the course of this study, we elucidated that three murine homologs - Wwp1, Wwp2, and Nedd4-2 - regulate dendritic spine number and morphology, implicating their pivotal role in shaping synaptic transmission [92]. To date, four genes encoding human HECT ligases - UBE3A, UBE3B, NEDD4-2, and HUWE1 - have been reported as causative for human disorders $[111,131,136,283,284]$. Among those, $U B E 3 B$ caught our attention. The research groups of Prof. Guntram Borck and Prof. Giuseppe Zampino identified truncating mutations in $U B E 3 B$ in patients suffering from Kaufman oculocerebrofacial syndrome (KOS) [136,138] (Fig. 26A), an autosomal recessive disorder characterized by facial dysmorphisms, developmental delay, and severe mental retardation with absent speech (Fig. $26 \mathrm{~A}, \mathrm{a}, \mathrm{b}, \mathrm{c})$. Some of the neurological aspects of the disease encompass hypoplasia of the corpus callosum, microcephaly (Fig. 26B), and Chiari malformation (Fig. 26C). Cerebellar tonsils are displaced towards the foramen magnum, obtruding the outflow of cerebrospinal fluid, often leading to hydrocephalus in Chiari Type I malformation [285]. UBE3B encodes for ubiquitin ligase E3B. Importantly, truncated UBE3B in KOS patients is devoid of the Cterminal catalytic HECT domain, implicating the loss of enzymatic ubiquitin ligase activity in disease pathology. Although numbers of human mutations in $U B E 3 B$ have been reported in KOS patients [137], no molecular characterization of Ube3b exists to date.

In order to study the role of murine Ube3b (an ortholog of human UBE3B) in the brain, we employed Cre-loxP technology to knockout $U b e 3 b$ in mice (Fig. 26D). Embryonic stem (ES) cell clones with exon 7 of $U b e 3 b$ flanked by two loxP sites and the lacZ/neomycin cassette flanked by two FRT sites (Fig. 26D, depicted as 'Recombined') were purchased from the EUCOMM Consortium. ES cells were expanded and injected to blastocysts of pseudopregnant mice. Chimeric mice with germline transmitted mutant $U b e 3 b$ allele were then 
crossed with FLP deleter mice (B6;SJL ${ }^{\mathrm{Tg}(\mathrm{ACTFLPe})}$, 9205 ${ }^{\text {Dym/J }}$, Jackson Laboratory) to remove lacZ/neo selection cassette from the mutant $U b e 3 b$ allele (Fig. 26D, depicted as 'Floxed'). Then, we crossed mice with floxed Ube3b alleles with Cre-driver lines to delete Ube $3 b$ conventionally and conditionally.

Gene targeting was validated by PCR, Southern, and Western blotting (Fig. 26E to 26G, and Fig. 26J). We performed long range PCR using a primer pair specific to lacZ/neo cassette and to the sequence of $U b e 3 b$ outside of the short arm of the plasmid used for homologous recombination and detected a specific band for the recombined allele in the DNA of heterozygote ES cells (Fig. 26E). Further, we performed multiplex PCR (Fig. 26F) using three primers: a pair recognizing the DNA sequence of wild type Ube3b up-, and downstream of integrated 3' loxP site, and one complementary to loxP. The reaction using mutant DNA yielded two bands, proving correct insertion of 3'loxP sites. Moreover, Southern blotting using a probe outside of the short arm of the targeting vector distinguished correct recombination of the mutant DNA into Ube3b locus (Fig. 26G).

In order to conventionally delete $U b e 3 b$, we crossed $U b e 3 b^{\mathrm{f} / \mathrm{f}}$ mice with E2A-Cre driver mice. Conventional Ube $3 b$ knockout animals $\left(U b e 3 b^{-/}\right)$presented striking resemblance to KOS patients (Fig. 26H). Animals were smaller, severely malnutritioned (probably due to micrognathia or frail teeth), and displayed prominent snout malformations including orbital arch (sometimes preventing the animals from opening the eye), and pronounced kyphosis. $U b e 3 b^{-/-}$mice also presented with a shuffling gait when moving. Examination of $U b e 3 b^{-/-}$ brains revealed microcephaly, corroborating the condition present in KOS patients. Finally, to confirm depletion of Ube $3 b$ in $U b e 3 b^{-/-}$mice, in collaboration with Synaptic Systems (Göttingen) we developed an antibody against HECT domain of Ube3b. Western blotting using brain homogenates from P7 animals showed complete loss of Ube3b in the brains of $U_{b e} b^{-/-}$mice (Fig. 26J). Interestingly, heterozygous animals (middle lane, Fig. 26J) displayed approximately $30 \%$ of Ube $3 b$ level in the brain. 
A

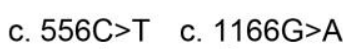

- I10-40-14

$\begin{array}{ccc}\uparrow & \uparrow & \Uparrow \\ \text { c. } 545-2 A>G & \text { c. } 1741+2 \mathrm{G}>\mathrm{C} & \mathrm{C}_{i} 2180 \mathrm{~A}>\mathrm{C}\end{array}$

c.2223_2224delAG
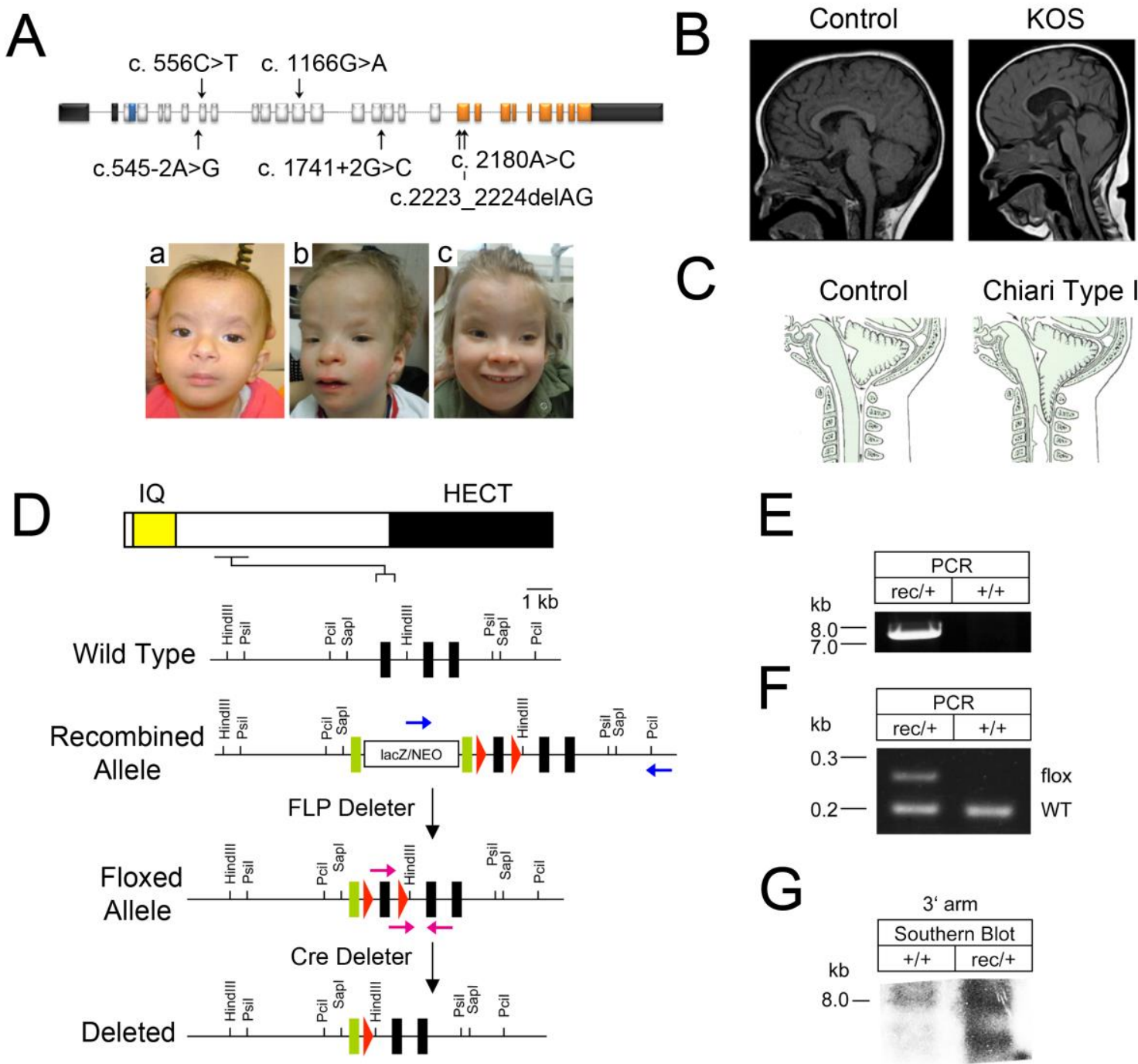

C
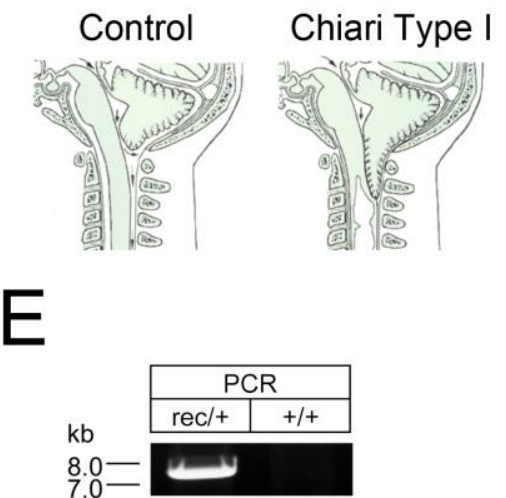

F

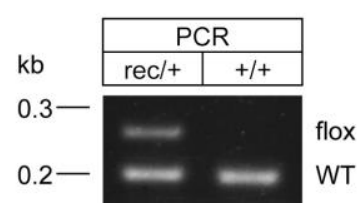

$G$

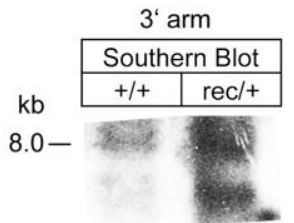

$\mathrm{H}$
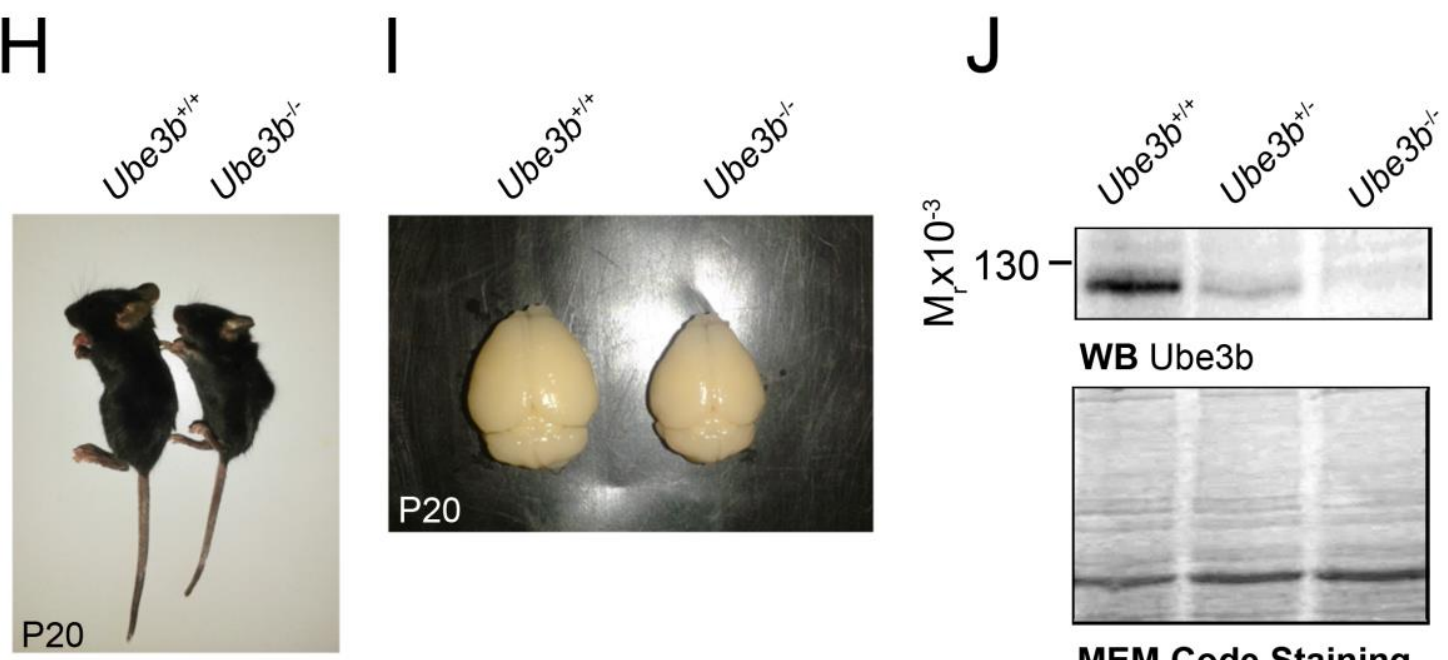

MEM Code Staining

Figure 26. Ube3b knockout in mice recapitulates human Kaufman Oculocerebrofacial Sydrome (KOS). (A) Mutations of $U b e 3 b$ in KOS patients and facial dysmorphisms in KOS. (Top panel) Gene structure of Ube3b. Rectangles, and horizontal lines represent exons and introns. Black, blue, and orange filled rectangles correspond to exons encoding untranslated regions, the IQ motif and the HECT domain, respectively. On top and bottom 
of the scheme, the truncating mutations in patients with KOS are listed. (Bottom panels, a to c) Three individuals with KOS. (B) Cranial MRI scan of a control (left panel) and a KOSdiagnosed individual in photo a in (A) (right panel). (C) Schemes of control brain (left panel) and Chiari Type 1 malformation, present on MRI scan of the KOS individual (right panel). (D) Gene targeting strategy of murine Ube3b. The domain structure of Ube3b, wild type and targeted $U b e 3 b$ alleles are depicted. Exons, loxP sites, FLP-recombinase target sites (FRT) are symbolized as black rectangles, red triangles, and green rectangles, respectively. Exon 7 of $U b e 3 b$ is flanked by two loxP sites. lacZ/neomycin cassette serves as a selection marker. Mutant mice with the recombined allele were crossed with FLP deleter animals to remove the selection cassette, and further crossed to Cre-driver lines, leading to removal of exon 7 conditionally or conventionally. (E, F, G) Validation of Ube $3 b$ gene targeting. +/+ wild type, rec/+ indicates heterozygous recombined mutant. (E) The result of long range PCR using primers depicted as blue arrows in D. Band of the predicted size was observed only using genomic DNA purified from rec/+ mutant as a template. (F) The result of multiplex PCR using primers depicted as pink arrows in D. PCR using genomic DNA from targeted ES cells yields two bands (left lane), and PCR using wild type ES cells genomic DNA as a template results in one band. (G) Southern blotting analysis of genomic DNA purified from targeted ES cells. Genomic DNA isolated from control and ES cells carrying 'Recombined' allele of $U b e 3 b$ was digested with SapI enzyme. The band at $8.0 \mathrm{~kb}$ represents the wild type allele, and the band at $6.6 \mathrm{~kb}$ represents the mutant allele. (H) Gross morphology of $U b e 3 b^{+/+}$(left) and $U b e 3 b^{-/-}$(right) mice at 20 days post birth. (I) Brains of $U b e 3 b^{+/+}$(left) and Ube $3 b^{-/-}$ (right) mice isolated 20 days after birth. (J) Results of Western blotting using P7 brain lysates from $U b e 3 b^{+/+}$(left), $U b e 3 b^{+/-}$(middle), and $U b e 3 b^{-/}$(right) animals with an antibody raised against HECT domain of Ube $3 \mathrm{~b}$. MEM Code staining (lower panel) displays comparable amounts of total protein in all lanes after SDS-PAGE, and electrotransfer onto the nitrocellulose membrane. (A) and (B) adapted from [136,138], and published under Creative $\begin{array}{llll}\text { Commons } & \text { license. } & \text { (C) } & \text { from }\end{array}$ http://neuros.net/de/images/chiari_malformation_de.gif.

\subsubsection{Ube3b is expressed in developing and adult murine central nervous system and is enriched at PSDs}

As a basic characterization of Ube $3 \mathrm{~b}$, we studied the expression of this ligase in murine brain (Fig. 27). We designed a DIG-probe recognizing mRNA sequence spanning the middle region and a portion of HECT domain of Ube3b (top scheme, Fig. 27A), and performed in situ hybridization using sagittal brain sections from a mouse embryo (top panel, Fig. 27A) and an adult (bottom panel, Fig. 27A). Notably, we detected strong hybridization signal in the ventricular zone of the developing cortex and ganglionic eminences. Consistently in the adult brain, $U b e 3 b$ transcript was abundant and expressed throughout entire central nervous system, with strong hybridization signals from the cerebral cortex and hippocampus, indicative of a putative role of Ube $3 \mathrm{~b}$ in learning, memory, and higher cognitive functions, (Fig. 27A). Next, we performed developmental expression profiling of Ube3b by Western 
blotting using cortical lysates from mice at prenatal to postnatal stages (Fig. 27B). Interestingly, Ube3b was expressed at constant levels throughout embryonic development and its expression increased 6-fold from P0 to P21 to reach a plateau phase (Fig. 27C). We next analyzed the distribution of Ube $3 \mathrm{~b}$ in different regions of P56 mouse brain. The level of Ube $3 b$ was higher in the cerebral cortex, olfactory bulb, and hippocampus as compared to other regions of the central nervous system (i.e. cerebellum, midbrain, striatum, pons, thalamus, and medulla) (Fig. 27D).

In the next experiment, we examined subcellular localization of Ube $3 b$ in brain cells. For this purpose, we purified subcellular fractions from $6-7$ weeks old animals (Fig. 27E, also Fig. 19A). Strikingly, Ube3b was predominantly found in the PSD fraction (Fig. 27F; compare the top panel for PSD-95 with the bottom panel for Ube3b), indicating that this ligase associates with postsynaptic specialization in neurons.

Taken together, Ube $3 b$ is expressed in developing, and mature cortical pallium where it seems to be an important regulator of the postsynapse. 

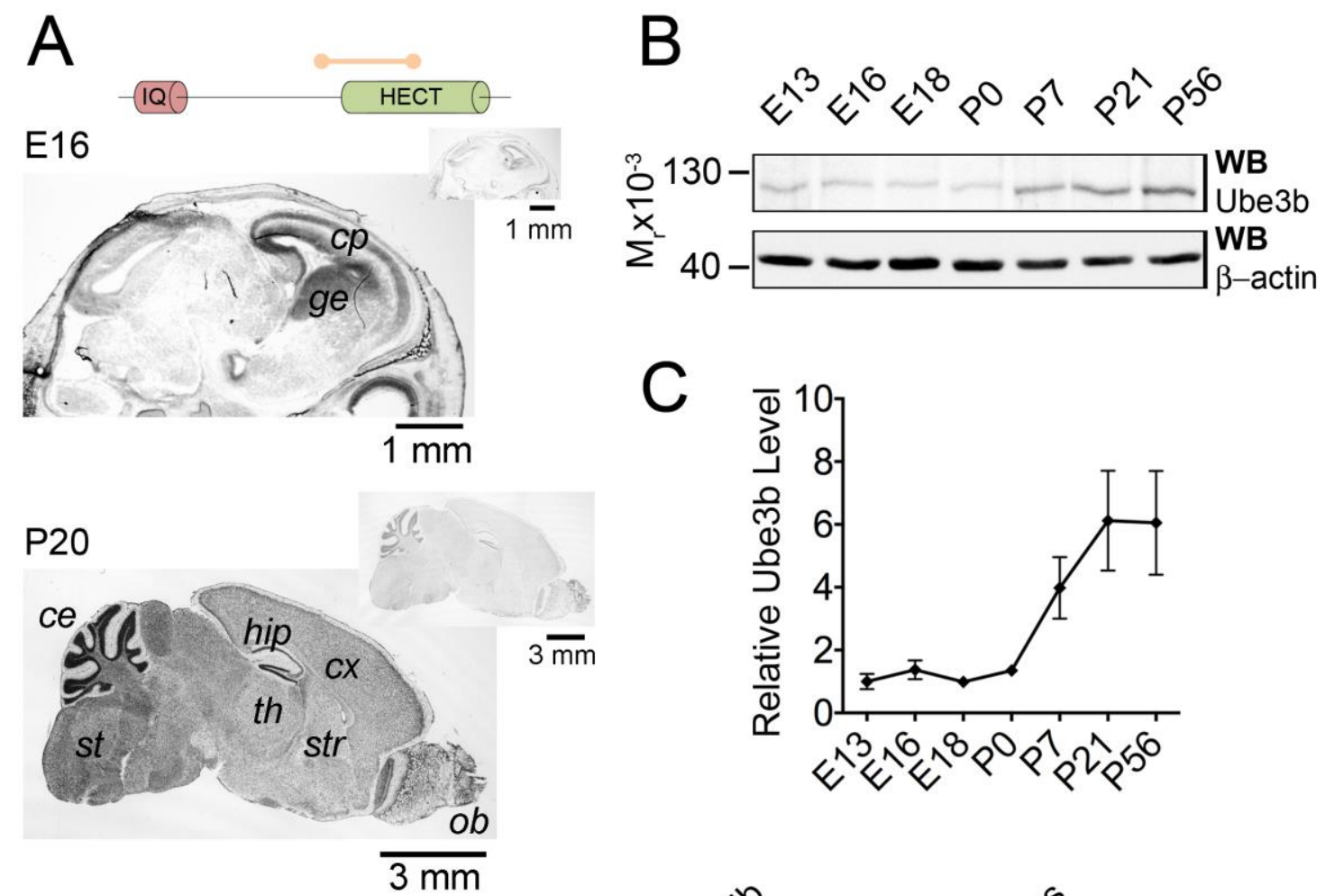

$D$

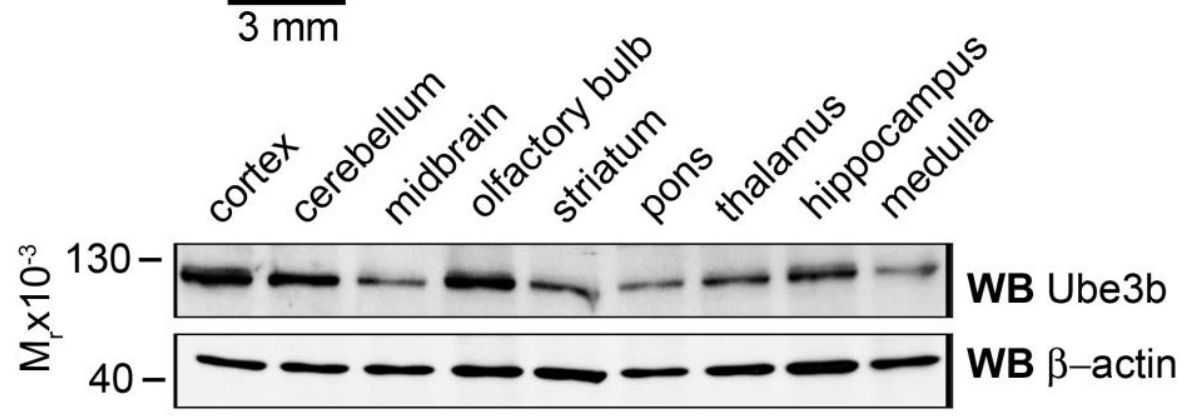

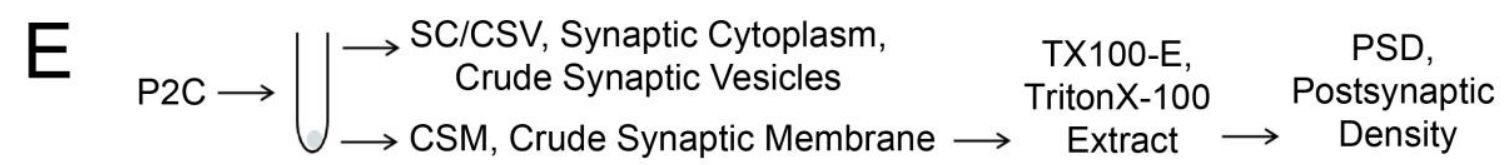

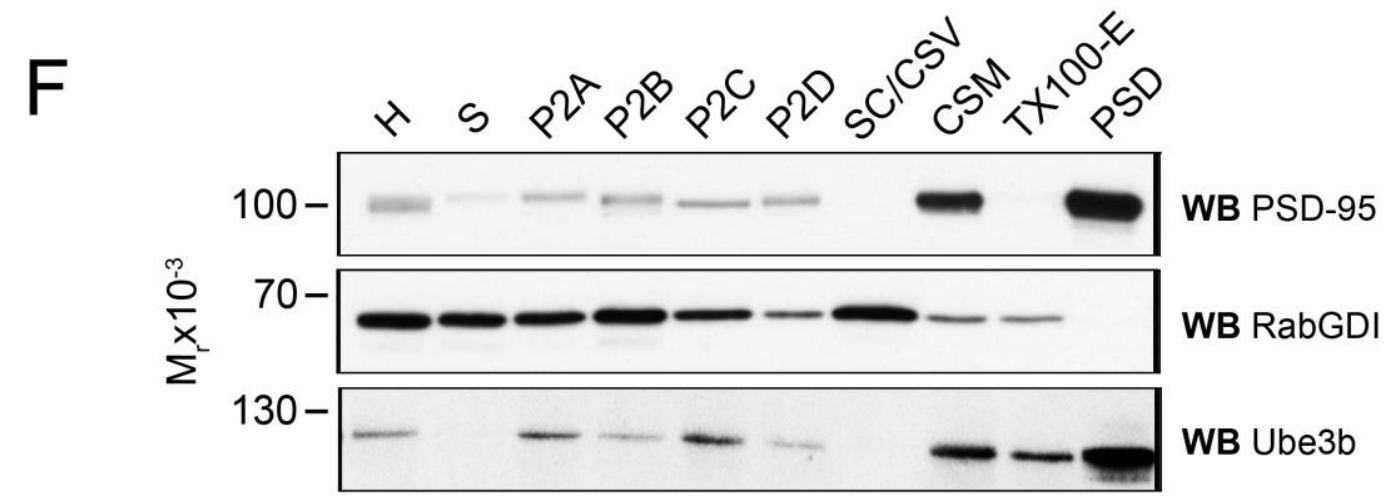

Figure 27. Ube3b is expressed in developing and mature murine central nervous system. (A) In situ hybridization on sagittal sections of E16 (upper panel) and P20 (lower panel) of mouse brains with antisense probe for mRNA encoding HECT domain of Ube $3 \mathrm{~b}$ (top scheme) and sense control probe (right top panels). Note the enrichment of Ube $3 b$ mRNA in developing cortex (cortical plate, $c p$ ), and ganglionic eminence ( $g e$ ), and overall 
abundance in the adult central nervous system (cerebellum, ce; brain stem, st; hippocampus, hip; cortex, $c x$; thalamus, $t h$; striatum, str; olfactory bulb $o b$ ). (B) Developmental expression profile of Ube $3 \mathrm{~b}$. Cortical homogenates at different developmental stages (indicated on top panel) were used for Western blotting with antibodies against Ube $3 \mathrm{~b}$ (top panel), and $\beta$-actin (bottom panel, loading control). (C) Quantification of Ube3b levels in mouse cortex during brain development. Ube $3 b$ level was normalized to $\beta$-actin and expressed relative to the ligase level in E13 embryonic cortex. Each point represents mean Ube3b level from three cortices \pm S.E.M. (D) Western blotting using homogenates of different adult mouse brain regions with antibodies against Ube $3 b$ (top panel), and $\beta$-actin (bottom panel, loading control). (E) Scheme of brain fractionation in sucrose gradient after synaptosomes purification (see also Fig. 19A). After osmotic shock, synaptosomal fraction were further centrifuged to separate synaptic cytoplasm (SC) / crude synaptic vesicles (CSV) from crude synaptic membrane (CSM). CSM is treated with TritonX-100-containing buffer to separate the detergent insoluble postsynaptic density (PSD) from TritonX-100-extractable fraction (TX100-E). (F) Results of Western blotting using subcellular fractions (compare also Fig. 19A, and Fig. 27E). Note that Ube3b signal, like PSD-95 signal, is highly enriched in PSD fraction.

\subsubsection{Ube3b knockout abrogates neurite branching in primary hippocampal neurons}

Impaired dendritogenesis has been long known as a major aspect of a plethora of intellectual disability syndromes [286-289]. In order to study the impact of Ube3b loss on dendrite development, we analyzed the morphology of primary hippocampal neurons prepared from $U b e 3 b^{\mathrm{f} / \mathrm{f}}$ mice expressing GFP (control), or GFP and Cre recombinase (Ube3b KO). Already at DIV4, Ube3b KO neurons display pronounced deficits in neurite branching (Fig. 28A). Next, we infected $U b e 3 b^{\mathrm{f} / \mathrm{f}}$ primary hippocampal neurons with lentiviruses expressing GFP, or Cre recombinase and concluded that at DIV5, $U b e 3 b^{\mathrm{f} / \mathrm{f}}$ neurons expressing Cre recombinase are almost completely devoid of Ube3b. Then, we measured the length of axons, identified as the longest neurite projected by a single neuron. Notably, Ube $3 b \mathrm{KO}$ neurons projected significantly less primary branches at DIV4 as compared to control (Fig. 28C; control, $4.06 \pm 2.03$ primary branches; Ube $3 b \mathrm{KO}, 2.71 \pm 1.88$ primary branches). At a later stage of neuronal development, DIV7 (Fig. 28D), we quantified the complexity of neurites by Sholl analysis (Fig. 28E, and 28F). For this purpose, concentric circles at $7.5 \mu \mathrm{m}$ intervals were drawn on the binarized image of the GFP signal from an individual neuron and numbers of neurites crossing each circle were counted [290]. Ube3b KO neurons display less complex neurite branching as compared to control nerve cells (Fig. 28E), with fewer 
overall numbers of crossings (control, $61.7 \pm 20.7$ crossings; Ube $3 b \mathrm{KO}, 93.1 \pm 31.4$ crossings) (Fig. 28F).

To study if impaired dendritogenesis is a specific consequence of Ube $3 b$ enzymatic activity loss, we transfected $U b e 3 b^{\mathrm{f} / \mathrm{f}}$ primary hippocampal neurons with plasmids encoding GFP alone (control), or GFP and Cre (Ube3b KO) together with either myc-tagged wild type Ube3b (Fig. 28I, and 28J), or with the catalytically inactive point mutant of Ube $3 b$ (Ube3b C/S) (Fig. 28G, and 28H). Experiments described in Figs. 28G to 28L were performed as a single set of experiments with the ones in Figs. 28D to 28F. Upon expression of Ube $3 \mathrm{~b} \mathrm{C/S}$ mutant in the $U b e 3 b \mathrm{KO}$ neurons, the complexity of neurites was similar to the one of $U b e 3 b$ KO neurons expressing only GFP (compare Fig. 28D, right panel, and 28G). On the contrary, expression of wild type Ube $3 \mathrm{~b}$ in Ube $3 b \mathrm{KO}$ neurons restores neurite branching (compare Fig. 28D, left panel, and Fig. 28I). The impact of re-expression of wild type Ube3b in Ube $3 b$ $\mathrm{KO}$ neurons on the neurite branching was significantly stronger than that of expression of Ube $3 \mathrm{~b} \mathrm{C} / \mathrm{S}$ (Figs. $28 \mathrm{~K}$, and 28L; the sum of total crossings of neurites, Ube $3 b \mathrm{KO}+\mathrm{Ube} 3 \mathrm{~b}$, $81.1 \pm 24.1 ;$ Ube $3 b \mathrm{KO}+\mathrm{Ube} 3 \mathrm{~b} \mathrm{C} / \mathrm{S}, 57.6 \pm 20.4)$. Interestingly, the expression of wild type Ube $3 b$ in the control neurons had almost no effect on neurite arborizations (compare Fig. 28I, and 28J). Possibly, endogenous Ube3b expression level required for proper dendritic branching is saturated.

Expression of Ube $3 \mathrm{~b} \mathrm{C} / \mathrm{S}$ in control neurons resulted in statistically significant decrease in the sum of crossing neurites as compared to control neurons expressing GFP (Figs. 28F, and 28L; control neurons+Ube3b C/S, $61.8 \pm 21.6$; control neurons+GFP, $93.1 \pm 31.4$; also compare Fig. 28D, and Fig. 28H). We conclude that this indicates dominant-negative effects of Ube $3 \mathrm{~b} \mathrm{C/S}$ overexpression, as experiments in Figs. $28 \mathrm{D}$ to $28 \mathrm{~F}$ were performed simultaneously with those presented on Figs. $28 \mathrm{G}$ to $28 \mathrm{~L}$. Additionally, overexpression of Ube $3 \mathrm{~b} \mathrm{C/S}$ has almost no effect in the Ube3b KO background (Figs. 28K, and 28L, also compare Figs. 28G, and 28H). 

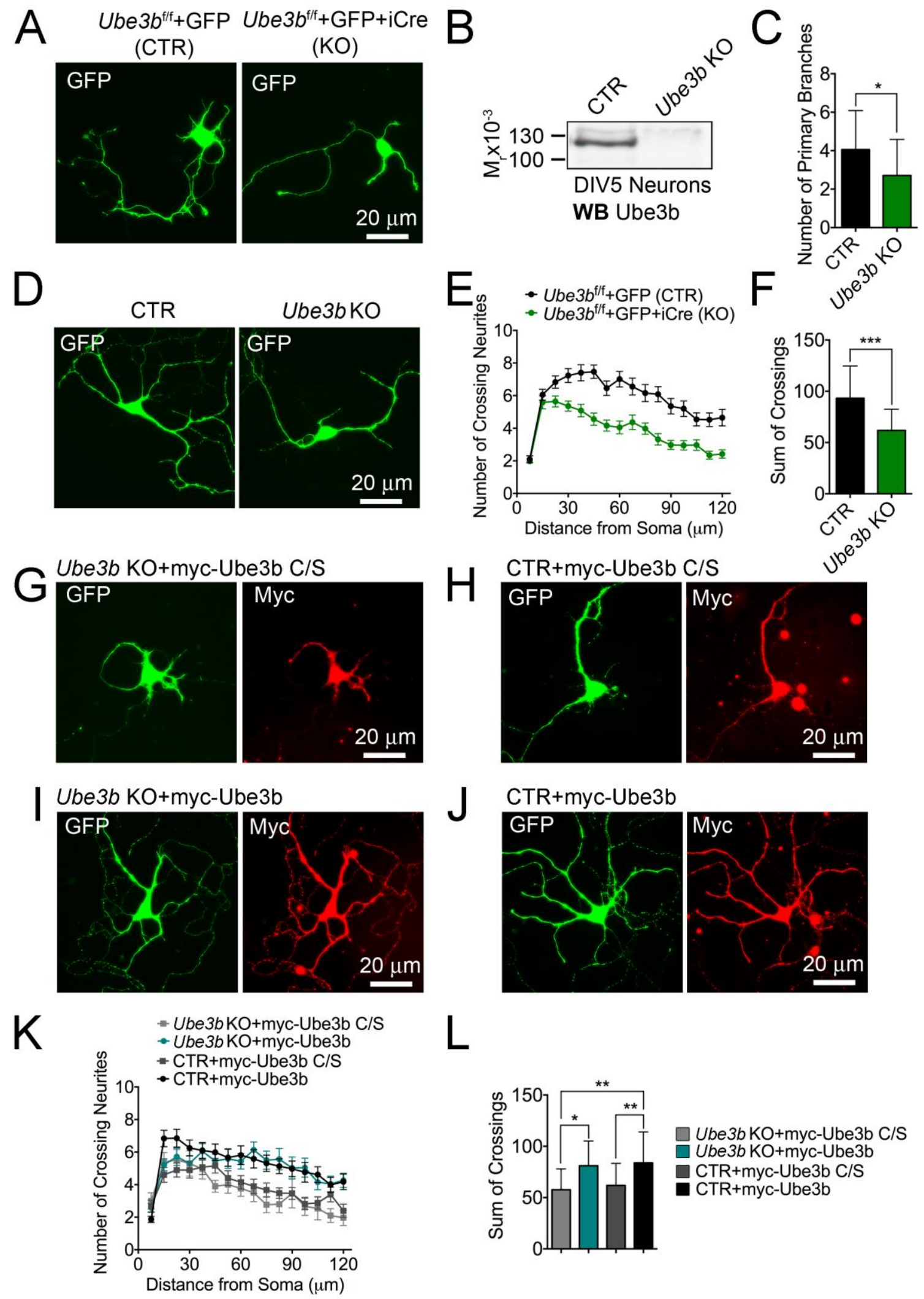

Figure 28. Ube3b loss in neurons leads to dramatic impairment of neurite branching. (A) Images of primary hippocampal neurons at day 4 in vitro (DIV4) prepared from Ube $3 b^{\mathrm{f} / \mathrm{f}}$ mice and transfected with calcium phosphate method either with plasmid encoding GFP (control, CTR), or plasmid co-expressing GFP and Cre recombinase to induce Ube $3 b$ knockout (KO). (B) Western blotting against Ube3b in neuronal lysates. Primary 
hippocampal neurons from $U b e 3 b^{\mathrm{f} / \mathrm{f}}$ mice were infected at DIV1 with lentiviruses expressing GFP (CTR), or Cre recombinase (KO), and harvested at DIV5. (C) Mean numbers of primary axonal branches, counted in DIV4 control and KO neurons. The longest neurite projected by an individual neuron was measured as an axon. CTR, $\mathrm{n}=36 ; \mathrm{KO}, \mathrm{n}=24 ;{ }^{*} \mathrm{p}=0.012$, unpaired t-test. (D) Images of control (CTR) and KO primary hippocampal neurons expressing GFP at DIV7. (E) Sholl analyses of control and KO neurons. Each point represents mean number of crossing neurites with concentric circles emerging from the soma \pm S.E.M. CTR, $\mathrm{n}=47$; KO, $\mathrm{n}=42$ neurons. (F) Average sum of total crossings with Sholl circles from a single neuron. CTR, $\mathrm{n}=47 ; \mathrm{KO}, \mathrm{n}=42$ neurons; $* * * \mathrm{p}<0.001$, unpaired $\mathrm{t}$ test. (G) Example images of GFP fluorescence (left panel) and myc immunostaining (right panel) from KO neuron transfected with plasmids encoding for GFP and myc-tagged catalytically inactive mutant of Ube3b (myc-Ube3b C/S). (H) Example images of a CTR neuron expressing GFP (left panel) and myc-Ube $3 \mathrm{~b} \mathrm{C} / \mathrm{S}$ (right panel). (I) GFP fluorescence (left) and myc-staining (right panels) from $\mathrm{KO}$ neuron expressing myc-tagged wild type Ube3b (myc-Ube3b). (J) A control neuron expressing GFP (left panel) and myc-Ube3b. (K) Sholl analyses of neurite branching in CTR and KO neurons expressing myc-Ube $3 b$, or mycUbe $3 \mathrm{~b} \mathrm{C/S}$. (L) Mean sum of crossing neurites with Sholl circles made by single neurons. $\mathrm{KO}+$ myc-Ube $3 \mathrm{~b} \mathrm{C} / \mathrm{S}, \mathrm{n}=15 ; \mathrm{KO}+$ myc-Ube3b, $\mathrm{n}=23 ; \mathrm{CTR}+$ myc-Ube $3 \mathrm{~b} \mathrm{C} / \mathrm{S}, \mathrm{n}=24$; $\mathrm{CTR}+$ myc-Ube $3 \mathrm{~b}, \mathrm{n}=34$ neurons. One-way ANOVA of the interaction between the genotype and myc-Ube $3 b$ variants, $* * * p=0.0007$. Tukey's multiple comparisons between $\mathrm{KO}+$ myc-Ube $3 \mathrm{~b}$ and $\mathrm{KO}+$ myc-Ube $3 \mathrm{~b} \mathrm{C} / \mathrm{S}, * \mathrm{p}<0.05$; between $\mathrm{CTR}+$ myc-Ube $3 \mathrm{~b}$ and $\mathrm{CTR}+$ myc-Ube3b C/S, ** $\mathrm{p}<0.01$; between $\mathrm{CTR}+$ myc-Ube $3 \mathrm{~b}$ and $\mathrm{KO}+$ myc-Ube3b C/S, $* * \mathrm{p}<0.01$. Bar graphs represent averages \pm S.D. Scale bar, $20 \mu \mathrm{m}$.

\subsubsection{Pathogenic point mutations in UBE3B abrogate neurite branching}

During the course of this study, our collaborating group of Guntram Borck, identified additional two point substitution mutations in HECT domain, Arg997Pro (R997P) and Gly779Arg (G779R) present in patients with KOS [137]. Those patients display intellectual disabilities with absent speech, and patients bearing G779R are diagnosed with seizures. Additionally, KOS patients display a number of non-neuronal symptoms (e.g. hypocholesterolemia or elevated thyroid-stimulating hormone, TSH in serum [136]. Our $U_{b e} 3 b^{-/-}$mice showed a general developmental delay, resembling KOS patients. In order to circumvent secondary effects caused by non-neuronal developmental defects, to study the role of Ube3b in the brain, we generated neuron- and glia-specific conditional Ube $3 b \mathrm{KO}$ $\left(U b e 3 b^{\mathrm{f} / \mathrm{f}} ; E m x 1-\mathrm{Cre}^{+/}\right.$, Ube $\left.3 b \mathrm{cKO}\right)$ mouse line (Fig. 29A). In Emxl-Cre ${ }^{+/-}$driver line, Cre is expressed in Emxl-positive cell lineage, which gives rise to glutamatergic neurons and glia of the forebrain $[203,291]$. Next, we confirmed almost complete loss of Ube $3 b$ from the cortex of Ube3b cKO mice by Western blotting (Fig. 29B). Residual Ube3b expression 
comes likely from non-Emxl expressing cells, i.e. GABAergic inhibitory neurons. Additionally, this indicates that Ube $3 b$ is expressed in the neurons and glia of the forebrain. In the next set of experiments, we studied the consequences of G779R and R997P mutations for the role of Ube3b in neurite branching. We prepared primary hippocampal neurons from mice and expressed GFP as a control (Fig. 29C), or co-expressed GFP and HA-tagged wild type human UBE3B (HA-hUBE3B; Fig. 29D), GFP and HA-tagged G779R mutant (HAhUBE3B G779R; Fig. 29E), or GFP and HA-tagged R997P mutant of hUBE3B (HAhUBE3B R997P; Fig. 29F) by calcium phosphate transfection. All UBE3B plasmids were generated and provided by Rüstem Yilmaz and Guntram Borck (University of Ulm). Neurons were fixed and immunolabeled with anti-HA at DIV7. Overexpression of human $\mathrm{UBE} 3 \mathrm{~B}$ in $U b e 3 b \mathrm{cKO}$ neurons results in an increase of neurite branching as compared to GFP expressing Ube3b cKO neurons (Figs. 29C, 29D, 29G, 29H). This indicates conserved role of $U b e 3 b$ in neuronal development. Strikingly, expression of both point mutants of UBE3B fails to rescue defective neurite branching phenotype (compare Figs. 29C with 29E, and 29F). Sholl analyses and comparison of mean sums of crossing neurites showed significant differences between $U b e 3 b \mathrm{cKO}$ neurons expressing HA-hUBE3B, and HAhUBE3B G779R, and between Ube3b cKO neurons expressing HA-hUBE3B, and HAhUBE3B R997P (Figs. 29G, and 29H; Ube3b cKO, $24.3 \pm 10.5$ crossings; Ube3b cKO+hUBE3B, $35.5 \pm 15.9$ crossings; Ube3b cKO+hUBE3B G779R, $22.8 \pm 9.3$ crossings; Ube3b cKO+hUBE3B R997P $24.1 \pm 8.6$ crossings). Of these mutants, HA-hUBE3B R997P displayed a peculiar perinuclear localization, while N-terminally tagged HA-hUBE3B was abundantly expressed in neurons at DIV7 (Fig. 29F, and Fig. 29C), In conclusion, G779 and R997 of UBE3B mutated in patients with KOS are essential for its function in neurite development and R997 is crucial for proper subcellular localization of UBE3B in the nerve cell. 

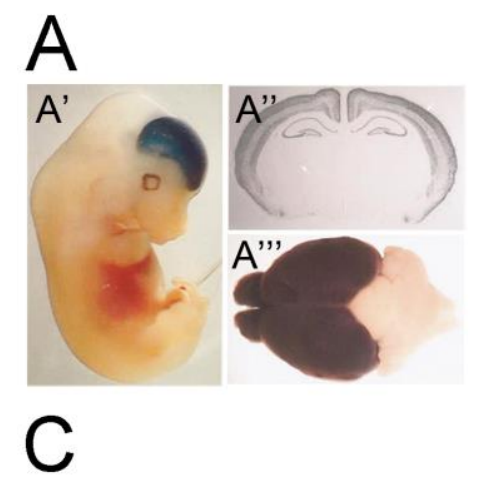

Ube $3 b^{\text {fff; }} ; \mathrm{Em} \times 1-\mathrm{Cre}^{+/-}(\mathrm{cKO})+\mathrm{GFP}$
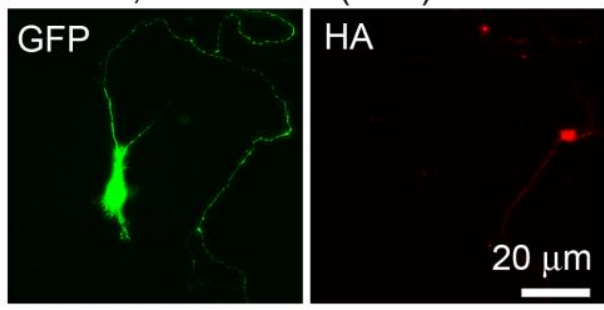

$\mathrm{E}$

Ube3b cKO+GFP+HA-hUBE3B G779R
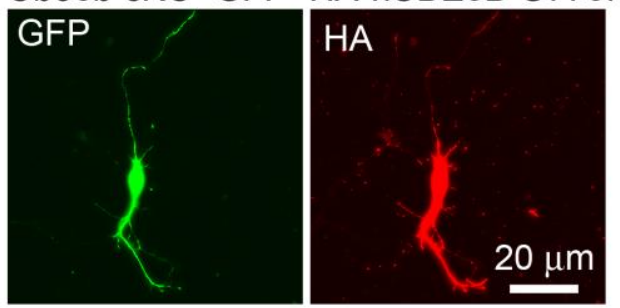

G

- Ube $3 b$ cKO+GFP

* Ube3b cKO+GFP+HA-hUBE3B

- Ube3b cKO+GFP+HA-hUBE3B G779R

- Ube3b cKO+GFP+HA-hUBE3B R997P

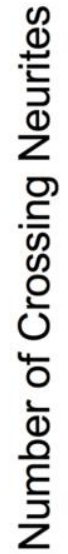

10
8
6
4
0
0

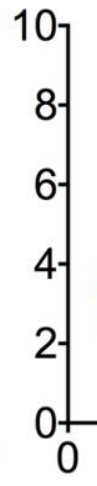

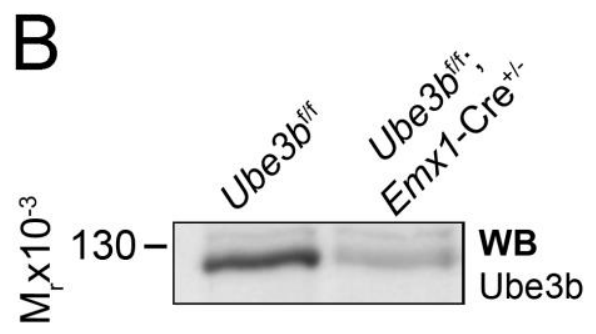

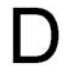

Ube $3 b$ cKO+GFP+HA-hUBE3B
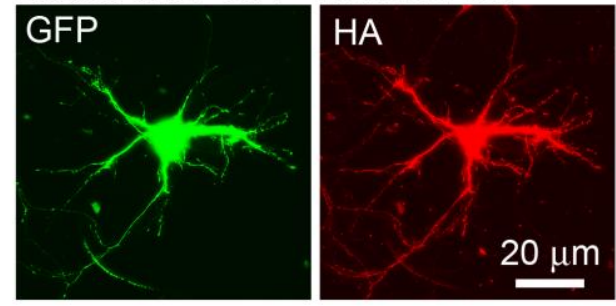

$\mathrm{F}$

Ube3b cKO+GFP+HA-hUBE3B R997P

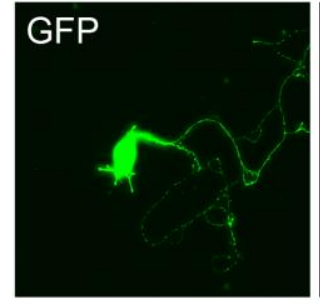

$\mathrm{HA}$

$\mathrm{H}$

$20 \mu \mathrm{m}$

Figure 29. Pathogenic point mutations in $U B E 3 B$ identified in KOS patients abrogate neurite branching. (A) Reporter $\beta$-galactosidase stainings of knock-in Emxl-Cre ${ }^{+/-}$mice. In this mouse line, lacZ reporter and Cre gene is inserted directly into exon 1 of the EmxI gene. Histochemical staining of the reporter to track brain regions subjected to Cre-mediated recombination in E12.5 embryo (A'), the coronal brain section (A'), and the entire brain of 
an adult mouse (A"'), (B) Validation of Ube3b loss in the conditional, Emxl-Cre ${ }^{+/}$driven Ube3b knockout (Ube3b cKO) cortex. Western blotting using cortical lysates of adult control (Ube $3 b^{\mathrm{f} / \mathrm{f}}$, left lane) and Ube $3 b \mathrm{cKO}\left(U b e 3 b^{\mathrm{f} / \mathrm{f}} ; E m x 1-\mathrm{Cre}^{+/-}\right.$, right lane) mice with the antibody against Ube3b. (C to F) Primary hippocampal neurons from Ube $3 b^{\mathrm{f} / \mathrm{f}} ; E m x 1$ $\mathrm{Cre}^{+/-}$(Ube3b cKO) mice transfected with plasmids encoding GFP and variants of HAtagged human UBE3B. Neurons were fixed at DIV7. Representative images of GFP (left panels) and HA staining (right panels) in Ube $3 b$ cKO neuron expressing GFP alone (C), GFP with HA-tagged wild type human UBE3B (HA-hUBE3B) (D), GFP with HA-tagged human UBE3B G779R point mutant (HA-hUBE3B G779R) (E), GFP with HA-tagged human UBE3B R997P point mutant (HA-hUBE3B R997P) (F). Scale bar, $20 \mu \mathrm{m}$. (G) Sholl analyses of $U b e 3 b \mathrm{cKO}$ neurons expressing indicated proteins. Each point represents mean number of crossing neurites with concentric circles emerging from the soma \pm S.E.M. (F) Averaged sum of total crossings with Sholl circles until $50 \mu \mathrm{m}$ from the center of the soma. $U b e 3 b \mathrm{cKO}+\mathrm{GFP}, \mathrm{n}=33$; Ube $3 b \mathrm{cKO}+\mathrm{GFP}+\mathrm{HA}-\mathrm{hUBE} 3 \mathrm{~B}, \mathrm{n}=15 ;$;be $3 b \mathrm{cKO}+\mathrm{GFP}+\mathrm{HA}-$ hUBE3B G779R, $\mathrm{n}=31$; Ube3b cKO+GFP+HA-hUBE3B R997P, $\mathrm{n}=24$. One-way ANOVA of the interaction between the genotype and HA-hUbe $3 b$ variants, $* * p=0.002$. Tukey's multiple comparisons between Ube $3 b \mathrm{cKO}+\mathrm{GFP}$ and Ube $3 b \mathrm{cKO}+\mathrm{GFP}+\mathrm{HA}-$ hUBE3B, ** $\mathrm{p}<0.01$; between Ube3b cKO+GFP+HA-hUBE3B and Ube $3 b$ $\mathrm{cKO}+\mathrm{GFP}+\mathrm{HA}-\mathrm{hUBE} 3 \mathrm{~B}$ G779R, ** $\mathrm{p}<0.01$, and between Ube3b cKO+GFP+HAhUBE3B and Ube3b cKO+GFP+HA-hUBE3B R997P, ** $\mathrm{p}<0.01$. Bar graphs represent averages \pm S.D.

(A) After [291]. Adapted in line with Open Access Publishing convention.

\subsubsection{Neuron and glia specific Ube3b knockout mice display reduction of cortical thickness}

Next, we examined the gross morphology of the brain in $U b e 3 b \mathrm{cKO}$ mice. Brains from 9 weeks old $U b e 3 b \mathrm{cKO}\left(\mathrm{Ube}^{\mathrm{f}} \mathrm{b}^{\mathrm{f} / \mathrm{f}} ; E m x \mathrm{I}-\mathrm{Cre}^{+/-}\right)$male mice reveal a non-significant tendency towards a reduction in cortical thickness as compared to control (Ube $\left.3 b^{\mathrm{f} / \mathrm{f}}\right)$ mice (control, $1.00 \pm 0.16$; Ube3b cKO, $0.85 \pm 0.12 ; \mathrm{p}=0.051$; Fig. 30A, and 30B), while numbers of cortical neurons in the coronal cross sections show no difference between control and Ube $3 b$ cKO (control, $1.00 \pm 0.22$; Ube3b cKO, $0.99 \pm 0.19$; Fig 30C). Consistently, there was a slight trend towards an increased density of neurons in the Ube $3 b \mathrm{cKO}$ brains as compared to control (control, $1.00 \pm 0.34 ;$ Ube $3 b \mathrm{cKO}, 1.14 \pm 0.25 ;$ Fig. 30D), indicating that reduced neurite arborization upon Ube $3 \mathrm{~b}$ loss might be the primary cause of reduction of cortical thickness in Ube3b cKO mice. Bielschowsky silver impregnation revealed no apparent differences in defects of myelinated axonal tracts in Ube $3 b$ cKO brains. Nonetheless, we noticed prominent ventricular dilatation upon Ube3b cKO (Fig. 30E), corroborating the condition described for KOS patients. 
Hydrocephalus has been described as a cause of reactive-astrogliosis, accumulation of macrophages in the brain and neuronal death by several independent research groups [292294]. In order to exclude the possibility that neuronal loss is the cause of thinner cortex in Ube $3 b \mathrm{cKO}$, we examined control and $U b e 3 b \mathrm{cKO}$ brains for astrogliosis by immunostaining of coronal brain sections for astrocytic marker, glial fibrillary acidic protein (GFAP). As shown in Fig. 30F, we found no obvious difference in staining intensity between control and $U b e 3 b \mathrm{cKO}$.

Next, we assessed inflammatory response as a readout of ongoing apoptosis or degeneration in control and $U b e 3 b \mathrm{cKO}$ brains. We immunostained coronal sections of control and $U b e 3 b$ cKO brains with antibodies for markers for activated microglia (Ionized calcium-binding adapter molecule 1, Iba1, Fig. 30G) or macrophages (Lysosomal-associated membrane protein 2, Lamp-2, also known as Mac3, Fig. 30H), and detected no obvious differences in staining intensities.

Altogether, conditional deletion of $U b e 3 b$ from neurons and glia leads to a tendency towards thinner cerebral cortex, probably due to impairment in neurite branching. 


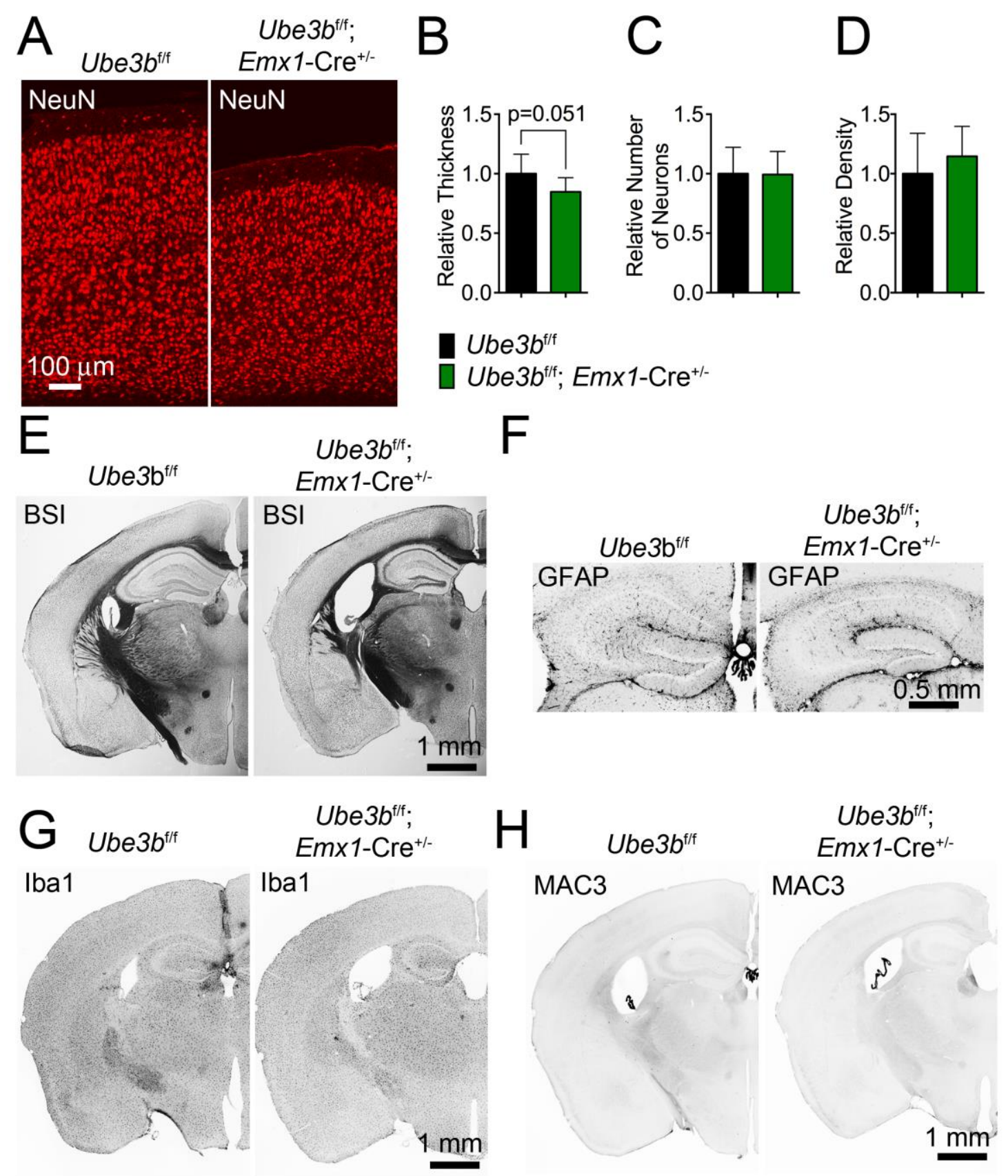

Figure 30. Morphological defects in brain-specific Ube3b conditional knockout mice. (A) Reduced cortex thickness in $U b e 3 b^{\mathrm{f} / \mathrm{f}} ; E m x l-\mathrm{Cre}^{+/-}$mice. Representative images of immunostaining with antibodies for neuronal nuclear marker, NeuN. Coronal sections through somatosensory cortex of $U b e 3 b^{\mathrm{f} / \mathrm{f}}$ (left panel) and $U b e 3 b^{\mathrm{f} / \mathrm{f}} ; E m x 1-\mathrm{Cre}^{+/-}$mouse (right panel) were immunolabeled. Scale bar, $100 \mu \mathrm{m}$. (B) Mean cortical thickness from pial surface to the bottom edge of cortical layer VI in $U b e 3 b^{\mathrm{f} / \mathrm{f}}$ and $U b e 3 b^{\mathrm{f} / \mathrm{f}} ; \mathrm{Emxl} \mathrm{l}-\mathrm{Cre}^{+/-}$mice. Values are expressed relative to thickness of control cortex. (C) Averaged numbers of neurons in $U b e 3 b^{\mathrm{f} / \mathrm{f}}$ and $U b e 3 b^{\mathrm{f} / \mathrm{f}} ; E m x l-\mathrm{Cre}^{+/-}$cortices. The number of NeuN-positive nuclei in a cortical region of $560 \mu \mathrm{m}$ width was counted, and expressed relative to the value in $U b e 3 b^{\mathrm{f} / \mathrm{f}}$ cortex. (D) Averaged neuronal densities in $U b e 3 b^{\mathrm{f} / \mathrm{f}}$ and $U b e 3 b^{\mathrm{f} / \mathrm{f}} ; E m x 1-\mathrm{Cre}^{+/-}$ cortices quantified as a number of NeuN-positive nuclei over the cortex area and expressed relative to $U b e 3 b^{\mathrm{f} / \mathrm{f}}$. Bar diagrams in $\mathrm{B}, \mathrm{C}$, and D represent average \pm S.D. (E) Bielschowsky 
silver impregnation (BSI), and immunostainings using antibodies against GFAP (F), Ibal $(\mathrm{G})$, and MAC3 $(\mathrm{H})$ on coronal brain sections of $U b e 3 b^{\mathrm{f} / \mathrm{f}}$, and $U b e 3 b^{\mathrm{f} / \mathrm{f}} ; E m x I-\mathrm{Cre}^{+/-}$mice. Samples sizes in (B, C, and D) Ube $3 b^{\mathrm{f} / \mathrm{f}}, \mathrm{n}=3$; Ube $3 b^{\mathrm{f} / \mathrm{f}} ; \operatorname{Emxl}_{1}-\mathrm{Cre}^{+/-}, \mathrm{n}=3$; (B) $\mathrm{p}=0.051$, unpaired t-test.

\subsubsection{Increased spine density and longer spines with enlarged heads upon Ube3b deletion in hippocampal neurons}

One of the common features characterized for intellectual disability syndromes, such as Angelman syndrome, Rett syndrome, Down's syndrome, X-linked intellectual disability is altered spine number and/or spine morphology [286,295-297]. Therefore, we studied the features of dendritic spines in CA1 hippocampal neurons in control and Ube $3 b \mathrm{cKO}$ mice. We transfected hippocampal progenitors in utero at E14.5 with GFP and myr-Venus encoding plasmids to visualize dendritic spines. Brains were fixed at P21, when synaptogenesis in mouse brain has been accomplished and dendritic branches stabilized [46]. Upon imaging of GFP and myr-Venus signals from primary branches of apical dendrites projected by CA1 neurons with confocal microscopy, we noted a dramatic increase in spine density in Ube3b cKO neurons as compared to control (Fig. 31A). Moreover, spine morphology seemed markedly altered, hinting at longer spines with wider heads. In order to perform spine morphometrics with super-resolution microscopy, together with Dr. Katrin Willig (MPIem, Göttingen), we employed STED microscopy (Fig. 31B). As illustrated on Figs. 31D, and 31E, we observed a marked statistically significant increase in spine density in Ube $3 b$ cKO neurons as compared to control (control, $1.45 \pm 0.32$; Ube $3 b \mathrm{cKO}, 1.82 \pm$ 0.31 spines $/ \mu \mathrm{m}$ dendrite) (Fig. 31D). In addition, spine heads of thin spines were larger in Ube $3 b \mathrm{cKO}$ neurons (head diameter; control, $0.36 \pm 0.08 \mu \mathrm{m} ; U b e 3 b \mathrm{cKO}, 0.44 \pm 0.05 \mu \mathrm{m}$ ). We observed a slight trend towards increased mushroom spine head diameter upon $U b e 3 b$ cKO, however, not statistically significant. To verify, if observed alterations in dendritic spine number and morphology were mediated by Ube3b in cell-autonomous manner, we in utero electroporated E14.5 hippocampal progenitors of $U b e 3 b^{\mathrm{f} / \mathrm{f}}$ mice with plasmids encoding GFP and myr-Venus (control), or GFP, myr-Venus, and Cre recombinase (Ube3b KO) and analyzed dendritic spines in P21 CA1 pyramidal neurons (Fig. 31C). Expression of Cre was verified by immunostaining with anti-Cre antibody (data not shown). Cre expressing neurons displayed similar, even more pronounced changes in terms of spine number and morphology as compared to nerve cells with Ube3b deleted by Emx1-Cre 
expression (compare Fig. 31D, and Fig. 31F, also Fig. 31B, and Fig. 31C, right panels). Cremediated deletion of $U b e 3 b$ led to an increase in spine density (control, $1.51 \pm 0.09 ; U b e 3 b$ $\mathrm{KO}, 2.20 \pm 0.30$ spines $/ \mu \mathrm{m}$ dendrite) (Fig. 31F). Moreover, statistically significant increase in diameters of heads in $U b e 3 b \mathrm{KO}$ was congruent with observed alterations in $U b e 3 b \mathrm{cKO}$ neurons and affected both classes of head-endowed spines: thin, (control, $0.38 \pm 0.06 \mu \mathrm{m}$; Ube3b KO, $0.48 \pm 0.06 \mu \mathrm{m}$ ) and mushroom type (control, $0.64 \pm 0.13 \mu \mathrm{m}$; Ube $3 b \mathrm{KO}, 0.78$ $\pm 0.11 \mu \mathrm{m})$ (Fig. 31G). Further, we observed a statistically significant increase in the length of spines upon Cre-mediated Ube3b loss in each morphological class: stubby (control, 0.43 $\pm 0.05 \mu \mathrm{m} ; U b e 3 b \mathrm{KO}, 0.50 \pm 0.06 \mu \mathrm{m}$ ), filopodia (control, $0.92 \pm 0.28 \mu \mathrm{m} ; U b e 3 b \mathrm{KO}, 1.15$ $\pm 0.20 \mu \mathrm{m}$ ), thin (control $0.95 \pm 0.18 \mu \mathrm{m} ;$ Ube3b KO, $0.11 \pm 0.13 \mu \mathrm{m}$ ), and mushroom (control $0.82 \pm 0.23 \mu \mathrm{m} ;$ Ube3b KO, $1.09 \pm 0.19 \mu \mathrm{m}$ ) (Fig. 31H). No differences concerning the frequency of spine types between control and $U b e 3 b \mathrm{KO}$ neurons were observed (Fig. 31I). Altogether, Ube3b emerges as a pivotal ubiquitin ligase controlling spine number and morphology in hippocampal neurons. 

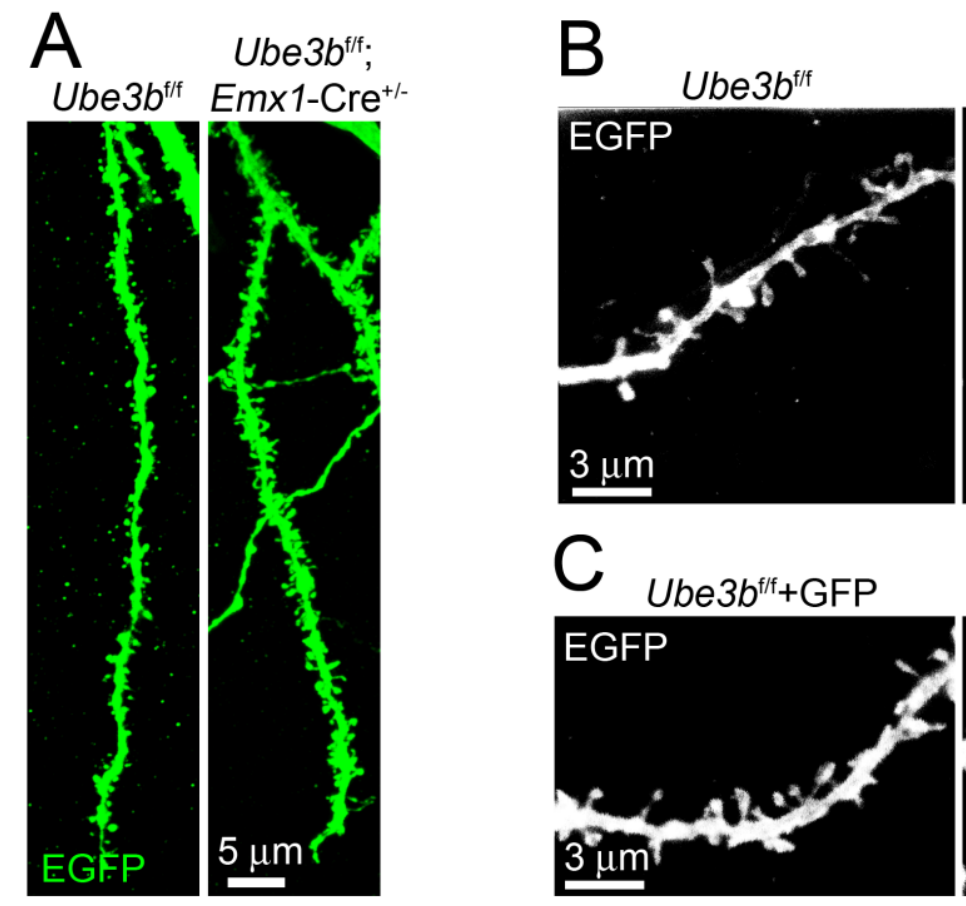

Ube $3 b^{f / 4}$

Emx1-Cre ${ }^{+/-}$
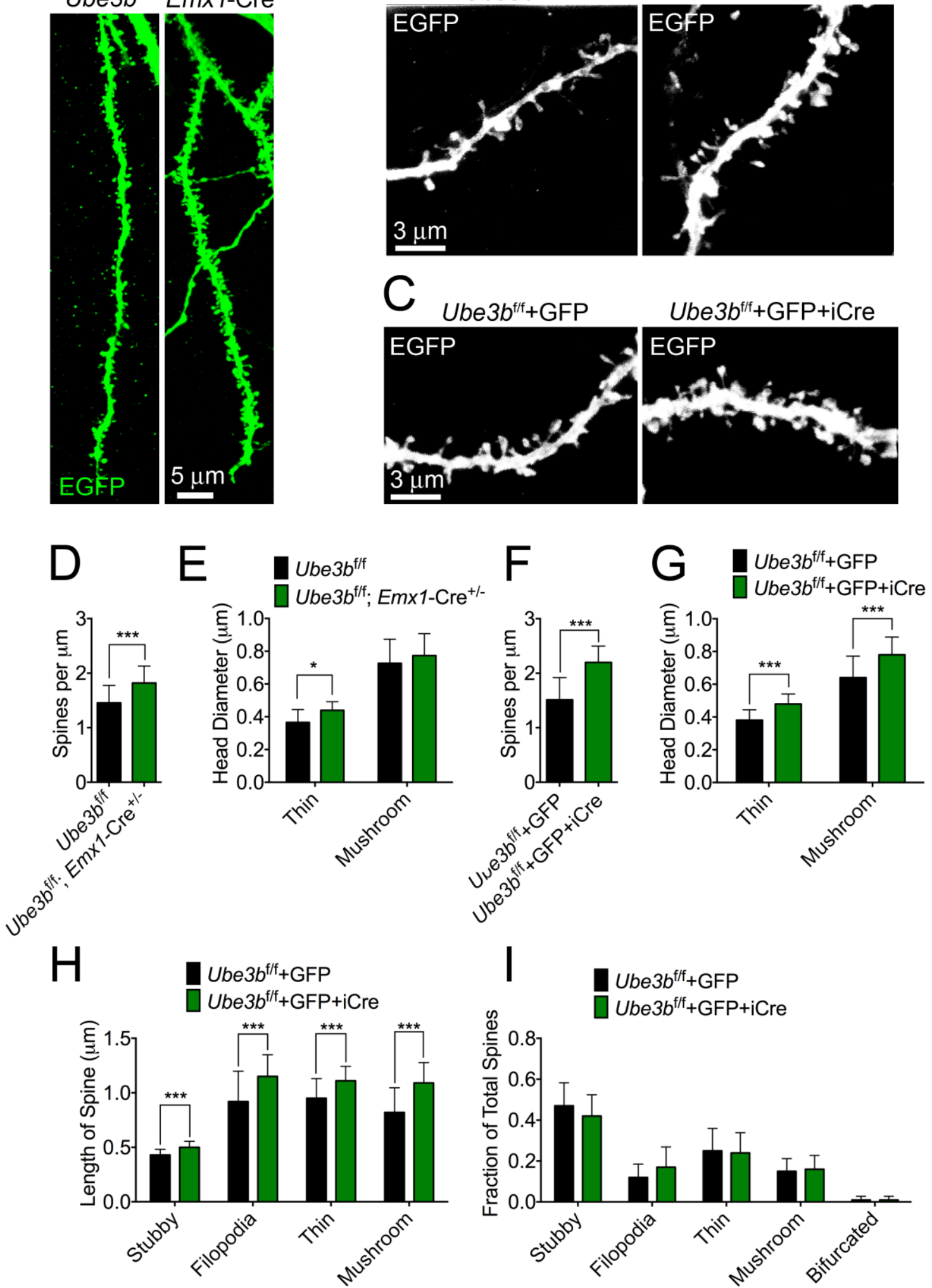

Figure 31. Ube3b deletion in neurons induces morphological aberrances in dendritic spines. (A) Representative images of anti-GFP immunostaining signal from primary apical branches of CA1 pyramidal neurons in $U b e 3 b^{\mathrm{f} / \mathrm{f}}$ (control, left panel) and Ube $3 b^{\mathrm{f} / \mathrm{f}} ;$ Emx 1 $\mathrm{Cre}^{+/-}$(Ube3b cKO, right panel) mice. Hippocampal progenitors were in utero electroporated at E14.5 with plasmids encoding for GFP and myr-Venus and brains were fixed, sectioned, 
and stained at P21. (B) Maximum intensity projections of STED images of anti-GFP immunostaining signal from dendrites of CA1 pyramidal neurons of control (left panel) and $U b e 3 b \mathrm{cKO}$ (right panel) mice. (C) Maximum intensity projections of STED images of antiGFP immunostaining signal from dendrites of $U b e 3 b^{\mathrm{f} / \mathrm{f}}$ hippocampal neurons transfected in utero at E14.5 with plasmids encoding GFP and myr-Venus (control, left panel) or GFP, myr-Venus, and Cre recombinase (Ube3b KO, right panel). (D) Quantification of spine densities. Counted were number of spines per $\mu \mathrm{m}$ dendrite in control and $U b e 3 b \mathrm{cKO}$ CA1 hippocampal neurons (compare also with B). ${ }^{*} \mathrm{p}=0.012$, unpaired t-test. (E) Averaged head diameters of thin, and mushroom spines in control and Ube $3 b$ cKO CA1 hippocampal neurons. $* \mathrm{p}=0.024$, unpaired t-test. (F) Averaged spine densities in GFP (control), or GFP and Cre (Ube $3 b \mathrm{KO}$ ) expressing Ube $3 b^{\mathrm{f} / \mathrm{f}}$ hippocampal neurons. $* * * \mathrm{p}<0.001$, unpaired $\mathrm{t}-$ test. (G) Averaged head diameters of thin and mushroom spines in control and Ube $3 b \mathrm{KO}$ neurons. $* * * \mathrm{p}<0.001$, unpaired t-test. $(\mathrm{H})$ Averaged spine length, measured from the emergence of spine on the dendrite until the head tip. $* * * \mathrm{p}<0.001$, unpaired t-test. (I) Dendritic spines in control and Ube $3 b \mathrm{KO}$ neurons were classified into morphological groups. The graph represents the frequencies of spines of each class. Two-way ANOVA with Sidak's multiple comparisons revealed no significant interaction between the genotype and classes of spines. Data on all bar graphs are represented as averages \pm S.D. Control, $U b e 3 b^{\mathrm{f} / \mathrm{f}}, \mathrm{n}=1$ animal, $\mathrm{n}=10$ cells, $\mathrm{n}=205$ spines; Ube $3 b \mathrm{cKO}, U b e 3 b^{\mathrm{f} / \mathrm{f}} ; E m x 1-\mathrm{Cre}^{+/-}, \mathrm{n}=$ 2 animals, $\mathrm{n}=11$ cells, $\mathrm{n}=286$ spines. Control, $U b e 3 b^{\mathrm{f} / \mathrm{f}}+\mathrm{GFP}, \mathrm{n}=6$ animals, $\mathrm{n}=21$ cells, $\mathrm{n}=457$ spines; Ube $3 b \mathrm{KO}, U b e 3 b^{\mathrm{f} / \mathrm{f}}+\mathrm{GFP}+\mathrm{iCre}, \mathrm{n}=7$ animals, $\mathrm{n}=19$ cells, $\mathrm{n}=649$ spines.

\subsubsection{Increased synaptic transmission in Ube3b KO neurons}

Morphological aberrances involving branching deficiencies and spine morphology alterations upon $U b e 3 b$ deletion from neurons prompted us to study the effects of Ube $3 b$ loss on synaptic transmission. Together with Dr. Silvia Ripamonti and Dr. Jeong Seop Rhee, we analyzed glutamatergic autaptic hippocampal neurons from $U b e 3 b^{\mathrm{f} / \mathrm{f}}$ (control) and $U b e 3 b^{\mathrm{f} / \mathrm{f}} ; E m x 1-\mathrm{Cre}^{+/-}$mice (Ube3b cKO) with electrophysiological methods [298].

We first recorded evoked excitatory postsynaptic currents (EPSCs) by depolarizing the cell from -70 to $0 \mathrm{mV}$ at a frequency of $0.2 \mathrm{~Hz}$ (Fig. 32A). We observed a slight tendency of an increase in mean amplitude for Ube $3 b \mathrm{cKO}$ neurons as compared to control $(\mathrm{p}=0.176)$. Additionally, we detected no differences in capacitance, reflecting no differences in soma sizes between control and Ube3b cKO neurons (Fig. 32J). Next, we analyzed the basic parameters of presynaptic release in control and $U b e 3 b \mathrm{cKO}$ neurons, such as readily releasable pool (RRP) size, vesicular probability of release (Pvr), and short-term plasticity (STP). The size of RRP, measured as the charge transferred during application of hypertonic sucrose solution (Figs. 32B, and Fig. 32D), and Pvr measured as integral of response transferred during an action potential evoked EPSC divided by the charge transferred during sucrose application (Fig. 32E) were not affected upon $U b e 3 b \mathrm{cKO}$ in nerve cells. During the 
application of stimuli at $10 \mathrm{~Hz}$, the EPSC amplitudes recorded from control and $U b e 3 b \mathrm{cKO}$ neurons declined equally, indicating that STP is not altered upon deletion of Ube $3 b$ (Fig. $32 \mathrm{~F}$ ). These findings indicate that presynaptic release in nerve cells is not affected by the loss of Ube $3 b$.

Increased spine densities in mature neurons observed upon Ube3b loss (Fig. 31) can lead to an increase in the number of synapses formed by mutant nerve cell. We then analyzed miniature EPSCs reflecting spontaneous, action potential-independent release of neurotransmitter at the synapse (Fig. 32G). While the amplitudes of mEPSCs were not altered (Fig. 32H), the frequency of mEPSCs was dramatically increased (Fig. 32I) in Ube3b cKO neurons compared to control nerve cells (control, $5.33 \pm 0.82 \mathrm{~Hz}$; Ube $3 b$ cKO, $9.45 \pm$ $1.39 \mathrm{~Hz}$ ). These findings illustrate that upon $U b e 3 b$ loss, the number of functional synapses is increased, while the number of functional receptors on a single dendritic spine is unaltered in $U b e 3 b$ cKO neurons.

In order to examine the number of postsynaptic receptors expressed on the cell surface, we recorded postsynaptic currents induced by exogenous application of $100 \mu \mathrm{M}$ glutamate (Fig. $32 \mathrm{~K}$ ) or $3 \mu \mathrm{M} \gamma$-aminobutyric acid (GABA) (Fig. 32L) in control and mutant neurons. While we detected no differences in the response to exogenous GABA application (Fig. 32O), there was a trend towards an increase in current amplitude following exogenous glutamate application in $U b e 3 b \mathrm{cKO}$ neurons (control, $1.02 \pm 0.10 \mathrm{nA} ; U b e 3 b \mathrm{cKO}, 1.31 \pm 0.12 \mathrm{nA} ; \mathrm{p}$ $=0.063$; Fig. $32 \mathrm{~N}$ ). In order to further examine glutamatergic transmission in Ube $3 b \mathrm{cKO}$ neurons, we analyzed the abundance of AMPA- and NMDA-glutamate receptors by measuring the ratio between the currents passing through both receptor types. Interestingly, we observed increased NMDA/AMPA ratio for $U b e 3 b \mathrm{cKO}$ neurons as compared to control (control, $0.18 \pm 0.02$; Ube $3 b \mathrm{cKO}, 0.29 \pm 0.04 ; \mathrm{p}=0.013$; Fig. 32P), indicating alterations of composition of postsynaptic glutamate receptors in mutant nerve cells. 
A

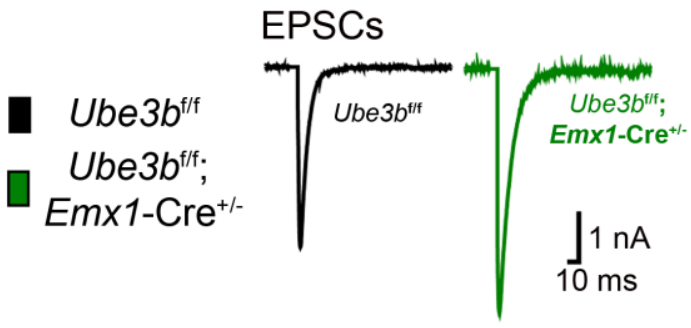

B
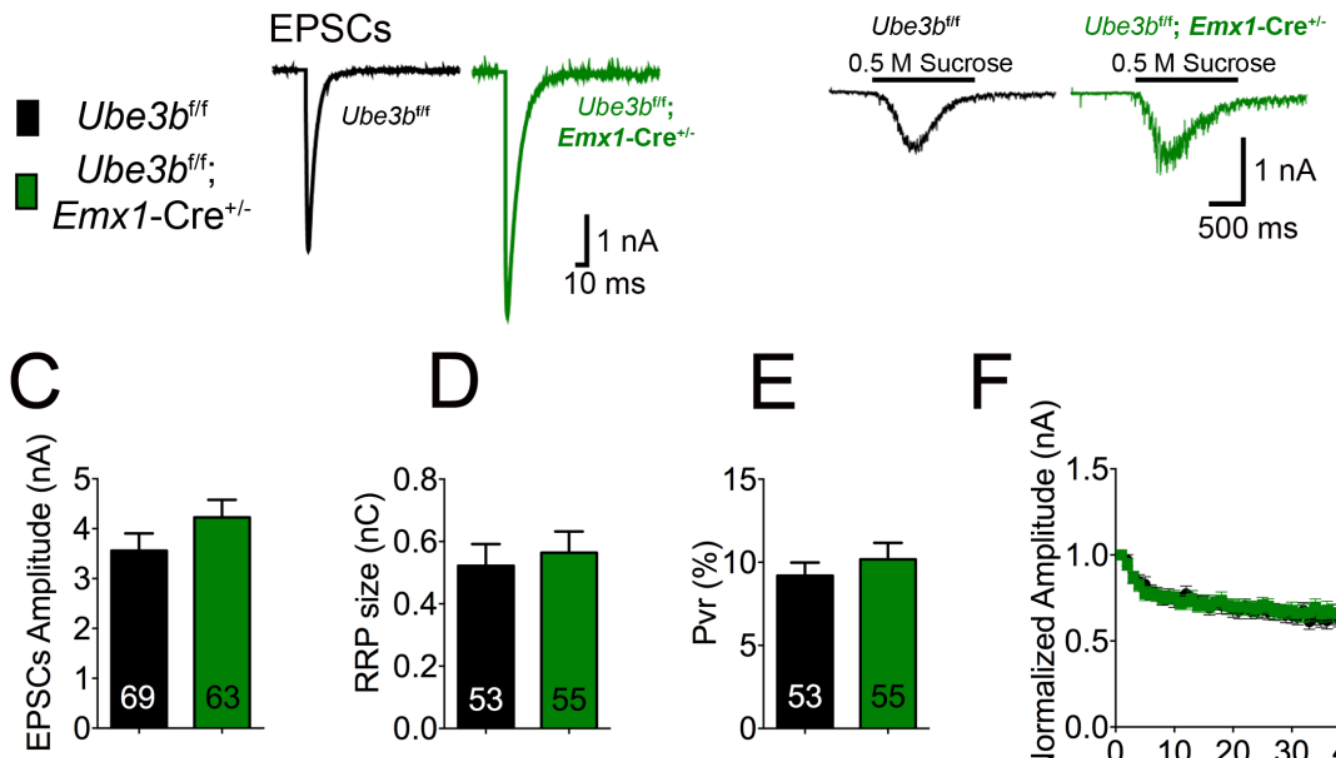

$F$

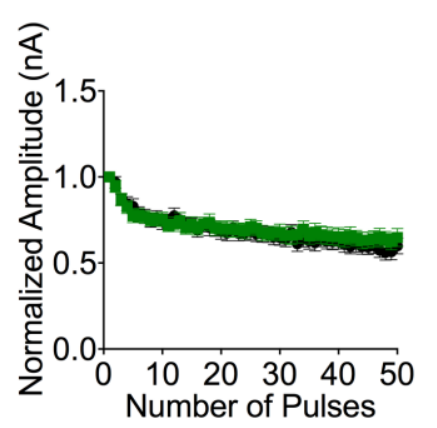

G

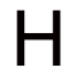

mEPSCs
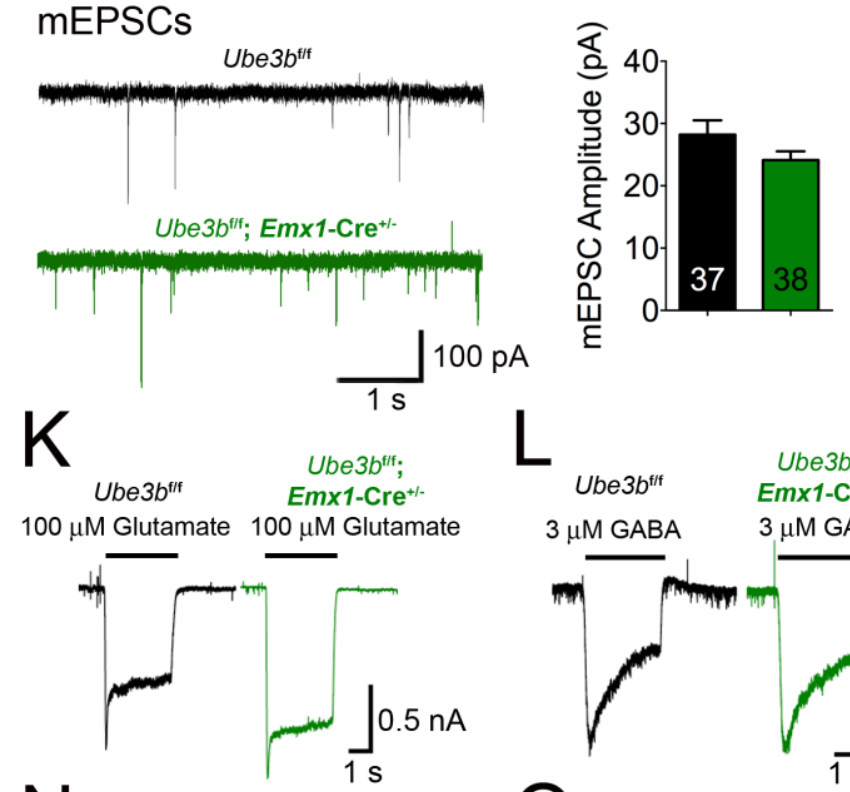

$\mathrm{N}$
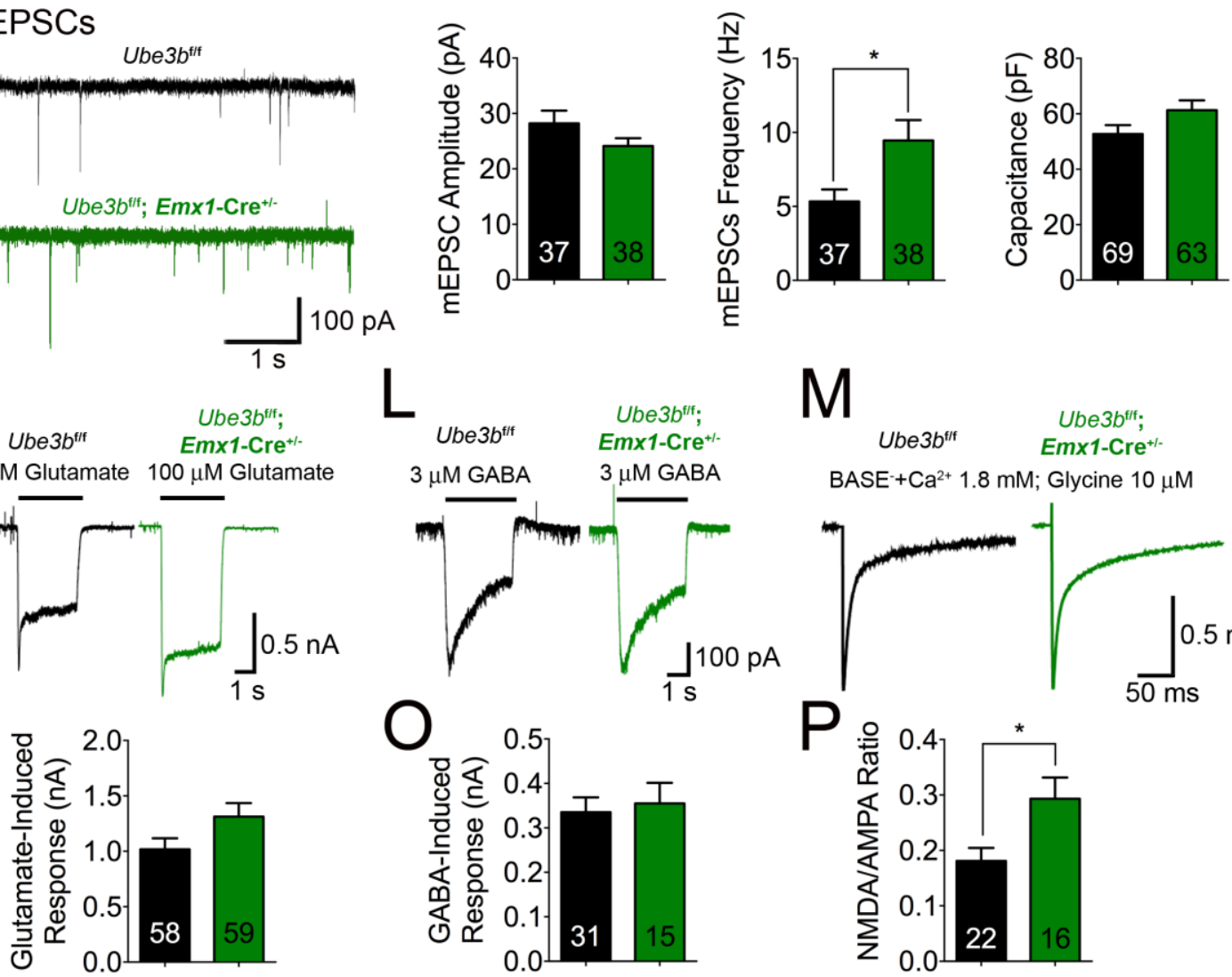

$\mathrm{M}$

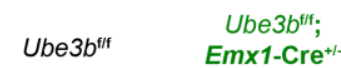

$\mathrm{BASE}^{-}+\mathrm{Ca}^{2+} 1.8 \mathrm{mM}$; Glycine $10 \mu \mathrm{M}$

GABA
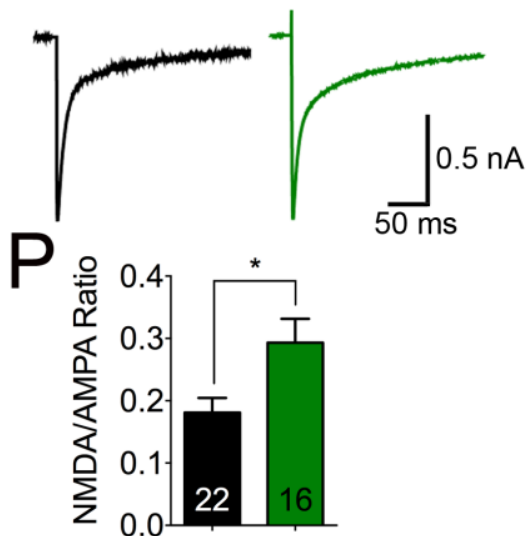

Figure 32. Altered synaptic transmission in $\boldsymbol{U} b e 3 b$ knockout neurons. (A) Sample traces of evoked excitatory postsynaptic currents (EPSCs) from $U b e 3 b^{\mathrm{f} / \mathrm{f}}$ (control) and $U b e 3 b^{\mathrm{f} / \mathrm{f}}$; $E m x 1-\mathrm{Cre}^{+/-}$(Ube3b cKO) autaptic neurons. (B) Sample traces of postsynaptic currents following hypertonic sucrose application in control and $U b e 3 b \mathrm{cKO}$ neurons. (C) Averaged amplitudes of evoked EPSCs from control and Ube $3 b \mathrm{cKO}$ neurons (control, $\mathrm{n}=69 ;$ Ube $3 b$ 
$\mathrm{cKO}, \mathrm{n}=63$ neurons; $\mathrm{p}=0.18$; unpaired t-test). (D) Mean sizes of readily releasable vesicle pool in control and Ube $3 b \mathrm{cKO}$ neurons (control, $\mathrm{n}=53 ;$ Ube $3 b \mathrm{cKO}, \mathrm{n}=55$ neurons). (E) Average release probability in control and $U b e 3 b \mathrm{cKO}$ neurons (control, $\mathrm{n}=53 ; U b e 3 b \mathrm{cKO}$, $\mathrm{n}=55$ neurons). (F) Plots of normalized peak amplitude ( \pm S.E.M) versus number of stimuli at $10 \mathrm{~Hz}$ frequency in control and Ube $3 b \mathrm{cKO}$ autaptic nerve cells (control, $\mathrm{n}=52 ;$ Ube $3 b$ cKO $\mathrm{n}=41)$. (G) Sample traces of miniature EPSCs in control and Ube $3 b$ cKO neurons recorded in the presence of $300 \mathrm{nM}$ tetrodotoxin (TTX). (H) Averaged amplitudes of miniature EPSCs (mEPSCs) in control and Ube $3 b$ cKO neurons (control, $\mathrm{n}=37$; Ube $3 b$ $\mathrm{cKO}, \mathrm{n}=38$ neurons). (I) Averaged frequencies of mEPSCs in control and Ube $3 b \mathrm{cKO}$ neurons. Note almost double increase of the EPSC frequency in Ube3b cKO neurons (control, $\mathrm{n}=37 ;$ Ube $3 b \mathrm{cKO}, \mathrm{n}=38 ; * \mathrm{p}=0.014$; unpaired t-test). (J) Averaged capacitances of control and $U b e 3 b \mathrm{cKO}$ (control, $\mathrm{n}=69 ;$ Ube $3 b \mathrm{cKO}, \mathrm{n}=63$ neurons; $\mathrm{p}=0.08$, unpaired t-test). (K) Sample traces of postsynaptic currents induced by $100 \mu \mathrm{M}$ exogenous glutamate application in control and Ube $3 b$ cKO neurons. (L) Example traces of currents upon application of $3 \mu \mathrm{M}$ GABA in control and Ube $3 b$ cKO neurons (M) Sample traces of postsynaptic currents upon activation of both - NMDARs and AMPARs by $\mathrm{Mg}^{2+}$-free extracellular solution, $1.8 \mathrm{mM} \mathrm{CaCl}_{2}$, and $10 \mu \mathrm{M}$ glycine in control and $U b e 3 b$ cKO neurons. $(\mathrm{N})$ Averaged glutamate induced responses in control and $U b e 3 b \mathrm{cKO}$ neurons (control, $\mathrm{n}=$ 58; Ube $3 b \mathrm{cKO}, \mathrm{n}=59 ; \mathrm{p}=0.06$, unpaired t-test). (O) Averaged GABA-induced responses in control and $U b e 3 b \mathrm{cKO}$ neurons (control, $\mathrm{n}=31 ;$ Ube $3 b \mathrm{cKO}, \mathrm{n}=15$ ). (P) Averaged ratios between current amplitudes through NMDARs and AMPARs in control and Ube $3 b$ cKO neurons (control, $\mathrm{n}=22$; Ube $3 b \mathrm{cKO}, \mathrm{n}=16 ;{ }^{*} \mathrm{p}=0.013$, unpaired t-test). Results are represented as average \pm S.E.M.

\subsubsection{Imbalance of excitatory/inhibitory transmission in the hippocampus of Ube3b cKO mice}

Our findings indicate that Ube $3 \mathrm{~b}$ loss results in increased synaptic strength paralleled by altered spine morphology and increased spine density on glutamatergic hippocampal neurons. This in turn may lead to altered properties of hippocampal circuitry in $U b e 3 b^{\mathrm{f} / \mathrm{f}}$; $E m x 1-\mathrm{Cre}^{+/-}(U b e 3 b \mathrm{cKO})$ animals, affecting the ability of neurons to synchronize and oscillate. In order to validate this hypothesis, with Dr. Silvia Ripamonti and Dr. Matthieu Hammer, we recorded $\gamma$-oscillatory activity from P14-P2 4 in brain slices in CA3 field of hippocampus at the frequency of $25-45 \mathrm{~Hz}$ upon kainate application. Strikingly, inducing $\gamma$-oscillations by application of $100 \mathrm{nM}$ kainate resulted in epileptiform activity in approximately $90 \%$ of slices prepared from $U b e 3 b \mathrm{cKO}$ mice (Fig. 33C). Application of 50 $\mathrm{nM}$ kainate resulted in decreased number of slices with epileptic seizure-like activity,and enabled to record the $\gamma$-oscillations (Figs. $33 \mathrm{~A}$, and 33B). While no changes in the frequency of $\gamma$-oscillations were detected between $U b e 3 b^{\mathrm{f} / \mathrm{f}}$ control and $U b e 3 b \mathrm{cKO}$ animals (Fig. 33D), we observed a trend towards an increase in average power of $\gamma$-oscillations in $U b e 3 b \mathrm{cKO}$ 
as compared to control mice (Fig. 33E). These data indicate that hippocampal circuitry in $U b e 3 b \mathrm{cKO}$ exhibits higher sensitivity to kainate resulting in epileptiform activity.

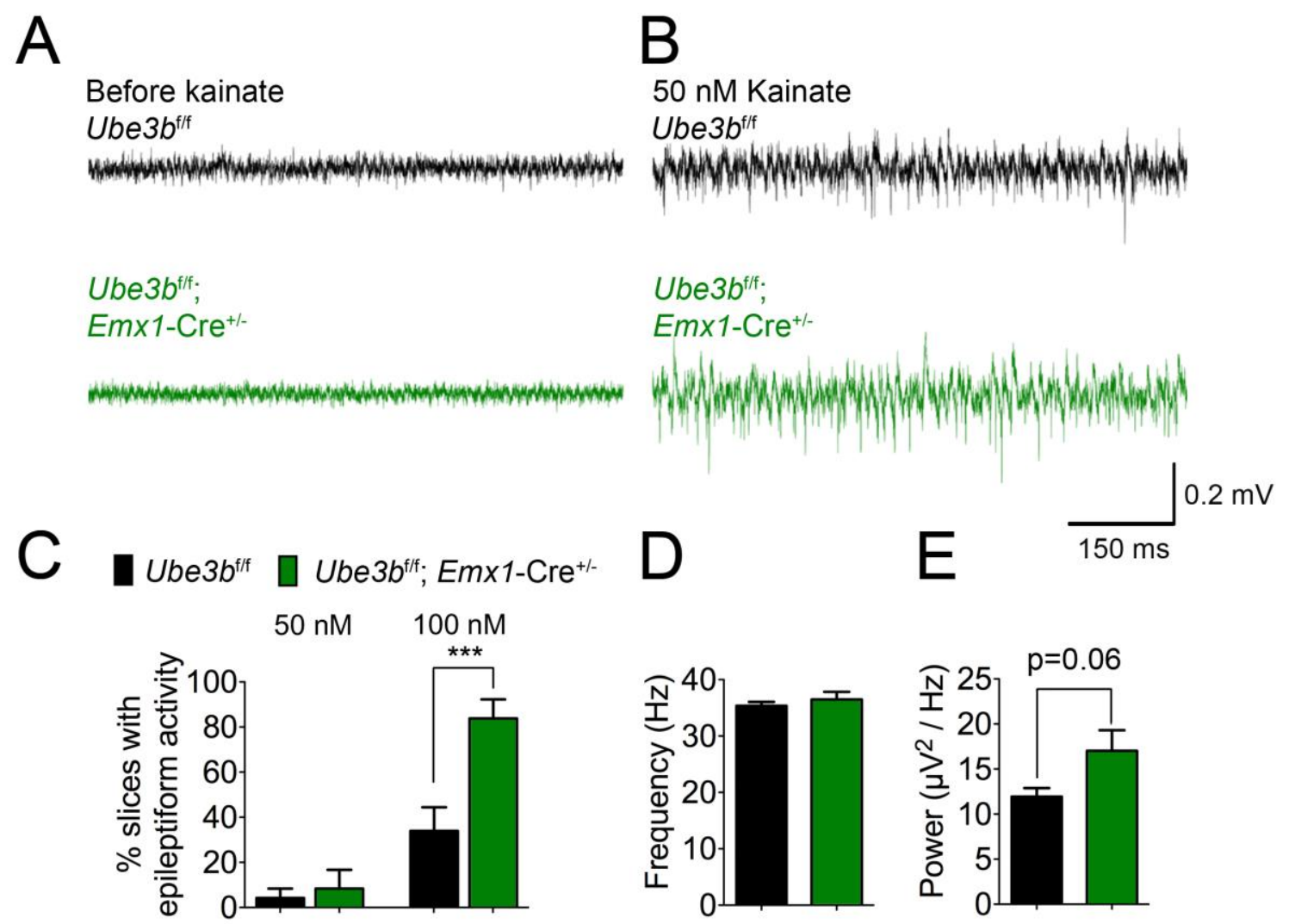

Figure 33. Gamma-oscillations in Ube3b cKO mice. (A, and B) Representative traces of recordings of gamma oscillations before application of kainate (A), and during slice treatment with $50 \mathrm{nM}$ kainate (B) in $U b e 3 b^{\mathrm{f} / \mathrm{f}}$ (top traces, A, and B; control) and $U b e 3 b^{\mathrm{f} / \mathrm{f}}$; Emx l-Cre ${ }^{+/-}$(bottom traces, A, and B; Ube $3 b \mathrm{cKO}$ ). (C) Quantification of the number of brain slices in control and $U b e 3 b \mathrm{cKO}$ mice with epileptiform activity upon 50, or $100 \mathrm{nM}$ kainate treatment. (D) Quantification of frequency of $\gamma$-oscillations in control and Ube $3 b$ cKO mice following $50 \mathrm{nM}$ kainate treatment. (E) Quantification of power of $\gamma$-oscillations in control and $U b e 3 b \mathrm{cKO}$ mice during $50 \mathrm{nM}$ kainate application. All bars represent average \pm S.E.M. Control, $\mathrm{n}=6$ mice; Ube $3 b \mathrm{cKO}, \mathrm{n}=6$ mice; $* * * \mathrm{p}<0.001$, unpaired t-test. 


\subsubsection{Severe loss of spatial memory and disturbances in social interaction in Ube $3 b$ cKO mice}

The disruption of spatial learning in $U b e 3 b$ cKO mice indicates defects in synaptic transmission in hippocampal CA1 region [299]. To analyze the behavioral changes upon neuronal loss of Ube3b, we collaborated with Prof. Ekrem Dere and Prof. Hannelore Ehrenreich (MPIem, Göttingen), who performed a battery of behavioral examinations of $U b e 3 b^{\mathrm{f} / \mathrm{f}}$ (control) and $U b e 3 b^{\mathrm{f} / \mathrm{f}} ; E m x 1-\mathrm{Cre}^{+/-}(U b e 3 b \mathrm{cKO})$ mice. In this thesis, we are presenting the data on spatial memory and social interactions in control and Ube $3 b \mathrm{cKO}$ mice.

First, we performed visible platform test assessing motivation to escape from swimming in the pool as well as the ability to swim and navigate to a visible platform within the pool. The latency to escape onto the visible platform ( $\mathrm{p}<0.001$ for control and Ube $3 b \mathrm{cKO}$, Fig. 34A) and the distance swum until the visible platform was reached $(\mathrm{p}<0.001$ for control and Ube3b cKO, Fig. 34B) was significantly lower on the second as compared to the first day of visible platform training in both control and $U b e 3 b \mathrm{cKO}$, indicating intact procedural learning. The swim speed of control mice was significantly higher on the second as compared to the first day of testing ( $p=0.001$, Fig. $34 \mathrm{C})$. No such effect was observed in the $U b e 3 b \mathrm{cKO}$ mice $(\mathrm{p}=0.124)$, indicating that they were equally motivated to escape from the pool on both days of testing. No significant differences were observed for escape latencies, path lengths, or swim speeds between the two groups. These results depict that Ube3b cKO are sufficiently motivated to escape from the pool, have intact swimming abilities, and can use visual information to navigate to a certain position in space.

Further, we investigated spatial learning measured during the eight days of hidden platform acquisition, where the mice navigated to a platform submerged underneath the water. The latency to locate the hidden platform as well as the distance swum until the mice arrived onto the platform significantly decreased during subsequent days of hidden platform training (main effect of training day, latency: $p<0.001$; path length: $p<0.001$, Fig. 34D, and 31E). No such effect of training day was observed for the swim speed ( $p=0.739$; Fig. 34F). Compared to the control mice, Ube $3 b \mathrm{cKO}$ animals displayed significantly impaired spatial learning. During the eight days of hidden platform training, Ube $3 b \mathrm{cKO}$ mice presented significantly increased escape latencies (main effect of genotype: $p=0.006$ ) and swum significantly longer distance in the pool $(\mathrm{p}<0.001)$ before finding the hidden platform as compared to the control. The spatial learning deficits upon $U b e 3 b \mathrm{cKO}$ were not due to 
changes in swimming ability or swim speed $(p=0.397$, Fig. $34 \mathrm{~F})$. Given that no significant differences between control and $U b e 3 b$ cKO mice were observed in the visible platform test as described above, the spatial learning deficit is not a consequence of secondary changes in the motivation to escape, motor deficits or aberrances of sensory-motor integration. Therefore, Ube3b depletion from glutamatergic neurons of the forebrain and glia leads to severe hippocampus-dependent spatial learning deficit.

A day after the hidden platform training, we tested spatial memory in control and Ube $3 b$ cKO animals with the platform removed from the water-maze. As compared to control, Ube $3 b \mathrm{cKO}$ mice spent less time searching for the platform in the area with previously located platform ( $p=0.128$, Fig. $34 \mathrm{G}$ ) and made significantly fewer visits to the former platform quadrant $(\mathrm{p}=0.020$; Fig. $34 \mathrm{H})$. These results illustrate that $U b e 3 b \mathrm{cKO}$ mice were unable to associate the spatial position of the platform with salient extra-maze cues.

As part of a social behavior-testing paradigm, we then investigated nesting behavior in control and $U b e 3 b \mathrm{cKO}$ mice. There were no significant differences between both groups neither in terms of the quality of the nest built overnight ( $p=0.966$; Fig. 34I), nor in the amount of material used ( $\mathrm{p}=0.868 ;$ Fig. $34 \mathrm{~J})$ to construct the nest. In summary, Ube $3 b \mathrm{cKO}$ seem not to affect nesting behavior in mice.

Next, we analyzed social interactions, where a pair of unfamiliar mice of the same genotype is placed into a familiar environment to measure the propensity to engage in social interactions. Ube $3 b \mathrm{cKO}$ mice during the first five minutes of the test spent significantly more time on social interactions $(\mathrm{p}=0.016$; Fig. $34 \mathrm{~K})$ as compared to the wild type mice. This difference was no longer evident during the last five minutes of the test $(p=0.330$; Fig. $34 \mathrm{~L}$ ), when the animals were already familiar with each other. These results hint that $U b e 3 b$ $\mathrm{cKO}$ in mice leads to an increase in social interactions or sociability.

We then tested for sociability and social memory of control and Ube $3 b \mathrm{cKO}$ in the tripartite chamber. No significant differences between control and Ube $3 b \mathrm{cKO}$ mice was observed during the sociability test, where the animals had to discriminate between a chamber with unfamiliar mouse and a chamber with empty cage (Fig. 34M). Interestingly, while control mice showed a significant preference for the chamber with unfamiliar mouse over the empty chamber $(\mathrm{p}=0.017)$, no such inclination was detected in the Ube $3 b \mathrm{cKO}$ mice $(\mathrm{p}=0.865)$. Moreover, during the social memory test on the third trial the $U b e 3 b \mathrm{cKO}$ performed much better than the control animals and spent significantly more time in the compartment with unfamiliar mouse as compared to the chamber where the familiar mouse was presented $(\mathrm{p}=$ 0.0004; Fig. 34N). Further, Ube3b cKO mice spent significantly more time in the 
compartment where the unfamiliar mouse was located as compared to control $(p=0.003)$. No such difference was observed for the time spent in the chamber where the familiar mouse was located $(p=0.615)$. These findings indicate dissociation between spatial and social memory performance in $U b e 3 b \mathrm{cKO}$ animals. Our results pinpoint that the neural substrates of spatial and social memory are distinct and can be affected independently from each other. In conclusion, the Ube $3 b \mathrm{cKO}$ mice exhibit complex behavioral phenotype with both gain and loss of function, including a facilitated processing of intensive, high-arousal sensory stimuli, behavioral switch towards self-directed actions (both data not shown), impaired spatial learning and memory, but increased sociability, and social memory. 


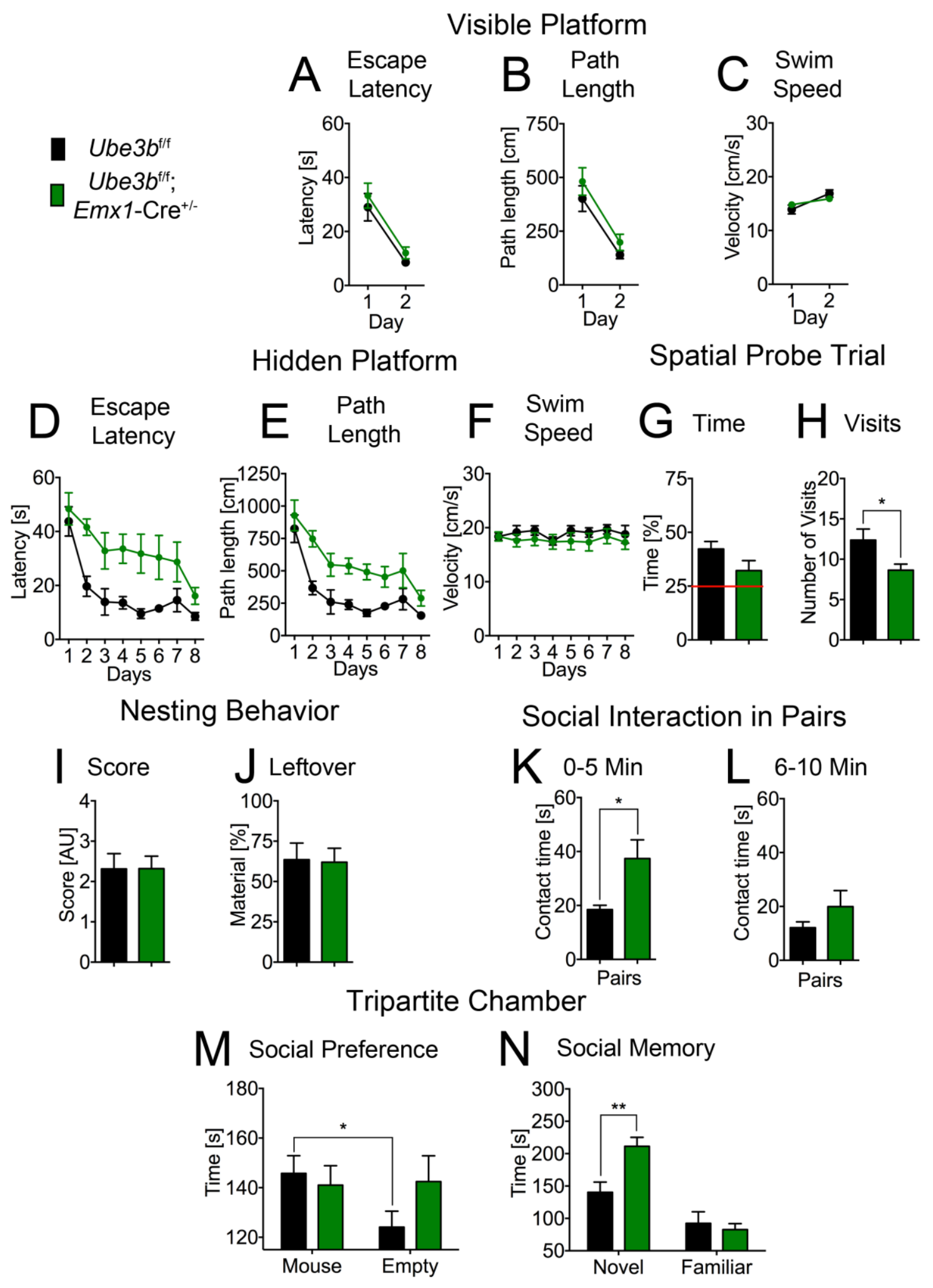

Figure 34. Behavioral testing of $U b e 3 b$ cKO mice. (A) Quantification of escape latencies in control, $U b e 3 b^{\mathrm{f} / \mathrm{f}}$ and $\mathrm{cKO}, U b e 3 b^{\mathrm{f} / \mathrm{f}} ; E m x l-\mathrm{Cre}^{+/-}$mice. Circles represent average \pm S.E.M escape latency ( $\mathrm{s}$ ) to the visible platform on indicated days. (B) Quantification of distances until the visible platform was reached on indicated days. Circles represent mean \pm S.E.M distance swum $(\mathrm{cm})(\mathrm{C})$. Averages of swim speed. Circles represent mean \pm S.E.M swim 
speed $(\mathrm{cm} / \mathrm{s})$ on indicated days. (D) Plot of escape latencies during eight days of Morris Water Maze testing. Circles represent mean \pm S.E.M escape latency (s) to the hidden platform on indicated days. ${ }^{* *} \mathrm{p}=0.006$, Repeated Measures ANOVA. (E) Plot of average distance swum until the hidden platform was reached on indicated days. Circles represent mean \pm S.E.M. ${ }^{* * *} \mathrm{p}<0.001$, Repeated Measures ANOVA. (F) Plot of average swim speed on indicated days. Circles represent mean \pm S.E.M. (G) Average time of control and Ube $3 b$ cKO mice in target zone. Bars represent mean \pm S.E.M \% time spent in the former platform quadrant. Red line indicates performance at chance likelihood. $(\mathrm{H})$ Averages of number of visits a mouse made to former platform quadrant. Bars represent mean \pm S.E.M number swims into the target zone. Unpaired t-test; $* \mathrm{p}=0.02$. (I) Averages of nesting score in control and Ube $3 b \mathrm{cKO}$ animals. Bars represent mean \pm S.E.M. (J) Quantification of the average material used for building a nest. Bars represent mean \pm S.E.M unused nesting material. (K) Social interaction in pairs test. Bars represent mean \pm S.E.M contact time between two unfamiliar mice of the same genotype during the first five minutes of the test. $* \mathrm{p}=0.016$, unpaired t-test. (L) Bars represent mean \pm S.E.M contact time during the last five minutes of the test. (M) Sociability and social memory of control and $U b e 3 b \mathrm{cKO}$ mice was tested in the tripartite chamber. Bars represent mean \pm S.E.M time spent in the unfamiliar mouse and empty compartments. ${ }^{*} \mathrm{p}=0.017$, paired t-test. (N) Bars represent mean \pm S.E.M time spent in the compartments containing an unfamiliar and familiar mouse. $* * \mathrm{p}=0.003$, unpaired t-test. All described test were conducted with males; control, $\mathrm{n}=8$; Ube $3 b \mathrm{cKO}, \mathrm{n}=11$.

\subsubsection{Proteomic screens reveal putative Ube3b substrates}

In the final set of experiments, we studied molecular pathways involving Ube $3 \mathrm{~b}$ that underlie neuronal development. To identify Ube $3 b$ substrates relevant for neurite and spine development, we performed screening using yeast two-hybrid method using N-terminal sequence of Ube $3 \mathrm{~b}$ as a bait and mouse embryo and rat brain cDNA libraries (Fig. 35A). We identified eight proteins as potential binding partners of Ube3b (Fig. 35A). In order to validate the binding between the bait and prey proteins, HEK cells were transfected with a plasmid encoding for myc-tagged Ube $3 b$. The protein extract was loaded to glutathione beads coupled with GST-fused prey proteins identified in the screen. Western blotting with an anti myc-tag antibody demonstrated that Ube3b specifically binds to GST-fused Tacc1, Paip1, Cep120, Celf2, and Ap2b1 but not to negative control, GST (Fig. 35B).

As an alternative approach to identify the substrates of Ube $3 b$, we performed comparative proteomics. The C-terminal structure of the HECT domain specifies the type of polyubiquitin chain conjugated with the substrate protein [300]. Ube3c, whose C-terminus shares high homology to the one of Ube3b, mainly endows the target proteins with Lys48linked polyubiquitin chains [301]. This indicates that Ube3b conjugates Lys48-linked polyubiquitin chains to its substrates, mediating their proteasomal degradation. Given that 
Ube3b is highly enriched at the postsynaptic density fraction (Fig. 27F), we hypothesized local Ube3b-mediated ubiquitylation and accumulate of Ube $3 b$ substrates at the postsynapse in the $U b e 3 b^{\mathrm{f} / \mathrm{f}} ; E m x 1-\mathrm{Cre}^{+/-}$mouse. Based on this hypothesis, to identify Ube3b substrates, we purified synaptic plasma membrane fractions (SM3) from $U b e 3 b^{\mathrm{f} / \mathrm{f}}$ (control) and $U b e 3 b^{\mathrm{f} / \mathrm{f}} ; E m x l-\mathrm{Cre}^{+/-}(U b e 3 b \mathrm{cKO})$ mice and subjected them for comparative mass spectrometry (performed by Olaf Jahn, MPIem, Göttingen).

First, we validated the purification of SM3 fractions by Western blotting with antibodies against PSD-95, and RabGDI (Fig. 35C), and confirmed depletion of Ube3b in SM3 fractions purified from Ube3b cKO mice (Samples M4 to M6 in Fig. 35D). Based on the number and mass spectrum of unique peptides recognized by the spectrometer, we obtained a list of synaptic of proteins up-regulated in SM3 fractions from Ube $3 b \mathrm{cKO}$ as compared to the ones prepared from control mice (Fig. 35E). We then validated the results by Western blotting with the antibody against the top hit protein, protein phosphatase 3 , catalytic subunit, gamma isoform, Ppp3cc (Fig. $35 \mathrm{~F}$ ). The level of Ppp3cc is significantly increased in Ube $3 b$ cKO samples as compared to control samples (control, $1 \pm 0.47$; Ube $3 b \mathrm{cKO}, 3.68 \pm 0.90$; Fig. 35G), indicating that Ppp3cc may be a Ube3b substrate, regulated locally at the synapse. 


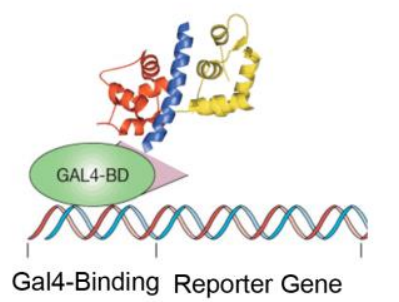

Mouse Embryo

Paip1

Tacc1

Rat Brain

Cep120

CUGBP2

Talin-2

Ap2b1

Git1

Celf2

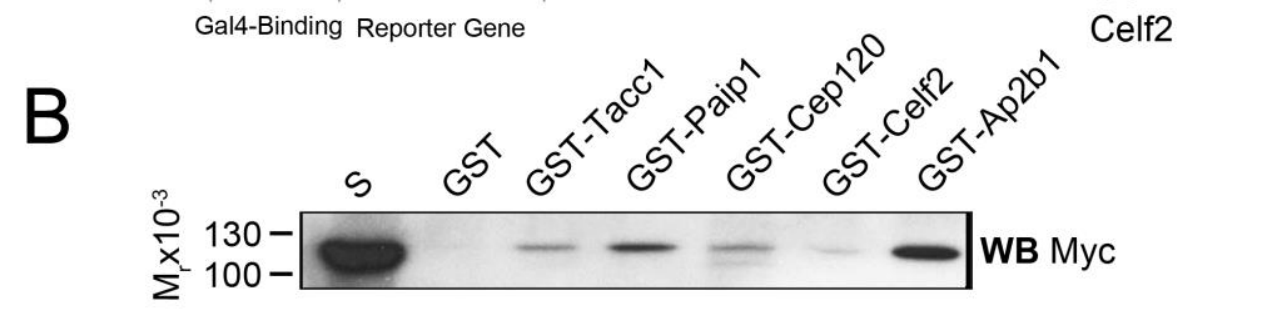

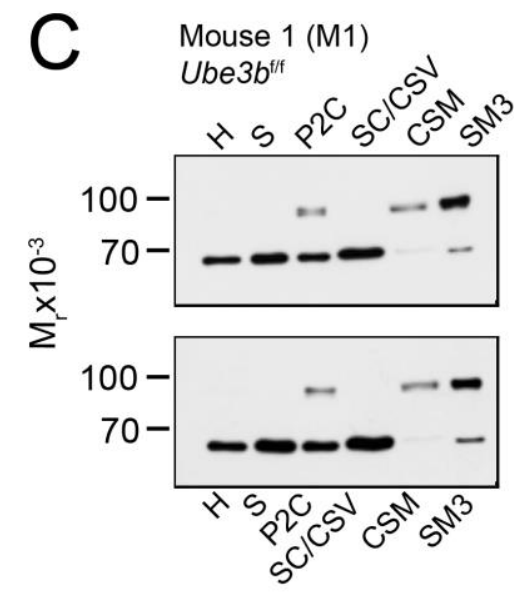

Mouse 4 (M4)

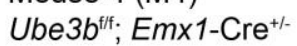

Mouse 2 (M2)
Ube $3 b^{f / 4}$
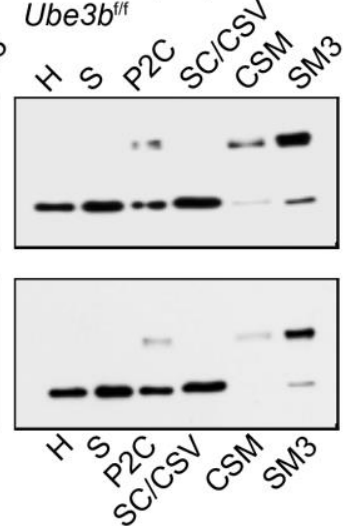

Mouse 5 (M5)

Ube3b ${ }^{\mathrm{fff}} ; \mathrm{Em} \times 1-\mathrm{Cre}^{+/-}$
Mouse 3 (M3)
D

F

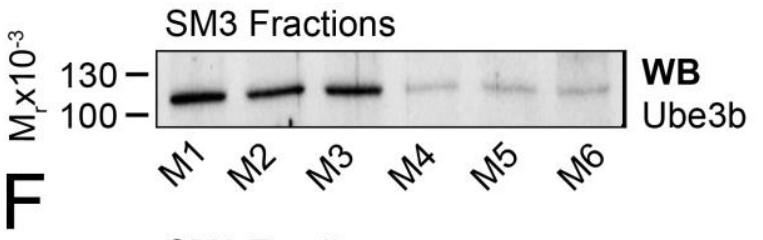

E

\begin{tabular}{ccc}
$\begin{array}{c}\text { Gene } \\
\text { Name }\end{array}$ & $\begin{array}{c}\text { UniProt } \\
\text { Accession }\end{array}$ & $\begin{array}{c}\text { Log2 ratio } \\
\text { WT/KO }\end{array}$ \\
\hline Ppp3cc & P48455 & -1.32 \\
Hide1 & Q75VT8 & -0.98 \\
Rp/36 & P47964 & -0.68 \\
Sept14 & Q9DA97 & -0.65 \\
Actn3 & O88990 & -0.61 \\
\hline Dnajc10 & Q9DC23 & -0.56 \\
Camk2d & Q6PHZ2 & -0.51 \\
\hline
\end{tabular}

WB

$\mathrm{G}$

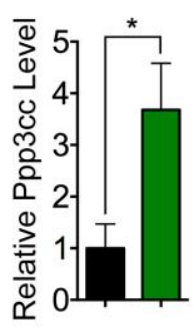

Figure 35. Proteomic screens for Ube3b substrates. (A) The scheme of yeast two hybrid screening for Ube $3 \mathrm{~b}$ binding proteins using N-terminus of Ube $3 \mathrm{~b}$ containing IQ motif as bait (left scheme). The list of putative Ube3b interacting proteins is displayed on the right scheme. (B) Validation of Ube3b binding proteins is displayed on the right scheme. The binding of myc-Ube $3 \mathrm{~b}$ with indicated proteins was confirmed by Western blotting using 
anti-myc antibody. (C) Western blotting validation of subcellular fractionation of $U b e 3 b^{\mathrm{f} / \mathrm{f}}$, control (Mouse $1-3$ ) and $U b e 3 b^{\mathrm{f} / \mathrm{f}} ; \mathrm{Emx} 1-\mathrm{Cre}^{+/}$, Ube $3 b \mathrm{cKO}$ (Mouse 4 - 6) brains using antibodies against PSD-95, and RabGDI. Homogenate $(\mathrm{H})$, supernatant $(\mathrm{S})$, synaptosomes (P2C), synaptic cytoplasm, crude synaptic vesicles (SC/CSV), crude synaptic membrane (CSM), synaptic membrane fraction (SM3). (D) Validation of Ube3b loss in SM3 samples purified from Ube3b cKO (compare also C) by Western blotting. (E) List of proteins upregulated in the SM3 fraction from $U b e 3 b^{\mathrm{f} / \mathrm{f}} ; E m x 1-\mathrm{Cre}^{+/-}$by proteomic screening. (F) Validation of comparative proteomics (compare also D, and E) by Western blotting using SM3 fractions of control and Ube $3 b$ cKO brains with antibodies against the top hit, Ppp3cc (top panel). MEM code staining of nitrocellulose membrane used for Western blotting (bottom panel). (G) Quantification of Ppp3cc levels in control and Ube3b cKO SM3 fractions. Protein level was normalized to MEM Code staining intensity, and expressed relative to Ppp3cc level in the control. Data are represented as averages \pm S.D. Control, $\mathrm{n}=$ 3; Ube $3 b \mathrm{cKO}, \mathrm{n}=3 ; *<0.05$, unpaired t-test. 


\section{DISCUSSION}

\subsection{Wwp ubiquitin ligases, miR-140, and Sox9 regulate neuronal polarity acquisition}

\subsubsection{Wwp1 and Wwp2 are indispensable for axon specification in primary hippocampal neurons}

Wwp1 and Wwp2 ligases have been extensively studied in dividing cells, however their role in postmitotic neurons remain uncharted. During this study, we corroborated essential roles of Wwp1 and Wwp2 in axon acquisition of hippocampal neurons in vitro, polarity formation of cortical nerve cells, and their distribution throughout cortical plate in vivo.

An increase in the fraction of neurons projecting multiple axons upon Wwp1/2 $\mathrm{dKD}$ and upon $W w p 1 / 2 \mathrm{dKO}$ (Fig. 5 and Fig. 8) indicates indispensable roles of Wwp1 and Wwp2 in axon specification. To date, little examples of gene $\mathrm{KO}$ resulting in gain-of-function in axon specification have been reported. Loss of tuberous sclerosis 1 (Tscl), or KD of tuberous sclerosis 2 (Tsc2) induces formation of ectopic axons in primary hippocampal neurons, resembling the phenotypic change in $W w p 1 / 2 \mathrm{dKO}$ and Wwp $1 / 2 \mathrm{dKD}$ neurons [302].

Tsc1 and Tsc2 control axon formation by negatively regulating mammalian target of rapamycin complex 1 (mTORC1) pathway. Interestingly, KD of phosphatase and tensin homolog (Pten), alternative regulator of mTOR pathway, results in a gain-of-function phenotype in axon formation. Pten acts upstream of glycogen synthase kinase $3 \beta$ (GSK3 $\beta$ ), whose siRNA-mediated KD also leads to multiple axons [303].

Our data show that Wwp1 and Wwp2 regulate a molecular pathway essential for the regulation of axon formation in hippocampal neurons.

\subsubsection{Wwp1 and Wwp2 are essential for acquisition of neuronal polarity formation in vivo}

Defectful axon formation upon Wwp1/2 $\mathrm{dKD}$ and $W w p 1 / 2 \mathrm{dKO}$ in vitro might reflect aberrant apico-basal polarity acquisition in progenitor cells, or defectful multipolar-bipolar transition essential for initiation of radial migration (Fig. 6 and 7) [304,305]. We cannot exclude the possibility that Wwp1/2 $\mathrm{dKD}$ might induce changes of neuronal progenitor identity and thereby destines neurons to deeper cortical layers formed earlier in neurogenesis 
[306]. Nonetheless, observed concomitant loss of bipolar morphology in newly born neurons upon Wwp1/2 dKD in vivo implies defects in specification of leading and trailing processes, both of which are critical for establishment of axon/dendrite polarity [232,307].

Given that we do not observe clustering of non-polarized neurons at the ventricular zone, observed for mutations leading to loss of axons, Wwp1/2 $\mathrm{dKD}$ in neurons seem to disrupt the maintenance and not the initiation of radial migration. LKBI $\mathrm{KO}$ abrogates axon formation in neurons and KD of LKB1 results in an ultimate arrest of neuronal migration [230,234,308]. Deletion of transforming growth factor receptor $\beta$ type II, (Tgfr $\beta$ II) in neocortical neurons abrogates axon formation and results in accumulation of nerve cells at the ventricular zone [232]. Presumptively, loss of axon upon LKB1 KO, or Tgfr $\beta I I \mathrm{KO}$ mirrors defective specification of leading and trailing processes and hamper attachment of a neuron to RGC processes, resulting in the delay of migration [309].

\subsubsection{Nedd4 ligases orchestrate neuronal development}

Interestingly, Nedd4 family of ubiquitin ligases display very similar domain architecture with C2-, WW-, and HECT-domains. WW-domains of all members of Nedd4 family recognize similar amino acid motives in substrates and bind proline rich stretches in proteins [310,311]. Intriguingly, deficiencies of each Nedd4 ubiquitin ligase ensue with different consequences for neuronal morphology. Ubiquitin ligase Nedd4-1 is a positive regulator of neurite branching, and is dispensable for axon specification [97,98]. KD of Smurf1 abrogates axon formation in Par6- and RhoA-dependent mechanisms [96]. Here, we report that loss of $W w p 1$ and $W w p 2$ in neurons leads to specification of multiple axons. Diverse role of Nedd4 family of E3 ligases implicate that despite highly homologous amino acid sequences, Nedd4 ligases ubiquitylate discreet sets of substrates and mediate different cellular signaling pathways controlling different aspects of neuronal development.

\subsection{4. miR-140 acts synergistically to Wwp1 and Wwp2 ligases in regard to axon acquisition}

We demonstrate that miR-140 is indispensable for uncompromised neuronal development. Genetic deletion of miR-140 leads to induction of ectopic axons in primary hippocampal neurons. Exogenous delivery of miR-140 to $m i R-140^{-/-}$neurons restores specification of single axons (Fig. 11), indicative of cell-autonomous mode of miR-140 action in neurons. 
Conversely, expression of tandemly repeated pri-miR-140 in wild type neurons leads to loss of axon specification (Fig. 17). Overexpression of a given miRNA might cause RISC saturation and induce phenotypic artifacts attributable to loss of other miRNAs associated with RISC [273,312]. However, miR-140 overexpression leads to the opposite phenotypic change as $m i R-140 \mathrm{KO}$. Therefore, it is likely that miR-140 suppresses axon formation.

Intriguingly, aberrances of axon specification in vitro, defectful morphology of neurons in vivo, and disruption of cortical distribution is similar for Wwp $1 / 2 \mathrm{dKD}, W w p 1 / 2 \mathrm{dKO}, m i R-$ $140 \mathrm{KO}$, and miR-140-3p KD (Compare Figs. 5, 6, 8, 11, 12, and 16). These observations imply that $m i R-140$ acts synergistically to its host gene $W w p 2$ and homologous $W w p 1$ in regulation of neuronal polarity formation. In the future, it would be interesting to study the combined effects of $m i R-140$ and $W w p l / 2$ loss on neuronal morphology by knocking down miR-140 in $W w p 1 / 2 \mathrm{dKO}$, or knocking down Wwp1/2 in $m i R-140 \mathrm{KO}$.

MiR-Base (GRCm38) as of June 2014 reports that 1915 mature murine miRNAs have been annotated. Some of them have been reported as critical regulators of neuronal development and function, e.g. miR-9 [313], miR-124 [314], miR-132 [315], or miR-137 [316]. However, none of annotated miRNAs has been reported indispensable for polarity formation before, placing miR-140 among the regulators of axon specification in neurons.

Interestingly, genetic deletion of Dicer in neurons leads to disruption of neuronal migration in vivo, paralleled with loss of bipolar morphology of migrating neurons. Loss of Dicer results in pleiotropic neuronal phenotype due to the loss of miRNA processing. Interestingly, expression of mature miR-124 in Dicer KO neurons restores their bipolar morphology [176]. Similarly, genetic deletion of miR-140 disrupts polarity of newly born neurons and affects distribution of cortical nerve cells (Fig. 12). Thus, miR-140 and miR-124 are both involved in regulating polarity formation in nerve cells.

\subsection{5. miR-140-3p acts as a biologically active guide strand to regulate neuronal polarity}

It has been reported, that miR-140 is highly expressed in cartilage during zebrafish ontogenesis [317]. The role of miR-140 has been well characterized in chondrocytes, where miR-140-5p represents more biologically active, guide strand of miR-140 duplex [201,318,319]. Intriguingly, systematic analysis of miRNA expression in neurons using miRNA-tagging and affinity purification method revealed that miR-140-3p strand is expressed in nerve cells, and by far outnumbers miR-140-5p [200]. 
Here, we show that it is the miR-140-3p that is up-regulated in the brain during embryogenesis, and reaches high levels at perinatal stages, when neurons establish axondendrite polarity (Fig. 10D). Moreover, we demonstrate that sponge-mediated downregulation of miR-140-3p leads to ectopic formation of multiple axons in neurons, and that miR-140-5p is dispensable for axon specification (Fig.13).

In situ hybridization using locked nuclei acid experiments illustrate that miR-140-3p is expressed in primary hippocampal neurons, where it enriches in somatic endosome-like structures (Fig. 10E). This finding is in line with somatic localization of miRNA-loaded RISC, and its association with multivesicular bodies, critical for miRNA-mediated gene regulation [320,321]. This indicates that miR-140-3p regulates axon acquisition through global repression of target genes rather than through local suppression of mRNA translation [322].

Our observations suggest that RISC loading complex specifies a guide strand of miR-140 in tissue-specific manner; miR-140-5p in chondrocytes, and miR-140-3p in neurons. Tissuespecific strand selection of miR-140 broadens its regulatory capacity of transcriptome in the entire organism, as each strand targets different pools of mRNAs [200].

Upon RNA sponge-mediated miR-140-3p KD, primary hippocampal neurons, apart from profound defects in axon specification, display high density of conspicuous membrane protrusions, morphologically resembling filopodia (Fig. 13C). Given that filopodia formation relies on precise regulation of actin cytoskeleton [323], and that destabilization of actin filaments is sufficient to induce multiple axons [57], it is likely, that miR-140 is a potential regulator of actin stability in neurons.

Alteration of neuronal distribution within the cortical plate upon miR-140-3p KD in P10 brains (Fig. 15) might be attributable to miR-140-mediated regulation of homeobox genes controlling laminar organization of the cortex. We demonstrate that vast majority of miR140-3p KD neurons abnormally located in deeper cortical layers expressed the marker of layer II/III/IV neurons, Cux1 [29] (Fig. 16). Electroporated cortical progenitors at E14.5E15.5 give rise to layer II/III neurons, thus implicating that miR-140 does not affect expression of homeobox genes. It is therefore likely, that aberrant distribution of miR-140$3 \mathrm{p} \mathrm{KD}$ neurons is due to profound loss of polarity (Fig. 15D), which impedes their migration during development. 


\subsubsection{Neuronal polarity formation is not controlled by reciprocal regulation of miR-140 and Wwp1/2}

In this study, we illustrate that miR-140 acts synergistically with Wwp1 and Wwp2 in neuronal development. To verify if miR-140 and Wwp ligases constitute a reciprocal regulatory loop, such as for miR-26b and its host c-terminal domain small phosphatase 1 , ctdsp2 gene [257], we sought levels of Wwp1 and Wwp2 upon miR-140 KO, and for levels of miR-140 upon $W w p 2 \mathrm{KO}$ (Fig. 18). We report that neither loss of host gene $W w p 2$ causes changes of miR-140 levels, nor deletion of miR-140 affects Wwp1 and Wwp2 protein expression levels in mouse brain. These data excludes reciprocal interaction between miR140 and Wwp ligases, and implies that those molecules operate in the cell in an independent, yet intertwined manner in regulating neuronal polarity acquisition.

\subsection{7. miR-140 regulates $m R N A s$ of proteins involved in polarity acquisition in neurons}

To extend our understanding of molecular context of miR-140-mediated regulation of neuronal development, we sought miR-140 target mRNAs in mouse cortex. By quantitative mass spectrometry, we identify NFM, and Fyn as potential miR-140 targets, involved in polarity formation in developing neurons (Fig.19).

Western blotting validation of quantitative mass spectrometric screening revealed a not statistically significant increase in expression levels of Fyn and NFM in $m i R-140^{-/-}$ synaptosomes, as compared to $m i R-140^{+/+}$samples (Fig. 19E and 19F). Fyn is essential for transduction of semaphorin $3 \mathrm{~A}$ signals to developing neurons, and thereby for proper orientation of apical dendrites in developing cortex [231,307]. NFM is a critical regulator for neuronal morphology by modulating cytoskeleton stability [324]. Moreover, NFM seems to regulate radial growth of the axon [325]. To verify, if Fyn, or NFM is indeed involved in miR-140-mediated control of neuronal polarity acquisition, the morphological consequences of overexpression of both proteins need to be assessed in primary hippocampal neurons as well as in cortical nerve cells.

Mass spectrometry has been previously successfully applied to identify miRNA targets [326,327]. Spectrometric analyses of changes in proteome induced by transfection of HeLa cells with miR-155 reveal that roughly $70 \%$ of putative target genes were down-regulated 
by approximately 30\% [328]. This implies that in general, miRNA-mediated gene regulation mostly relies on fine-tuning gene expression.

A single miRNA molecule is predicted to target from 200 to 300 different mRNAs. Conserved miRNA-binding sites are present in $60 \%$ of human protein-coding genes [146]. It has been proposed, that the phenotype observed upon deletion of a certain miRNA molecule is the additive effect of up-regulation of its several mRNA targets, as is the case for miR-128, or miR-137. CamKII-Cre-driven miR-128-2 cKO leads to early onset hyperactivity, and strong tonic-clonic seizures in mice resulting in death. Interestingly, miR128 deletion leads to up-regulation of ERK2 signaling pathway, however ERK2 kinase is not a direct miR-128 target. Over-activation of ERK2 signaling cascade is due to upregulation of direct miR-128 targets. Indeed, pharmacological inhibition of ERK2 in miR128-2 cKO improves defectful motor performance in mice [329].

Single nucleotide polymorphisms in $m i R-137$ gene have been described in schizophrenic patients and have been reported to elevate miR-137 levels. Interestingly, miR-137 controls the expression of genes that encode proteins of crucial importance for the presynaptic vesicle release in neurons, such as complexin $1(\mathrm{Cplx} 1)$, N-ethylmaleimide sensitive fusion protein (NSF), synapsin III (Syn3), and synaptotagmin 1 (Syt1). Interestingly, viral overexpression of miR-137 in the murine dentate gyrus disrupts vesicle pool at the presynaptic terminals of mossy fibers. Exogenous delivery of miR-137 sponge to neurons induced from fibroblasts of schizophrenic patients restored levels of Cplx1, NSF, and Syt1 and improved vesicle trafficking at the presynapse. Additionally, overexpression of only one of identified miR137 targets, Syt1, was able to restore the deficiencies observed upon miR-137 overexpression in neurons only partially [316].

Both reports corroborate, that phenotypic changes observed upon miRNA deletion, or overexpression should be considered as the broad effects, that miRNA exert on the entire transcriptome, rather than attributing them to miRNA-mediated translation inhibition of a single mRNA.

\subsubsection{Sox9 regulates axon specification, polarity formation, and cortical distribution of postmitotic neurons}

Expression of Sox9 has been previously reported in dividing cells [172,258,274]. In the developing brain, Sox 9 mRNA and protein are developmentally downregulated with the highest expression level during neurogenesis (E13) (Fig. 20A, and 20B). Intriguingly, 
postmitotic hippocampal and cortical neurons of layer II/III displayed nuclear Sox9 expression, indicative of Sox9 roles in neurons (Fig. 20C, and 20D). Wwp2 expression resembles the one of Sox9, both at the levels of mRNA, and protein. This implies, that Sox 9 presumptively operates as a major transcriptional regulator of Wwp2 in developing cortex. Wwp1 protein expression is inversely correlated to the one of Sox9, indicative of other means of transcriptional control of Wwpl gene. Wwp1 and Wwp2 mRNA levels peak perinatally. Additionally, cortical level of miR-140-3p also increases at birth (Fig. 10D). This suggest that at P0, developmental stage when axons, and dendrites are specified in the mouse cortex, Sox 9 is the main regulator of both $W w p 1, W w p 2$, and precursor miR-140 expression (Fig. 20A and 20B).

We also observe that the mRNA and protein expression profiles of $W w p l$ are uncorrelated over the course of cortical development. Expression of Wwp1 mRNA is induced during neurogenesis, peaks at P0 and decays until P21 as shown by qRT-PCR using primers complementary to the middle part of Wwp1 mRNA (NM_177327.3) (Fig. 20A). On the other hand, Western blotting for Wwp1 using an antibody raised against C-terminal HECT domain showed that Wwp1 protein is developmentally up-regulated, and increases constantly until birth, where it reaches the plateau phase (Fig. 20B). It is possible, that Wwp1 mRNA detected in our qRT-PCR is not an isoform that encodes for Wwp1 protein detected in our Western blotting experiments. Alternatively, Wwp1 mRNA undergoes complex posttranscriptional regulation and drives the expression of Wwp1 protein independently of its mRNA levels [330].

We demonstrate that KD of Sox9 in postmitotic hippocampal neurons induces formation of multiple axons and that overexpression of Sox 9 abrogates axon formation. Genetic deletion of Sox9 leads to early embryonic lethality with profound defects of neural crest [276,331]. For this reason, to verify involvement of Sox9 in the regulation of neuronal polarity in vivo, we took advantage of sh-RNA mediated KD and Cre-induced deletion of Sox 9 using in utero electroporation in Sox $9^{\mathrm{f} / \mathrm{f}}$ mice (Fig. 20D, 22 and 23). Both KD and KO of Sox9 in cortical progenitors led to significant alteration of neuronal distribution within cortical plate. Moreover, Cre-driven deletion of $\operatorname{Sox} 9$ in single neurons induced abnormal neuronal polarity in vivo. These data indicate that Sox9 operates in postmitotic neurons, where it is essential for polarity formation in neurons. 


\subsubsection{Sox9/Wwp $1 / 2 / \mathrm{miR}-140-3 p$ axis is critical for regulation of neuronal development}

In this study, we decipher regulatory mechanisms of neuronal polarity acquisition. We demonstrate that miR-140 is indispensable for neuronal development, and acts synergistically to Wwp1 and Wwp2 ligases to suppress axon acquisition. Moreover, we unveil that Sox9 is critical for the development of the nerve cell. Although more experimental evidence is necessary to verify if miR-140 and Wwp1/2 act within one cellular pathway, and if Sox 9 acts as a master regulator of $m i R-140$ and $W w p 1 / 2$ expression in neurons, we conclude here that Sox9, miR-140, and Wwp1/2 represent intertwined molecular quartet orchestrating neuronal polarity formation.

\subsection{HECT-type ubiquitin ligases and dendritic spines}

\subsubsection{Wwp1 and Wwp2 ubiquitin ligases are potential negative regulators of synaptogenesis in cortical and hippocampal neurons}

Expression patterns of Wwp ligases, especially of Wwp1, suggest their critical role in mature neurons of the murine nervous system. We report that neuron-specific $\mathrm{KO}$ of $W w p l$ and Wwp2 leads to increased spine density in cortical neurons, enlarged spine heads of mushroom spines, and reduced length of filopodia in CA1 pyramidal nerve cells (Fig. 24). Increased density of dendritic spines implicates that Wwp1 and Wwp2 negatively regulate synapse number, either inhibiting synaptogenesis, or augmenting synapse elimination. Synapse number in murine brain reaches maximum at P21. As animals progress in adolescence (after P21), synapses formed during postnatal synaptogenesis stabilize, and due to synapse elimination, synaptic contacts undergo refinement $[46,332,333]$. Given that we quantified spine densities in brains of P21 mice, it is likely that Wwp1 and Wwp2 inhibit synaptogenesis.

According to Fiala and co-workers, changes of spine head size, or spine length affect electrophysiological properties of postsynaptic neurons [334]. Spines with enlarged heads represent more stable synaptic contacts, and harbor more glutamate receptors resulting in increased local conductivity [335,336]. Additionally, reduction in the length of filopodia suggests reduced motility of immature spines in $W w p 1$ and $W w p 2$ dcKO neurons [337]. For this reason, Wwp1 and Wwp2 might suppress synaptogenesis by reducing motility of 
developing spines [334]. Electrophysiological measurements of amplitudes of evoked EPSCs and the frequency of mEPSCs would help to understand the functional consequences of morphological alterations of dendritic spines in $W w p 1$ and $W w p 2$ dcKO neurons.

\subsubsection{Nedd4-2 regulates dendritic spine morphology}

Previous reports demonstrating Nedd4-2-dependent ubiquitylation of sodium channels, and membrane transporters imply that Nedd4-2 regulates neuronal physiology. Moreover, Nedd4-2 gene comprises a potential candidate susceptibility gene for epileptic photosensitivity in patients with idiopathic generalized epilepsy [283]. Here, we demonstrate that Nedd4-2 deletion from neurons leads to morphological changes in spine shape, i.e. longer spines, enlarged heads of thin spines, and reduced density of mushroom-type spines at the expense of bifurcated-type spines (Fig.25).

Spine head size determines the area of postsynaptic density and defines the vesicle pool of its presynaptic bouton [338], suggesting augmented synaptic transmission mediated by the synapse it specifies. However, longer spines, observed in Nedd4-2 cKO may affect calcium distribution within the spine, and thereby reduce activation of the postsynaptic terminals [339]. Excitatory postsynaptic potential amplitudes are inversely correlated with spine lengths [340].

Spine head bifurcation has been observed after tetanic stimulation of neuronal afferents to induce LTP. Activity-dependent segmentation of postsynaptic density apposed to a single presynaptic terminal has been proposed to facilitate synaptic potentiation [341,342]. Moreover, longer spines have small, or negligible contribution to action potentials. However, upon high-frequency stimulation are able to undergo plastic morphological changes and functional plasticity [340]. Presumptively, two phenotypes observed for Nedd4$2 \mathrm{cKO}$, longer spines and bifurcated spine heads, might affect induction, and/or maintenance of LTP. Electrophysiological characterization of Nedd4-2 $\mathrm{cKO}$ neurons using protocols to induce LTP is essential to understand the physiological consequence of morphological alterations.

HECT-type ligases of Nedd4 family, Wwp1, Wwp2 and Nedd4-2 share similar domain organization (Fig. 3C). Highly homologous WW-domains recognize proline stretches in substrate proteins, which suggests functional redundancy of Nedd4 ligases in terms of substrate ubiquitylation [343]. However, our data indicate that Wwp1, Wwp2, and Nedd4-2 
ligases are involved in the regulation of independent cellular pathways controlling dendritic spine morphology.

\subsection{Ube3b, Kaufman oculocerebrofacial syndrome, and the molecular pathologies in intellectual disability}

\subsubsection{Neuron-specific loss of Ube3b in mice recapitulates neurological defects of Kaufman oculocerebrofacial syndrome}

Over the course of this study, we characterized $U b e 3 b$ in the developing and mature brain. Recent reports demonstrate that missense, and point mutations in $U B E 3 B$, gene encoding for ubiquitin ligase E3B lead to Kaufman oculocerebrofacial syndrome (KOS) in humans $[136,137]$. KOS is a developmental disorder with growth arrest, severe intellectual disability, and characteristic facial dysmorphisms. In order to reveal the roles of Ube $3 b$, we generated conventional, and neuron- and glia-specific conditional Ube3b KO mice. Conventional KO of $U b e 3 b$ gene in the mouse recapitulates major aspects of human KOS (Fig. 26). Therefore, our Ube3b KO mouse line can serve as a model of the human KOS.

\subsubsection{Ube3b associates with postsynapses}

Ube3b mRNA was highly expressed in the developing cortical plate and ganglionic eminence at E16, indicating critical roles of Ube3b in neuronal development. In the adult brain, Ube3b mRNA was abundantly expressed throughout the cerebral cortex, hippocampus and brain stem (Fig. 27A). This mRNA expression pattern resembles mRNA distribution of $U B E 3 A, M e C P 2$, or $P Q B P-1$, genes mutated in patients with mental retardation, and therefore essential for uncompromised development and neuronal function [344-346]. Expression profile of Ube3b protein over the course of cortical development (Fig. 27B and 27C) resembles the ones of synaptic proteins, such as PSD-95, SAP102, or neuroligin-1 [281,347]. In line with this observation, we report massive enrichment of Ube3b in postsynaptic density fraction (PSD). This enrichment was even more pronounced than that of PSD-95 [348,349] (Fig. 27F). Our data indicates that Ube3b has essential roles at the postsynapses, and emerges as a key regulatory molecule involved in the pathology of intellectual disability syndrome. 


\subsubsection{Ube3b is a positive regulator of neurite branching}

We demonstrate that deletion of $U b e 3 b$ results in deficient neurite development, which may underlie the development of intellectual disability in patients with KOS. Ube3b is essential for proper neurite branching, and regulates it a cell-autonomous manner (Fig. 28). Moreover, expression of pathogenic point mutants of UBE3B (i.e. G779R and R997P) in Ube3b cKO neurons failed to restore proper neurite branching (Fig. 29). Both mutations have been reported in homozygous configuration in KOS patients suffering from severe developmental delay, and absent speech. Intriguingly, both mutations localize to the HECT domain of UBE3B, implicating detrimental effects on its catalytic function.

Remarkably, we noted that HA-hUBE3B R997P displayed a perinuclear localization in nerve cells at DIV7, while wild type HA-hUBE3B and HA-hUBE3B G779R distributed uniformly in the neuron (compare Figs. 29D and 29F). We conclude, that arginine residue at position 997 is essential for proper localization of hUBE3B in neurons.

\subsubsection{Loss of Ube3b deficiency leads to increase in spine density, enlarged spine heads and longer spines in hippocampal neurons}

We demonstrate that genetic deletion of $U b e 3 b$ leads to aberrances in spine density and alterations of spine morphology (Fig. 31). CA1 pyramidal neurons of Emxl-Cre-driven Ube $3 b \mathrm{cKO}$ mice display significant increase in spine density paralleled by significant enlargement of heads of thin spines (Fig. 31A, 31B, 31D and $31 \mathrm{E}$ ). Deletion of $U b e 3 b$ from individual CA1 pyramidal neurons recapitulates $U b e 3 b \mathrm{cKO}$ phenotype with a dramatic increase in spine density, enlarged spine heads, and longer spines (Fig. $31 \mathrm{C}, 31 \mathrm{~F}-31 \mathrm{H}$ ). Given low transfection efficiency by in utero electroporation, these data suggest a cellautonomous function of Ube $3 \mathrm{~b}$ in the regulation of spine number and morphology.

Our morphometrics of dendritic spines were performed with STED nanoscopy. Given that resolution of STED is $30-60 \mathrm{~nm}$ [350] as compared to approximately $200 \mathrm{~nm}$ of a confocal microscope [351], STED is more suitable for spine morphometrics and enables detection of subtle, yet significant changes of spine morphology [352]. 


\subsubsection{Neuronal morphology and intellectual disability}

\subsubsection{Defective neurite branching in intellectual disability syndromes}

Pathophysiology of intellectual disability is not entirely understood, however mutations in genes resulting in cognitive defects provide a possibility to reveal its cellular and molecular fundaments.

Interestingly, disturbances of neuronal morphology concerning dendritic and axonal branching have been previously reported for numerous genes involved in the pathologies of intellectual disability syndromes. Pyramidal neurons of Angelman syndrome mouse model display morphological deficiencies concerning apical dendrite outgrowth, and exhibit undeveloped, stunted dendrites [120]. Mutations in polyglutamine-binding protein 1 gene, $P Q B P-1$, described in approximately ten cases of familial $\mathrm{X}$-linked mental retardation diseases, abrogate dendritic branching of mouse cortical neurons [289,353,354]. Reduced complexity of dendritic arbor was reported in mouse models of Rett syndrome, deleterious for methyl-CpG-binding protein 2, MeCP2 [355,356].

Moreover, morphological deficiencies in neurons are also common cellular pathology in autism spectrum disorders. Post mortem studies in patients with Rett syndrome, a rare form of autism characterized by developmental delay and intellectual disability, reveal reduced dendritic branching of hippocampal neurons [357].

Proper neurite branching is essential for synaptic integration [358]. The geometry of dendritic tree affects firing patterns of action potentials [359], and the dendritic arbor defines neuronal contribution to network activity and synaptic inputs [360,361]. Therefore, deficient neurite arborization in neurons deleterious for $U b e 3 b$ may contribute to cognitive incapacity of KOS patients.

\subsubsection{Dendritic spine abnormalities and mouse models of intellectual disability syndromes}

In mental retardation, structural changes in neuronal morphology, i.e. dendritic tree arborization and alteration of spine density are causative of altered number of synaptic connections, or inappropriate connectivity [362]. Geert Ramakers proposed so called 'network hypothesis' which states that aberrant development and plasticity of synaptic circuits constitutes the primary cause of mental retardation [363,364]. Importantly, synaptic 
activity is linked to dynamic changes of spine shape, size, and numbers. The plasticity of spine numbers and their morphology is strongly associated with learning [335,337,365-368]. For this reason, morphological changes of dendritic spines in $U b e 3 b$-deficient neurons might lead to disrupted synaptic activity and altered network connectivity, or function.

Mouse models of disorders characterized by intellectual disability present spine abnormalities, which are linked to disturbances of neuronal network connectivity $[334,362]$. Genetic model of Down syndrome, mice with segmental trisomy 16 (Ts65Dn mice) [369], mimic the imbalance of gene dosage on human chromosome 21q21-22.3. Ts65Dn mice exhibit decreased spine numbers and enlarged spine heads on the basal dendrites of CA1 neurons and layer II/III pyramidal neurons, as reported by several independent research groups [370,371]. Decreased spine density in CA1 neurons and motor cortex was also noted for mouse models of Rett syndrome. Mice with mutations in $\mathrm{MeCP} 2$ gene exhibit lower spine density, decreased spine heads size, and increased neck lengths [355,372] paralleled by altered dendritic spine distribution [373].

Fragile X syndrome is the most commonly inherited form of mental retardation [374]. On the contrary to both Down and Rett syndrome mouse models, KO of Fragile X mental retardation 1 gene, FMR 1 in mice results in increased number of spines in layer $\mathrm{V}$ pyramidal neurons and hippocampus $[375,376]$.

Those observations indicate that cellular pathologies in intellectual disability syndromes differ one from another, however all seem to converge on alteration of the structure, or number of dendritic spine. Ube3b deficiency in neurons results in increase in spine number, rather than in impairment of spine maturation. Association of Ube $3 b$ with postsynaptic sites implies local regulation of actin cytoskeleton at the synaptic boutons by the ligase. Altered morphology of spines of each type (enlarged heads and longer spine necks, Fig. 31E, 31G and $31 \mathrm{H}$ ) advocates Ube $3 \mathrm{~b}$-mediated changes of plasticity at the level of single spines, rather than a global switch in maturation of synaptic contacts.

\subsubsection{Deletion od Ube3b leads to increased synaptic transmission and alters NMDAR to AMPAR ratio}

In line with increased spine numbers in $U b e 3 b \mathrm{cKO}$ neurons, electrophysiological analyses of nerve cells devoid of Ube $3 b$ reveal increased mEPSCs frequency without changes in amplitude, indicative of increased numbers of functional synapses specified by Ube $3 b$ neurons (Figs. 32G-I). 
Additionally, we report increased NMDAR to AMPAR ratio in Ube3b cKO neurons (Fig.32P). This alteration indicates either changes in the abundance of glutamate receptors on $U b e 3 b$ cKO neurons, or altered composition of NMDARs facilitating increased NMDAR conductance. Neuronal NMDAR currents are positively correlated with the diameter of the spine head [336]. We therefore hypothesize, that enlarged spine heads in Ube3b-deficient neurons harbor increased number of NMDARs.

Our data indicate that neuronal Ube3b deficiency alters spine number and morphology. Changes of physiology of individual neurons may in turn affect development and/or activity of neuronal networks.

\subsubsection{Ube3b loss in neurons leads to imbalance between excitatory/inhibitory input to hippocampal circuits}

To test, if altered morphology and electrophysiology of neurons deleterious of Ube $3 \mathrm{~b}$ lead to defects in neuronal circuitry, we recorded kainate-induced $\gamma$-oscillatory activity in CA3 field of hippocampus. Almost all Ube3b cKO brain slices treated with $100 \mathrm{nM}$ kainate display epileptiform activity as opposed to slices from control animals (Fig. 33C). This suggests an augmented susceptibility to kainate-induced excitatory/inhibitory imbalance in hippocampal network in $U b e 3 b \mathrm{cKO}$ mice. This hypothesis is in line with an observed trend towards increased power of $\gamma$-oscillations induced by $50 \mathrm{nM}$ kainate (Fig. 33B and 33E). Interestingly, half of reported KOS patients with mutations in UBE3B are diagnosed with seizures, indicative of excitatory/inhibitory imbalance of neuronal networks.

Alteration in excitatory/inhibitory balance in $U b e 3 b \mathrm{cKO}$ neurons may be due to several factors. Intact RRP size and Pvr in Ube3b cKO neurons (Figs. 32D, and 32E) exclude malfunction of presynaptic release machinery. Given that we observed not significant increase in amplitude of evoked EPSCs (Fig. 32A), and glutamate-induced responses (Fig. $32 \mathrm{~K}$, and $32 \mathrm{~N}$ ) as well as significant increase in spine density (Fig. 31) in Ube3b cKO neurons, it is likely, that excitatory/inhibitory imbalance is attributable to augmented intrinsic excitability of $U b e 3 b$ cKO neurons.

Interestingly, spontaneous seizures in $U b e 3 b \mathrm{cKO}$ mice in the home cage were not noted. Like in the case of other mouse models of intellectual disability, e.g. Rett syndrome model, only application of kainate revealed increased susceptibility to seizures. Moreover, also in Rett syndrome mouse model, kainate treatment of brain slices revealed increased power of $\gamma$-oscillations in CA3 field of hippocampus [377]. 
Disrupted activity of neuronal networks in $U b e 3 b \mathrm{cKO}$ observed as induced seizures, and a not statistically significant increase in power of $\gamma$-oscillations may represent a primary cause of mental incapacity [378-380].

\subsubsection{Disrupted spatial memory and altered social interactions in Ube $3 b$ cKO mice}

We report that neuronal loss of Ube3b in mice disrupts spatial learning and memory and alters social interactions (Fig. 34). Interestingly, Ube $3 b \mathrm{cKO}$ mice exhibit superior social memory as compared to control animals.

Poor performance in the Morris water maze test reflecting cognitive impairments have been reported for mouse models of mental retardation syndromes, such as Down syndrome [381], $\mathrm{X}$-linked mental retardation [382], or autism spectrum disorders [383]. Behavioral defects in neuron- and glia specific-Ube3b deficient mice indicate essential roles of Ube $3 \mathrm{~b}$ in neurons and glia to control learning, memory, and social behavior.

Spatial memory impairment indicates malfunction of hippocampal CA1 region [299]. Ube3b-mediated changes of neuronal morphology concerning dendritic spines were described in the primary branches of CA1 pyramidal neurons. Our observations indicate a straightforward detrimental contribution of altered morphology of CA1 pyramidal nerve cells to circuits important for learning and memory. Possibly, morphological defects observed for pyramidal CA1 neurons reflect aberrant function of hippocampal circuitry. Interestingly, it has been reported that CA2 region of the hippocampus is essential for social memory and interaction in mice [384]. Ube3b cKO mice display a complex alterations in hippocampus-dependent behaviors. Presumptively, Ube3b cKO exerts detrimental effects on neuronal wiring in CA1 region implicated in spatial learning and improves neuronal function in CA2 subfield .

Behavior of animals and humans is associated with specific activity of neuronal networks resulting in oscillatory patterns of activity. Hippocampal $\gamma$-oscillations in mice are triggered during exploratory behavior and navigation [385-387]. Moreover, $\gamma$-oscillatory activity is correlated with behavioral performance [388]. Susceptibility of to kainate-induced seizures and altered physiology of Ube3b-deficient neurons may reflect defectful neuronal networks resulting in profound behavioral alterations in $U b e 3 b \mathrm{cKO}$ mice.

Interestingly, loss of $U b e 3 b$ in neurons and glia leads to increased sociability, which mirrors the features of behavior of some patients with intellectual disability [389]. Mentally retarded 
individuals are often characterized by 'Distanzminderung', which reflects eagerness for physical contact, and animated and happy demeanor in social interactions with foreign people [390].

Reported Ube3b-mediated loss of spatial memory and improvement in social interactions seem to be a unique feature of KOS mouse models. Down syndrome mouse model, Ts65 mice, display impairment in spatial learning, and no changes in sociability [381]. Furthermore, there are behavioral aspects of other mouse models of human diseases with intellectual disability syndromes, that were not reported for Ube3b cKO mice, such as increased aggression [391], or obsessive-compulsive-like behavior [392]. Interestingly, behavioral alterations entirely opposite to those we observe for Ube $3 b \mathrm{cKO}$ were reported for neuroligin-3 R451C mutant mouse, which exhibits augmentation of spatial learning, and impaired social interactions, characteristic features for autism spectrum disorders [393]. KOS resultant from mutations in $U B E 3 B$ represents an intellectual disability syndrome with pathology different from autism spectrum disorders. Our observations provide novel insights into the neuronal pathology of the intellectual disability.

\subsubsection{Protein targets of Ube $3 b$}

During this study, we identified several putative substrates of Ube3b by yeast two-hybrid screening, and comparative mass spectrometry (Fig. 35).

Among potential Ube3b substrates identified in yeast-two hybrid screening, G-protein coupled receptor kinase-interactor 1, Git1 regulates spine formation by Rac1-downstream effectors $\alpha$-p21-activated kinases, PAKs. Active PAKs promote formation of dendritic protrusions, which correlates with number of excitatory synapses [394]. However, Ube3bmediated Git1 ubiquitylation, and if Git1 overexpression might be attributable to Ube3bmediated increase of spines should be validated in the future.

Additionally, we report up-regulation of gamma isoform of protein phosphatase 3 catalytic subunit, Ppp3cc in SM3 fractions of Ube3b cKO brain. Ppp3cc is a part of large protein phosphatase complex, also known as calcineurin. Its role in neurons remains to be thoroughly studied, however it has been described in dendritic spines [395], where it localizes to F-actin, and regulates cytoskeleton stability [396]. Those features make Ppp3cc a promising Ube3b target, underlying the molecular machinery of intellectual disability in KOS patients. 


\section{REFERENCES}

[1] Gaier, E.D., Rodriguiz, R.M., Ma, X.M., Sivaramakrishnan, S., Bousquet-Moore, D., Wetsel, W.C., Eipper, B.A. and Mains, R.E. (2010). Haploinsufficiency in peptidylglycine alpha-amidating monooxygenase leads to altered synaptic transmission in the amygdala and impaired emotional responses. J Neurosci 30, 13656-69.

[2] Zeilhofer, H.U., Wildner, H. and Yevenes, G.E. (2012). Fast synaptic inhibition in spinal sensory processing and pain control. Physiol Rev 92, 193-235.

[3] Lange, W. (1975). Cell number and cell density in the cerebellar cortex of man and some other mammals. Cell Tissue Res 157, 115-24.

[4] Shariff, G.A. (1953). Cell counts in the primate cerebral cortex. J Comp Neurol 98, 381-400.

[5] Kawabe, H. and Brose, N. (2011). The role of ubiquitylation in nerve cell development. Nat Rev Neurosci 12, 251-68.

[6] Rakic, P. (1995). A small step for the cell, a giant leap for mankind: a hypothesis of neocortical expansion during evolution. Trends Neurosci 18, 383-8.

[7] McConnell, S.K. (1995). Constructing the cerebral cortex: neurogenesis and fate determination. Neuron 15, 761-8.

[8] Taverna, E., Gotz, M. and Huttner, W.B. (2014). The cell biology of neurogenesis: toward an understanding of the development and evolution of the neocortex. Annu Rev Cell Dev Biol 30, 465-502.

[9] Huttner, W.B. and Brand, M. (1997). Asymmetric division and polarity of neuroepithelial cells. Curr Opin Neurobiol 7, 29-39.

[10] Wodarz, A. and Huttner, W.B. (2003). Asymmetric cell division during neurogenesis in Drosophila and vertebrates. Mech Dev 120, 1297-309.

[11] Aaku-Saraste, E., Hellwig, A. and Huttner, W.B. (1996). Loss of occludin and functional tight junctions, but not $\mathrm{ZO}-1$, during neural tube closure--remodeling of the neuroepithelium prior to neurogenesis. Dev Biol 180, 664-79.

[12] Aaku-Saraste, E., Oback, B., Hellwig, A. and Huttner, W.B. (1997). Neuroepithelial cells downregulate their plasma membrane polarity prior to neural tube closure and neurogenesis. Mech Dev 69, 71-81.

[13] Malatesta, P., Hartfuss, E. and Gotz, M. (2000). Isolation of radial glial cells by fluorescent-activated cell sorting reveals a neuronal lineage. Development 127, 5253-63.

[14] Hartfuss, E., Galli, R., Heins, N. and Gotz, M. (2001). Characterization of CNS precursor subtypes and radial glia. Dev Biol 229, 15-30.

[15] Chenn, A., Zhang, Y.A., Chang, B.T. and McConnell, S.K. (1998). Intrinsic polarity of mammalian neuroepithelial cells. Mol Cell Neurosci 11, 183-93.

[16] Malatesta, P., Hack, M.A., Hartfuss, E., Kettenmann, H., Klinkert, W., Kirchhoff, F. and Gotz, M. (2003). Neuronal or glial progeny: regional differences in radial glia fate. Neuron 37, 751-64.

[17] Weigmann, A., Corbeil, D., Hellwig, A. and Huttner, W.B. (1997). Prominin, a novel microvilli-specific polytopic membrane protein of the apical surface of epithelial cells, is targeted to plasmalemmal protrusions of non-epithelial cells. Proc Natl Acad Sci U S A 94, 12425-30.

[18] Halfter, W., Dong, S., Yip, Y.P., Willem, M. and Mayer, U. (2002). A critical function of the pial basement membrane in cortical histogenesis. J Neurosci 22, 6029-40. 
[19] Pilz, G.A. et al. (2013). Amplification of progenitors in the mammalian telencephalon includes a new radial glial cell type. Nat Commun 4, 2125.

[20] Patten, B.A., Sardi, S.P., Koirala, S., Nakafuku, M. and Corfas, G. (2006). Notch1 signaling regulates radial glia differentiation through multiple transcriptional mechanisms. J Neurosci 26, 3102-8.

[21] Schmid, R.S., McGrath, B., Berechid, B.E., Boyles, B., Marchionni, M., Sestan, N. and Anton, E.S. (2003). Neuregulin 1-erbB2 signaling is required for the establishment of radial glia and their transformation into astrocytes in cerebral cortex. Proc Natl Acad Sci U S A 100, 4251-6.

[22] Lavado, A. and Oliver, G. (2011). Six3 is required for ependymal cell maturation. Development 138, 5291-300.

[23] Englund, C., Fink, A., Lau, C., Pham, D., Daza, R.A., Bulfone, A., Kowalczyk, T. and Hevner, R.F. (2005). Pax6, Tbr2, and Tbr1 are expressed sequentially by radial glia, intermediate progenitor cells, and postmitotic neurons in developing neocortex. J Neurosci 25, 247-51.

[24] Heins, N. et al. (2002). Glial cells generate neurons: the role of the transcription factor Pax6. Nat Neurosci 5, 308-15.

[25] Gotz, M., Stoykova, A. and Gruss, P. (1998). Pax6 controls radial glia differentiation in the cerebral cortex. Neuron 21, 1031-44.

[26] Miyata, T., Kawaguchi, A., Saito, K., Kawano, M., Muto, T. and Ogawa, M. (2004). Asymmetric production of surface-dividing and non-surface-dividing cortical progenitor cells. Development 131, 3133-45.

[27] Noctor, S.C., Martinez-Cerdeno, V., Ivic, L. and Kriegstein, A.R. (2004). Cortical neurons arise in symmetric and asymmetric division zones and migrate through specific phases. Nat Neurosci 7, 136-44.

[28] Tarabykin, V., Stoykova, A., Usman, N. and Gruss, P. (2001). Cortical upper layer neurons derive from the subventricular zone as indicated by Svet1 gene expression. Development 128, 1983-93.

[29] Nieto, M. et al. (2004). Expression of Cux-1 and Cux-2 in the subventricular zone and upper layers II-IV of the cerebral cortex. J Comp Neurol 479, 168-80.

[30] Haubensak, W., Attardo, A., Denk, W. and Huttner, W.B. (2004). Neurons arise in the basal neuroepithelium of the early mammalian telencephalon: a major site of neurogenesis. Proc Natl Acad Sci U S A 101, 3196-201.

[31] Anderson, S.A., Marin, O., Horn, C., Jennings, K. and Rubenstein, J.L. (2001). Distinct cortical migrations from the medial and lateral ganglionic eminences. Development 128, 353-63.

[32] Anton, E.S., Cameron, R.S. and Rakic, P. (1996). Role of neuron-glial junctional domain proteins in the maintenance and termination of neuronal migration across the embryonic cerebral wall. J Neurosci 16, 2283-93.

[33] Rakic, P., Cameron, R.S. and Komuro, H. (1994). Recognition, adhesion, transmembrane signaling and cell motility in guided neuronal migration. Curr Opin Neurobiol 4, 63-9.

[34] Polleux, F. and Snider, W. (2010). Initiating and growing an axon. Cold Spring Harb Perspect Biol 2, a001925.

[35] Gleeson, J.G., Lin, P.T., Flanagan, L.A. and Walsh, C.A. (1999). Doublecortin is a microtubule-associated protein and is expressed widely by migrating neurons. Neuron 23, 257-71.

[36] Gleeson, J.G. et al. (1998). Doublecortin, a brain-specific gene mutated in human Xlinked lissencephaly and double cortex syndrome, encodes a putative signaling protein. Cell 92, 63-72. 
[37] Taylor, K.R., Holzer, A.K., Bazan, J.F., Walsh, C.A. and Gleeson, J.G. (2000). Patient mutations in doublecortin define a repeated tubulin-binding domain. J Biol Chem 275, 34442-50.

[38] Reiner, O., Carrozzo, R., Shen, Y., Wehnert, M., Faustinella, F., Dobyns, W.B., Caskey, C.T. and Ledbetter, D.H. (1993). Isolation of a Miller-Dieker lissencephaly gene containing $\mathrm{G}$ protein beta-subunit-like repeats. Nature 364, 717-21.

[39] Cahana, A. et al. (2001). Targeted mutagenesis of Lis 1 disrupts cortical development and LIS1 homodimerization. Proc Natl Acad Sci U S A 98, 6429-34.

[40] Caviness, V.S., Jr., So, D.K. and Sidman, R.L. (1972). The hybrid reeler mouse. J Hered 63, 241-6.

[41] Ogawa, M., Miyata, T., Nakajima, K., Yagyu, K., Seike, M., Ikenaka, K., Yamamoto, H. and Mikoshiba, K. (1995). The reeler gene-associated antigen on Cajal-Retzius neurons is a crucial molecule for laminar organization of cortical neurons. Neuron 14, 899-912.

[42] D'Arcangelo, G., Miao, G.G., Chen, S.C., Soares, H.D., Morgan, J.I. and Curran, T. (1995). A protein related to extracellular matrix proteins deleted in the mouse mutant reeler. Nature 374, 719-23.

[43] Gonzalez, J.L., Russo, C.J., Goldowitz, D., Sweet, H.O., Davisson, M.T. and Walsh, C.A. (1997). Birthdate and cell marker analysis of scrambler: a novel mutation affecting cortical development with a reeler-like phenotype. J Neurosci 17, 9204-11.

[44] Trommsdorff, M. et al. (1999). Reeler/Disabled-like disruption of neuronal migration in knockout mice lacking the VLDL receptor and ApoE receptor 2. Cell 97, 689-701.

[45] D'Arcangelo, G., Homayouni, R., Keshvara, L., Rice, D.S., Sheldon, M. and Curran, T. (1999). Reelin is a ligand for lipoprotein receptors. Neuron 24, 471-9.

[46] Koleske, A.J. (2013). Molecular mechanisms of dendrite stability. Nat Rev Neurosci $14,536-50$.

[47] Shelly, M. and Poo, M.M. (2011). Role of LKB1-SAD/MARK pathway in neuronal polarization. Dev Neurobiol 71, 508-27.

[48] Bradke, F. and Dotti, C.G. (1997). Neuronal polarity: vectorial cytoplasmic flow precedes axon formation. Neuron 19, 1175-86.

[49] Zheng, Y. et al. (2008). Dynein is required for polarized dendritic transport and uniform microtubule orientation in axons. Nat Cell Biol 10, 1172-80.

[50] Ahmad, F.J., Echeverri, C.J., Vallee, R.B. and Baas, P.W. (1998). Cytoplasmic dynein and dynactin are required for the transport of microtubules into the axon. $J$ Cell Biol 140, 391-401.

[51] Harada, A. et al. (1994). Altered microtubule organization in small-calibre axons of mice lacking tau protein. Nature 369, 488-91.

[52] Dawson, H.N., Ferreira, A., Eyster, M.V., Ghoshal, N., Binder, L.I. and Vitek, M.P. (2001). Inhibition of neuronal maturation in primary hippocampal neurons from tau deficient mice. J Cell Sci 114, 1179-87.

[53] Hirokawa, N., Niwa, S. and Tanaka, Y. (2010). Molecular motors in neurons: transport mechanisms and roles in brain function, development, and disease. Neuron 68, 610-38.

[54] Song, A.H., Wang, D., Chen, G., Li, Y., Luo, J., Duan, S. and Poo, M.M. (2009). A selective filter for cytoplasmic transport at the axon initial segment. Cell 136, 114860 .

[55] Galiano, M.R. et al. (2012). A distal axonal cytoskeleton forms an intra-axonal boundary that controls axon initial segment assembly. Cell 149, 1125-39. 
[56] Witte, H., Neukirchen, D. and Bradke, F. (2008). Microtubule stabilization specifies initial neuronal polarization. J Cell Biol 180, 619-32.

[57] Bradke, F. and Dotti, C.G. (1999). The role of local actin instability in axon formation. Science 283, 1931-4.

[58] Okamoto, K., Nagai, T., Miyawaki, A. and Hayashi, Y. (2004). Rapid and persistent modulation of actin dynamics regulates postsynaptic reorganization underlying bidirectional plasticity. Nat Neurosci 7, 1104-12.

[59] Matsuzaki, M., Honkura, N., Ellis-Davies, G.C. and Kasai, H. (2004). Structural basis of long-term potentiation in single dendritic spines. Nature 429, 761-6.

[60] Zhou, Q., Homma, K.J. and Poo, M.M. (2004). Shrinkage of dendritic spines associated with long-term depression of hippocampal synapses. Neuron 44, 749-57.

[61] Kim, I.H., Racz, B., Wang, H., Burianek, L., Weinberg, R., Yasuda, R., Wetsel, W.C. and Soderling, S.H. (2013). Disruption of Arp2/3 results in asymmetric structural plasticity of dendritic spines and progressive synaptic and behavioral abnormalities. J Neurosci 33, 6081-92.

[62] Racz, B. and Weinberg, R.J. (2008). Organization of the Arp2/3 complex in hippocampal spines. J Neurosci 28, 5654-9.

[63] Bai, J., Ramos, R.L., Paramasivam, M., Siddiqi, F., Ackman, J.B. and LoTurco, J.J. (2008). The role of DCX and LIS1 in migration through the lateral cortical stream of developing forebrain. Dev Neurosci 30, 144-56.

[64] Nakahira, E. and Yuasa, S. (2005). Neuronal generation, migration, and differentiation in the mouse hippocampal primoridium as revealed by enhanced green fluorescent protein gene transfer by means of in utero electroporation. J Comp Neurol 483, 329-40.

[65] Remedios, R. et al. (2007). A stream of cells migrating from the caudal telencephalon reveals a link between the amygdala and neocortex. Nat Neurosci 10, 1141-50.

[66] Tabata, H. and Nakajima, K. (2001). Efficient in utero gene transfer system to the developing mouse brain using electroporation: visualization of neuronal migration in the developing cortex. Neuroscience 103, 865-72.

[67] Borrell, V., Yoshimura, Y. and Callaway, E.M. (2005). Targeted gene delivery to telencephalic inhibitory neurons by directional in utero electroporation. J Neurosci Methods 143, 151-8.

[68] Punzo, C. and Cepko, C.L. (2008). Ultrasound-guided in utero injections allow studies of the development and function of the eye. Dev Dyn 237, 1034-42.

[69] Niwa, M. et al. (2010). Knockdown of DISC1 by in utero gene transfer disturbs postnatal dopaminergic maturation in the frontal cortex and leads to adult behavioral deficits. Neuron 65, 480-9.

[70] Manent, J.B., Wang, Y., Chang, Y., Paramasivam, M. and LoTurco, J.J. (2009). Dex reexpression reduces subcortical band heterotopia and seizure threshold in an animal model of neuronal migration disorder. Nat Med 15, 84-90.

[71] LoTurco, J., Manent, J.B. and Sidiqi, F. (2009). New and improved tools for in utero electroporation studies of developing cerebral cortex. Cereb Cortex 19 Suppl 1, i1205.

[72] dal Maschio, M. et al. (2012). High-performance and site-directed in utero electroporation by a triple-electrode probe. Nat Commun 3, 960.

[73] Kolk, S.M., de Mooij-Malsen, A.J. and Martens, G.J. (2011). Spatiotemporal Molecular Approach of in utero Electroporation to Functionally Decipher Endophenotypes in Neurodevelopmental Disorders. Front Mol Neurosci 4, 37.

[74] Ciechanover, A., Elias, S., Heller, H. and Hershko, A. (1982). "Covalent affinity" purification of ubiquitin-activating enzyme. J Biol Chem 257, 2537-42. 
[75] Hershko, A., Heller, H., Elias, S. and Ciechanover, A. (1983). Components of ubiquitin-protein ligase system. Resolution, affinity purification, and role in protein breakdown. J Biol Chem 258, 8206-14.

[76] Hershko, A., Leshinsky, E., Ganoth, D. and Heller, H. (1984). ATP-dependent degradation of ubiquitin-protein conjugates. Proc Natl Acad Sci U S A 81, 1619-23.

[77] Baumeister, W., Walz, J., Zuhl, F. and Seemuller, E. (1998). The proteasome: paradigm of a self-compartmentalizing protease. Cell 92, 367-80.

[78] Pickart, C.M. and Cohen, R.E. (2004). Proteasomes and their kin: proteases in the machine age. Nat Rev Mol Cell Biol 5, 177-87.

[79] Rechsteiner, M. and Hill, C.P. (2005). Mobilizing the proteolytic machine: cell biological roles of proteasome activators and inhibitors. Trends Cell Biol 15, 27-33.

[80] Amerik, A.Y. and Hochstrasser, M. (2004). Mechanism and function of deubiquitinating enzymes. Biochim Biophys Acta 1695, 189-207.

[81] Ciechanover, A., Finley, D. and Varshavsky, A. (1984). Ubiquitin dependence of selective protein degradation demonstrated in the mammalian cell cycle mutant ts 85 . Cell 37, 57-66.

[82] Chau, V., Tobias, J.W., Bachmair, A., Marriott, D., Ecker, D.J., Gonda, D.K. and Varshavsky, A. (1989). A multiubiquitin chain is confined to specific lysine in a targeted short-lived protein. Science 243, 1576-83.

[83] Xu, P. et al. (2009). Quantitative proteomics reveals the function of unconventional ubiquitin chains in proteasomal degradation. Cell 137, 133-45.

[84] Ramaekers, C.H., van den Beucken, T., Bristow, R.G., Chiu, R.K., Durocher, D. and Wouters, B.G. (2014). RNF8-independent Lys63 poly-ubiquitylation prevents genomic instability in response to replication-associated DNA damage. PLoS One 9, e89997.

[85] Goh, L.K. and Sorkin, A. (2013). Endocytosis of receptor tyrosine kinases. Cold Spring Harb Perspect Biol 5, a017459.

[86] Hicke, L. and Riezman, H. (1996). Ubiquitination of a yeast plasma membrane receptor signals its ligand-stimulated endocytosis. Cell 84, 277-87.

[87] Williamson, A., Wickliffe, K.E., Mellone, B.G., Song, L., Karpen, G.H. and Rape, M. (2009). Identification of a physiological E2 module for the human anaphasepromoting complex. Proc Natl Acad Sci U S A 106, 18213-8.

[88] Locke, M., Toth, J.I. and Petroski, M.D. (2014). Lys11- and Lys48-linked ubiquitin chains interact with p97 during endoplasmic-reticulum-associated degradation. Biochem J 459, 205-16.

[89] Huibregtse, J.M., Scheffner, M., Beaudenon, S. and Howley, P.M. (1995). A family of proteins structurally and functionally related to the E6-AP ubiquitin-protein ligase. Proc Natl Acad Sci U S A 92, 2563-7.

[90] Barlow, P.N., Luisi, B., Milner, A., Elliott, M. and Everett, R. (1994). Structure of the $\mathrm{C} 3 \mathrm{HC} 4$ domain by $1 \mathrm{H}$-nuclear magnetic resonance spectroscopy. A new structural class of zinc-finger. J Mol Biol 237, 201-11.

[91] Maspero, E., Mari, S., Valentini, E., Musacchio, A., Fish, A., Pasqualato, S. and Polo, S. (2011). Structure of the HECT:ubiquitin complex and its role in ubiquitin chain elongation. EMBO Rep 12, 342-9.

[92] Rotin, D. and Kumar, S. (2009). Physiological functions of the HECT family of ubiquitin ligases. Nat Rev Mol Cell Biol 10, 398-409.

[93] Kumar, S., Harvey, K.F., Kinoshita, M., Copeland, N.G., Noda, M. and Jenkins, N.A. (1997). cDNA cloning, expression analysis, and mapping of the mouse Nedd4 gene. Genomics 40, 435-43. 
[94] Hochrainer, K., Mayer, H., Baranyi, U., Binder, B., Lipp, J. and Kroismayr, R. (2005). The human HERC family of ubiquitin ligases: novel members, genomic organization, expression profiling, and evolutionary aspects. Genomics 85, 153-64.

[95] Scott, A.M., Antal, C.E. and Newton, A.C. (2013). Electrostatic and hydrophobic interactions differentially tune membrane binding kinetics of the $\mathrm{C} 2$ domain of protein kinase Calpha. J Biol Chem 288, 16905-15.

[96] Cheng, P.L., Lu, H., Shelly, M., Gao, H. and Poo, M.M. (2011). Phosphorylation of E3 ligase Smurf1 switches its substrate preference in support of axon development. Neuron 69, 231-43.

[97] Kawabe, H. et al. (2010). Regulation of Rap2A by the ubiquitin ligase Nedd4-1 controls neurite development. Neuron 65, 358-72.

[98] Hsia, H.E. et al. (2014). Ubiquitin E3 ligase Nedd4-1 acts as a downstream target of PI3K/PTEN-mTORC1 signaling to promote neurite growth. Proc Natl Acad Sci U S A $111,13205-10$.

[99] Scudder, S.L., Goo, M.S., Cartier, A.E., Molteni, A., Schwarz, L.A., Wright, R. and Patrick, G.N. (2014). Synaptic strength is bidirectionally controlled by opposing activity-dependent regulation of Nedd4-1 and USP8. J Neurosci 34, 16637-49.

[100] Widagdo, J., Chai, Y.J., Ridder, M.C., Chau, Y.Q., Johnson, R.C., Sah, P., Huganir, R.L. and Anggono, V. (2015). Activity-Dependent Ubiquitination of GluA1 and GluA2 Regulates AMPA Receptor Intracellular Sorting and Degradation. Cell Rep

[101] Yuen, E.Y., Wei, J., Liu, W., Zhong, P., Li, X. and Yan, Z. (2012). Repeated stress causes cognitive impairment by suppressing glutamate receptor expression and function in prefrontal cortex. Neuron 73, 962-77.

[102] Schwarz, L.A., Hall, B.J. and Patrick, G.N. (2010). Activity-dependent ubiquitination of GluA1 mediates a distinct AMPA receptor endocytosis and sorting pathway. J Neurosci 30, 16718-29.

[103] Andersen, M.N., Krzystanek, K., Jespersen, T., Olesen, S.P. and Rasmussen, H.B. (2012). AMP-activated protein kinase downregulates Kv7.1 cell surface expression. Traffic 13, 143-56.

[104] Rougier, J.S. et al. (2005). Molecular determinants of voltage-gated sodium channel regulation by the Nedd4/Nedd4-like proteins. Am J Physiol Cell Physiol 288, C692701.

[105] Ekberg, J.A., Boase, N.A., Rychkov, G., Manning, J., Poronnik, P. and Kumar, S. (2014). Nedd4-2 (NEDD4L) controls intracellular $\mathrm{Na}(+)$-mediated activity of voltage-gated sodium channels in primary cortical neurons. Biochem J 457, 27-31.

[106] Cachemaille, M., Laedermann, C.J., Pertin, M., Abriel, H., Gosselin, R.D. and Decosterd, I. (2012). Neuronal expression of the ubiquitin ligase Nedd4-2 in rat dorsal root ganglia: modulation in the spared nerve injury model of neuropathic pain. Neuroscience 227, 370-80.

[107] Laedermann, C.J. et al. (2013). Dysregulation of voltage-gated sodium channels by ubiquitin ligase NEDD4-2 in neuropathic pain. J Clin Invest 123, 3002-13.

[108] Sorkina, T., Miranda, M., Dionne, K.R., Hoover, B.R., Zahniser, N.R. and Sorkin, A. (2006). RNA interference screen reveals an essential role of Nedd4-2 in dopamine transporter ubiquitination and endocytosis. J Neurosci 26, 8195-205.

[109] Vina-Vilaseca, A. and Sorkin, A. (2010). Lysine 63-linked polyubiquitination of the dopamine transporter requires WW3 and WW4 domains of Nedd4-2 and UBE2D ubiquitin-conjugating enzymes. J Biol Chem 285, 7645-56.

[110] Boehmer, C., Palmada, M., Rajamanickam, J., Schniepp, R., Amara, S. and Lang, F. (2006). Post-translational regulation of EAAT2 function by co-expressed ubiquitin ligase Nedd4-2 is impacted by SGK kinases. J Neurochem 97, 911-21. 
[111] Rougeulle, C., Glatt, H. and Lalande, M. (1997). The Angelman syndrome candidate gene, UBE3A/E6-AP, is imprinted in brain. Nat Genet 17, 14-5.

[112] Chamberlain, S.J. and Brannan, C.I. (2001). The Prader-Willi syndrome imprinting center activates the paternally expressed murine Ube3a antisense transcript but represses paternal Ube3a. Genomics 73, 316-22.

[113] Steffenburg, S., Gillberg, C.L., Steffenburg, U. and Kyllerman, M. (1996). Autism in Angelman syndrome: a population-based study. Pediatr Neurol 14, 131-6.

[114] Malcolm, S., Clayton-Smith, J., Nichols, M., Robb, S., Webb, T., Armour, J.A., Jeffreys, A.J. and Pembrey, M.E. (1991). Uniparental paternal disomy in Angelman's syndrome. Lancet 337, 694-7.

[115] Knoll, J.H., Nicholls, R.D., Magenis, R.E., Graham, J.M., Jr., Lalande, M. and Latt, S.A. (1989). Angelman and Prader-Willi syndromes share a common chromosome 15 deletion but differ in parental origin of the deletion. Am J Med Genet 32, 285-90.

[116] Kishino, T., Lalande, M. and Wagstaff, J. (1997). UBE3A/E6-AP mutations cause Angelman syndrome. Nat Genet 15, 70-3.

[117] Jiang, Y.H., Armstrong, D., Albrecht, U., Atkins, C.M., Noebels, J.L., Eichele, G., Sweatt, J.D. and Beaudet, A.L. (1998). Mutation of the Angelman ubiquitin ligase in mice causes increased cytoplasmic p53 and deficits of contextual learning and longterm potentiation. Neuron 21, 799-811.

[118] Miura, K., Kishino, T., Li, E., Webber, H., Dikkes, P., Holmes, G.L. and Wagstaff, J. (2002). Neurobehavioral and electroencephalographic abnormalities in Ube3a maternal-deficient mice. Neurobiol Dis 9, 149-59.

[119] Weeber, E.J. et al. (2003). Derangements of hippocampal calcium/calmodulindependent protein kinase II in a mouse model for Angelman mental retardation syndrome. J Neurosci 23, 2634-44.

[120] Miao, S., Chen, R., Ye, J., Tan, G.H., Li, S., Zhang, J., Jiang, Y.H. and Xiong, Z.Q. (2013). The Angelman syndrome protein Ube3a is required for polarized dendrite morphogenesis in pyramidal neurons. J Neurosci 33, 327-33.

[121] Su, H. et al. (2011). Mitochondrial dysfunction in CA1 hippocampal neurons of the UBE3A deficient mouse model for Angelman syndrome. Neurosci Lett 487, 129-33.

[122] Yashiro, K. et al. (2009). Ube3a is required for experience-dependent maturation of the neocortex. Nat Neurosci 12, 777-83.

[123] Greer, P.L. et al. (2010). The Angelman Syndrome protein Ube3A regulates synapse development by ubiquitinating arc. Cell 140, 704-16.

[124] Kuhnle, S., Mothes, B., Matentzoglu, K. and Scheffner, M. (2013). Role of the ubiquitin ligase E6AP/UBE3A in controlling levels of the synaptic protein Arc. Proc Natl Acad Sci U S A 110, 8888-93.

[125] Margolis, S.S. et al. (2010). EphB-mediated degradation of the RhoA GEF Ephexin5 relieves a developmental brake on excitatory synapse formation. Cell 143, 442-55.

[126] Yi, J.J., Berrios, J., Newbern, J.M., Snider, W.D., Philpot, B.D., Hahn, K.M. and Zylka, M.J. (2015). An Autism-Linked Mutation Disables Phosphorylation Control of UBE3A. Cell 162, 795-807.

[127] Hoeller, D., Hecker, C.M. and Dikic, I. (2006). Ubiquitin and ubiquitin-like proteins in cancer pathogenesis. Nat Rev Cancer 6, 776-88.

[128] Lohr, N.J. et al. (2010). Human ITCH E3 ubiquitin ligase deficiency causes syndromic multisystem autoimmune disease. Am J Hum Genet 86, 447-53.

[129] Ambrozkiewicz, M.C. and Kawabe, H. (2015). HECT-type E3 ubiquitin ligases in nerve cell development and synapse physiology. FEBS Lett 589, 1635-43. 
[130] Brooks, S.S., Wisniewski, K. and Brown, W.T. (1994). New X-linked mental retardation (XLMR) syndrome with distinct facial appearance and growth retardation. Am J Med Genet 51, 586-90.

[131] Froyen, G. et al. (2008). Submicroscopic duplications of the hydroxysteroid dehydrogenase HSD17B10 and the E3 ubiquitin ligase HUWE1 are associated with mental retardation. Am J Hum Genet 82, 432-43.

[132] Morava, E., Storcz, J. and Kosztolanyi, G. (1996). X-linked mental retardation syndrome: three brothers with the Brooks-Wisniewski-Brown syndrome. Am J Med Genet 64, 59-62.

[133] Clayton-Smith, J. and Laan, L. (2003). Angelman syndrome: a review of the clinical and genetic aspects. J Med Genet 40, 87-95.

[134] O'Roak, B.J. et al. (2012). Sporadic autism exomes reveal a highly interconnected protein network of de novo mutations. Nature 485, 246-50.

[135] Bayes, A., Collins, M.O., Croning, M.D., van de Lagemaat, L.N., Choudhary, J.S. and Grant, S.G. (2012). Comparative study of human and mouse postsynaptic proteomes finds high compositional conservation and abundance differences for key synaptic proteins. PLoS One 7, e46683.

[136] Basel-Vanagaite, L. et al. (2012). Deficiency for the ubiquitin ligase UBE3B in a blepharophimosis-ptosis-intellectual-disability syndrome. Am J Hum Genet 91, 9981010.

[137] Basel-Vanagaite, L. et al. (2014). Expanding the clinical and mutational spectrum of Kaufman oculocerebrofacial syndrome with biallelic UBE3B mutations. Hum Genet 133, 939-49.

[138] Flex, E. et al. (2013). Loss of function of the E3 ubiquitin-protein ligase UBE3B causes Kaufman oculocerebrofacial syndrome. J Med Genet 50, 493-9.

[139] Pedurupillay, C.R. et al. (2015). Kaufman oculocerebrofacial syndrome in sisters with novel compound heterozygous mutation in UBE3B. Am J Med Genet A 167, 657-63.

[140] Kaufman, R.L., Rimoin, D.L., Prensky, A.L. and Sly, W.S. (1971). An oculocerebrofacial syndrome. Birth Defects Orig Artic Ser 7, 135-8.

[141] Gong, T.W., Hegeman, A.D., Shin, J.J., Adler, H.J., Raphael, Y. and Lomax, M.I. (1996). Identification of genes expressed after noise exposure in the chick basilar papilla. Hear Res 96, 20-32.

[142] Gong, T.W., Huang, L., Warner, S.J. and Lomax, M.I. (2003). Characterization of the human UBE3B gene: structure, expression, evolution, and alternative splicing. Genomics 82, 143-52.

[143] Chahrour, M.H. et al. (2012). Whole-exome sequencing and homozygosity analysis implicate depolarization-regulated neuronal genes in autism. PLoS Genet 8, e1002635.

[144] Lee, R.C., Feinbaum, R.L. and Ambros, V. (1993). The C. elegans heterochronic gene lin-4 encodes small RNAs with antisense complementarity to lin-14. Cell 75, 843-54.

[145] Wightman, B., Ha, I. and Ruvkun, G. (1993). Posttranscriptional regulation of the heterochronic gene lin-14 by lin- 4 mediates temporal pattern formation in C. elegans. Cell 75, 855-62.

[146] Friedman, R.C., Farh, K.K., Burge, C.B. and Bartel, D.P. (2009). Most mammalian mRNAs are conserved targets of microRNAs. Genome Res 19, 92-105.

[147] Lee, Y. et al. (2003). The nuclear RNase III Drosha initiates microRNA processing. Nature 425, 415-9. 
[148] Han, J., Lee, Y., Yeom, K.H., Kim, Y.K., Jin, H. and Kim, V.N. (2004). The DroshaDGCR8 complex in primary microRNA processing. Genes Dev 18, 3016-27.

[149] Blaszczyk, J., Tropea, J.E., Bubunenko, M., Routzahn, K.M., Waugh, D.S., Court, D.L. and Ji, X. (2001). Crystallographic and modeling studies of RNase III suggest a mechanism for double-stranded RNA cleavage. Structure 9, 1225-36.

[150] Zhang, H., Kolb, F.A., Jaskiewicz, L., Westhof, E. and Filipowicz, W. (2004). Single processing center models for human Dicer and bacterial RNase III. Cell 118, 57-68.

[151] Guil, S. and Caceres, J.F. (2007). The multifunctional RNA-binding protein hnRNP A1 is required for processing of miR-18a. Nat Struct Mol Biol 14, 591-6.

[152] Trabucchi, M., Briata, P., Garcia-Mayoral, M., Haase, A.D., Filipowicz, W., Ramos, A., Gherzi, R. and Rosenfeld, M.G. (2009). The RNA-binding protein KSRP promotes the biogenesis of a subset of microRNAs. Nature 459, 1010-4.

[153] Lund, E., Guttinger, S., Calado, A., Dahlberg, J.E. and Kutay, U. (2004). Nuclear export of microRNA precursors. Science 303, 95-8.

[154] Xie, M., Li, M., Vilborg, A., Lee, N., Shu, M.D., Yartseva, V., Sestan, N. and Steitz, J.A. (2013). Mammalian 5'-capped microRNA precursors that generate a single microRNA. Cell 155, 1568-80.

[155] MacRae, I.J., Zhou, K. and Doudna, J.A. (2007). Structural determinants of RNA recognition and cleavage by Dicer. Nat Struct Mol Biol 14, 934-40.

[156] Meister, G., Landthaler, M., Patkaniowska, A., Dorsett, Y., Teng, G. and Tuschl, T. (2004). Human Argonaute 2 mediates RNA cleavage targeted by miRNAs and siRNAs. Mol Cell 15, 185-97.

[157] Dueck, A., Ziegler, C., Eichner, A., Berezikov, E. and Meister, G. (2012). microRNAs associated with the different human Argonaute proteins. Nucleic Acids Res 40, 9850-62.

[158] Yoda, M., Kawamata, T., Paroo, Z., Ye, X., Iwasaki, S., Liu, Q. and Tomari, Y. (2010). ATP-dependent human RISC assembly pathways. Nat Struct Mol Biol 17, 17-23.

[159] Iwasaki, S., Kobayashi, M., Yoda, M., Sakaguchi, Y., Katsuma, S., Suzuki, T. and Tomari, Y. (2010). Hsc70/Hsp90 chaperone machinery mediates ATP-dependent RISC loading of small RNA duplexes. Mol Cell 39, 292-9.

[160] Kawamata, T., Seitz, H. and Tomari, Y. (2009). Structural determinants of miRNAs for RISC loading and slicer-independent unwinding. Nat Struct Mol Biol 16, $953-$ 60.

[161] Nykanen, A., Haley, B. and Zamore, P.D. (2001). ATP requirements and small interfering RNA structure in the RNA interference pathway. Cell 107, 309-21.

[162] Khvorova, A., Reynolds, A. and Jayasena, S.D. (2003). Functional siRNAs and miRNAs exhibit strand bias. Cell 115, 209-16.

[163] Wu, H., Ye, C., Ramirez, D. and Manjunath, N. (2009). Alternative processing of primary microRNA transcripts by Drosha generates 5 ' end variation of mature microRNA. PLoS One 4, e7566.

[164] Krol, J., Loedige, I. and Filipowicz, W. (2010). The widespread regulation of microRNA biogenesis, function and decay. Nat Rev Genet 11, 597-610.

[165] Ha, M. and Kim, V.N. (2014). Regulation of microRNA biogenesis. Nat Rev Mol Cell Biol 15, 509-24.

[166] Vasudevan, S. and Steitz, J.A. (2007). AU-rich-element-mediated upregulation of translation by FXR1 and Argonaute 2. Cell 128, 1105-18.

[167] Yamashita, A., Chang, T.C., Yamashita, Y., Zhu, W., Zhong, Z., Chen, C.Y. and Shyu, A.B. (2005). Concerted action of poly(A) nucleases and decapping enzyme in mammalian mRNA turnover. Nat Struct Mol Biol 12, 1054-63. 
[168] Iwasaki, S., Kawamata, T. and Tomari, Y. (2009). Drosophila argonaute1 and argonaute2 employ distinct mechanisms for translational repression. Mol Cell 34, 58-67.

[169] Fabian, M.R. et al. (2009). Mammalian miRNA RISC recruits CAF1 and PABP to affect PABP-dependent deadenylation. Mol Cell 35, 868-80.

[170] Chendrimada, T.P., Finn, K.J., Ji, X., Baillat, D., Gregory, R.I., Liebhaber, S.A., Pasquinelli, A.E. and Shiekhattar, R. (2007). MicroRNA silencing through RISC recruitment of eIF6. Nature 447, 823-8.

[171] Ding, X.C., Slack, F.J. and Grosshans, H. (2008). The let-7 microRNA interfaces extensively with the translation machinery to regulate cell differentiation. Cell Cycle 7, 3083-90.

[172] Yang, J. et al. (2011). MiR-140 is co-expressed with Wwp2-C transcript and activated by Sox 9 to target Sp1 in maintaining the chondrocyte proliferation. FEBS Lett 585, 2992-7.

[173] O'Carroll, D. and Schaefer, A. (2013). General principals of miRNA biogenesis and regulation in the brain. Neuropsychopharmacology 38, 39-54.

[174] Li, Z. and Rana, T.M. (2014). Therapeutic targeting of microRNAs: current status and future challenges. Nat Rev Drug Discov 13, 622-38.

[175] Kawase-Koga, Y., Otaegi, G. and Sun, T. (2009). Different timings of Dicer deletion affect neurogenesis and gliogenesis in the developing mouse central nervous system. Dev Dyn 238, 2800-12.

[176] Volvert, M.L. et al. (2014). MicroRNA targeting of CoREST controls polarization of migrating cortical neurons. Cell Rep 7, 1168-83.

[177] De Pietri Tonelli, D., Pulvers, J.N., Haffner, C., Murchison, E.P., Hannon, G.J. and Huttner, W.B. (2008). miRNAs are essential for survival and differentiation of newborn neurons but not for expansion of neural progenitors during early neurogenesis in the mouse embryonic neocortex. Development 135, 3911-21.

[178] Davis, T.H., Cuellar, T.L., Koch, S.M., Barker, A.J., Harfe, B.D., McManus, M.T. and Ullian, E.M. (2008). Conditional loss of Dicer disrupts cellular and tissue morphogenesis in the cortex and hippocampus. J Neurosci 28, 4322-30.

[179] Cuellar, T.L., Davis, T.H., Nelson, P.T., Loeb, G.B., Harfe, B.D., Ullian, E. and McManus, M.T. (2008). Dicer loss in striatal neurons produces behavioral and neuroanatomical phenotypes in the absence of neurodegeneration. Proc Natl Acad Sci U S A 105, 5614-9.

[180] Godnic, I., Zorc, M., Jevsinek Skok, D., Calin, G.A., Horvat, S., Dovc, P., Kovac, M. and Kunej, T. (2013). Genome-wide and species-wide in silico screening for intragenic MicroRNAs in human, mouse and chicken. PLoS One 8, e65165.

[181] Monteys, A.M., Spengler, R.M., Wan, J., Tecedor, L., Lennox, K.A., Xing, Y. and Davidson, B.L. (2010). Structure and activity of putative intronic miRNA promoters. RNA 16, 495-505.

[182] Ruby, J.G., Jan, C.H. and Bartel, D.P. (2007). Intronic microRNA precursors that bypass Drosha processing. Nature 448, 83-6.

[183] Lutter, D., Marr, C., Krumsiek, J., Lang, E.W. and Theis, F.J. (2010). Intronic microRNAs support their host genes by mediating synergistic and antagonistic regulatory effects. BMC Genomics 11, 224.

[184] Hinske, L.C., Galante, P.A., Kuo, W.P. and Ohno-Machado, L. (2010). A potential role for intragenic miRNAs on their hosts' interactome. BMC Genomics 11, 533.

[185] Fukumoto, C. et al. (2014). WWP2 is overexpressed in human oral cancer, determining tumor size and poor prognosis in patients: downregulation of WWP2 inhibits the AKT signaling and tumor growth in mice. Oncoscience 1, 807-20. 
[186] Soond, S.M. and Chantry, A. (2011). Selective targeting of activating and inhibitory Smads by distinct WWP2 ubiquitin ligase isoforms differentially modulates TGFbeta signalling and EMT. Oncogene 30, 2451-62.

[187] Ahmed, S.F., Deb, S., Paul, I., Chatterjee, A., Mandal, T., Chatterjee, U. and Ghosh, M.K. (2012). The chaperone-assisted E3 ligase C terminus of Hsc70-interacting protein (CHIP) targets PTEN for proteasomal degradation. J Biol Chem 287, 159966006.

[188] Maddika, S., Kavela, S., Rani, N., Palicharla, V.R., Pokorny, J.L., Sarkaria, J.N. and Chen, J. (2011). WWP2 is an E3 ubiquitin ligase for PTEN. Nat Cell Biol 13, 72833.

[189] Xu, H., Wang, W., Li, C., Yu, H., Yang, A., Wang, B. and Jin, Y. (2009). WWP2 promotes degradation of transcription factor OCT4 in human embryonic stem cells. Cell Res 19, 561-73.

[190] Wolfson, B., Eades, G. and Zhou, Q. (2014). Roles of microRNA-140 in stem cellassociated early stage breast cancer. World J Stem Cells 6, 591-7.

[191] Li, W. et al. (2014). Down-regulation of miR-140 induces EMT and promotes invasion by targeting Slug in esophageal cancer. Cell Physiol Biochem 34, 1466-76.

[192] Zou, M.X., Huang, W., Wang, X.B., Lv, G.H., Li, J. and Deng, Y.W. (2014). Identification of miR-140-3p as a marker associated with poor prognosis in spinal chordoma. Int J Clin Exp Pathol 7, 4877-85.

[193] Li, W. and He, F. (2014). Monocyte to macrophage differentiation-associated (MMD) targeted by miR-140-5p regulates tumor growth in non-small cell lung cancer. Biochem Biophys Res Commun 450, 844-50.

[194] Kai, Y., Peng, W., Ling, W., Jiebing, H. and Zhuan, B. (2014). Reciprocal effects between microRNA-140-5p and ADAM10 suppress migration and invasion of human tongue cancer cells. Biochem Biophys Res Commun 448, 308-14.

[195] Yang, H., Fang, F., Chang, R. and Yang, L. (2013). MicroRNA-140-5p suppresses tumor growth and metastasis by targeting transforming growth factor beta receptor 1 and fibroblast growth factor 9 in hepatocellular carcinoma. Hepatology 58, 205-17.

[196] Zhai, H., Fesler, A., Ba, Y., Wu, S. and Ju, J. (2015). Inhibition of colorectal cancer stem cell survival and invasive potential by hsa-miR-140-5p mediated suppression of Smad2 and autophagy. Oncotarget 6, 19735-46.

[197] Miyaki, S. et al. (2010). MicroRNA-140 plays dual roles in both cartilage development and homeostasis. Genes Dev 24, 1173-85.

[198] Nakamura, Y. et al. (2012). Sox9 is upstream of microRNA-140 in cartilage. Appl Biochem Biotechnol 166, 64-71.

[199] Kishimoto-Suga, M. (2011) The role of HECT type ubiquitin E3 ligases WWP1 and WWP2 in nerve cell development and function. In Molecular Neurobiology ed.^eds). University of Göttingen, Göttingen.

[200] He, M., Liu, Y., Wang, X., Zhang, M.Q., Hannon, G.J. and Huang, Z.J. (2012). Celltype-based analysis of microRNA profiles in the mouse brain. Neuron 73, 35-48.

[201] Nakamura, Y., Inloes, J.B., Katagiri, T. and Kobayashi, T. (2011). Chondrocytespecific microRNA-140 regulates endochondral bone development and targets Dnpep to modulate bone morphogenetic protein signaling. Mol Cell Biol 31, 301928.

[202] Goebbels, S., Bormuth, I., Bode, U., Hermanson, O., Schwab, M.H. and Nave, K.A. (2006). Genetic targeting of principal neurons in neocortex and hippocampus of NEX-Cre mice. Genesis 44, 611-21. 
[203] Guo, H., Hong, S., Jin, X.L., Chen, R.S., Avasthi, P.P., Tu, Y.T., Ivanco, T.L. and Li, Y. (2000). Specificity and efficiency of Cre-mediated recombination in Emx1Cre knock-in mice. Biochem Biophys Res Commun 273, 661-5.

[204] Guo, W. et al. (2012). Slug and Sox9 cooperatively determine the mammary stem cell state. Cell 148, 1015-28.

[205] Mullis, K., Faloona, F., Scharf, S., Saiki, R., Horn, G. and Erlich, H. (1986). Specific enzymatic amplification of DNA in vitro: the polymerase chain reaction. Cold Spring Harb Symp Quant Biol 51 Pt 1, 263-73.

[206] Kunieda, T., Xian, M., Kobayashi, E., Imamichi, T., Moriwaki, K. and Toyoda, Y. (1992). Sexing of mouse preimplantation embryos by detection of Y chromosomespecific sequences using polymerase chain reaction. Biol Reprod 46, 692-7.

[207] Chomczynski, P. and Sacchi, N. (1987). Single-step method of RNA isolation by acid guanidinium thiocyanate-phenol-chloroform extraction. Anal Biochem 162, 156-9.

[208] Southern, E.M. (1975). Detection of specific sequences among DNA fragments separated by gel electrophoresis. J Mol Biol 98, 503-17.

[209] Fields, S. and Song, O. (1989). A novel genetic system to detect protein-protein interactions. Nature 340, 245-6.

[210] Gall, J.G. and Pardue, M.L. (1969). Formation and detection of RNA-DNA hybrid molecules in cytological preparations. Proc Natl Acad Sci U S A 63, 378-83.

[211] Summers, D.F., Maizel, J.V., Jr. and Darnell, J.E., Jr. (1965). Evidence for virusspecific noncapsid proteins in poliovirus-infected HeLa cells. Proc Natl Acad Sci U S A 54, 505-13.

[212] Laemmli, U.K. (1970). Cleavage of structural proteins during the assembly of the head of bacteriophage T4. Nature 227, 680-5.

[213] Towbin, H., Staehelin, T. and Gordon, J. (1979). Electrophoretic transfer of proteins from polyacrylamide gels to nitrocellulose sheets: procedure and some applications. Proc Natl Acad Sci U S A 76, 4350-4.

[214] Huttner, W.B., Schiebler, W., Greengard, P. and De Camilli, P. (1983). Synapsin I (protein I), a nerve terminal-specific phosphoprotein. III. Its association with synaptic vesicles studied in a highly purified synaptic vesicle preparation. J Cell Biol 96, 1374-88.

[215] Mizoguchi, A., Ueda, T., Ikeda, K., Shiku, H., Mizoguti, H. and Takai, Y. (1989). Localization and subcellular distribution of cellular ras gene products in rat brain. Brain Res Mol Brain Res 5, 31-44.

[216] Wisniewski, J.R., Zougman, A., Nagaraj, N. and Mann, M. (2009). Universal sample preparation method for proteome analysis. Nat Methods 6, 359-62.

[217] Distler, U., Kuharev, J., Navarro, P., Levin, Y., Schild, H. and Tenzer, S. (2014). Drift time-specific collision energies enable deep-coverage data-independent acquisition proteomics. Nat Methods 11, 167-70.

[218] Silva, J.C., Gorenstein, M.V., Li, G.Z., Vissers, J.P. and Geromanos, S.J. (2006). Absolute quantification of proteins by LCMSE: a virtue of parallel MS acquisition. Mol Cell Proteomics 5, 144-56.

[219] Geromanos, S.J., Hughes, C., Ciavarini, S., Vissers, J.P. and Langridge, J.I. (2012). Using ion purity scores for enhancing quantitative accuracy and precision in complex proteomics samples. Anal Bioanal Chem 404, 1127-39.

[220] Silva, J.C. et al. (2005). Quantitative proteomic analysis by accurate mass retention time pairs. Anal Chem 77, 2187-200.

[221] Li, G.Z., Vissers, J.P., Silva, J.C., Golick, D., Gorenstein, M.V. and Geromanos, S.J. (2009). Database searching and accounting of multiplexed precursor and product ion 
spectra from the data independent analysis of simple and complex peptide mixtures. Proteomics 9, 1696-719.

[222] Kuharev, J., Navarro, P., Distler, U., Jahn, O. and Tenzer, S. (2015). In-depth evaluation of software tools for data-independent acquisition based label-free quantification. Proteomics 15, 3140-51.

[223] Jiang, M. and Chen, G. (2006). High Ca2+-phosphate transfection efficiency in lowdensity neuronal cultures. Nat Protoc 1, 695-700.

[224] Gage, G.J., Kipke, D.R. and Shain, W. (2012). Whole animal perfusion fixation for rodents. J Vis Exp

[225] Yamamoto, T. and Hirano, A. (1986). A comparative study of modified Bielschowsky, Bodian and thioflavin S stains on Alzheimer's neurofibrillary tangles. Neuropathol Appl Neurobiol 12, 3-9.

[226] Matsui, A., Yoshida, A.C., Kubota, M., Ogawa, M. and Shimogori, T. (2011). Mouse in utero electroporation: controlled spatiotemporal gene transfection. J Vis Exp

[227] Paxinos G., F.K.B. (2001). The mouse brain in stereotaxic coordinates Elsevier Academic Pres

[228] WS, R. (1997-2015). ImageJ, U. S. National Institutes of Health, Behtesda, Maryland, USA, http://imagej.nih.gov/ij/.

[229] Namba, T. et al. (2014). Pioneering axons regulate neuronal polarization in the developing cerebral cortex. Neuron 81, 814-29.

[230] Shelly, M., Cancedda, L., Heilshorn, S., Sumbre, G. and Poo, M.M. (2007). LKB1/STRAD promotes axon initiation during neuronal polarization. Cell 129, 56577.

[231] Sasaki, Y. et al. (2002). Fyn and Cdk5 mediate semaphorin-3A signaling, which is involved in regulation of dendrite orientation in cerebral cortex. Neuron 35, 907-20.

[232] Yi, J.J., Barnes, A.P., Hand, R., Polleux, F. and Ehlers, M.D. (2010). TGF-beta signaling specifies axons during brain development. Cell 142, 144-57.

[233] Kishi, M., Pan, Y.A., Crump, J.G. and Sanes, J.R. (2005). Mammalian SAD kinases are required for neuronal polarization. Science 307, 929-32.

[234] Barnes, A.P., Lilley, B.N., Pan, Y.A., Plummer, L.J., Powell, A.W., Raines, A.N., Sanes, J.R. and Polleux, F. (2007). LKB1 and SAD kinases define a pathway required for the polarization of cortical neurons. Cell 129, 549-63.

[235] Berning, S., Willig, K.I., Steffens, H., Dibaj, P. and Hell, S.W. (2012). Nanoscopy in a living mouse brain. Science $335,551$.

[236] Harris, K.M., Jensen, F.E. and Tsao, B. (1992). Three-dimensional structure of dendritic spines and synapses in rat hippocampus (CA1) at postnatal day 15 and adult ages: implications for the maturation of synaptic physiology and long-term potentiation. J Neurosci 12, 2685-705.

[237] Nair, R. et al. (2013). Neurobeachin regulates neurotransmitter receptor trafficking to synapses. J Cell Biol 200, 61-80.

[238] Bischofberger, J., Engel, D., Li, L., Geiger, J.R. and Jonas, P. (2006). Patch-clamp recording from mossy fiber terminals in hippocampal slices. Nat Protoc 1, 2075-81.

[239] Craig, M.T. and McBain, C.J. (2015). Fast gamma oscillations are generated intrinsically in CA1 without the involvement of fast-spiking basket cells. J Neurosci 35, 3616-24.

[240] Dere, E. et al. (2014). Heterozygous ambra1 deficiency in mice: a genetic trait with autism-like behavior restricted to the female gender. Front Behav Neurosci 8, 181.

[241] Dere, E. et al. (2015). Gpm6b deficiency impairs sensorimotor gating and modulates the behavioral response to a 5-HT2A/C receptor agonist. Behav Brain Res 277, 25463. 
[242] El-Kordi, A. et al. (2013). A single gene defect causing claustrophobia. Transl Psychiatry 3, e254.

[243] Netrakanti, P.R., Cooper, B.H., Dere, E., Poggi, G., Winkler, D., Brose, N. and Ehrenreich, H. (2015). Fast cerebellar reflex circuitry requires synaptic vesicle priming by munc13-3. Cerebellum 14, 264-83.

[244] Ch'ng, Q., Sieburth, D. and Kaplan, J.M. (2008). Profiling synaptic proteins identifies regulators of insulin secretion and lifespan. PLoS Genet 4, e1000283.

[245] Inoue, E. et al. (2006). SAD: a presynaptic kinase associated with synaptic vesicles and the active zone cytomatrix that regulates neurotransmitter release. Neuron 50, 261-75.

[246] Crump, J.G., Zhen, M., Jin, Y. and Bargmann, C.I. (2001). The SAD-1 kinase regulates presynaptic vesicle clustering and axon termination. Neuron 29, 115-29.

[247] Campbell, K. and Gotz, M. (2002). Radial glia: multi-purpose cells for vertebrate brain development. Trends Neurosci 25, 235-8.

[248] Hatanaka, Y., Yamauchi, K. and Murakami, F. (2012). Formation of axon-dendrite polarity in situ: initiation of axons from polarized and non-polarized cells. Dev Growth Differ 54, 398-407.

[249] Rakic, P. (1971). Neuron-glia relationship during granule cell migration in developing cerebellar cortex. A Golgi and electronmicroscopic study in Macacus Rhesus. J Comp Neurol 141, 283-312.

[250] Rakic, P. (1972). Mode of cell migration to the superficial layers of fetal monkey neocortex. J Comp Neurol 145, 61-83.

[251] Kriegstein, A.R. and Noctor, S.C. (2004). Patterns of neuronal migration in the embryonic cortex. Trends Neurosci 27, 392-9.

[252] Saito, T. and Nakatsuji, N. (2001). Efficient gene transfer into the embryonic mouse brain using in vivo electroporation. Dev Biol 240, 237-46.

[253] Marin-Padilla, M. (2014). The mammalian neocortex new pyramidal neuron: a new conception. Front Neuroanat 7, 51.

[254] Horvitz, H.R. and Sulston, J.E. (1980). Isolation and genetic characterization of celllineage mutants of the nematode Caenorhabditis elegans. Genetics 96, 435-54.

[255] Lee, Y., Kim, M., Han, J., Yeom, K.H., Lee, S., Baek, S.H. and Kim, V.N. (2004). MicroRNA genes are transcribed by RNA polymerase II. EMBO J 23, 4051-60.

[256] Hinske, L.C. et al. (2014). miRIAD-integrating microRNA inter- and intragenic data. Database (Oxford) 2014

[257] Dill, H., Linder, B., Fehr, A. and Fischer, U. (2012). Intronic miR-26b controls neuronal differentiation by repressing its host transcript, ctdsp2. Genes Dev 26, 2530.

[258] Li, Q., Yao, Y., Eades, G., Liu, Z., Zhang, Y. and Zhou, Q. (2014). Downregulation of miR-140 promotes cancer stem cell formation in basal-like early stage breast cancer. Oncogene 33, 2589-600.

[259] Lee, Y., Jeon, K., Lee, J.T., Kim, S. and Kim, V.N. (2002). MicroRNA maturation: stepwise processing and subcellular localization. EMBO J 21, 4663-70.

[260] Melo, S.A. et al. (2009). A TARBP2 mutation in human cancer impairs microRNA processing and DICER1 function. Nat Genet 41, 365-70.

[261] Schratt, G.M., Tuebing, F., Nigh, E.A., Kane, C.G., Sabatini, M.E., Kiebler, M. and Greenberg, M.E. (2006). A brain-specific microRNA regulates dendritic spine development. Nature 439, 283-9.

[262] Han, J. et al. (2006). Molecular basis for the recognition of primary microRNAs by the Drosha-DGCR8 complex. Cell 125, 887-901. 
[263] Schwarz, D.S., Hutvagner, G., Du, T., Xu, Z., Aronin, N. and Zamore, P.D. (2003). Asymmetry in the assembly of the RNAi enzyme complex. Cell 115, 199-208.

[264] Chiang, H.R. et al. (2010). Mammalian microRNAs: experimental evaluation of novel and previously annotated genes. Genes Dev 24, 992-1009.

[265] Li, J., Yang, Z., Yu, B., Liu, J. and Chen, X. (2005). Methylation protects miRNAs and siRNAs from a 3'-end uridylation activity in Arabidopsis. Curr Biol 15, 1501-7.

[266] Kamminga, L.M. et al. (2012). Differential impact of the HEN1 homolog HENN-1 on 21U and 26G RNAs in the germline of Caenorhabditis elegans. PLoS Genet 8, e1002702.

[267] Kertesz, M., Iovino, N., Unnerstall, U., Gaul, U. and Segal, E. (2007). The role of site accessibility in microRNA target recognition. Nat Genet 39, 1278-84.

[268] (1970). Embryonic vertebrate central nervous system: revised terminology. The Boulder Committee. Anat Rec 166, 257-61.

[269] Shi, Z., Luo, G., Fu, L., Fang, Z., Wang, X. and Li, X. (2013). miR-9 and miR-140$5 p$ target FoxP2 and are regulated as a function of the social context of singing behavior in zebra finches. J Neurosci 33, 16510-21.

[270] Chiang, D.Y., Cuthbertson, D.W., Ruiz, F.R., Li, N. and Pereira, F.A. (2013). A coregulatory network of NR2F1 and microRNA-140. PLoS One 8, e83358.

[271] Zhang, Y., Eades, G., Yao, Y., Li, Q. and Zhou, Q. (2012). Estrogen receptor alpha signaling regulates breast tumor-initiating cells by down-regulating miR-140 which targets the transcription factor SOX2. J Biol Chem 287, 41514-22.

[272] Valinezhad Orang, A., Safaralizadeh, R. and Kazemzadeh-Bavili, M. (2014). Mechanisms of miRNA-Mediated Gene Regulation from Common Downregulation to mRNA-Specific Upregulation. Int J Genomics 2014, 970607.

[273] Hausser, J. and Zavolan, M. (2014). Identification and consequences of miRNAtarget interactions--beyond repression of gene expression. Nat Rev Genet 15, 599612.

[274] Yamashita, S. et al. (2012). L-Sox5 and Sox6 proteins enhance chondrogenic miR140 microRNA expression by strengthening dimeric Sox 9 activity. J Biol Chem 287, 22206-15.

[275] Kang, P. et al. (2012). Sox 9 and NFIA coordinate a transcriptional regulatory cascade during the initiation of gliogenesis. Neuron 74, 79-94.

[276] Akiyama, H., Chaboissier, M.C., Martin, J.F., Schedl, A. and de Crombrugghe, B. (2002). The transcription factor Sox9 has essential roles in successive steps of the chondrocyte differentiation pathway and is required for expression of Sox 5 and Sox6. Genes Dev 16, 2813-28.

[277] Akiyama, H., Chaboissier, M.C., Behringer, R.R., Rowitch, D.H., Schedl, A., Epstein, J.A. and de Crombrugghe, B. (2004). Essential role of Sox9 in the pathway that controls formation of cardiac valves and septa. Proc Natl Acad Sci U S A 101, 6502-7.

[278] Arlotta, P., Molyneaux, B.J., Chen, J., Inoue, J., Kominami, R. and Macklis, J.D. (2005). Neuronal subtype-specific genes that control corticospinal motor neuron development in vivo. Neuron 45, 207-21.

[279] Angevine, J.B., Jr. (1965). Time of neuron origin in the hippocampal region. An autoradiographic study in the mouse. Exp Neurol Suppl, Suppl 2:1-70.

[280] Tole, S., Goudreau, G., Assimacopoulos, S. and Grove, E.A. (2000). Emx2 is required for growth of the hippocampus but not for hippocampal field specification. J Neurosci 20, 2618-25. 
[281] Song, J.Y., Ichtchenko, K., Sudhof, T.C. and Brose, N. (1999). Neuroligin 1 is a postsynaptic cell-adhesion molecule of excitatory synapses. Proc Natl Acad Sci U S A 96, 1100-5.

[282] Sieburth, D. et al. (2005). Systematic analysis of genes required for synapse structure and function. Nature 436, 510-7.

[283] Dibbens, L.M. et al. (2007). NEDD4-2 as a potential candidate susceptibility gene for epileptic photosensitivity. Genes Brain Behav 6, 750-5.

[284] Tarpey, P.S. et al. (2009). A systematic, large-scale resequencing screen of Xchromosome coding exons in mental retardation. Nat Genet 41, 535-43.

[285] Loukas, M., Shayota, B.J., Oelhafen, K., Miller, J.H., Chern, J.J., Tubbs, R.S. and Oakes, W.J. (2011). Associated disorders of Chiari Type I malformations: a review. Neurosurg Focus 31, E3.

[286] Ferrer, I. and Gullotta, F. (1990). Down's syndrome and Alzheimer's disease: dendritic spine counts in the hippocampus. Acta Neuropathol 79, 680-5.

[287] Marin-Padilla, M. (1972). Structural abnormalities of the cerebral cortex in human chromosomal aberrations: a Golgi study. Brain Res 44, 625-9.

[288] Belichenko, P.V., Oldfors, A., Hagberg, B. and Dahlstrom, A. (1994). Rett syndrome: 3-D confocal microscopy of cortical pyramidal dendrites and afferents. Neuroreport 5, 1509-13.

[289] Wang, Q., Moore, M.J., Adelmant, G., Marto, J.A. and Silver, P.A. (2013). PQBP1, a factor linked to intellectual disability, affects alternative splicing associated with neurite outgrowth. Genes Dev 27, 615-26.

[290] Sholl, D.A. (1953). Dendritic organization in the neurons of the visual and motor cortices of the cat. J Anat 87, 387-406.

[291] Jin, X.L., Guo, H., Mao, C., Atkins, N., Wang, H., Avasthi, P.P., Tu, Y.T. and Li, Y. (2000). Emx1-specific expression of foreign genes using "knock-in" approach. Biochem Biophys Res Commun 270, 978-82.

[292] Khan, O.H., Enno, T.L. and Del Bigio, M.R. (2006). Brain damage in neonatal rats following kaolin induction of hydrocephalus. Exp Neurol 200, 311-20.

[293] Kurt, G., Cemil, B., Borcek, A.O., Borcek, P., Akyurek, N., Sepici, A. and Ceviker, N. (2010). Infliximab administration reduces neuronal apoptosis on the optic pathways in a rabbit hydrocephalus model: a preliminary report. Br J Neurosurg 24, 275-9.

[294] Lang, B., Song, B., Davidson, W., MacKenzie, A., Smith, N., McCaig, C.D., Harmar, A.J. and Shen, S. (2006). Expression of the human PAC1 receptor leads to dosedependent hydrocephalus-related abnormalities in mice. J Clin Invest 116, 1924-34.

[295] Dindot, S.V., Antalffy, B.A., Bhattacharjee, M.B. and Beaudet, A.L. (2008). The Angelman syndrome ubiquitin ligase localizes to the synapse and nucleus, and maternal deficiency results in abnormal dendritic spine morphology. Hum Mol Genet 17, 111-8.

[296] Chapleau, C.A. et al. (2009). Dendritic spine pathologies in hippocampal pyramidal neurons from Rett syndrome brain and after expression of Rett-associated MECP2 mutations. Neurobiol Dis 35, 219-33.

[297] Bassani, S. et al. (2012). The X-linked intellectual disability protein TSPAN7 regulates excitatory synapse development and AMPAR trafficking. Neuron 73, 1143-58.

[298] Bekkers, J.M. and Stevens, C.F. (1991). Excitatory and inhibitory autaptic currents in isolated hippocampal neurons maintained in cell culture. Proc Natl Acad Sci U S A $88,7834-8$. 
[299] Tsien, J.Z., Huerta, P.T. and Tonegawa, S. (1996). The essential role of hippocampal CA1 NMDA receptor-dependent synaptic plasticity in spatial memory. Cell 87, 1327-38.

[300] Kim, H.C. and Huibregtse, J.M. (2009). Polyubiquitination by HECT E3s and the determinants of chain type specificity. Mol Cell Biol 29, 3307-18.

[301] Michel, M.A., Elliott, P.R., Swatek, K.N., Simicek, M., Pruneda, J.N., Wagstaff, J.L., Freund, S.M. and Komander, D. (2015). Assembly and specific recognition of k29and k33-linked polyubiquitin. Mol Cell 58, 95-109.

[302] Choi, Y.J., Di Nardo, A., Kramvis, I., Meikle, L., Kwiatkowski, D.J., Sahin, M. and He, X. (2008). Tuberous sclerosis complex proteins control axon formation. Genes Dev 22, 2485-95.

[303] Jiang, H., Guo, W., Liang, X. and Rao, Y. (2005). Both the establishment and the maintenance of neuronal polarity require active mechanisms: critical roles of GSK3beta and its upstream regulators. Cell 120, 123-35.

[304] Chen, T., Wu, Q., Zhang, Y. and Zhang, D. (2015). NDUFV2 regulates neuronal migration in the developing cerebral cortex through modulation of the multipolarbipolar transition. Brain Res

[305] La Fata, G. et al. (2014). FMRP regulates multipolar to bipolar transition affecting neuronal migration and cortical circuitry. Nat Neurosci 17, 1693-700.

[306] Gonda, Y. et al. (2013). Robol regulates the migration and laminar distribution of upper-layer pyramidal neurons of the cerebral cortex. Cereb Cortex 23, 1495-508.

[307] Polleux, F., Morrow, T. and Ghosh, A. (2000). Semaphorin 3A is a chemoattractant for cortical apical dendrites. Nature 404, 567-73.

[308] Asada, N., Sanada, K. and Fukada, Y. (2007). LKB1 regulates neuronal migration and neuronal differentiation in the developing neocortex through centrosomal positioning. J Neurosci 27, 11769-75.

[309] Voss, A.K., Britto, J.M., Dixon, M.P., Sheikh, B.N., Collin, C., Tan, S.S. and Thomas, T. (2008). C3G regulates cortical neuron migration, preplate splitting and radial glial cell attachment. Development 135, 2139-49.

[310] Bedford, M.T., Chan, D.C. and Leder, P. (1997). FBP WW domains and the Abl SH3 domain bind to a specific class of proline-rich ligands. EMBO J 16, 2376-83.

[311] Chen, H.I. and Sudol, M. (1995). The WW domain of Yes-associated protein binds a proline-rich ligand that differs from the consensus established for Src homology 3binding modules. Proc Natl Acad Sci U S A 92, 7819-23.

[312] Thomas, M., Lieberman, J. and Lal, A. (2010). Desperately seeking microRNA targets. Nat Struct Mol Biol 17, 1169-74.

[313] Giusti, S.A. et al. (2014). MicroRNA-9 controls dendritic development by targeting REST. Elife 3

[314] Yoo, A.S. et al. (2011). MicroRNA-mediated conversion of human fibroblasts to neurons. Nature 476, 228-31.

[315] Mellios, N. et al. (2011). miR-132, an experience-dependent microRNA, is essential for visual cortex plasticity. Nat Neurosci 14, 1240-2.

[316] Siegert, S. et al. (2015). The schizophrenia risk gene product miR-137 alters presynaptic plasticity. Nat Neurosci 18, 1008-16.

[317] Wienholds, E. et al. (2005). MicroRNA expression in zebrafish embryonic development. Science 309, 310-1.

[318] Tuddenham, L., Wheeler, G., Ntounia-Fousara, S., Waters, J., Hajihosseini, M.K., Clark, I. and Dalmay, T. (2006). The cartilage specific microRNA-140 targets histone deacetylase 4 in mouse cells. FEBS Lett 580, 4214-7. 
[319] Zhang, R., Ma, J. and Yao, J. (2013). Molecular mechanisms of the cartilage-specific microRNA-140 in osteoarthritis. Inflamm Res 62, 871-7.

[320] Lee, Y.S. et al. (2009). Silencing by small RNAs is linked to endosomal trafficking. Nat Cell Biol 11, 1150-6.

[321] Gibbings, D.J., Ciaudo, C., Erhardt, M. and Voinnet, O. (2009). Multivesicular bodies associate with components of miRNA effector complexes and modulate miRNA activity. Nat Cell Biol 11, 1143-9.

[322] Hancock, M.L., Preitner, N., Quan, J. and Flanagan, J.G. (2014). MicroRNA-132 is enriched in developing axons, locally regulates Rasa1 mRNA, and promotes axon extension. J Neurosci 34, 66-78.

[323] Mattila, P.K. and Lappalainen, P. (2008). Filopodia: molecular architecture and cellular functions. Nat Rev Mol Cell Biol 9, 446-54.

[324] Fiumelli, H., Riederer, I.M., Martin, J.L. and Riederer, B.M. (2008). Phosphorylation of neurofilament subunit NF-M is regulated by activation of NMDA receptors and modulates cytoskeleton stability and neuronal shape. Cell Motil Cytoskeleton 65, 495-504.

[325] Sanchez, I., Hassinger, L., Sihag, R.K., Cleveland, D.W., Mohan, P. and Nixon, R.A. (2000). Local control of neurofilament accumulation during radial growth of myelinating axons in vivo. Selective role of site-specific phosphorylation. J Cell Biol 151, 1013-24.

[326] Baek, D., Villen, J., Shin, C., Camargo, F.D., Gygi, S.P. and Bartel, D.P. (2008). The impact of microRNAs on protein output. Nature 455, 64-71.

[327] Ambros, V. (2004). The functions of animal microRNAs. Nature 431, 350-5.

[328] Selbach, M., Schwanhausser, B., Thierfelder, N., Fang, Z., Khanin, R. and Rajewsky, N. (2008). Widespread changes in protein synthesis induced by microRNAs. Nature $455,58-63$.

[329] Tan, C.L. et al. (2013). MicroRNA-128 governs neuronal excitability and motor behavior in mice. Science 342, 1254-8.

[330] Maier, T., Guell, M. and Serrano, L. (2009). Correlation of mRNA and protein in complex biological samples. FEBS Lett 583, 3966-73.

[331] Akiyama, H. et al. (2004). Interactions between Sox9 and beta-catenin control chondrocyte differentiation. Genes Dev 18, 1072-87.

[332] Li, M., Cui, Z., Niu, Y., Liu, B., Fan, W., Yu, D. and Deng, J. (2010). Synaptogenesis in the developing mouse visual cortex. Brain Res Bull 81, 107-13.

[333] Huttenlocher, P.R. (1984). Synapse elimination and plasticity in developing human cerebral cortex. Am J Ment Defic 88, 488-96.

[334] Fiala, J.C., Spacek, J. and Harris, K.M. (2002). Dendritic spine pathology: cause or consequence of neurological disorders? Brain Res Brain Res Rev 39, 29-54.

[335] Comery, T.A., Harris, J.B., Willems, P.J., Oostra, B.A., Irwin, S.A., Weiler, I.J. and Greenough, W.T. (1997). Abnormal dendritic spines in fragile X knockout mice: maturation and pruning deficits. Proc Natl Acad Sci U S A 94, 5401-4.

[336] Noguchi, J., Matsuzaki, M., Ellis-Davies, G.C. and Kasai, H. (2005). Spine-neck geometry determines NMDA receptor-dependent $\mathrm{Ca} 2+$ signaling in dendrites. Neuron 46, 609-22.

[337] Fischer, M., Kaech, S., Knutti, D. and Matus, A. (1998). Rapid actin-based plasticity in dendritic spines. Neuron 20, 847-54.

[338] Harris, K.M. and Stevens, J.K. (1989). Dendritic spines of CA 1 pyramidal cells in the rat hippocampus: serial electron microscopy with reference to their biophysical characteristics. J Neurosci 9, 2982-97. 
[339] Volfovsky, N., Parnas, H., Segal, M. and Korkotian, E. (1999). Geometry of dendritic spines affects calcium dynamics in hippocampal neurons: theory and experiments. J Neurophysiol 82, 450-62.

[340] Araya, R., Vogels, T.P. and Yuste, R. (2014). Activity-dependent dendritic spine neck changes are correlated with synaptic strength. Proc Natl Acad Sci U S A 111, E2895-904.

[341] Rusakov, D.A., Richter-Levin, G., Stewart, M.G. and Bliss, T.V. (1997). Reduction in spine density associated with long-term potentiation in the dentate gyrus suggests a spine fusion-and-branching model of potentiation. Hippocampus 7, 489-500.

[342] Hering, H. and Sheng, M. (2001). Dendritic spines: structure, dynamics and regulation. Nat Rev Neurosci 2, 880-8.

[343] Persaud, A. et al. (2009). Comparison of substrate specificity of the ubiquitin ligases Nedd4 and Nedd4-2 using proteome arrays. Mol Syst Biol 5, 333.

[344] Albrecht, U., Sutcliffe, J.S., Cattanach, B.M., Beechey, C.V., Armstrong, D., Eichele, G. and Beaudet, A.L. (1997). Imprinted expression of the murine Angelman syndrome gene, Ube3a, in hippocampal and Purkinje neurons. Nat Genet 17, 75-8.

[345] Waragai, M. et al. (1999). PQBP-1, a novel polyglutamine tract-binding protein, inhibits transcription activation by Brn-2 and affects cell survival. Hum Mol Genet 8, 977-87.

[346] Dragich, J.M., Kim, Y.H., Arnold, A.P. and Schanen, N.C. (2007). Differential distribution of the $\mathrm{MeCP} 2$ splice variants in the postnatal mouse brain. J Comp Neurol 501, 526-42.

[347] Murata, Y. and Constantine-Paton, M. (2013). Postsynaptic density scaffold SAP102 regulates cortical synapse development through EphB and PAK signaling pathway. J Neurosci 33, 5040-52.

[348] Chen, X. et al. (2011). PSD-95 is required to sustain the molecular organization of the postsynaptic density. J Neurosci 31, 6329-38.

[349] Migaud, M. et al. (1998). Enhanced long-term potentiation and impaired learning in mice with mutant postsynaptic density-95 protein. Nature 396, 433-9.

[350] Willig, K.I., Harke, B., Medda, R. and Hell, S.W. (2007). STED microscopy with continuous wave beams. Nat Methods 4, 915-8.

[351] Hell, S.W. and Wichmann, J. (1994). Breaking the diffraction resolution limit by stimulated emission: stimulated-emission-depletion fluorescence microscopy. Opt Lett 19, 780-2.

[352] Agarwal, A. et al. (2014). Dysregulated expression of neuregulin-1 by cortical pyramidal neurons disrupts synaptic plasticity. Cell Rep 8, 1130-45.

[353] Kalscheuer, V.M. et al. (2003). Mutations in the polyglutamine binding protein 1 gene cause X-linked mental retardation. Nat Genet 35, 313-5.

[354] Stevenson, R.E. et al. (2005). Renpenning syndrome comes into focus. Am J Med Genet A 134, 415-21.

[355] Fukuda, T., Itoh, M., Ichikawa, T., Washiyama, K. and Goto, Y. (2005). Delayed maturation of neuronal architecture and synaptogenesis in cerebral cortex of Mecp2deficient mice. J Neuropathol Exp Neurol 64, 537-44.

[356] Kishi, N. and Macklis, J.D. (2004). MECP2 is progressively expressed in postmigratory neurons and is involved in neuronal maturation rather than cell fate decisions. Mol Cell Neurosci 27, 306-21.

[357] Armstrong, D., Dunn, J.K., Antalffy, B. and Trivedi, R. (1995). Selective dendritic alterations in the cortex of Rett syndrome. J Neuropathol Exp Neurol 54, 195-201.

[358] Hausser, M., Spruston, N. and Stuart, G.J. (2000). Diversity and dynamics of dendritic signaling. Science 290, 739-44. 
[359] Mainen, Z.F. and Sejnowski, T.J. (1996). Influence of dendritic structure on firing pattern in model neocortical neurons. Nature 382, 363-6.

[360] Friedlander, M.J., Stanford, L.R. and Sherman, S.M. (1982). Effects of monocular deprivation on the structure-function relationship of individual neurons in the cat's lateral geniculate nucleus. J Neurosci 2, 321-30.

[361] Bacon, J.P. and Murphey, R.K. (1984). Receptive fields of cricket giant interneurones are related to their dendritic structure. J Physiol 352, 601-23.

[362] Dierssen, M. and Ramakers, G.J. (2006). Dendritic pathology in mental retardation: from molecular genetics to neurobiology. Genes Brain Behav 5 Suppl 2, 48-60.

[363] Ramakers, G.J. (2000). Rho proteins and the cellular mechanisms of mental retardation. Am J Med Genet 94, 367-71.

[364] Ramakers, G.J. (2002). Rho proteins, mental retardation and the cellular basis of cognition. Trends Neurosci 25, 191-9.

[365] Fischer, M., Kaech, S., Wagner, U., Brinkhaus, H. and Matus, A. (2000). Glutamate receptors regulate actin-based plasticity in dendritic spines. Nat Neurosci 3, 887-94.

[366] Engert, F. and Bonhoeffer, T. (1999). Dendritic spine changes associated with hippocampal long-term synaptic plasticity. Nature 399, 66-70.

[367] Maletic-Savatic, M., Malinow, R. and Svoboda, K. (1999). Rapid dendritic morphogenesis in CA1 hippocampal dendrites induced by synaptic activity. Science 283, 1923-7.

[368] Toni, N., Buchs, P.A., Nikonenko, I., Bron, C.R. and Muller, D. (1999). LTP promotes formation of multiple spine synapses between a single axon terminal and a dendrite. Nature 402, 421-5.

[369] Reeves, R.H. et al. (1995). A mouse model for Down syndrome exhibits learning and behaviour deficits. Nat Genet 11, 177-84.

[370] Belichenko, P.V., Masliah, E., Kleschevnikov, A.M., Villar, A.J., Epstein, C.J., Salehi, A. and Mobley, W.C. (2004). Synaptic structural abnormalities in the Ts65Dn mouse model of Down Syndrome. J Comp Neurol 480, 281-98.

[371] Kurt, M.A., Davies, D.C., Kidd, M., Dierssen, M. and Florez, J. (2000). Synaptic deficit in the temporal cortex of partial trisomy 16 (Ts65Dn) mice. Brain Res 858, 191-7.

[372] Belichenko, P.V., Wright, E.E., Belichenko, N.P., Masliah, E., Li, H.H., Mobley, W.C. and Francke, U. (2009). Widespread changes in dendritic and axonal morphology in Mecp2-mutant mouse models of Rett syndrome: evidence for disruption of neuronal networks. J Comp Neurol 514, 240-58.

[373] Smrt, R.D., Eaves-Egenes, J., Barkho, B.Z., Santistevan, N.J., Zhao, C., Aimone, J.B., Gage, F.H. and Zhao, X. (2007). Mecp2 deficiency leads to delayed maturation and altered gene expression in hippocampal neurons. Neurobiol Dis 27, 77-89.

[374] Fu, Y.H. et al. (1991). Variation of the CGG repeat at the fragile X site results in genetic instability: resolution of the Sherman paradox. Cell 67, 1047-58.

[375] Irwin, S.A., Galvez, R. and Greenough, W.T. (2000). Dendritic spine structural anomalies in fragile-X mental retardation syndrome. Cereb Cortex 10, 1038-44.

[376] McKinney, B.C., Grossman, A.W., Elisseou, N.M. and Greenough, W.T. (2005). Dendritic spine abnormalities in the occipital cortex of C57BL/6 Fmr1 knockout mice. Am J Med Genet B Neuropsychiatr Genet 136B, 98-102.

[377] McLeod, F., Ganley, R., Williams, L., Selfridge, J., Bird, A. and Cobb, S.R. (2013). Reduced seizure threshold and altered network oscillatory properties in a mouse model of Rett syndrome. Neuroscience 231, 195-205.

[378] Woo, T.U., Spencer, K. and McCarley, R.W. (2010). Gamma oscillation deficits and the onset and early progression of schizophrenia. Harv Rev Psychiatry 18, 173-89. 
[379] Rojas, D.C. and Wilson, L.B. (2014). gamma-band abnormalities as markers of autism spectrum disorders. Biomark Med 8, 353-68.

[380] Hammer, M. (2012) Characterization of neuroligin 4, a protein involved in autism spectrum disorders. In Molecular Neurobiology ed.^eds). Uni Göttingen, MPIem.

[381] Faizi, M. et al. (2011). Comprehensive behavioral phenotyping of Ts65Dn mouse model of Down syndrome: activation of beta1-adrenergic receptor by xamoterol as a potential cognitive enhancer. Neurobiol Dis 43, 397-413.

[382] Khelfaoui, M. et al. (2007). Loss of X-linked mental retardation gene oligophrenin1 in mice impairs spatial memory and leads to ventricular enlargement and dendritic spine immaturity. J Neurosci 27, 9439-50.

[383] Blundell, J. et al. (2010). Neuroligin-1 deletion results in impaired spatial memory and increased repetitive behavior. J Neurosci 30, 2115-29.

[384] Hitti, F.L. and Siegelbaum, S.A. (2014). The hippocampal CA2 region is essential for social memory. Nature 508, 88-92.

[385] Csicsvari, J., Jamieson, B., Wise, K.D. and Buzsaki, G. (2003). Mechanisms of gamma oscillations in the hippocampus of the behaving rat. Neuron 37, 311-22.

[386] Buzsaki, G., Buhl, D.L., Harris, K.D., Csicsvari, J., Czeh, B. and Morozov, A. (2003). Hippocampal network patterns of activity in the mouse. Neuroscience 116, 201-11.

[387] Fuchs, E.C. et al. (2007). Recruitment of parvalbumin-positive interneurons determines hippocampal function and associated behavior. Neuron 53, 591-604.

[388] Ma, J. and Leung, L.S. (2000). Relation between hippocampal gamma waves and behavioral disturbances induced by phencyclidine and methamphetamine. Behav Brain Res 111, 1-11.

[389] Santosh, P.J. and Baird, G. (1999). Psychopharmacotherapy in children and adults with intellectual disability. Lancet 354, 233-42.

[390] Wing, L. and Gould, J. (1979). Severe impairments of social interaction and associated abnormalities in children: epidemiology and classification. J Autism Dev Disord 9, 11-29.

[391] Tantra, M. et al. (2014). Mild expression differences of MECP2 influencing aggressive social behavior. EMBO Mol Med 6, 662-84.

[392] Peca, J. et al. (2011). Shank3 mutant mice display autistic-like behaviours and striatal dysfunction. Nature 472, 437-42.

[393] Tabuchi, K., Blundell, J., Etherton, M.R., Hammer, R.E., Liu, X., Powell, C.M. and Sudhof, T.C. (2007). A neuroligin-3 mutation implicated in autism increases inhibitory synaptic transmission in mice. Science 318, 71-6.

[394] Zhang, H., Webb, D.J., Asmussen, H., Niu, S. and Horwitz, A.F. (2005). A GIT1/PIX/Rac/PAK signaling module regulates spine morphogenesis and synapse formation through MLC. J Neurosci 25, 3379-88.

[395] Wu, H.Y. et al. (2012). Distinct dendritic spine and nuclear phases of calcineurin activation after exposure to amyloid-beta revealed by a novel fluorescence resonance energy transfer assay. J Neurosci 32, 5298-309.

[396] Halpain, S., Hipolito, A. and Saffer, L. (1998). Regulation of F-actin stability in dendritic spines by glutamate receptors and calcineurin. J Neurosci 18, 9835-44. 


\section{ACKNOWLEDGEMENTS}

I would like to express my gratitude toward all the people who made this work possible, and who contributed to its success. First, I thank Prof. Nils Brose for including me as a member of Department of Molecular Neurobiology, his undaunted trust and constant encouragement. Nils, thank you for pushing me to become a fearless explorer.

I would like to express a special gratitude towards Dr. Hiroshi Kawabe, who supervised projects describe in this thesis. Hiroshi, thank you for your unbroken perseverance, long scientific discussions, and all the time you devoted for my scientific growth. Above all, thank you for making me devoted to science.

Importantly, I would like to express my gratitude towards members of the thesis committee, Dr. Judith Stegmüller, and Prof. Ahmed Mansouri for valuable scientific input, and their contribution to progress of my projects.

I sincerely thank Dr. Craig Don Paul for proof reading of this manuscript and Dr. Hiroshi Kawabe for all insightful comments and remarks.

This work would not be accomplished without the collaborators. First, and foremost, I sincerely thank Dr. Silvia Ripamonti and Dr. Jeong Seop Rhee for their laborious, yet elegant, and professional scientific input to the characterization of Ube3b in neurons and in the brain. I would like to thank Prof. Ekrem Dere and Prof. Hannelore Ehrenreich for thorough characterization of $U b e 3 b \mathrm{cKO}$ mouse behavior, without which this project would be significantly incomplete. Further, I thank Dr. Katrin Willig for introducing me to the world of STED nanoscopy, and vibrant and motivating discussions about morphometrics. I thank Dr. Olaf Jahn for his invaluable expertise and involvement in characterization of miR140 targets and Ube3b substrates. Next, I would like to thank Prof. Haruhiko Akiyama and Dr. Mikio Hoshino for experimental help in characterization of Sox $9 \mathrm{KO}$ in neurons. Further, I thank Dr. Tamara Rabe and Dr. Xin Zhang for help with characterization of $m i R-140$ expression in neurons. I would also like to thank Fritz Benseler for his priceless help and expertise in the field of nucleic acids biology. Last but not least, I thank Bekir Altas for his invaluable expertise in protein purification and subcellular fractionation of the brain. 
I would like to express my biggest gratitude towards IMPRS Neuroscience coordination office, Sandra Drube and Prof. Michael Hörner. Sandra, you (very professionally every time) have made my life easier in the most stressful situations. Michael, I will never forget how dumbfounded I was when during the interview you asked me 'so what exactly is memory?'. I still do not know, but I am sure my time at IMPRS Neuroscience has brought me closer to the answer.

Significant part of this project would not be possible without technical assistance of Klaus Hellmann. Klaus, Danke schön für deine Unterstützung und große Hilfe.

An exceptional thanks to Manuela Schwark for her unprecedented professionalism, patience, and physical and mental support throughout this project. Manu, it is not only the scientific skills you thought me, but you also showed me how to find a way out of every madness.

I would like to express my greatest gratitude for the assistance of all members of AGCT lab, who made this work much faster and more efficient. Great thanks to Dr. Ursula Fünfschilling, Ing. Rainer Libal, and Monika Schindler for their expertise and mouse work. I would like to express a special gratitude towards animal caretakers, Sabrina, Nadine, Steffi for their patient support and hard work.

I would like to acknowledge the support of all members of Department of Molecular Neurobiology throughout my doctorate. Special thanks to Bekir, Marylin, Matthieu, Ben, Cordelia, Rikka, Dilja, James, Mimi, Anja, Olga, Noa, Theo, Caro, and Sünke for encouragement, scientific support, and sometimes a motivating slap during times of despair.

Last but not least, I thank my 'German family' for everything. Siv, Marta, Irena, Jan, Goran, Paola, Victor this was a long, bumpy, and exhausting road, but you made it with me. Craig, Katrina, our friendship started quickly, and spontaneously, but it grew bigger than RuPaul's hair. Silvia, you will always be the special queen in my heart. Daniel, as you once said, we are family, brother.

Chciałbym także najszczerzej podziękować mojej najdroższej Martynie za sto lat lojalnej przyjaźni. Dziękuję też całej Ekypie, przede wszystkim Oli U., Oli M., Agnieszce, Kaśce B., Jackowi, Kamilowi, Kini i Mateuszowi za wsparcie i za kciuki przez te 5 lat! 
Ten doktorat nie byłby w ogóle możliwy bez mojej najlepszej na świecie rodzinki. Dziękuję Wam, Mamo, Natalio, Macieju, Januszu i dziadku Jurku za to, że wytrwaliście ze mną przez ten czas i że zawsze mogłem na Was liczyć. 


\section{LIST OF PUBLICATIONS}

Ripamonti S, Ambrozkiewicz MC, Guzzi F, Gravati M, Biella G, Busnelli M, Parthasarathy S, Hammer M, Kawabe H, Nishimori K, Chini B, Toselli M, Brose N, Parenti M, Rhee JS. Oxytocin Regulates Hippocampal Function and Neuronal Networking. Manuscript in preparation.

Ambrozkiewicz MC, Kawabe H. HECT-type ubiquitin ligases in the nerve cell development and synapse physiology. FEBS Lett. 2015 Jun; 589(14):1635-43.

Misztal K, Wisniewska MB, Ambrozkiewicz M, Kuznicki J. Wnt-independent constitutive nuclear localization of $\beta$-catenin and its low degradation rate in thalamic neurons. J Biol Chem. 2011 Sep; 286(36):31781-8.

Michowski W, Ferretti R, Wisniewska MB, Ambrozkiewicz M, Beresewicz M, Fusella F, Skibinska-Kijek A, Zablocka B, Brancaccio M, Tarone G, Kuznicki J. Morgana/CHP-1 is a novel chaperone able to protect cells from stress. Biochim Biophys Acta. 2010 Sep;1803(9):1043-9. 


\section{RESUMÉ}

Full name:

Address:

Telephone:

Date of birth:

Nationality:

\section{Education}

2010-2015

2006-2010
Mateusz Cyryl Ambrożkiewicz

Department of Molecular Neurobiology

Hermann-Rein-Straße 3, 37075 Göttingen, Germany

$+49551 / 3899718$

27.06.1987

Polish

$\mathrm{PhD}$ at Georg-August Universität Göttingen

International Max Planck Research School Neurosciences

Max Planck Institute of Experimental Medicine

Department of Molecular Biology

Thesis: 'HECT-type Ubiquitin Ligases in the Nerve Cell Development', supervised by Dr. Hiroshi Kawabe and Prof. Nils Brose

Engineer at Warsaw University of Life Sciences in Biotechnology

International Institute of Molecular and Cell Biology in Warsaw

Laboratory of Neurodegeneration

Thesis: 'Stress-regulated expression of Morgana/Chp-1 - a gene for a novel chaperone-like protein', supervised by Dr. Wojciech Michowski and Prof. Jacek Kuźnicki

\section{Research Projects}

2011 Lab Rotation 'The distribution of neuroligin-3 in the mouse central nervous system', supervised by Dr. Matthieu Hammer and Dr. Dilja Krüger-Burg, Max Planck Institute of Experimental Medicine, Department of Molecular Biology, Göttingen

Lab Rotation 'Neuromuscular specification: coordinating motor neurons and muscle fiber properties', supervised by Dr. Anne Poh and Dr. Till Marquardt, Developmental Neurobiology, European Neurosciences Institute, Göttingen

Lab Rotation 'Substrate vibration affects walking behavior in Drosophila melanogaster', supervised by Dr. Robert Wiek and Professor Martin Göpfert, Cellular Neurobiology Department, Georg-August University of Göttingen

2010 Research Assistant 'The analysis of the canonical Wnt signal transduction pathway in the adult rat brain', supervised by Dr. Katarzyna Misztal and Dr. Marta Wiśniewska, International Institute of Molecular and Cell Biology in Warsaw, Laboratory of Neurodegeneration 
Summer Student 'Mutational Surface Engineering of the Restriction Enzymes and Microcalorimetric Analysis of NlaIV - dsDNA Interaction', supervised by Prof. Janusz Bujnicki, International Institute of Molecular and Cell Biology in Warsaw, Laboratory of Bioinformatics and Protein Engineering

\section{Scientific Meetings}

2015 DFG Priority Program SPP 1365-2

2014 Society for Neuroscience, Washington DC, USA

\section{Teaching Experience}

2015 Methods Course 'In vivo gene induction in cortical neurons by in utero electroporation', Göttingen Graduate School for Neurosciences, Biophysics and Molecular Biosciences

2015 Lab Rotation Supervisor, Ms Vindhya Pillai, IMPRS Neuroscience

2014 Tutor for the lecture 'Synapses, Synaptic Transmission and Network Formation', IMPRS Neuroscience

2014 Lab Rotation Supervisor, Ms Marija Radovanovic, IMPRS Molecular Biology

2012 Tutor for the lecture Nervous System, IMPRS Molecular Biology

\section{Extra-Cullicular}

2013

Co-organizer of Neurizons 2013 conference, Max Planck Institute for Biophysical Chemistry, Göttingen

2012 PhD Students Representative, Max Planck Institute of Experimental 